\title{
WASTE ISOLATION PILOT PLANT
}

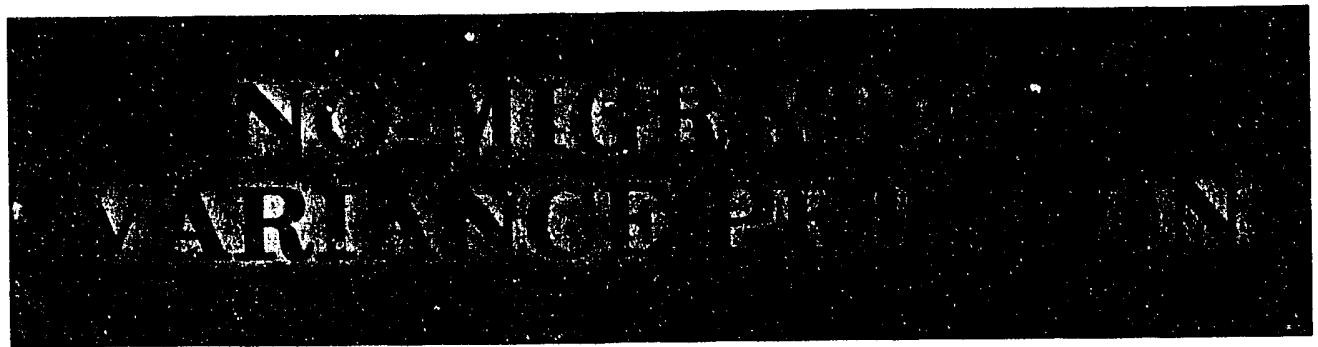

\section{FIGURES}

\author{
VOLUME VIII
}

MARCH 1990

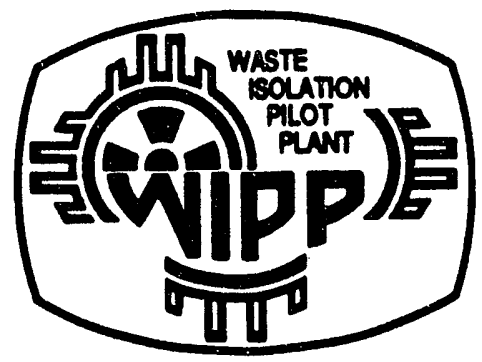

Prepared for the

Department of Energy

Under Contract DE-AC04-86AL31950

Westinghouse Electric Corporation

Waste Isolation Division

Carlsbad, New Mexico 


\section{Figure 1.4-1 Principal Activities and Interrelationships in the Seal System Development Program}

\section{DISCLAIMER}

This report was prepared as an account of work sponsored by an agency of the United States Government. Neither the United States Government nor any agency thereof, nor any of their employees, makes any warranty, express or implied, or assumes any legal liability or responsibility for the accuracy, completeness, or usefulness of any information, apparatus, product, or process disclosed, or represents that its use would not infringe privately owned rights. Reference herein to any specific commercial product, process, or service by trade name, trademark, manufacturer, or otherwise does not necessarily constitute or imply its endorsement, recommendation, or favoring by the United States Government or any agency thereof. The views and opinions of authors expressed herein do not necessarily state or reflect those of the United States Government os any agency thereof. 
DESIGN

ANALYSIS

LABORATORY TESTING

IN SITU

TESTING
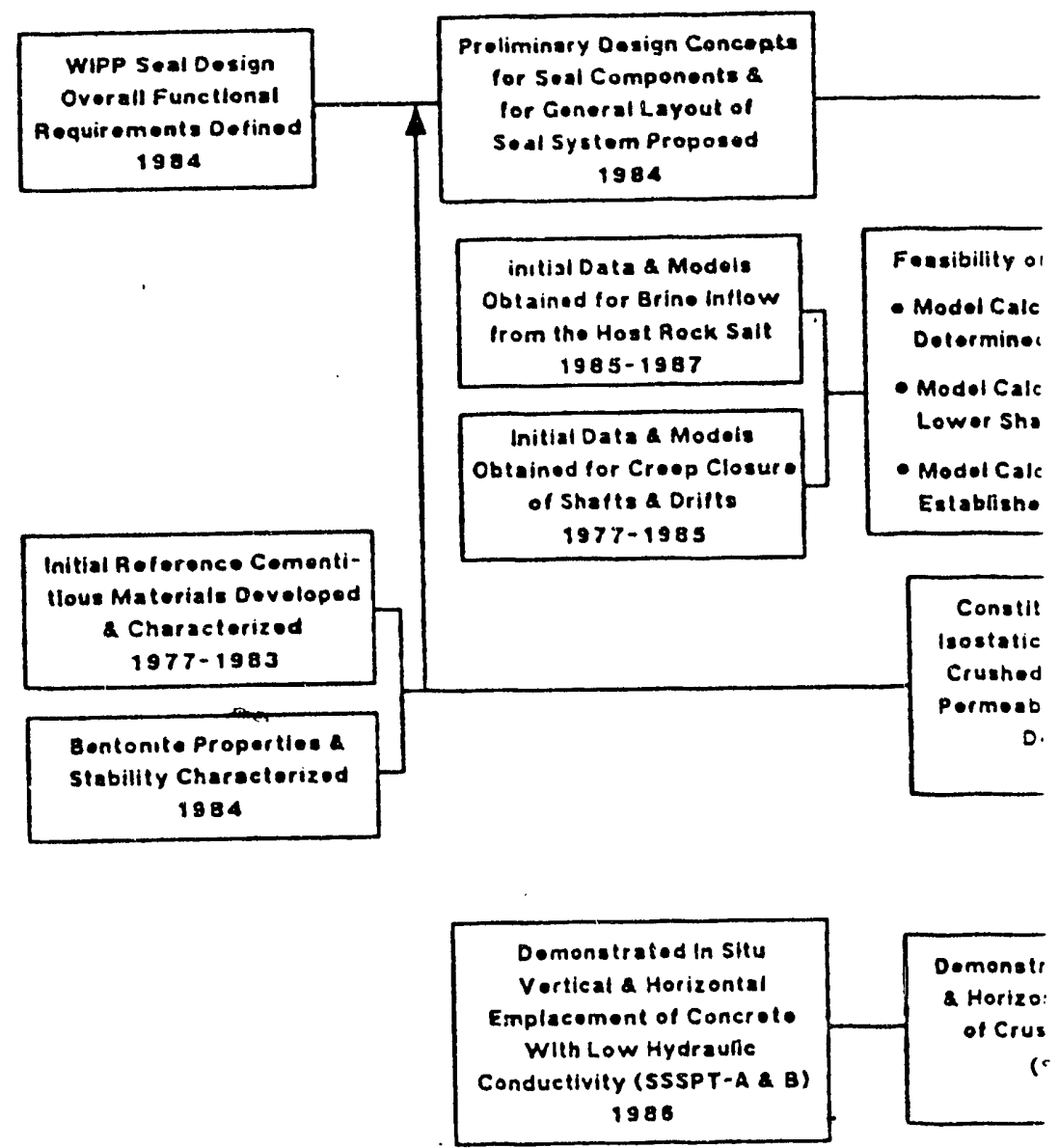

In Situ Measuremente of the

Disturbed Rock Zone (ORZ)

Near WIPP Excavations

Planned a Initialed

1984

Figure 1.4-1 Principal Activities and Interrelationships in the Seal System Develo! 


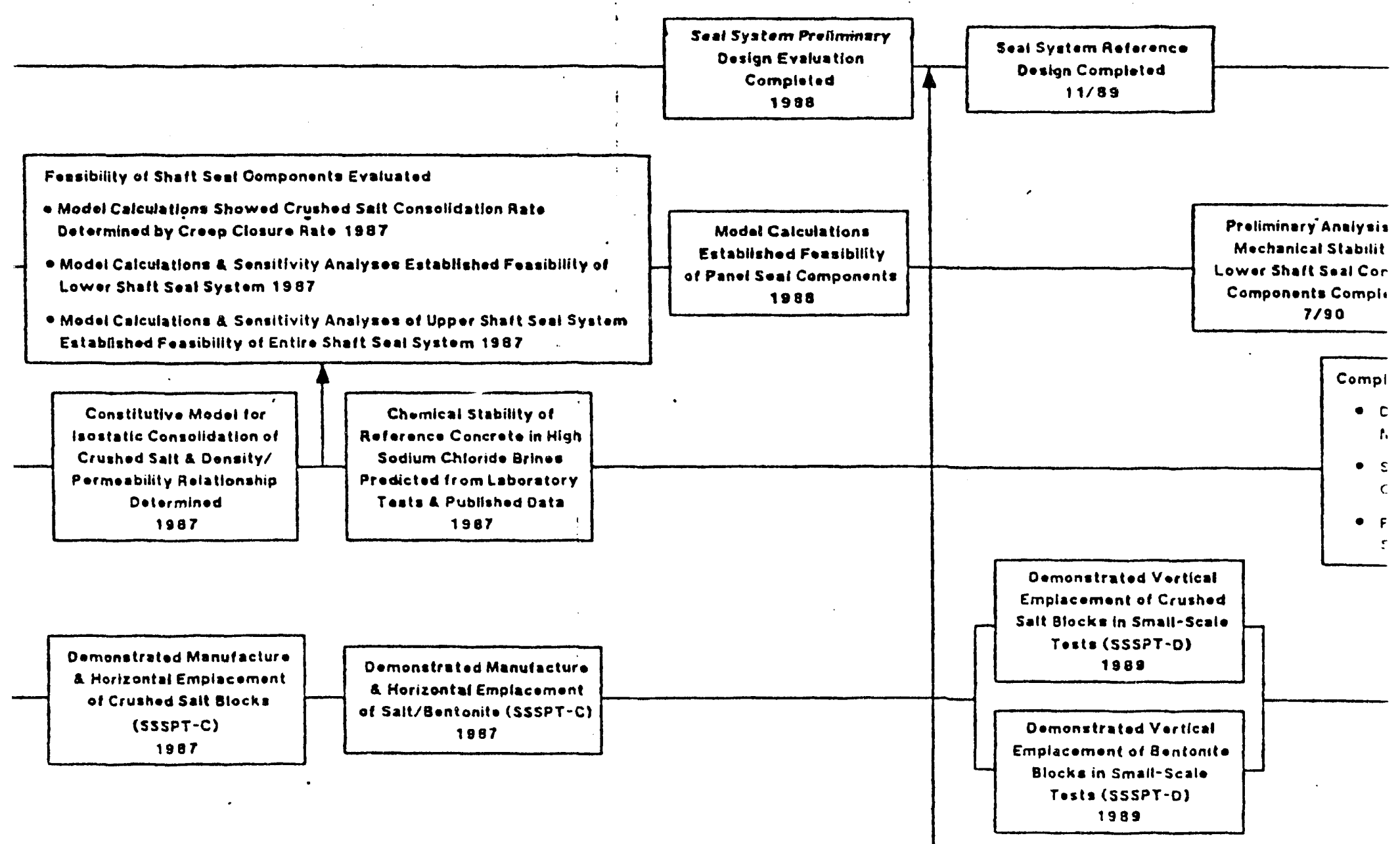

-

Interim Results irom Disturbed Rock Zone (ORZ) Measuremente Reported 1988
Interim Characteriza Interbeds Near Sole

Exeavations at the

Horizon Completec Preliminary Inlorbed G Seallng Requir eme Proposed $10 / 90$ 


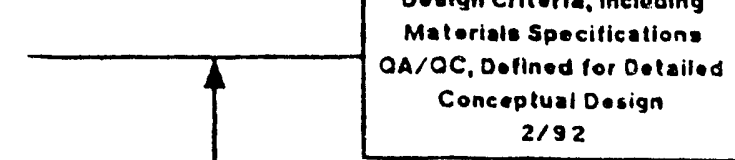

2/92
Soal Component Shapes

Dimensions Chosen Using Results

from Model Calculatione of

Mechanical Stabllity a

Fluid Flow Propertlos $7 / 92$
Detailed Conceptual Design

of Soul system Completed

$5 / 93$

\section{Seal System Performance}

Model Comploted

$7 / 92$ of S1 a Componente Prodicted

by Model Caiculations

$5 / 92$

- Grout, Bentonite \& Concrote Compatibility 3/

Obtain I Yoar Datafrom

Largo-Scalo Soal Tosls

$6 / 92$
Obtain 2 Yoar Data From

Large-scale Soal Tests $6 / 93$
Oblain J Year Dala From

Large-Scale Seal Teste $6 / 94$

al 


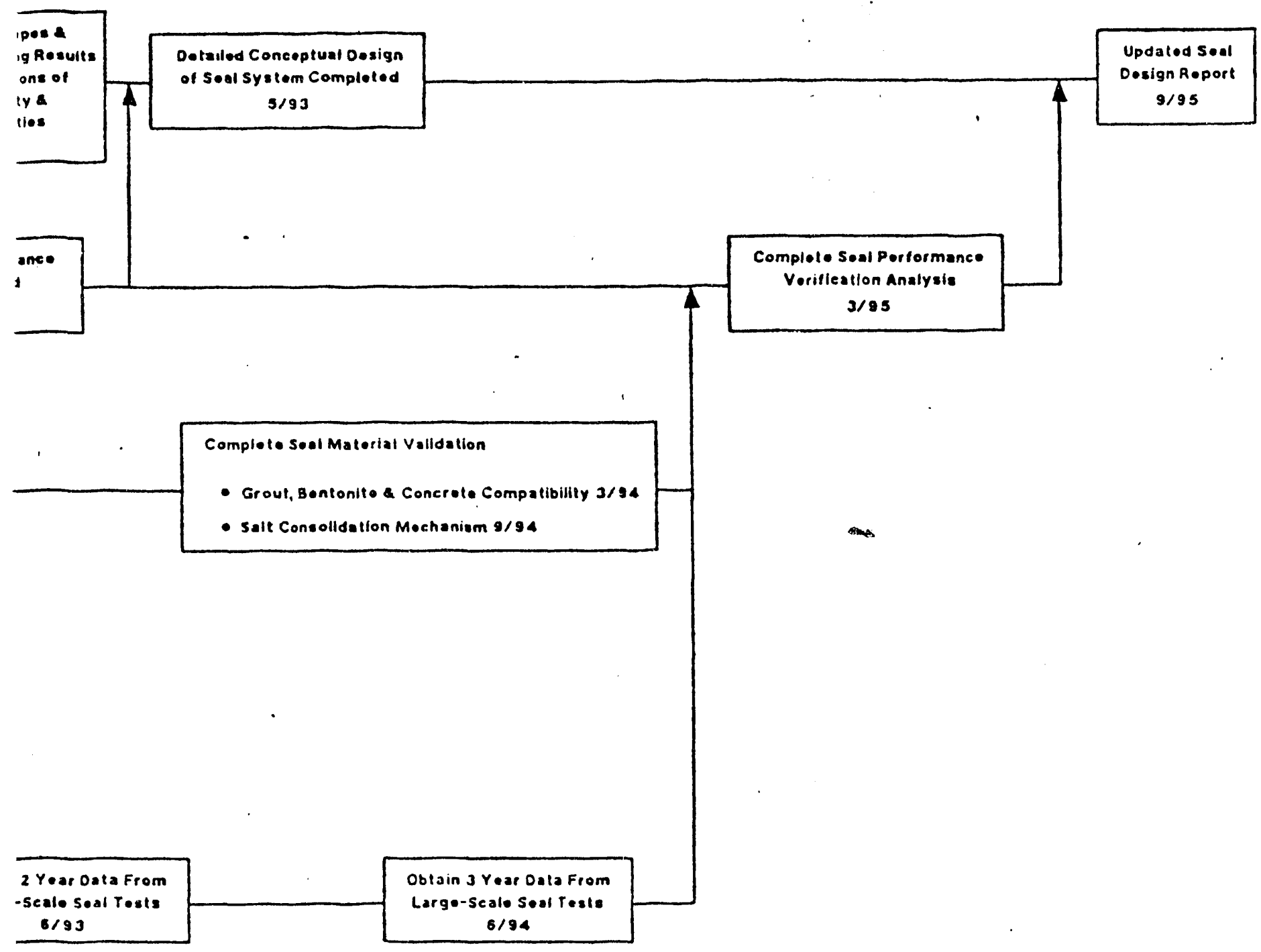




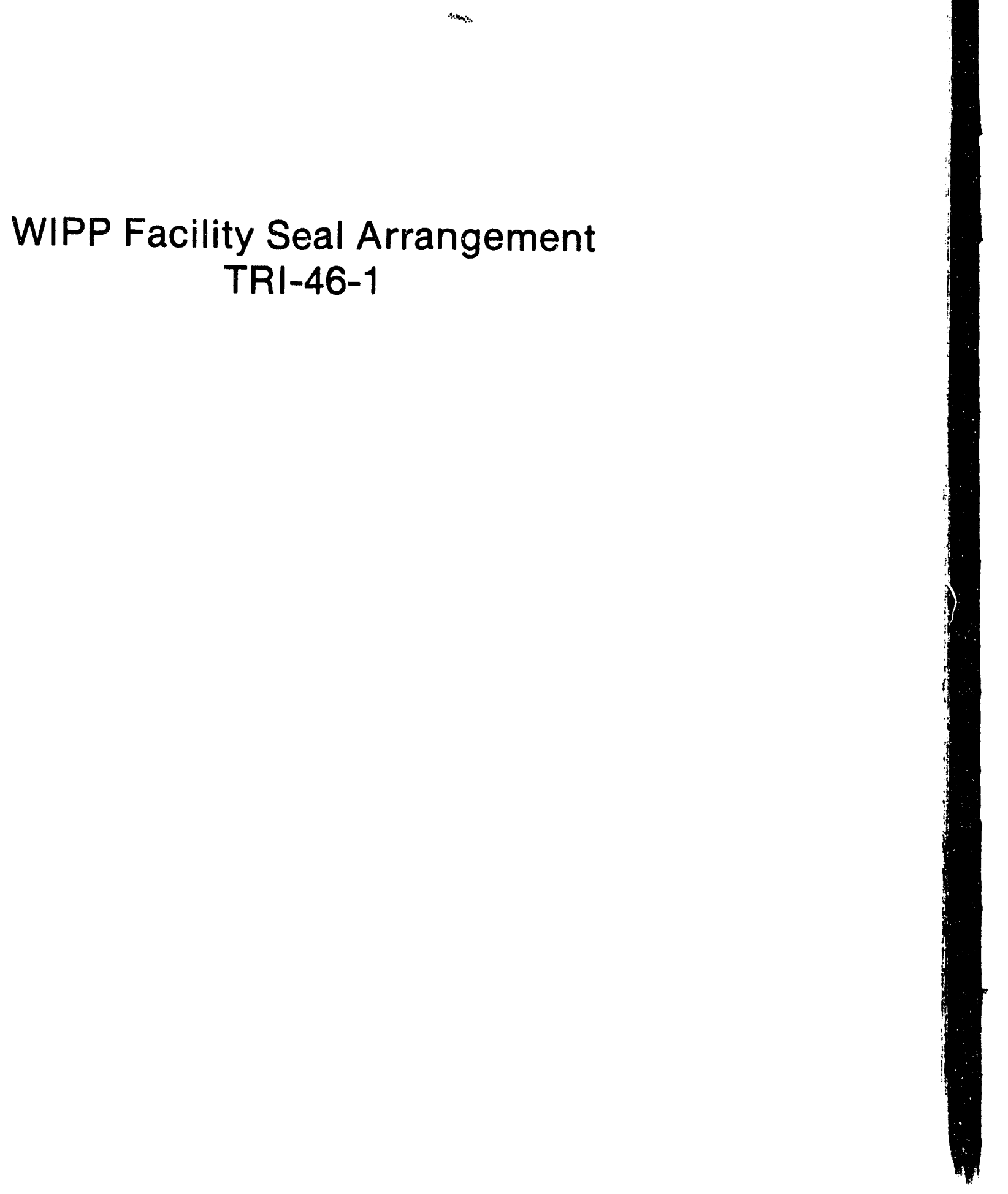



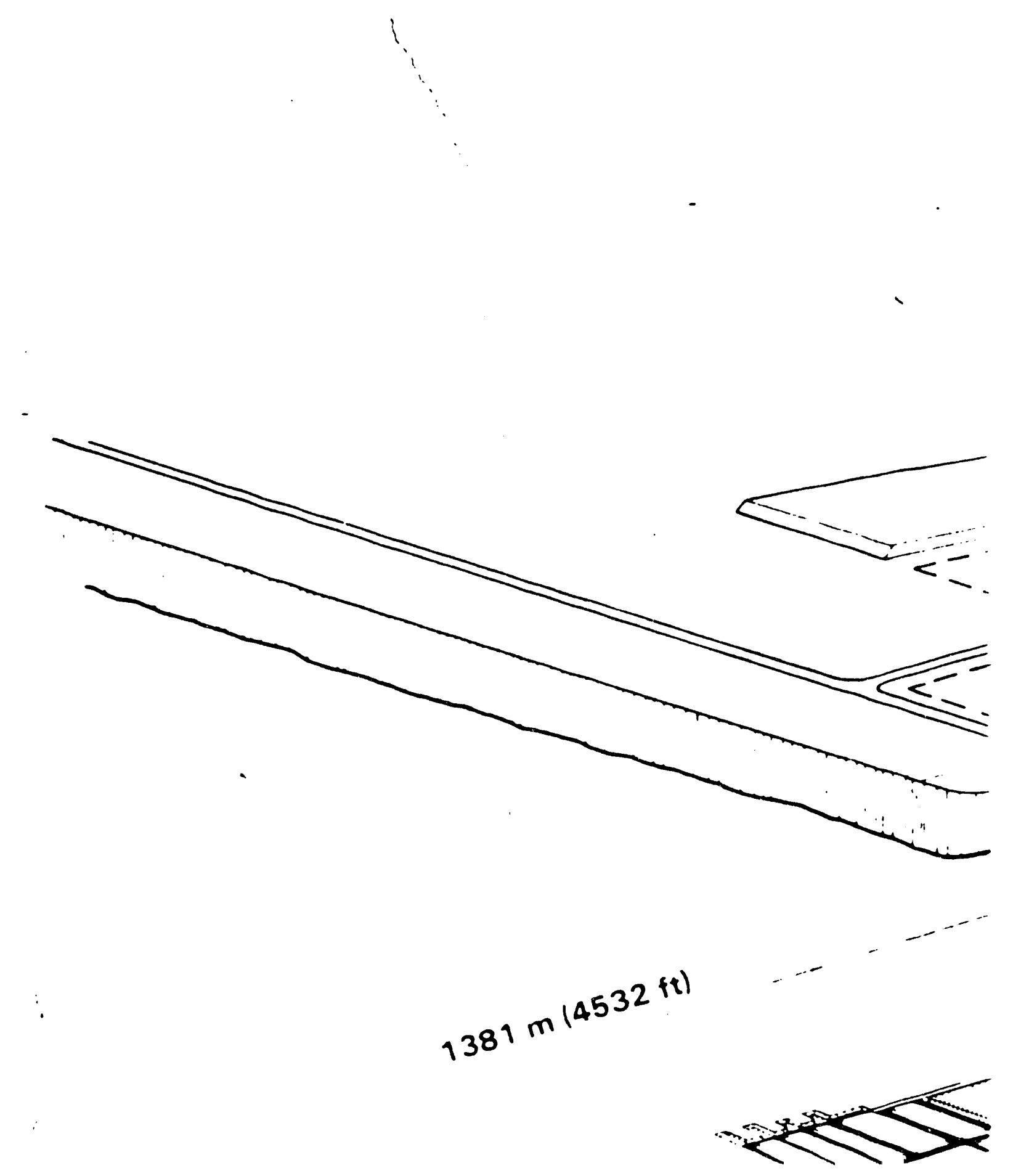

$1381 m\left(4.532^{\mathrm{ft})}\right.$

, 


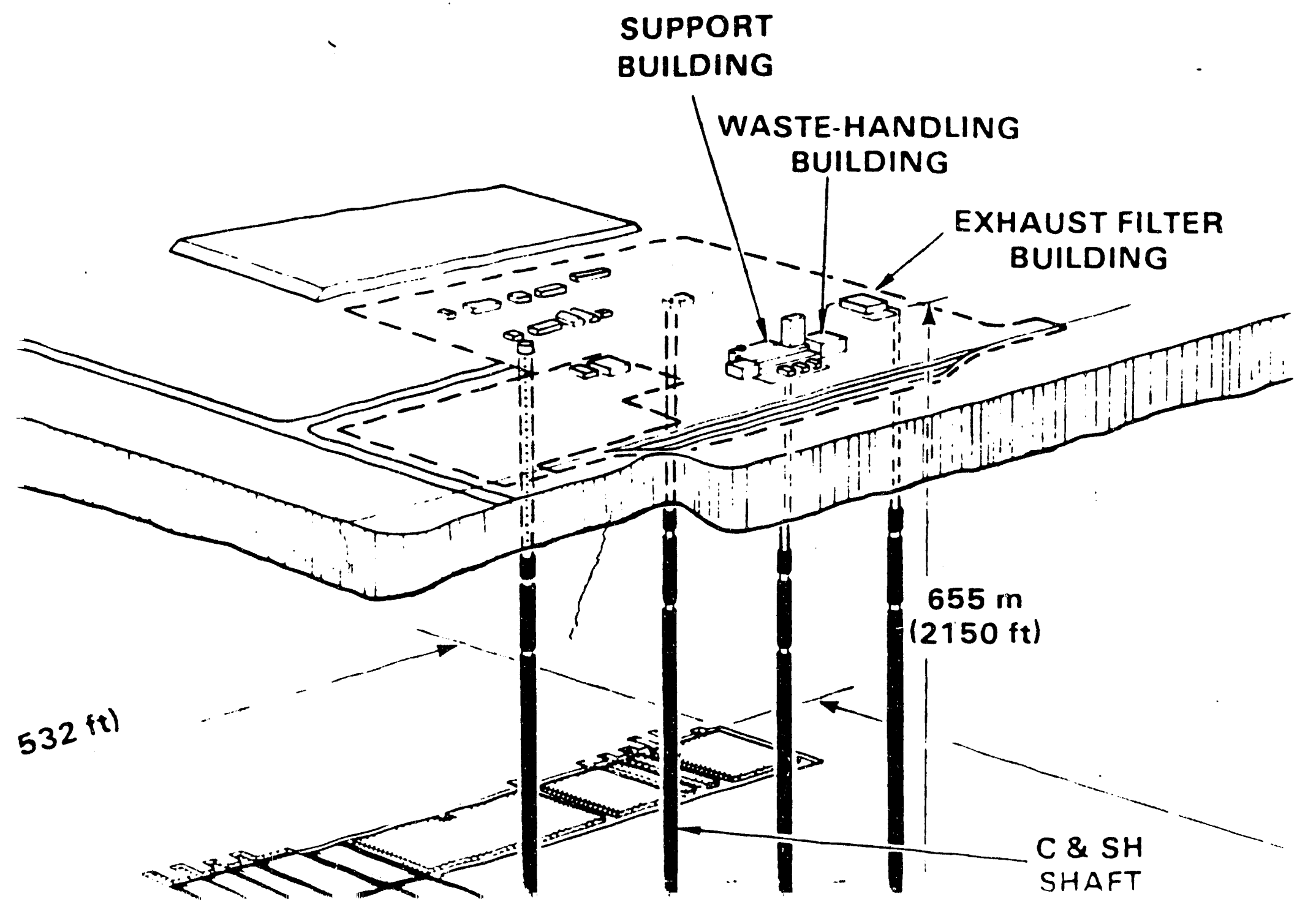




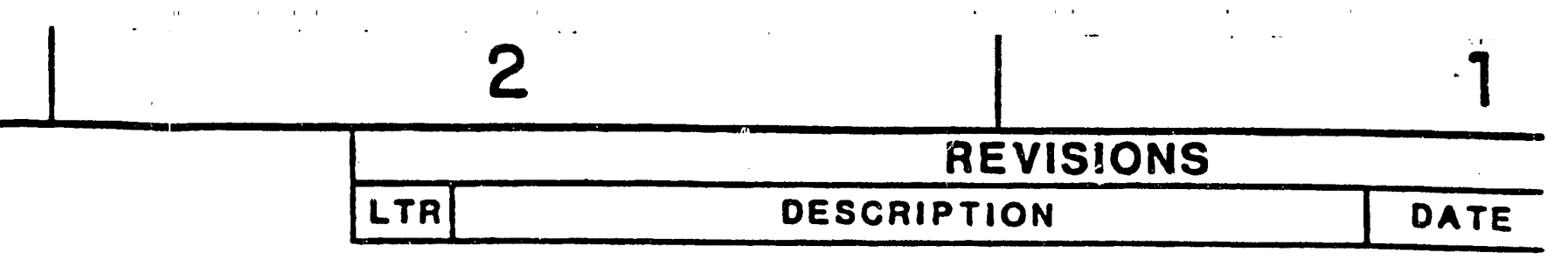

sanes 



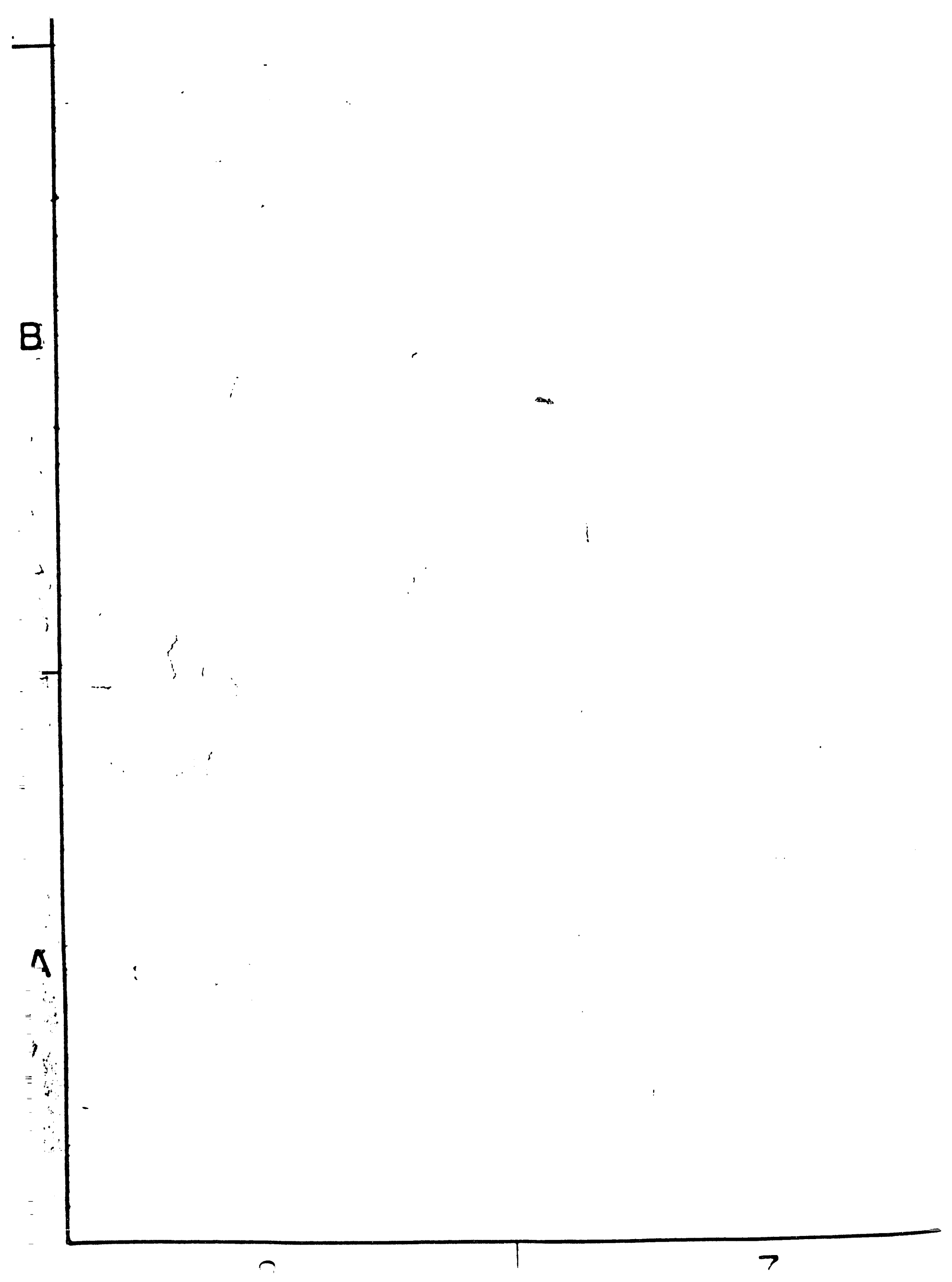




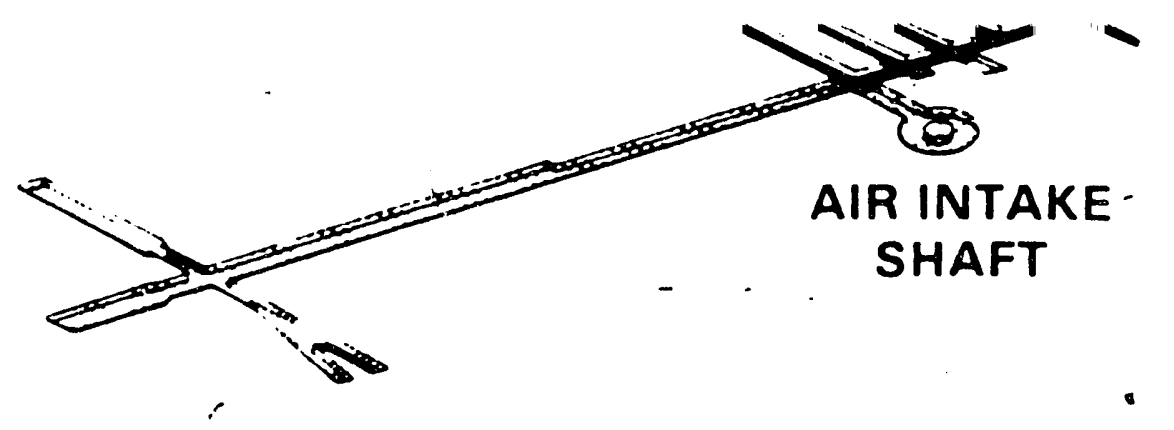

\section{LEGEND}

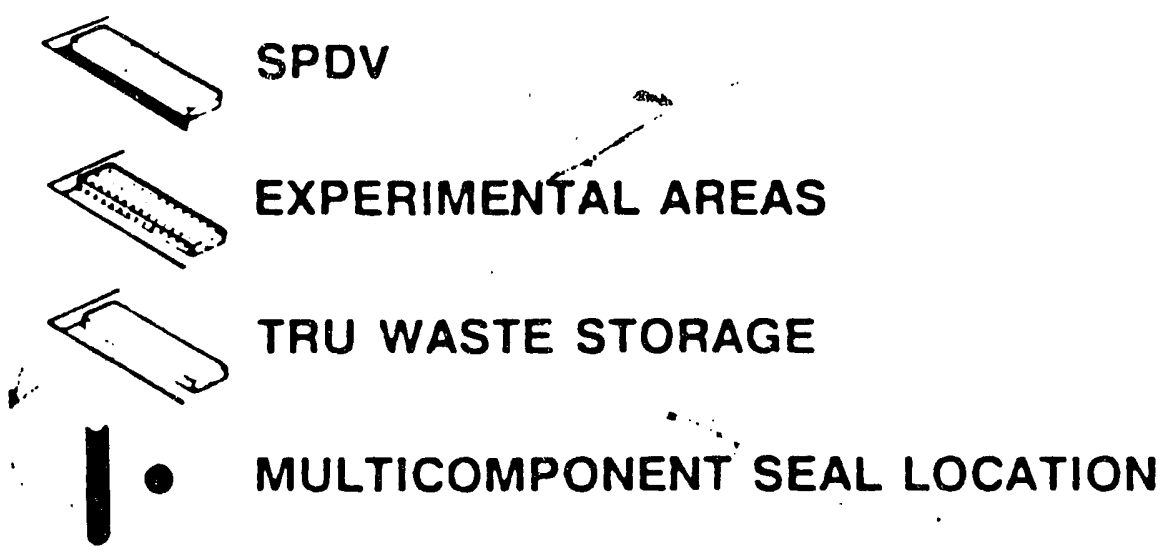

ABBREVIATIONS

SPDV - SITE \& PRELIMINARY DESIGN VALIDATION

C \& SH - CONSTRUCTION \& SALT HANDLING 


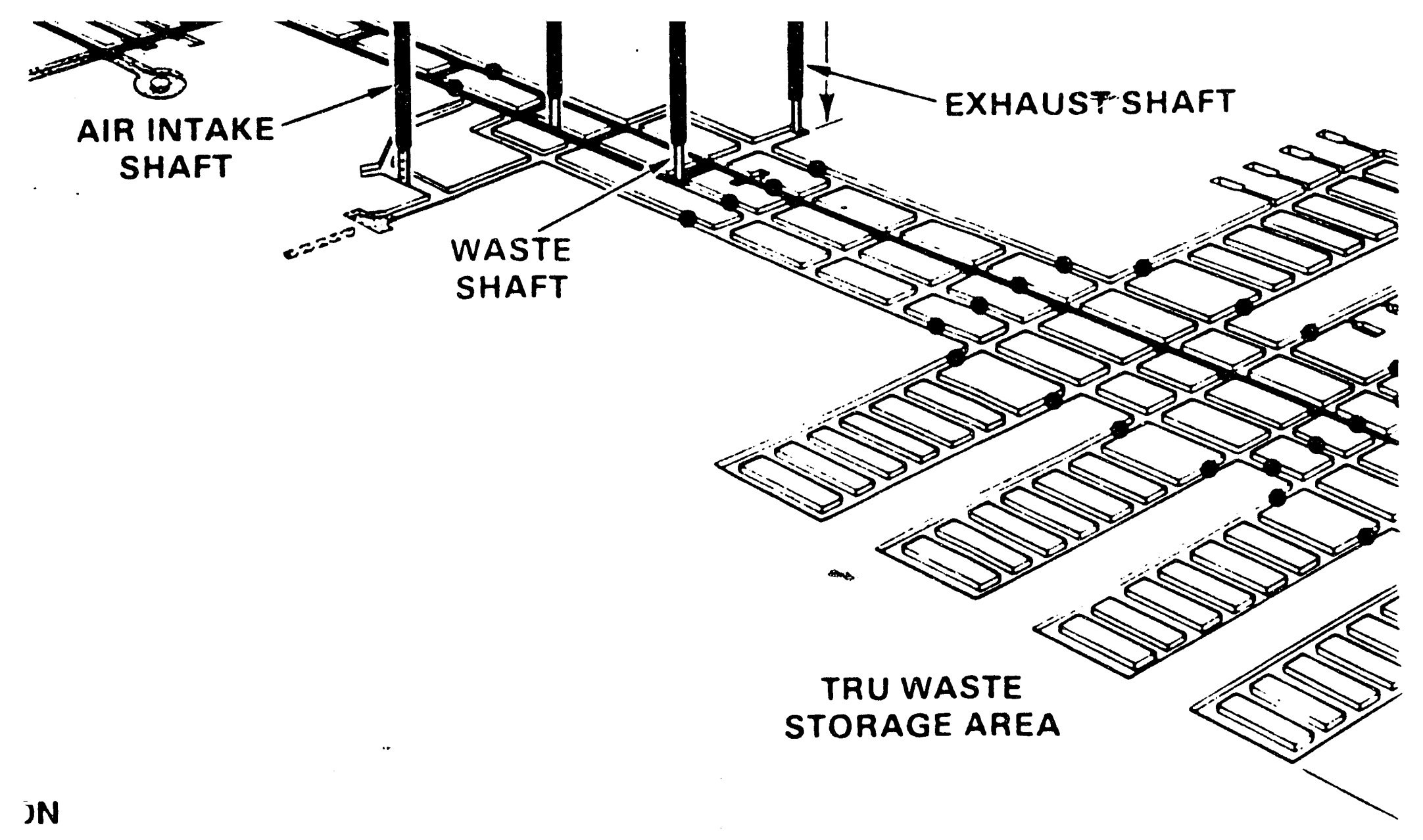




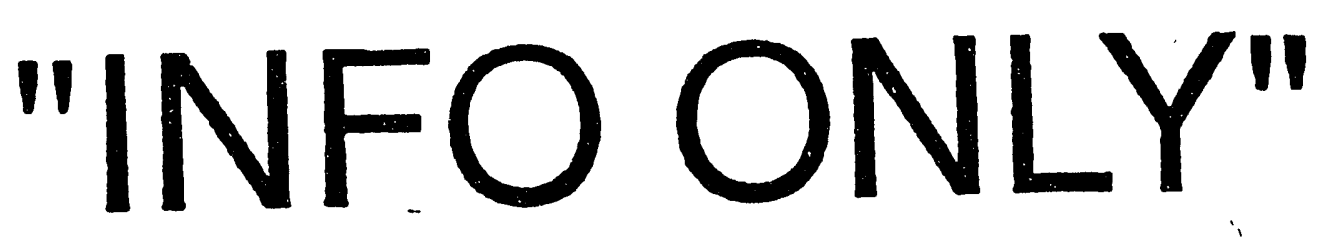

in Sandia National Laboratories

$A$

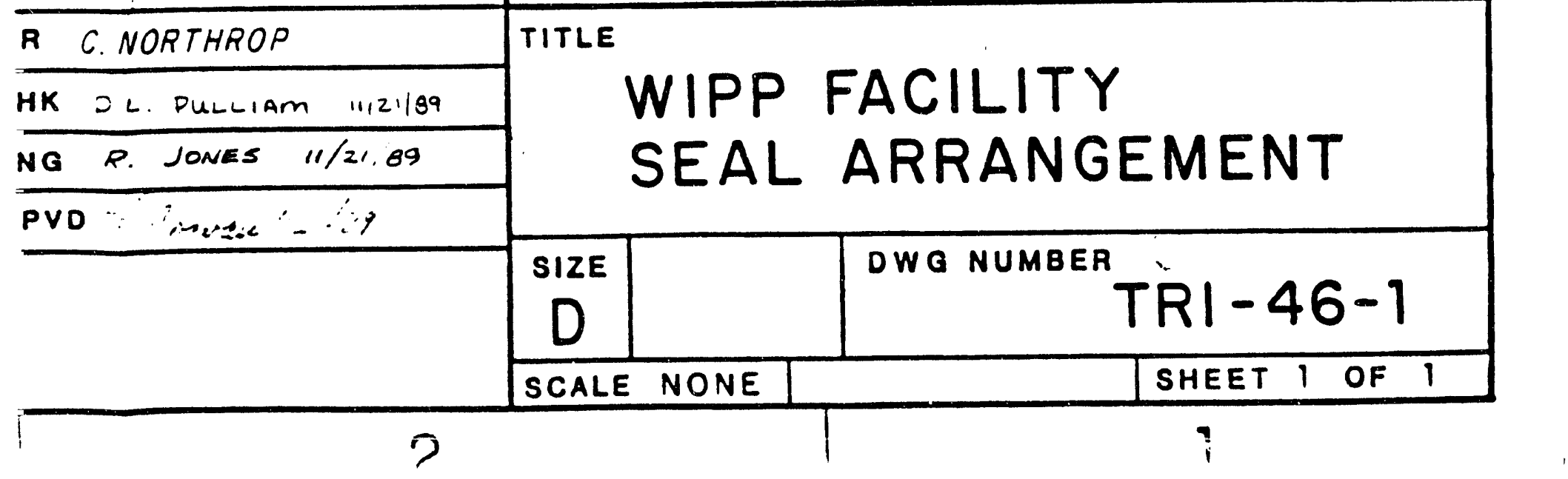


Shaft Seals General Arrangement TRI-46-2 




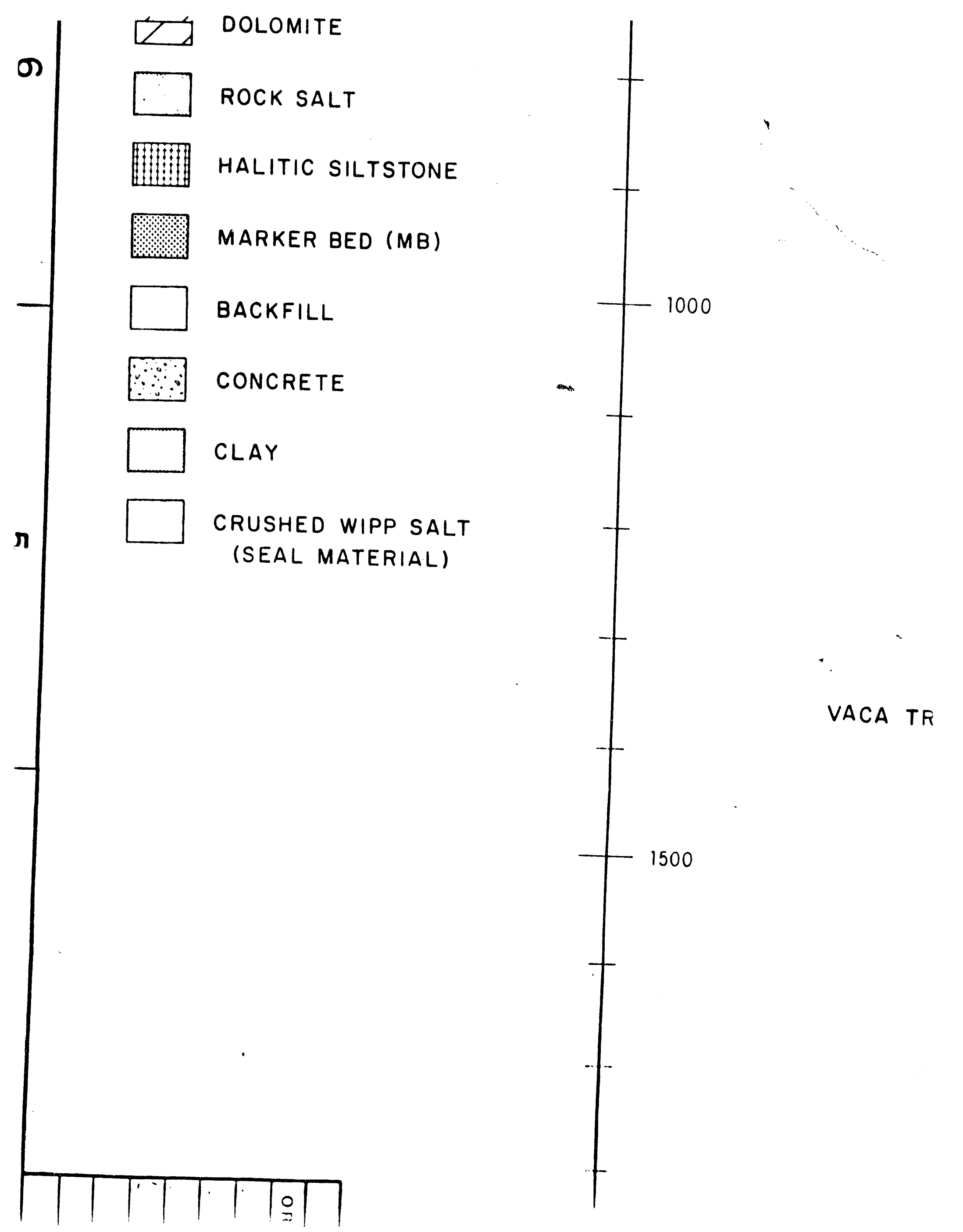




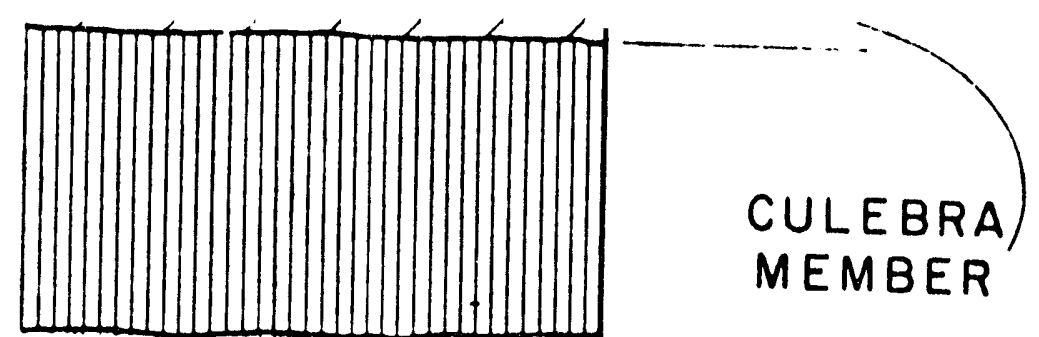

000
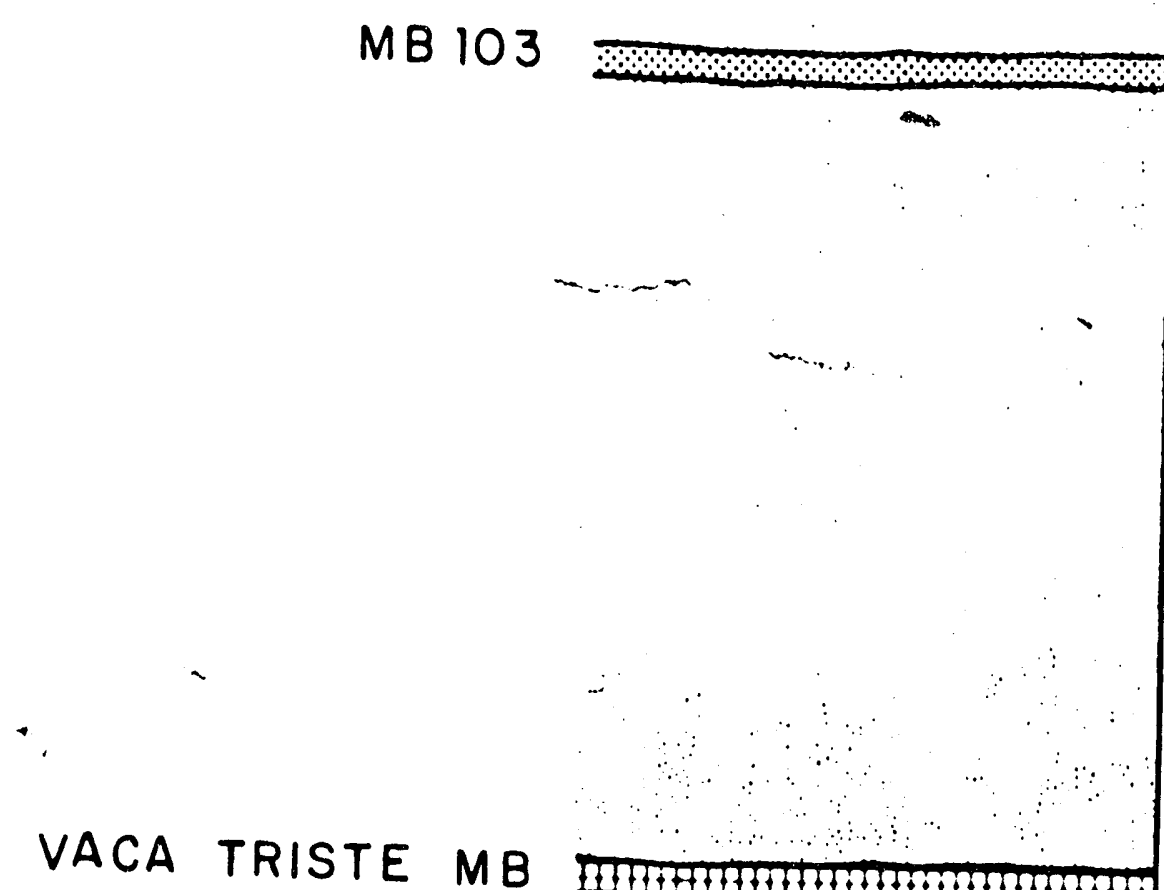

MEMBER

00

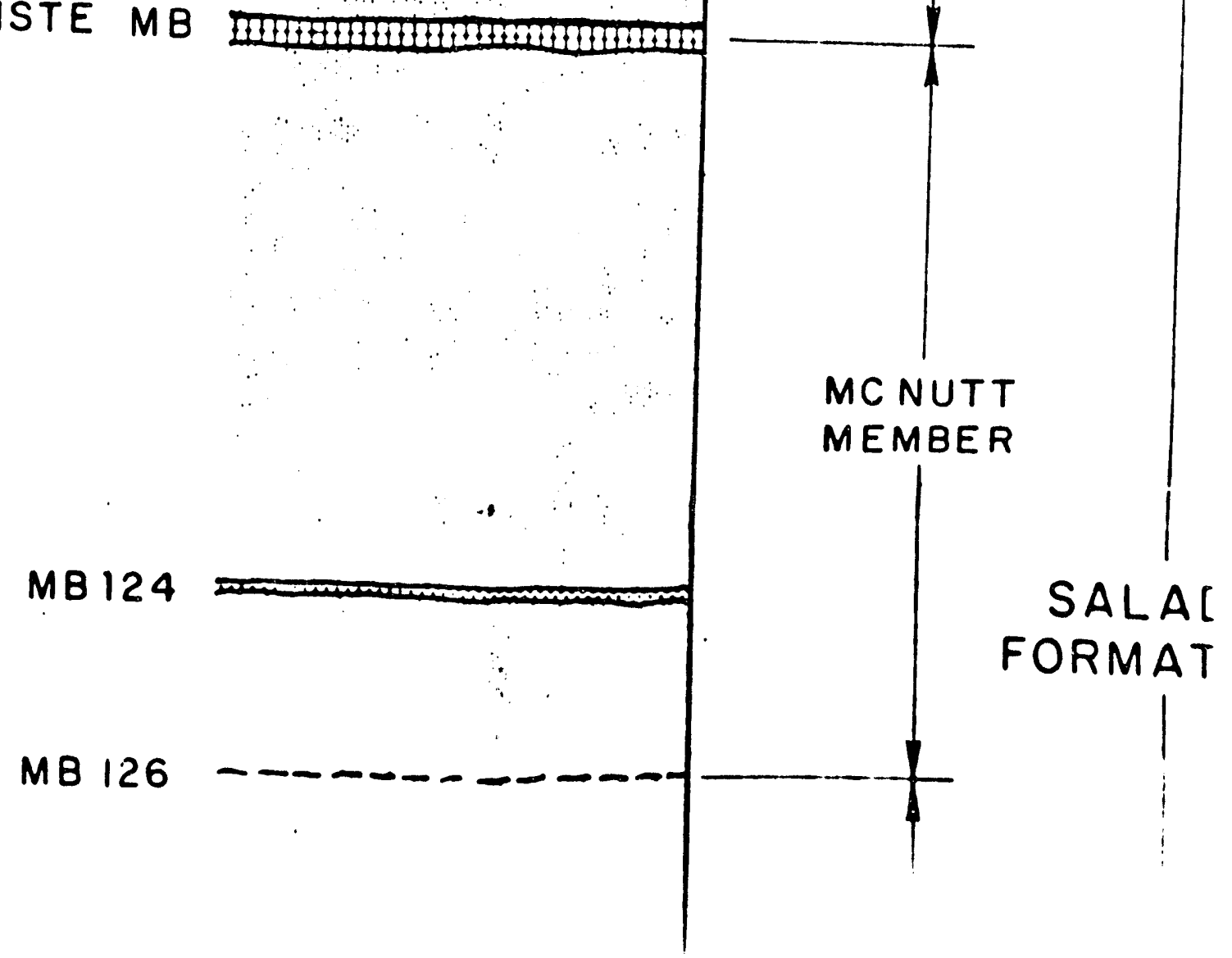




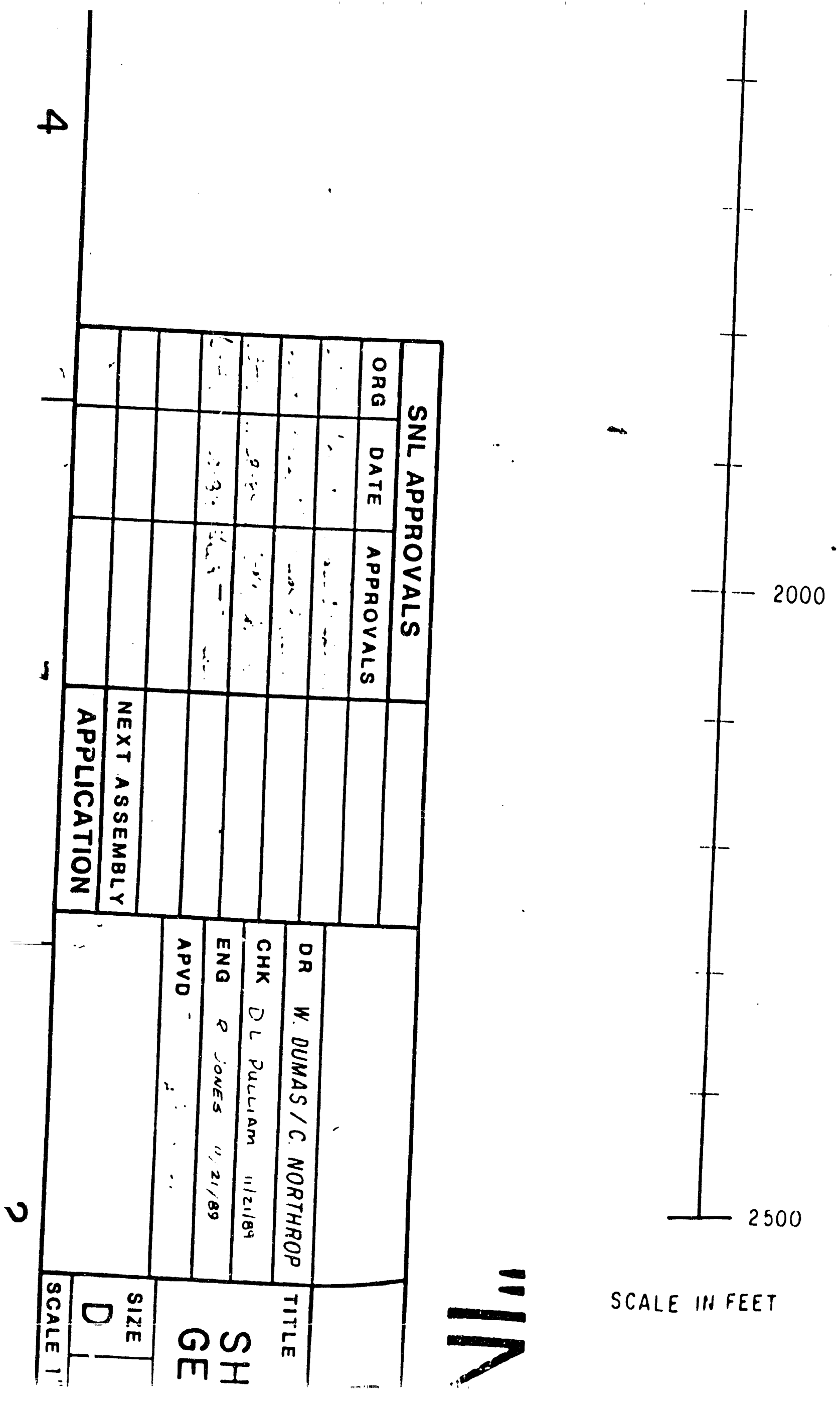




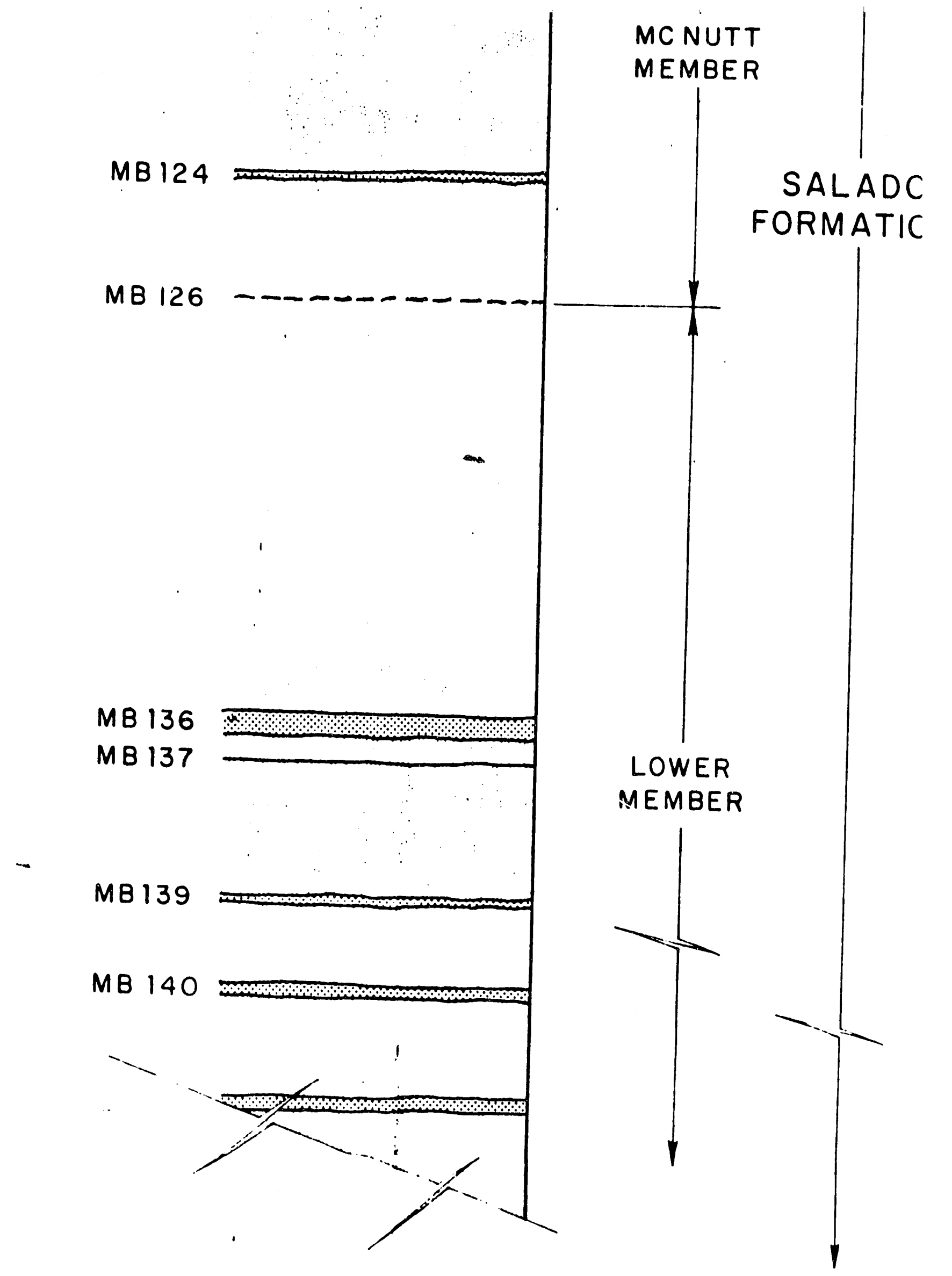




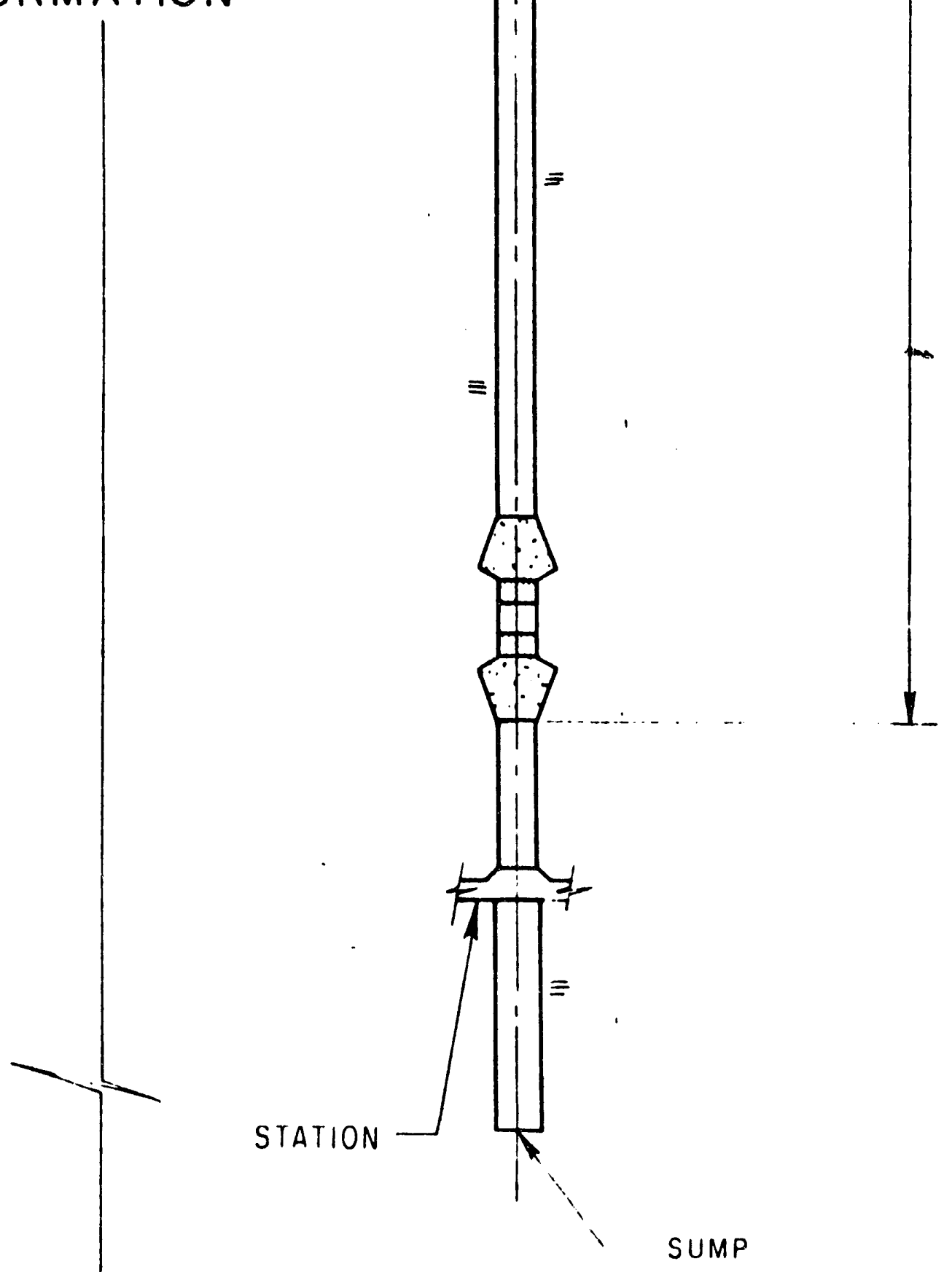




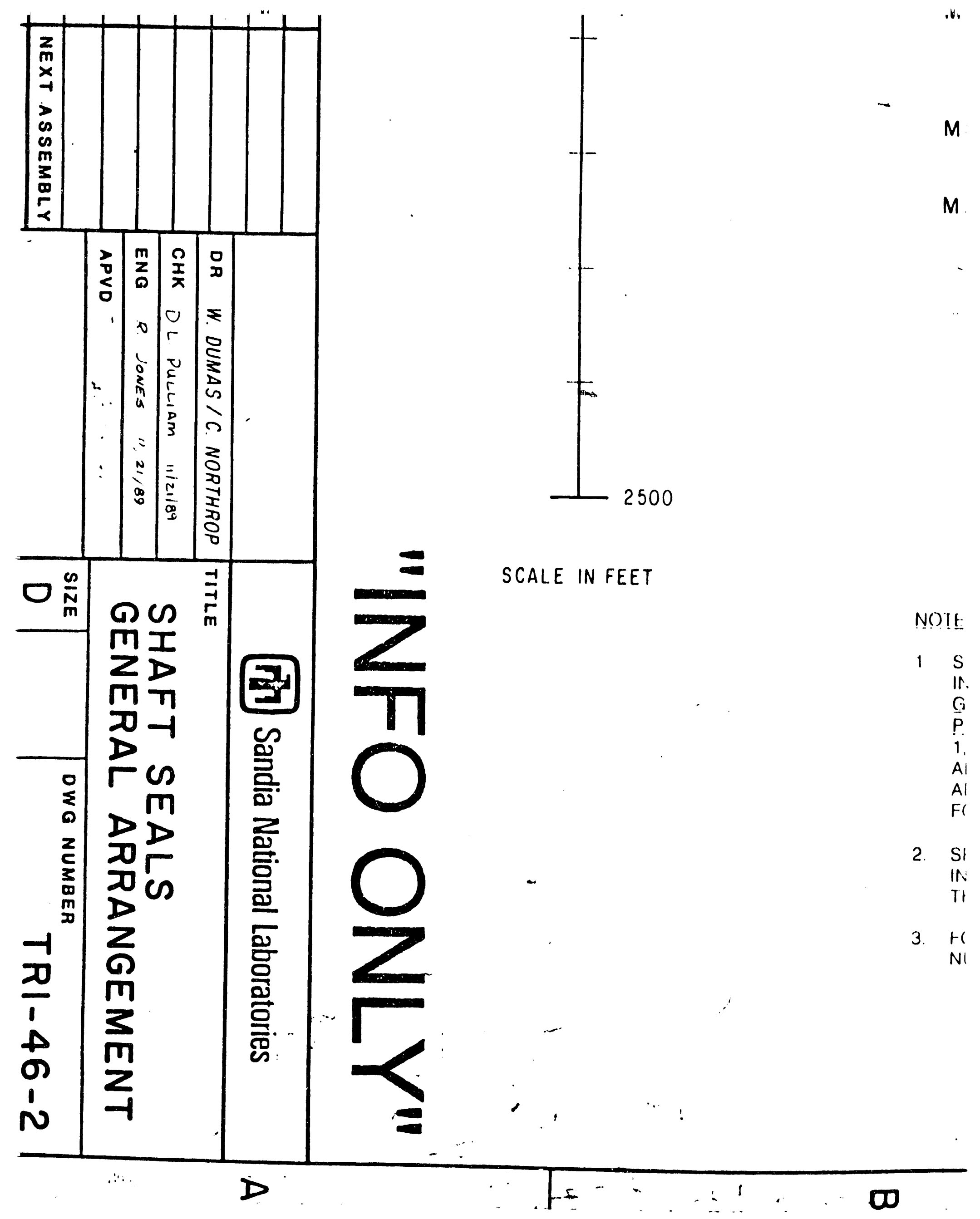




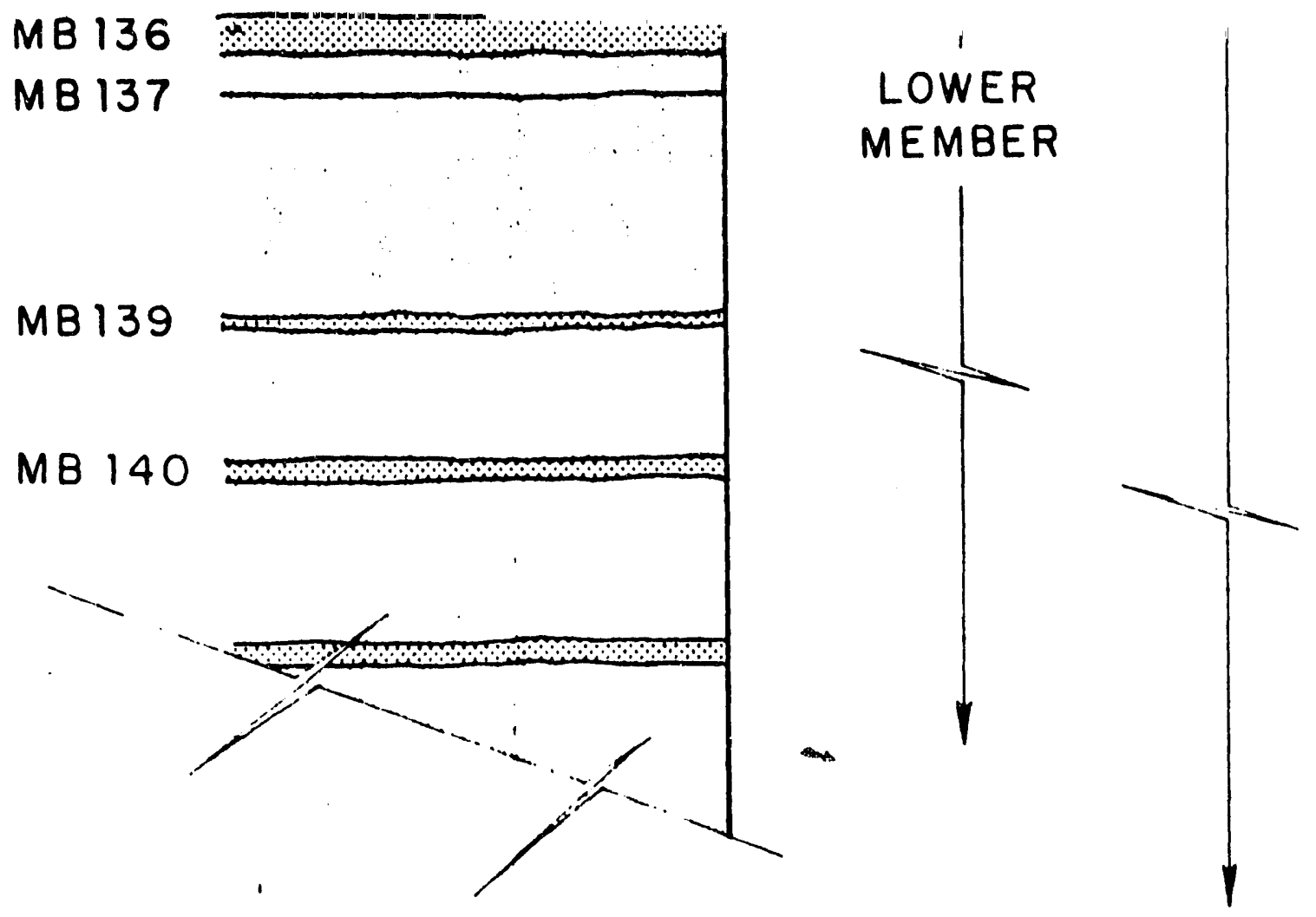

STATIC

NOIES

1 STHATIGHAPHY IS REPRESENTED ACCORDING TO INFORMATION IN D.W. POWERS ET AL., AUGUST 1978, GEOLOGICAL CHARACTERIZATION REPORT, WASTE ISOLATION PILOT PLANT (WIPP) SITE SOUTHEASTERN NEW MEXICO, VOL. 1. SAND 78-1596, SANDIA NATIONAL LABORATORIES, ALBUQUERQUE, NM. STRATIGRAPHY HAS BEEN SIMPLIFIED ABBOVE THE RUSTLER FORMATION IN THE SUPRA RUSTLER FORMATION

2. SPECII IC UIMENSIONS AND SIGNIFICANT ELEVATIONS FOR INDIVIDUJAL SHAFTS CAN GE FOUND IN DWG. TRI-46-2. SHEETS 2 THFIOUSGH 4

3. FOR A SUMMARY OF SHAFT INFORMATION SEE DRAWING NUMBER TRI.46.2, SHEET 5 OF 5. 
Shaft Seals Water Bearing Zone Plan and Section TRI-46-2 

:R

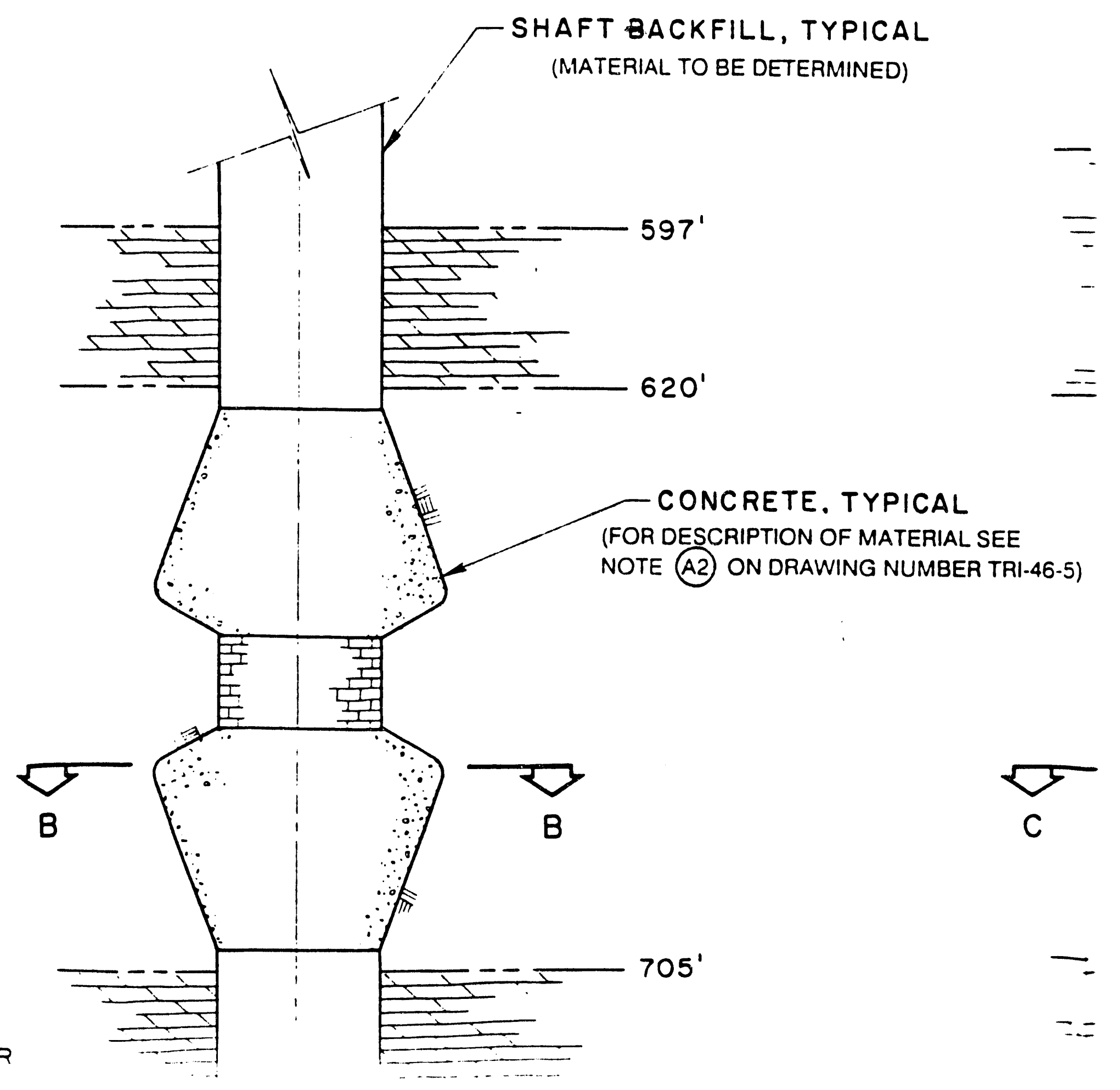




\section{MEMBER}

ERIAL SEE

UMBER TRI-46-5)
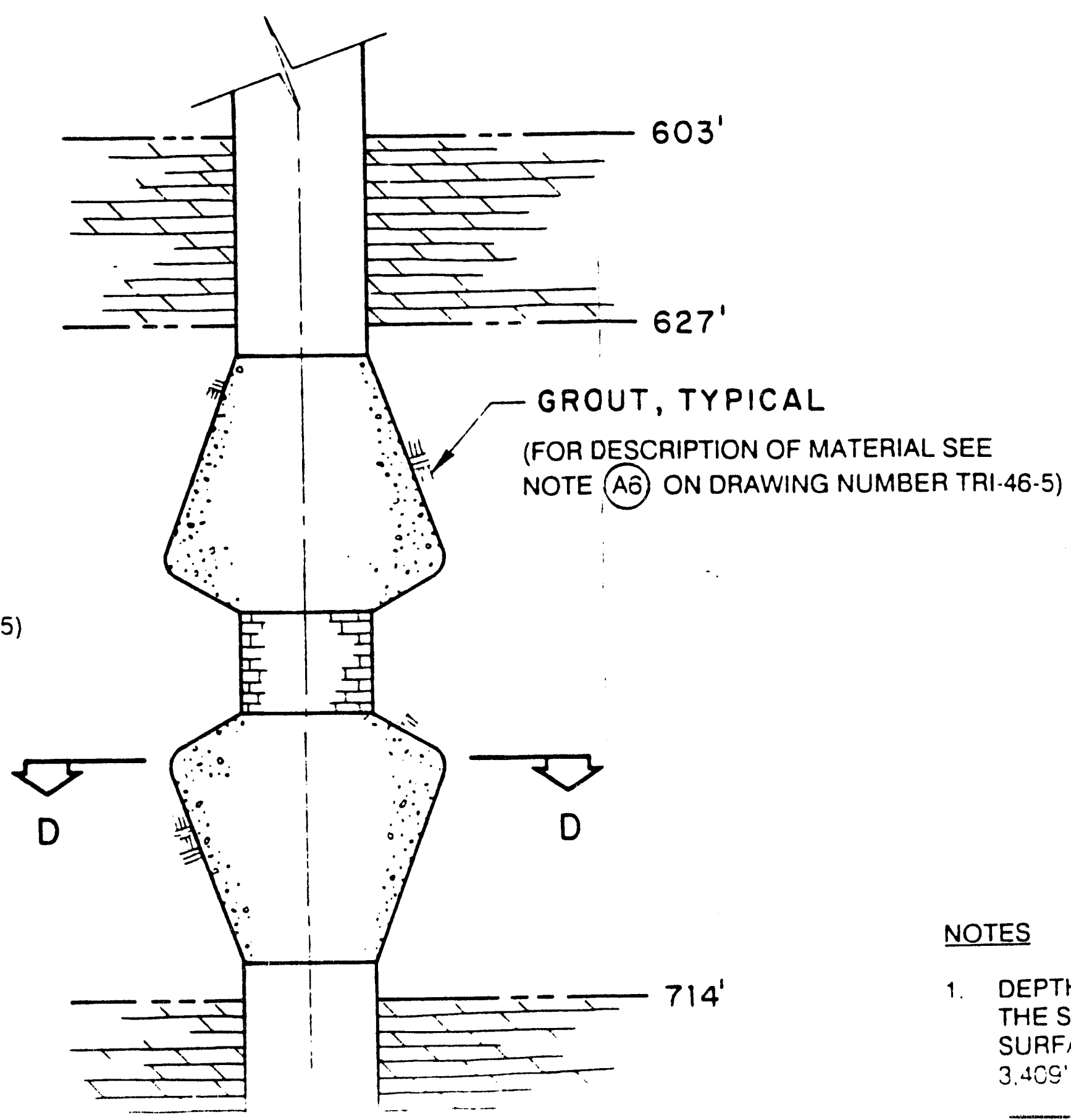

-

1. DEPTHM THE SUR! SURFACE 3. $409^{\prime} \cdot \mathrm{O}^{\circ}$ 


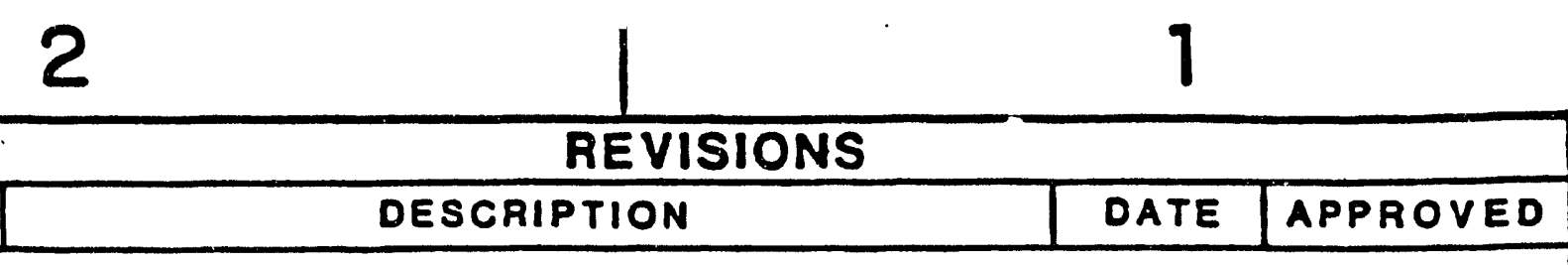

$-603^{\prime}$

$-627^{\prime}$

UT, TYPICAL

ESCRIPTION OF MATERIAL SEE

A0) ON DRAWING NUMBER TRI-46-5)

NOTES

$-714^{\prime}$

1. DEPTH MEASUREMENTS INDICATE NUMBER OF FEET BELOW.

: THE SURFACE. WHHERE THE SURFACE EQUALS 0'-0". THE

- SURFACE ELEVATION THAT IS USED AS THE REFERENCE IS "3.4OG'. 0 " (USGS :S2? NORTH AMERICAN DATUM). 


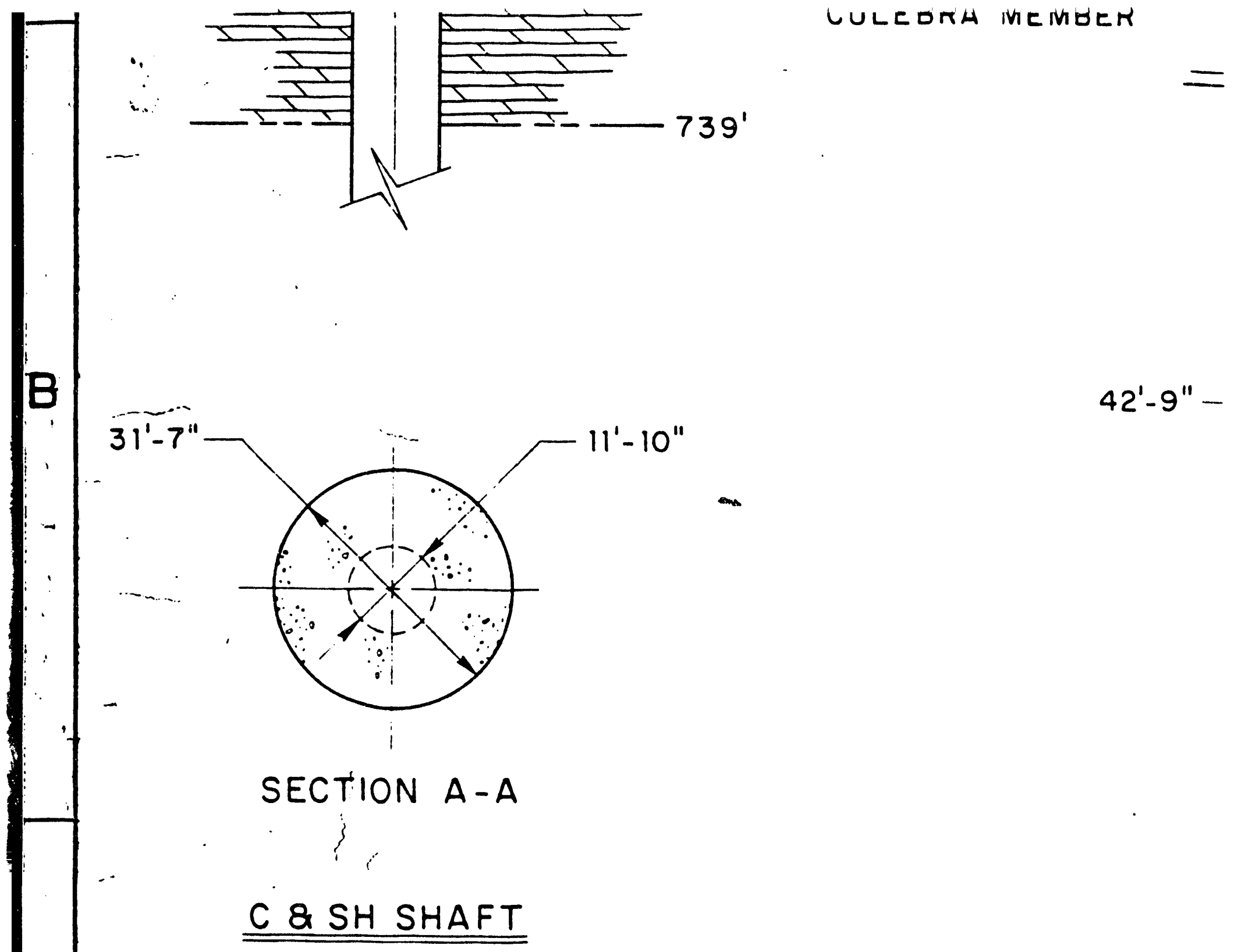

UULEONA MEMUEK

$A$

* NOTE:

THESE DIMENSIONS AND ANGLES ARE TYPICAL FOR ALL SHAFT CONFIGURATIONS. 


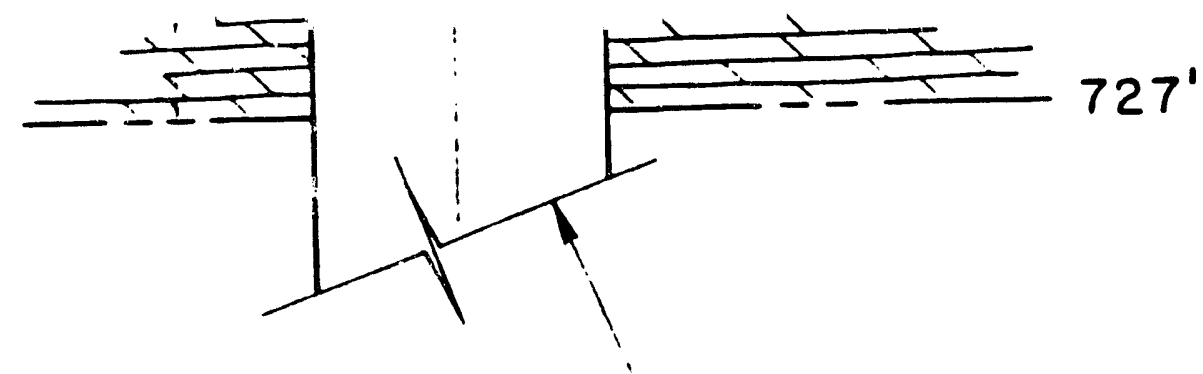

SHAFT BACKFILL, TYPICAL (MATERIAL TO BE DETERMINED)

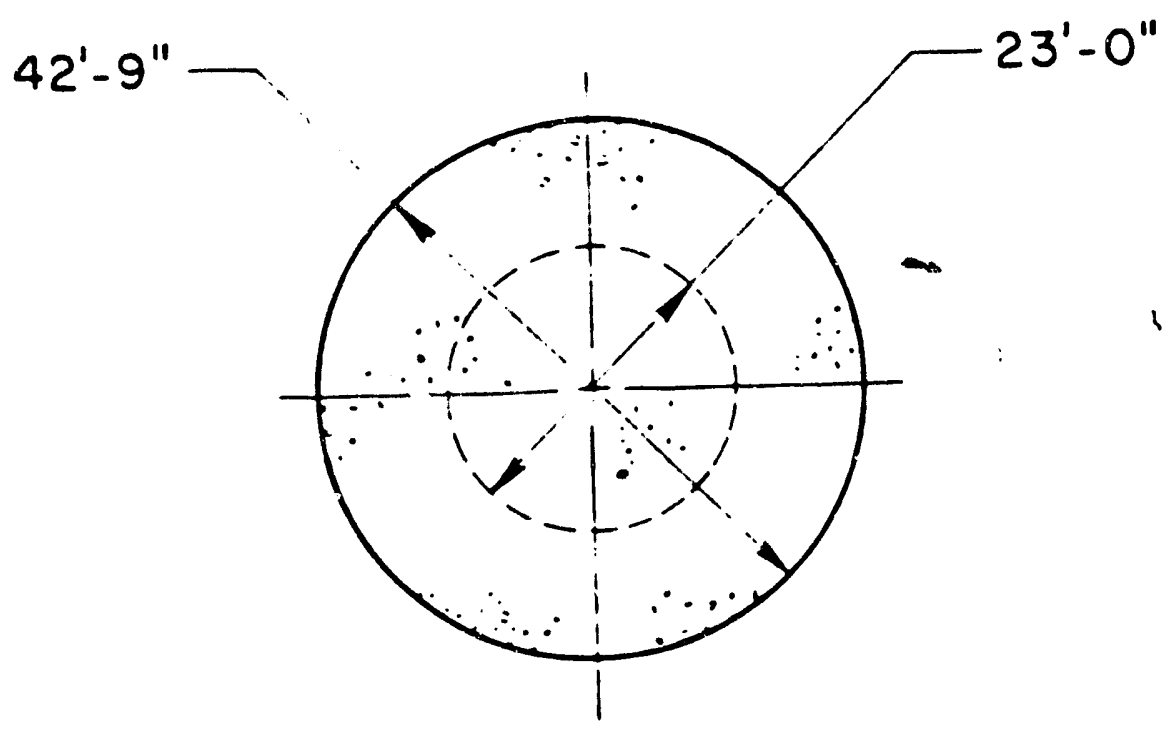

SECTION B-B

WASTE SHAFT

¿S ARE TYPICAL ONS. 


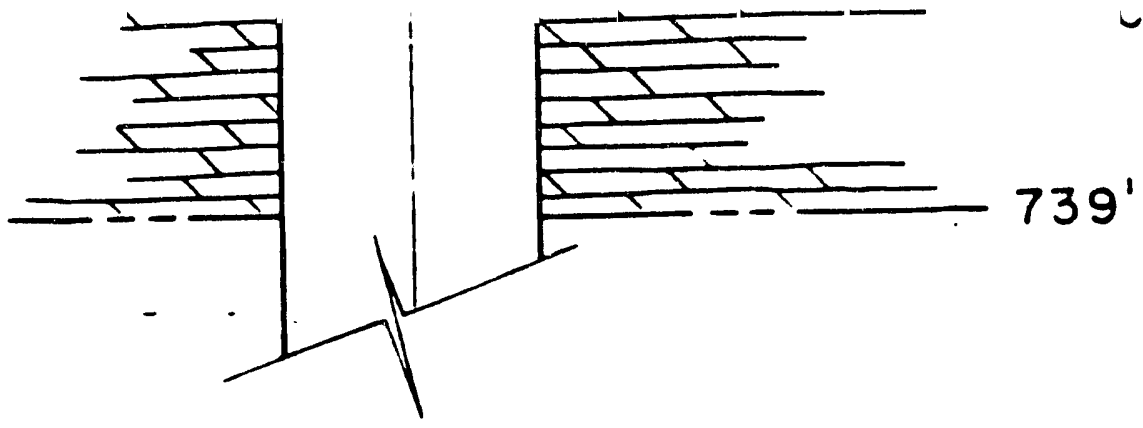

T BACKFILL, TYFICAL ERIAL TO BE DETERMINED)

$23^{\prime}-0^{\prime \prime}$

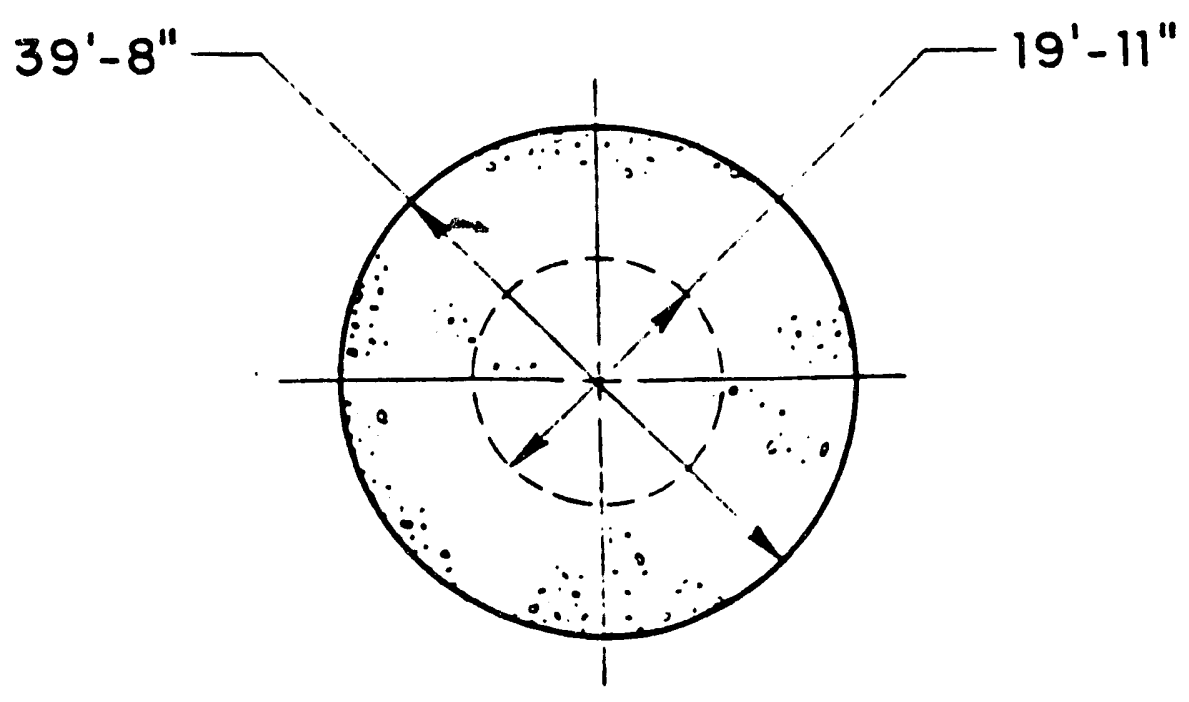

SECTION C-C

AIR INTAKE SHAFT

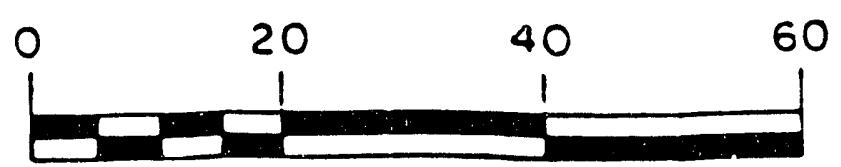

SCALE IN FEET 


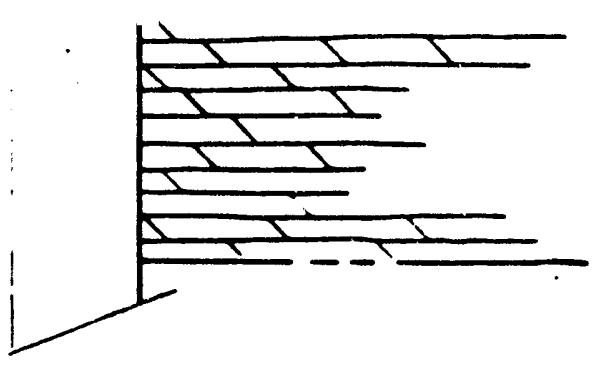

CULEBRA MEMBER
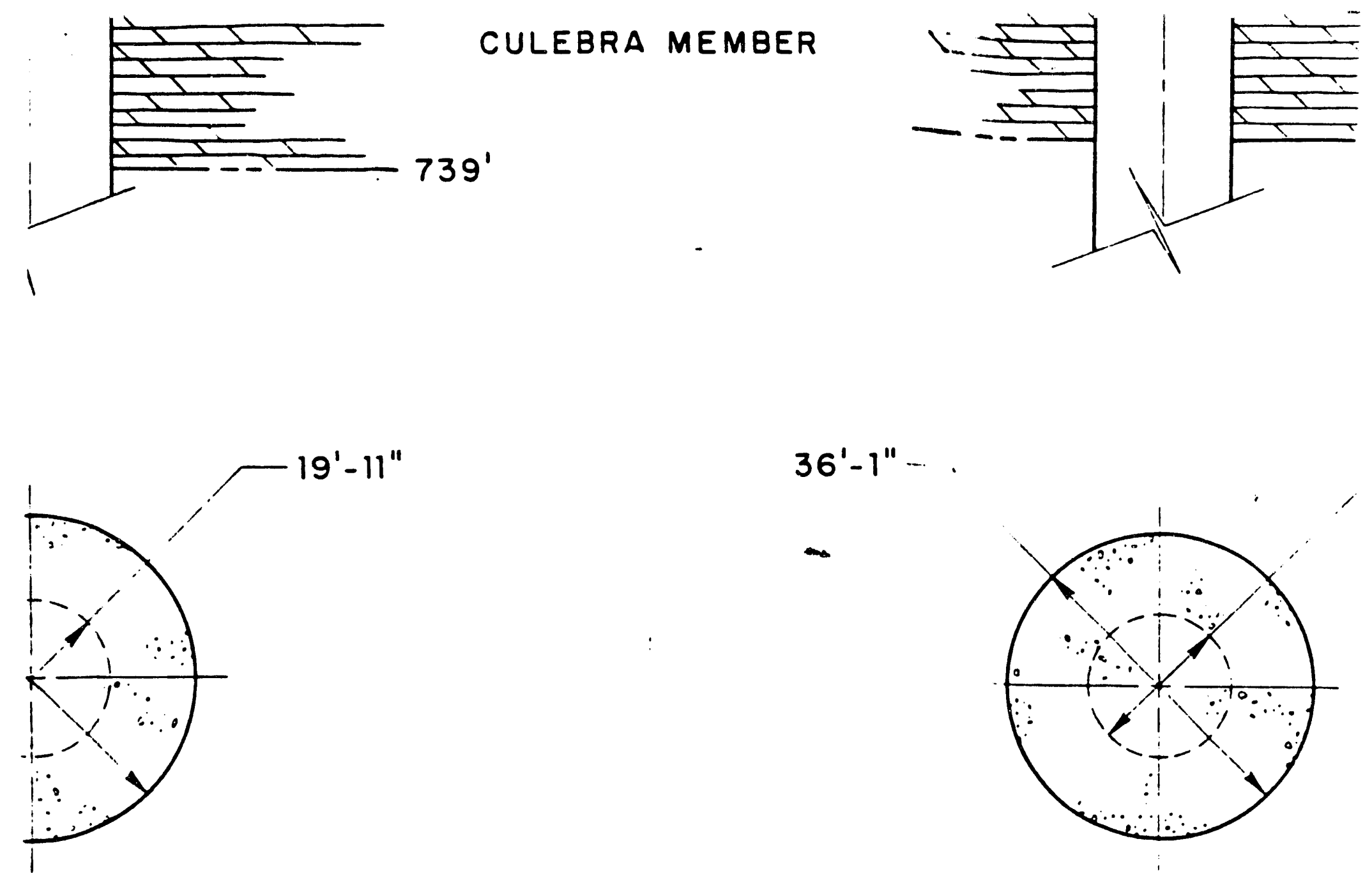

$3 \mathrm{~N} \mathrm{C}-\mathrm{C}$

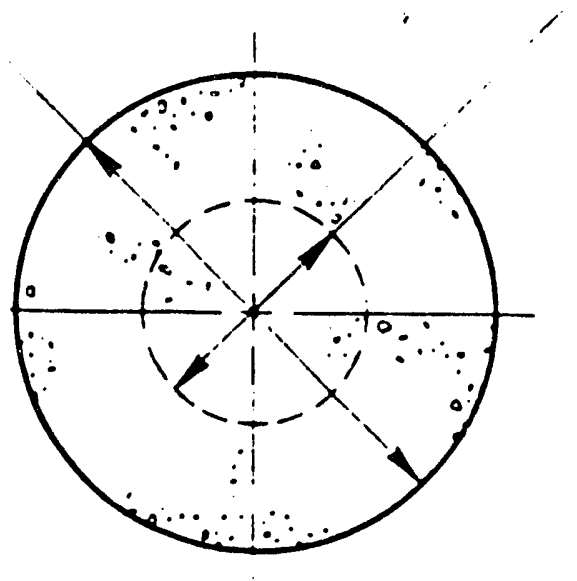

SECTION D-D

IE SHAFT

EXHAUST SHAFT

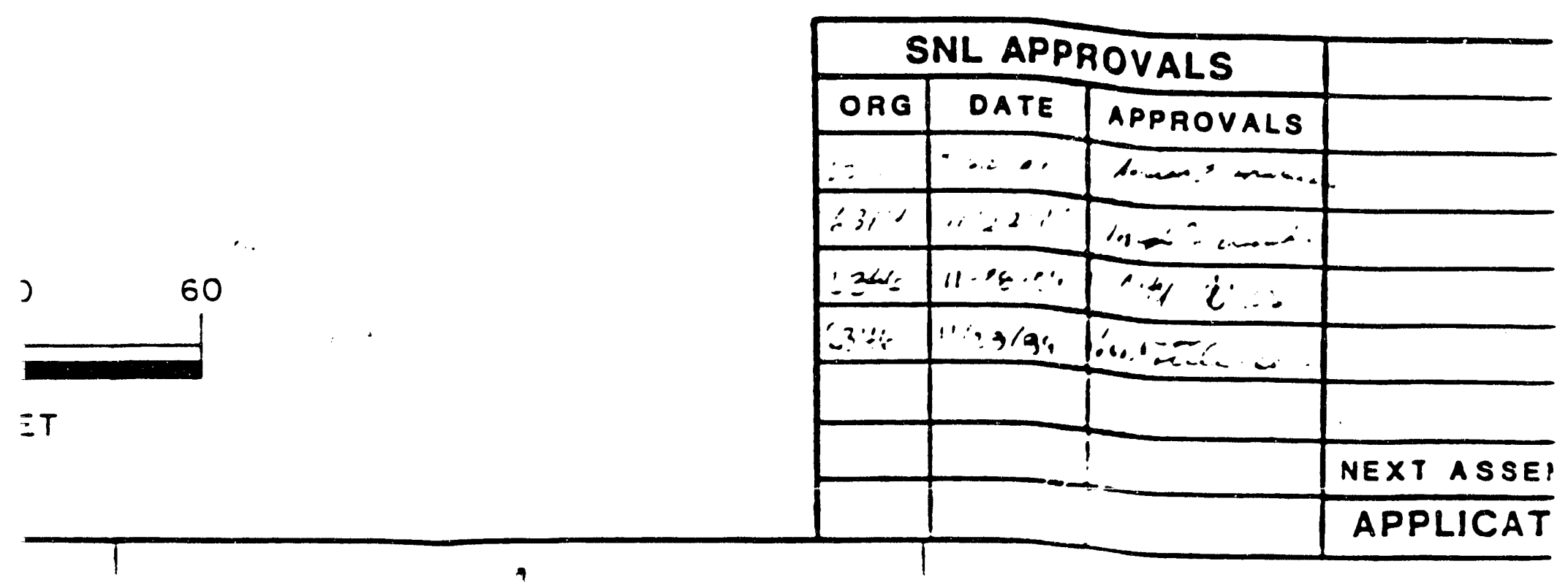



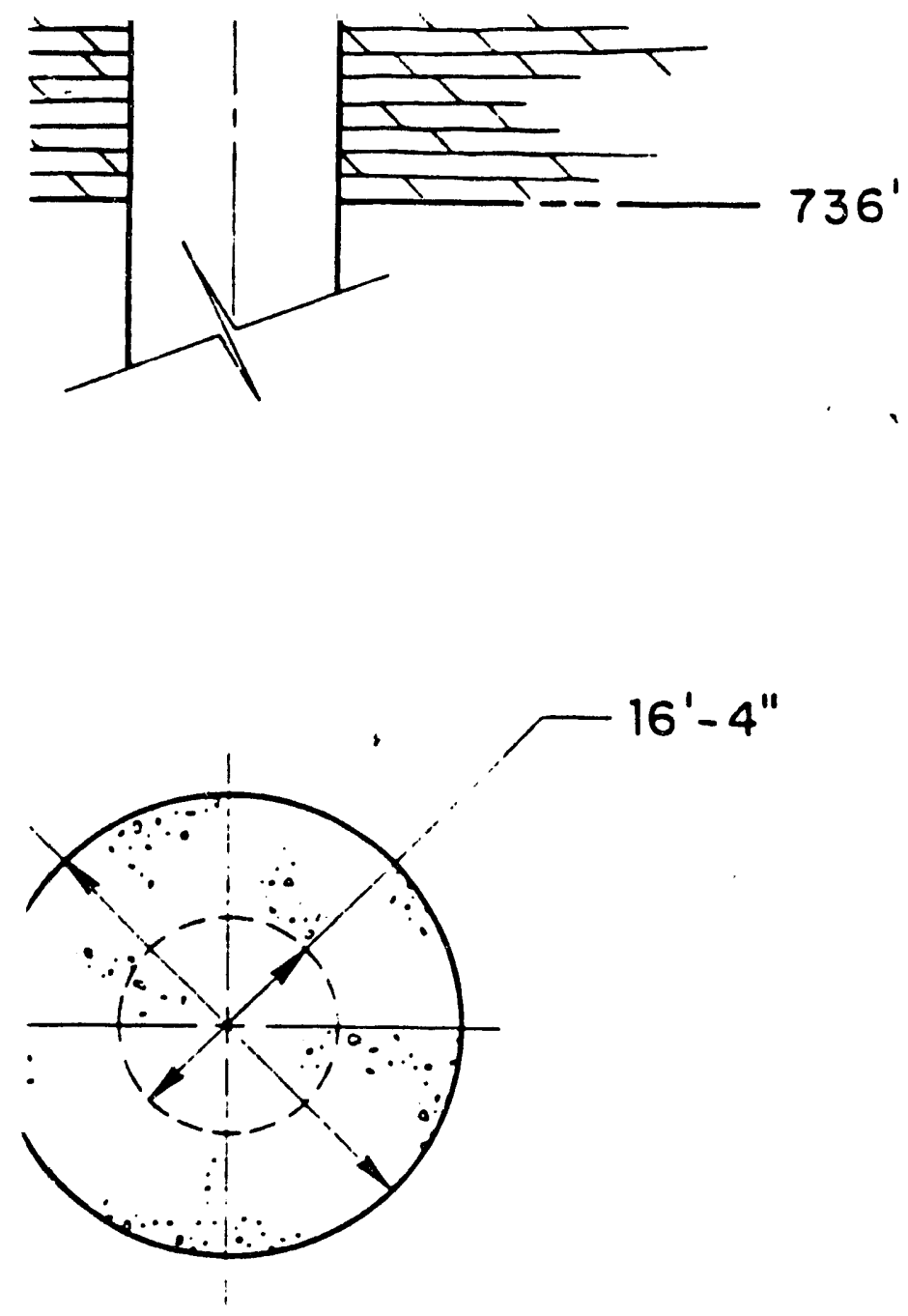

IECTION D-D

HAUST SHAFT
SUHRACLE ELEVATION THAT IS USED $\&$ 3,409'-0" (USGS 1927 NORTH AMERIC,

2. ALL STRATIGRAPHIC FEATURES ARE ACCORDING TO DATA COMPILED FRC ENERGY, OCTOBER 1986, WIPP DESIC REPORT, DOE-WIPP-86-010, BECHTEL FRANCISCO, CA.

3. THE WATER BEARING ZONE SEAL SYS CONSTRUCTED AT A DEPTH WHERE T BETWEEN THE TWO WATER-BEARING

4. ALL SHAFT DRAWINGS REPRESENT E) (I.E., WITHOUT LINERS) FOR BOTH EXI CONSTRUCTION. LINERS MUST BE RE EMPLACEMENT.

3. SHAFT DIAMETERS ARE REPRESENTE INFORMIATION OBTAINED FROM THE F SETS:

A. WASTE SHAFT. US DOE/WIPP DR; (BECHTEL, SAN FRANCISCO, CA); DRAWING 31-R-002-OID WESTINGi

B. AIR INTAKE SHAFT. US DOE/WIPP (BECHTEL, SAN FRANCISCO, CA) $\&$ DRAWING 33-R-029-34A (BECHTEL.

C. EXHAUST SHAFT. US DOE/WIPP D. (BECHTEL, SAN FRANCISCO, CA) $f$ DRAWING 35-R-002-OID (BECHTEL.

D. EXPLORATORY SHAFT. US DOE $N$ (BECHTEL, SAN FRANCISCO, CA) $A$ DRAWING 37-R-012 (BECHTEL, SAA

- EXPLORATORY SHAFT HAS SINCE BEE CONSTRUCTION \& SALT HANDLING SH

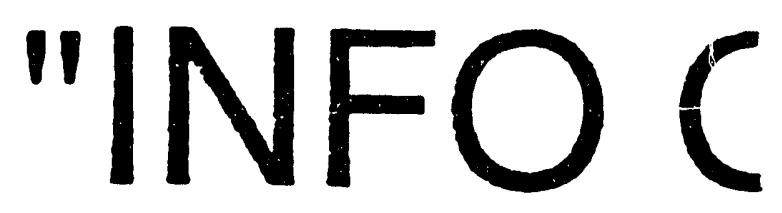

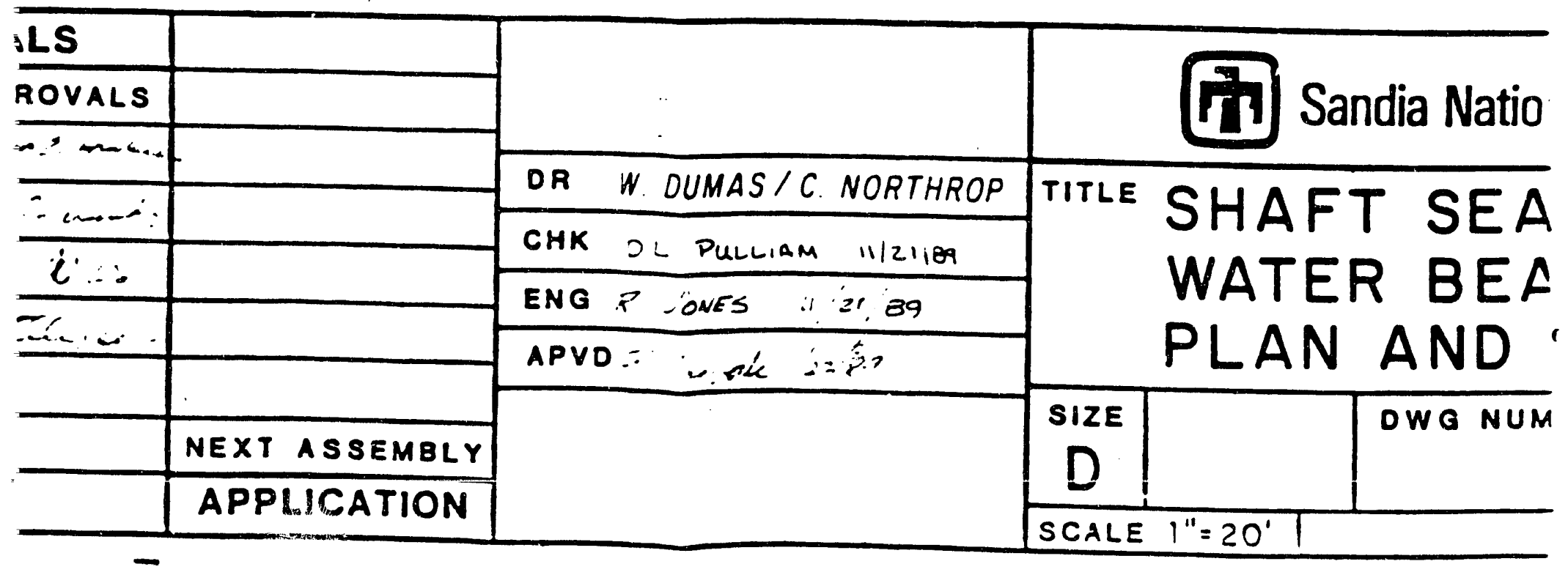


SURFACE ELEVATION THAT IS USED AS THE REFERENCE IS 3,409' -0" (USGS 1927 NORTH AMERICAN DATUM).

2. ALL STRATIGRAPHIC FEATURES ARE REPRESENTED ACCORDING TO DATA COMPILED FROM U.S. DEPARTMENT OF ENERGY, OCTOBER 1986, WIPP DESIGN VALIDATION FINAL REPORT, DOE-WIPP.86-010, BECHTEL NATIONAL INC., SAN FRANCISCO, CA.

3. THE WATER BEARING ZONE SEAL SYSTEM IS TO BE CONSTRUCTED AT A DEPTH WHERE THE SEAL IS CENTERED BETWEEN THE TWO WATER-BEARING MEMBERS.

4. ALL SHAFT DRAWINGS REPRESENT EXCAVATED DIAMETERS (I.E., WITHOUT LINERS) FOR BOTH EXISTING AND PLANNED CONSTRUCTION. LINERS MUST BE REMOVED BEFORE SEAL EMPLACEMENT.

5. SHAFT DIAMETERS ARE REPRESENTED ACCORDING TO INFORMATION OBTAINED FROM THE FOLLOWING DRAWING SETS:

A. WASTE SHAFT. US DOE/WIPP DRAWING 31-R-013-OID (BECHTEL, SAN FRANCISCO, CA) AND US DOE/WIPP DRAWING 31-R-002-OID (WESTINGHOUSE, CARLSBAD, NM).

B. AIR INTAKE SHAFT. US DOE/WIPP DRAWING 33-R-001-34A (BECHTEL, SAN FRANCISCO, CA) AND US DOE/WIPP DRAWING 33-R-029-34A (BECHTEL, SAN FRANCISCO, CA).

C. EXHAUST SHAFT. US DOE/WIPP DRAWING 35-R-001-OID (BECHTEL, SAN FRANCISCO, CA) AND US DOE/WIPP DRAWING 35-R-002-OID (BECHTEL, SAN FRANCISCO, CA).

D. EXPLORATORY SHAFT. US DOE/WIPP DRAWING 37-R-010 (BECHTEL, SAN FRANCISCO, CA) AND US DOE/WIPP DRAWING 37-R-012 (BECHTEL, SAN FRANCISCO, CA).

-EXPLORATORY SHAFT HAS SINCE BEEN CHANGED TO CONSTRUCTION \& SALT HANDLING SHAFT.

\section{II
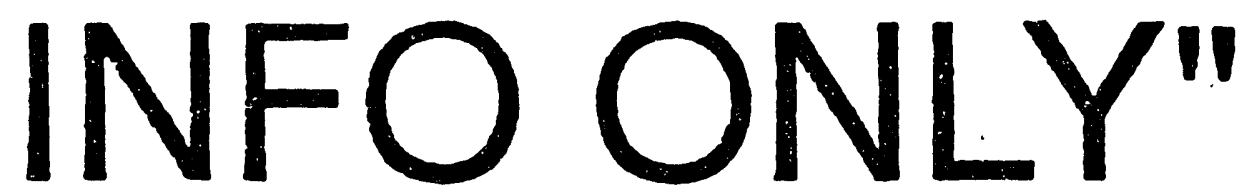

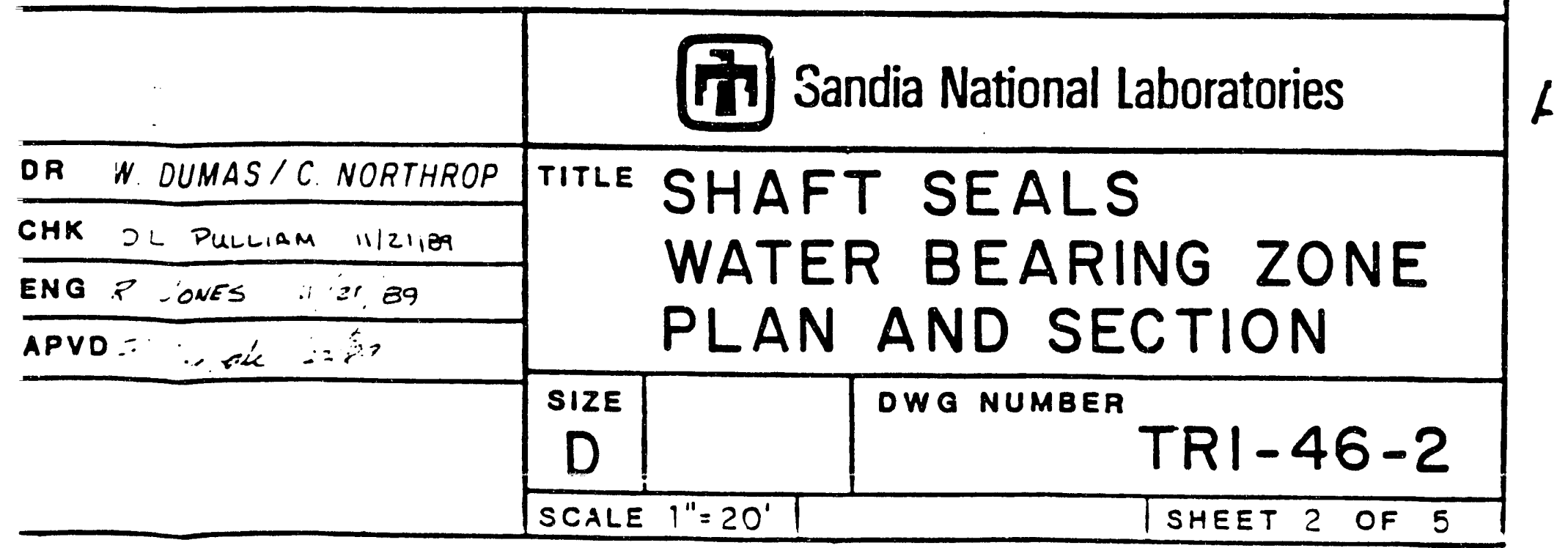




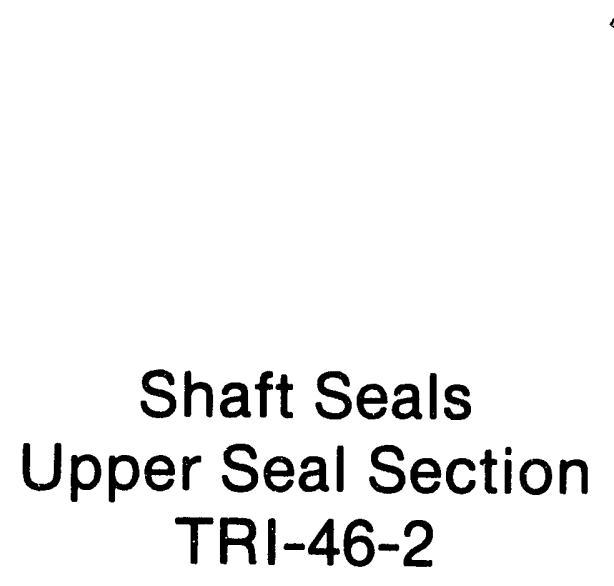




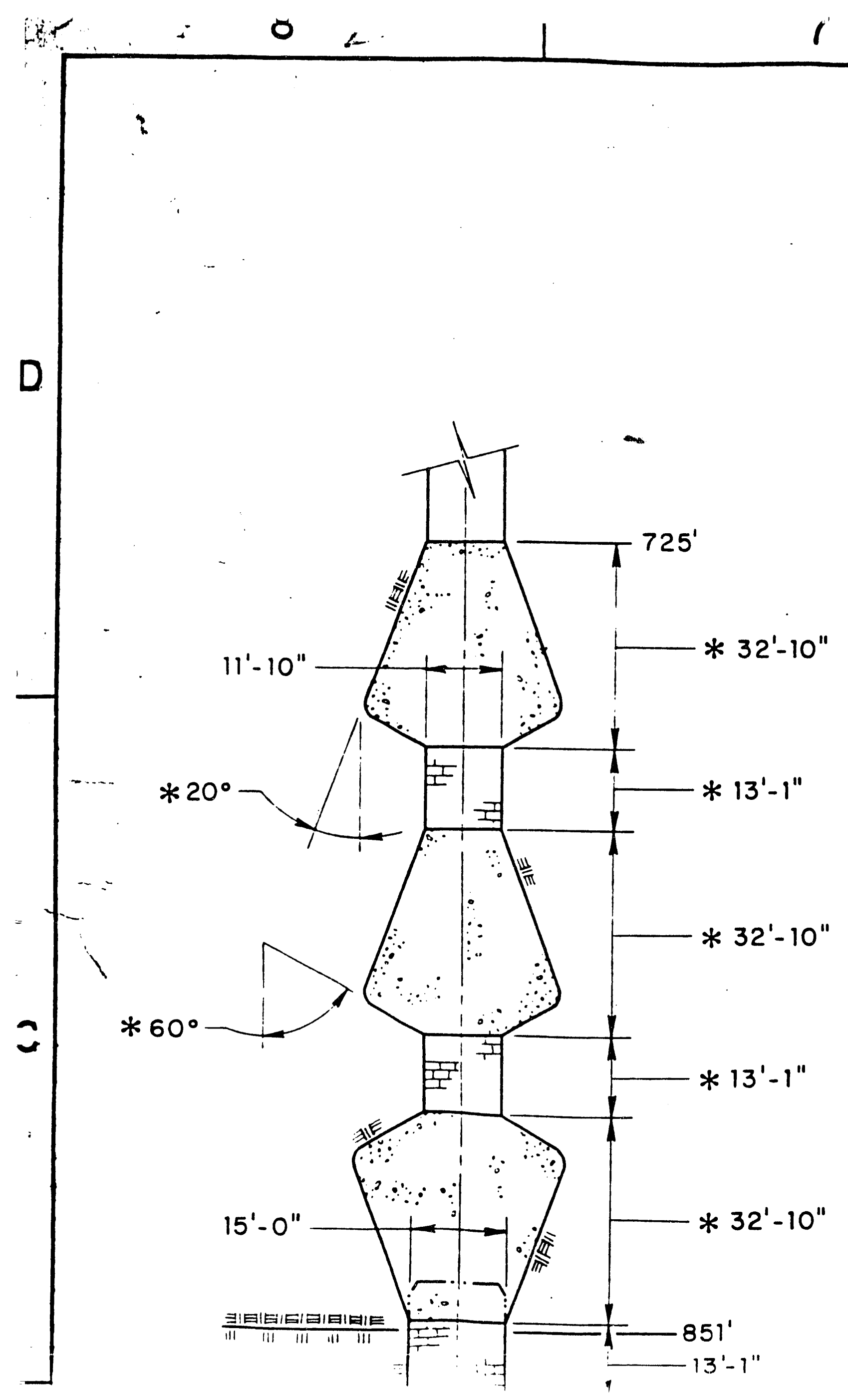




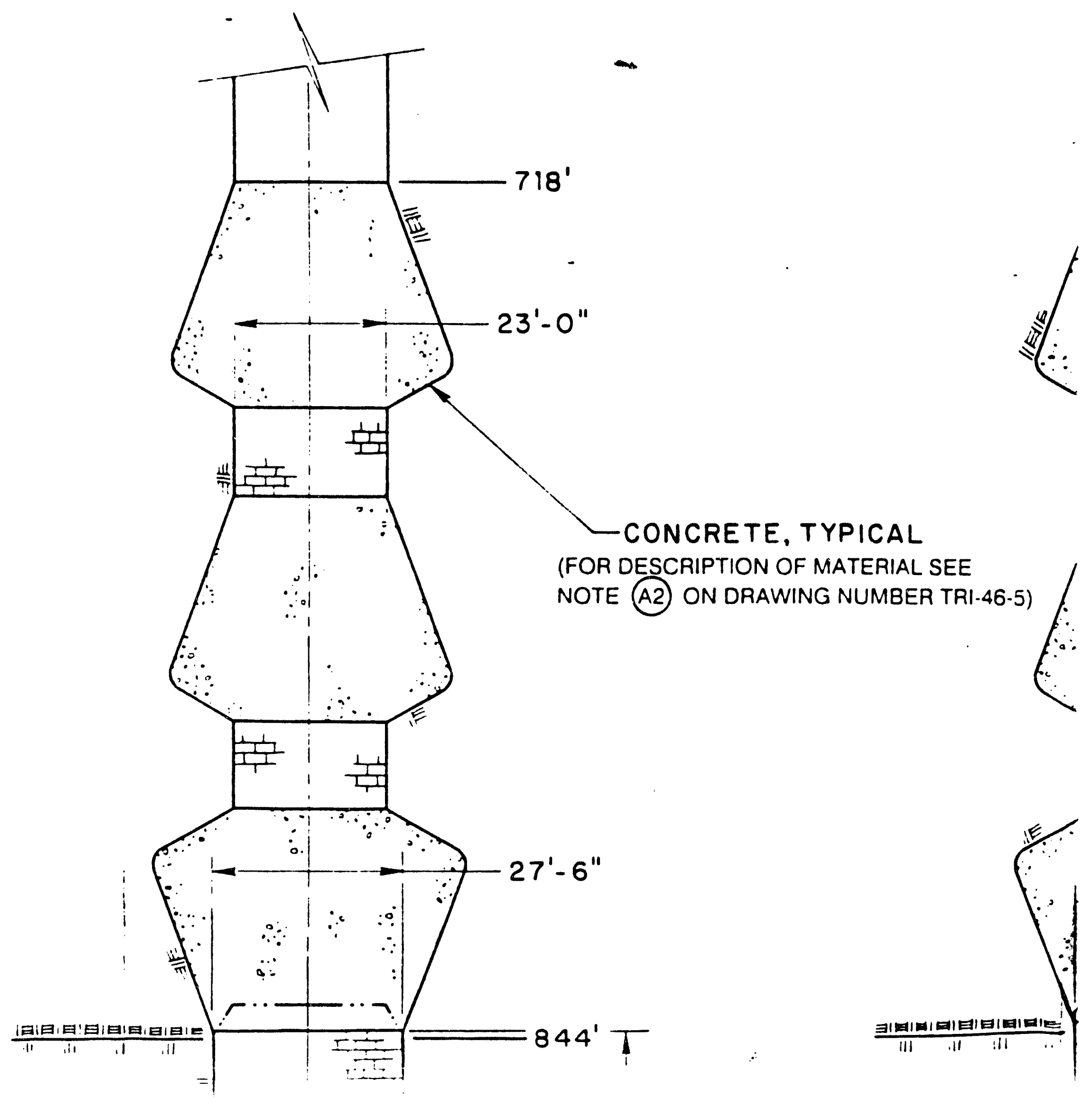




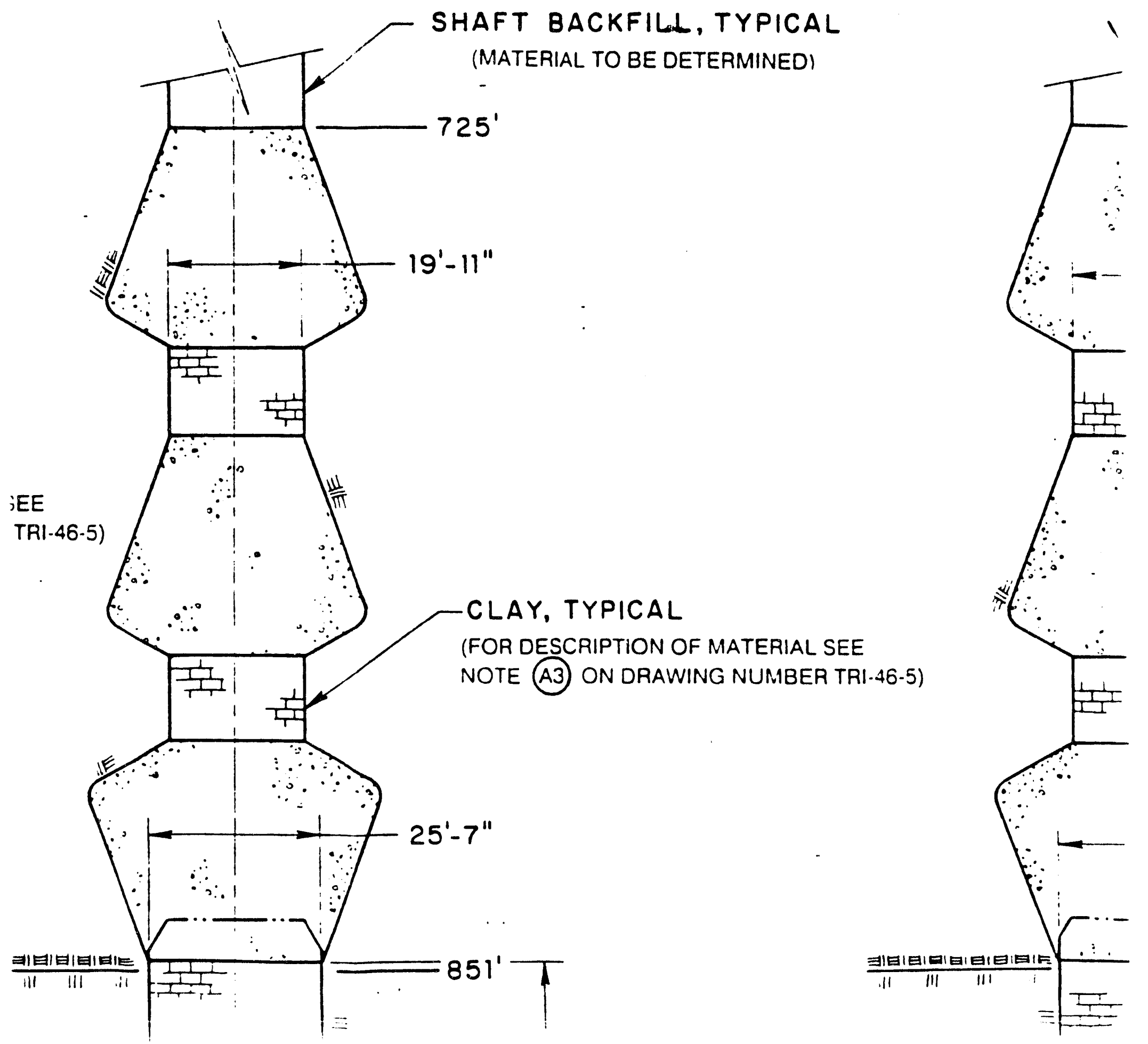





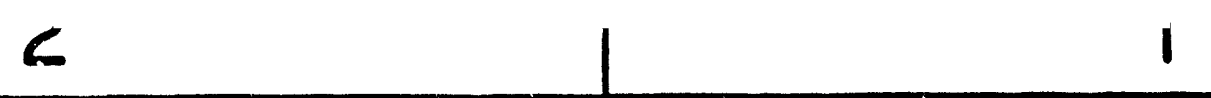

REVISIONS

\begin{tabular}{|l|l|l|l|}
\hline LTR & DESCRIPTION & DATE & APPROVED \\
\hline
\end{tabular}

\section{PICAL}

OF MATERIAL SEE

WING NUMBER TRI-46-5)

\section{NOTES}

1. DEPTH MEASUREMENTS INDICATE NUMBER OF FEET BELOW THE SURFACE. WHERE THE SURFACE EQUALS $0^{\circ}-0^{\prime \prime}$. THE SURFACE ELEVATION THAT IS USED AS THE REFERENGE IS 3.409'-0" (USGS 1927 NORTH AMERICAN DATUM).

2. ALL STRATIGRAPHIC FEATURES ARE REPRESENTED ACCORDING TO DATA COMPILED FROM U.S. DEPARTMENT OF ENERGY, OCTOBER 1986, WIPP DESIGN VALIDATION FINAL REPORT, DOE-WIPP-86-010. BECHTEL NATIONAL INC., SAN FRANCISCO. CA.

3. THE UPPER SHAFT SEAL SYSTEM IS TO BE CONSTRUCTED AT A DEPTH WHERE THE BOTTOM TWO COMPONENTS (I.E.. CLAY AND CONCRETE) RESIDE BELOW THE RUSTLER-SALADO CONTACT.

USTLER

IRMATION

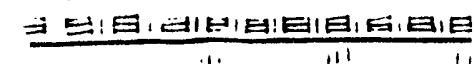

¿ALADO $=M \Delta^{-} \cap N$
4 ALL SHAFT DRAWINGS REPRESENT EXCAVATED DIAMETERS (1. E.. WITHOUT LINERS) FOR BOTH EXISTING AND PLANNED CONSTRUCTION. LINERS AND KEYS MUST BE REMOVED BEFORE SEAL EMPLACEMENTS. 


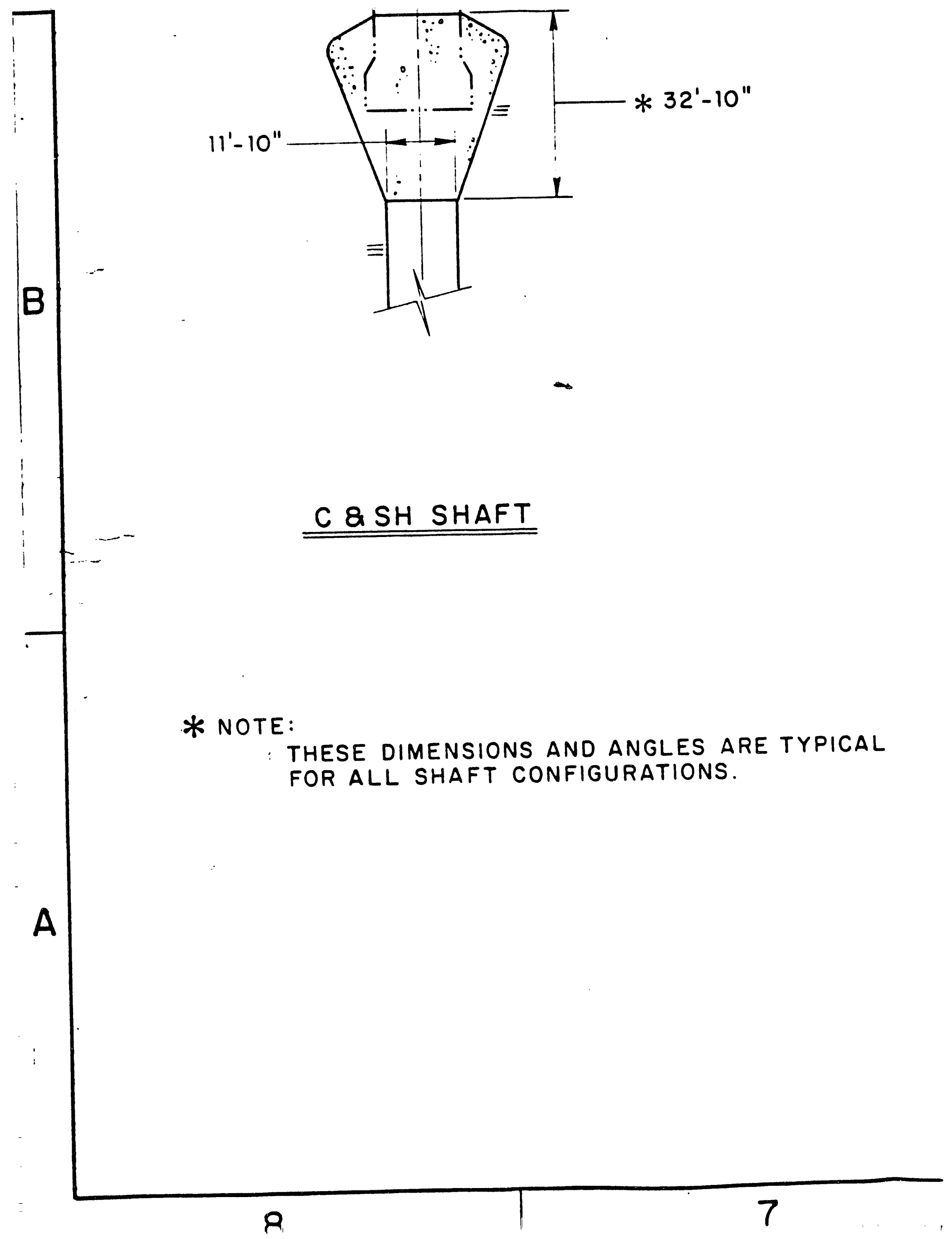


* 32'-10"

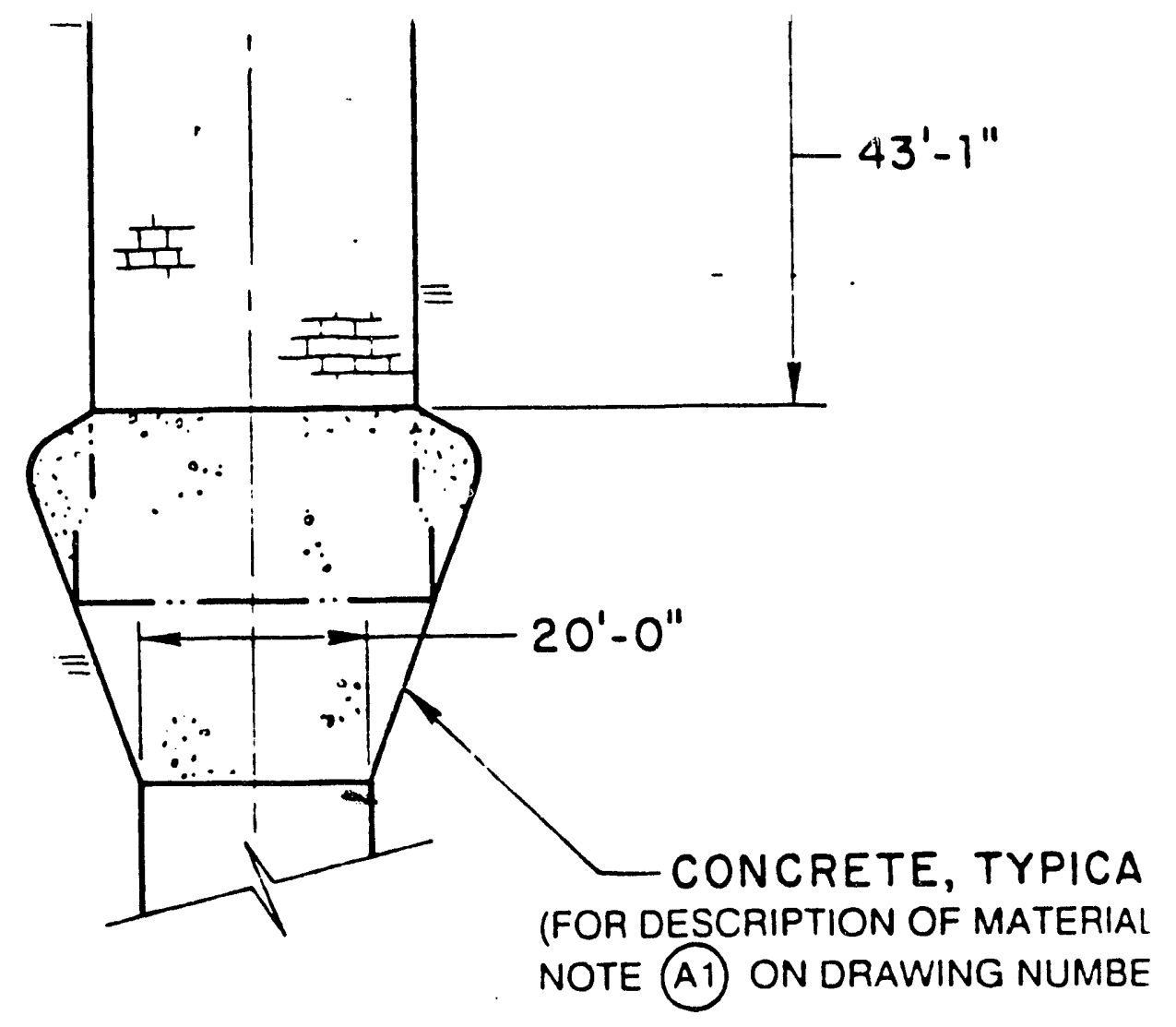

WASTE SHAFT

!GLES ARE TYPICAL ATIONS. 


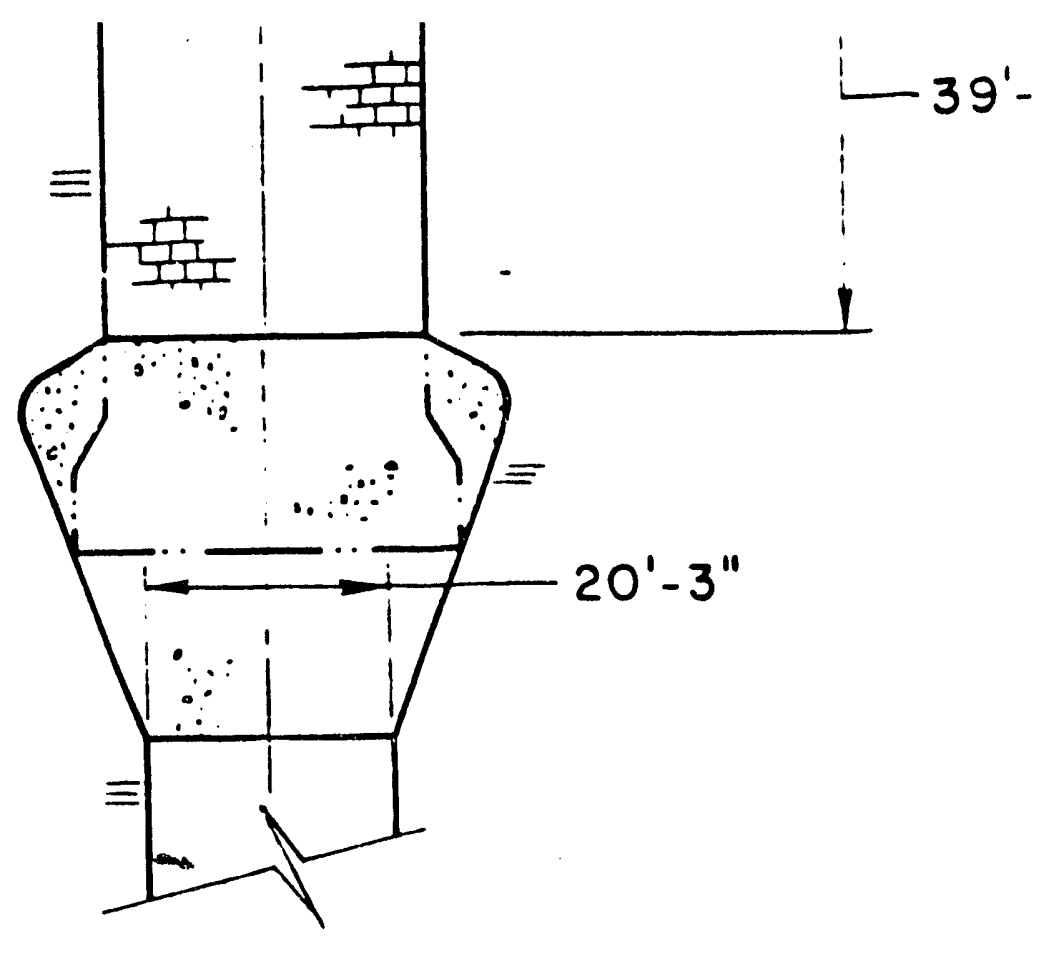
NOTE (A) ON DRAWING NUMBER TRI-46-5)

AIR INTAKE SHAFT

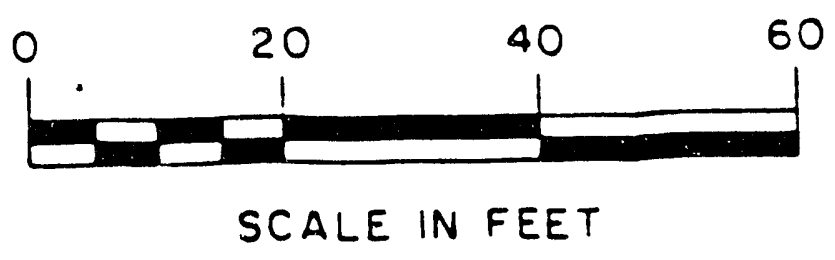




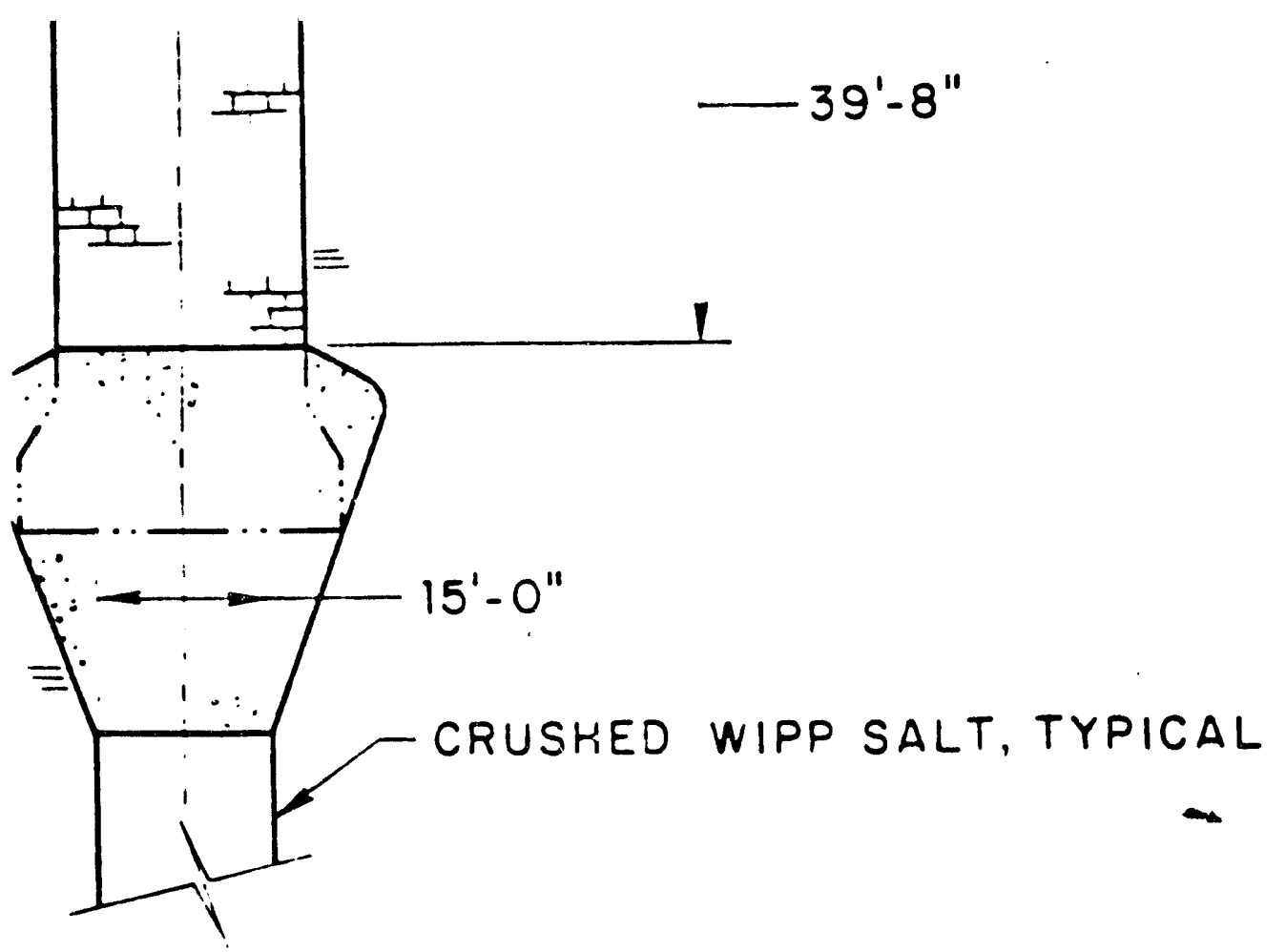

(..- ..) THE KEYS ARE R INFORIIATION OBTAINED SETS:

A. WASTE SHAFT. USD (BECHTEL. SAN FRAI DRAWING 31-R-CO2-O

B. AIR INTAKE SHAFT. U (BECHTEL. SAN FRAP DRAWING 33-R-029-3.

C. EXHAUST SHAFT. US (BECHTEL. SAN FRA! DRAWING 35-R-002-C

D. EXXPLORATORY SHA (BECHTEL. SAN FRAI DRAWING 37-R-012 (E

-EXPLORATORY SHAFT 1 CONSTRUCTION \& SALT

\section{IAUST SHAFT}

\section{"INFC}

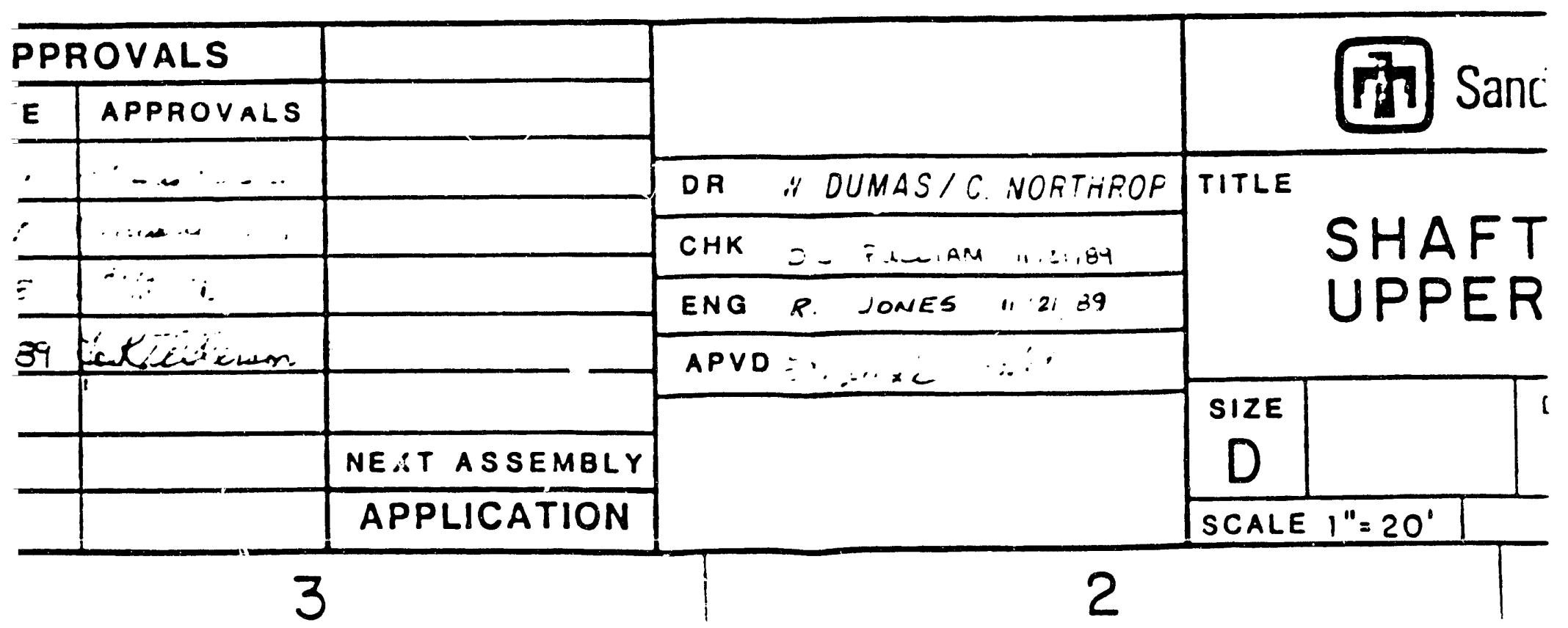


$\bullet \cdot+\cdot \cdot m \cdot \cdot \omega$

$3^{\prime}-8^{\prime \prime}$

SALT, TYPICAL
¿. ENOWUU U M

(..-..) THE KEYS ARE REPRESENTED ACCORDING TO INFORI.IATION OBTAINED FROM THE FOLLOWING DRAWING SETS:

A. WASTE SHAFT. US DOE, WIPP DRAWING 31-R-013-OID (BECHTEL. SAN FRANCISCO. CA) AND US DOE!WIPP DRAWING 31-R-C02-OID (WESTINGHOUSE. CARLSBAD. NM).

B. AIR INTAKE SHAFT. US DOE. WIPP DRAWING 33-R-001-34A (BECHTEL. SAN FRANCISCO. CA) AND US DOE. WIPP DRAWING 33-R-029-34A (BECHTEL. SAN FRANCISCO. CA).

C. EXHAUST SHAFT. US DOE:WIPP DRAWING 35-R-001-OID (BECHTEL. SAN FRANCISCO, CA) AND US DOE ; WIPP DRAWING 35-R-002-OID (BECHTEL. SAN FRANCISCO. CA).

D. EXPLORATORY SHAFT. US DOE 'WIPP DRAWING 37-R-010 (BECHTEL. SAN FRANCISCO. CA) AND US DOE /WIPP DRAWING 37-R-012 (BECHTEL. SAN FRANCISCO, CA).

- EXPLORATORY SHAFT has SINCE BEEN CHANGED TO CONSTRUCTION \& SALT HANDLING SHAFT.

\section{"INFO ONLY"}

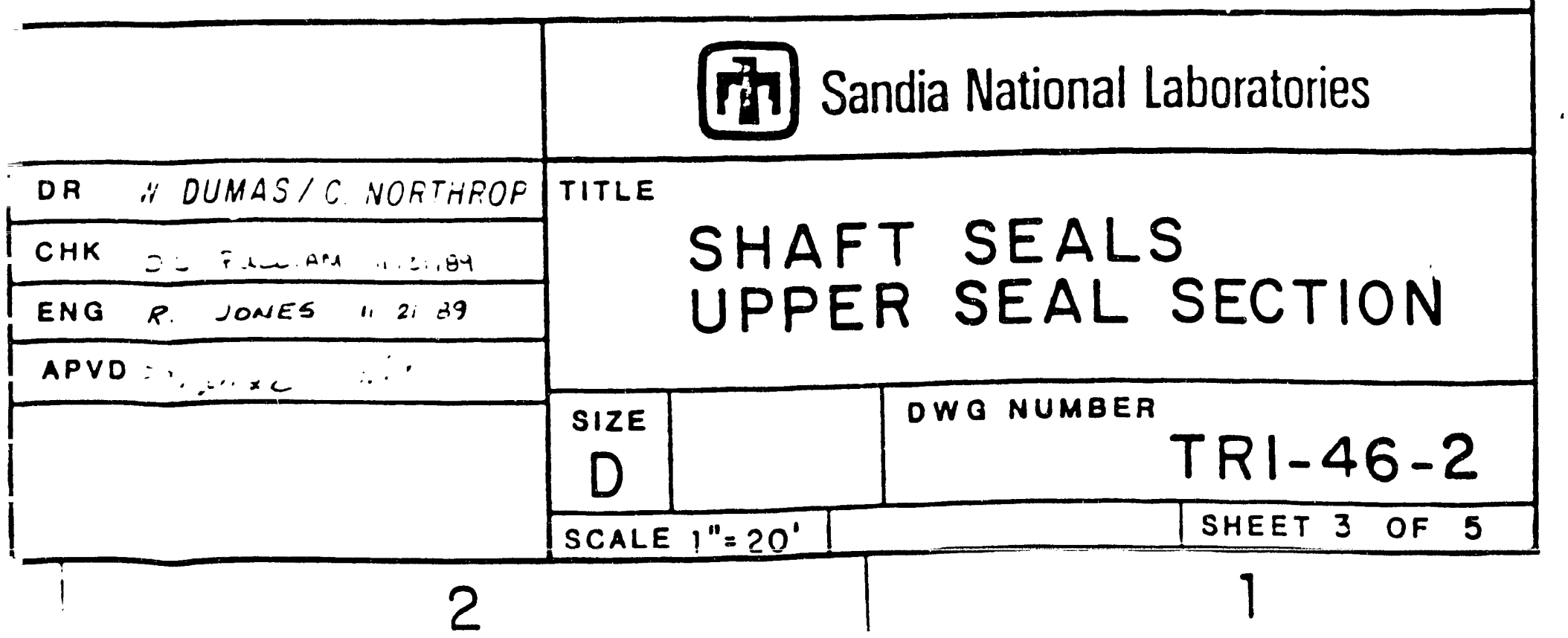




$$
=1
$$


D

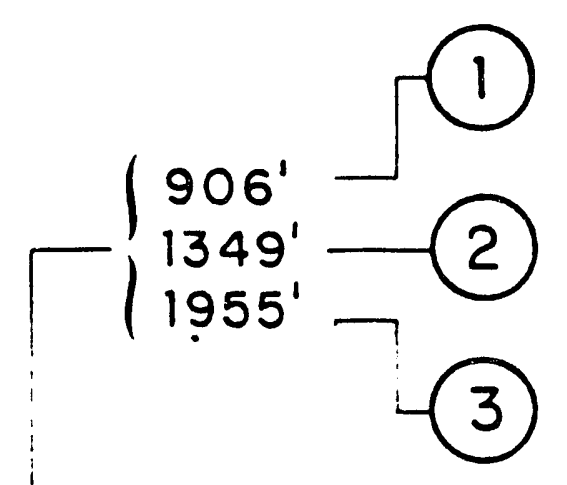

* $32^{\prime}-10^{\prime \prime}$

20'-
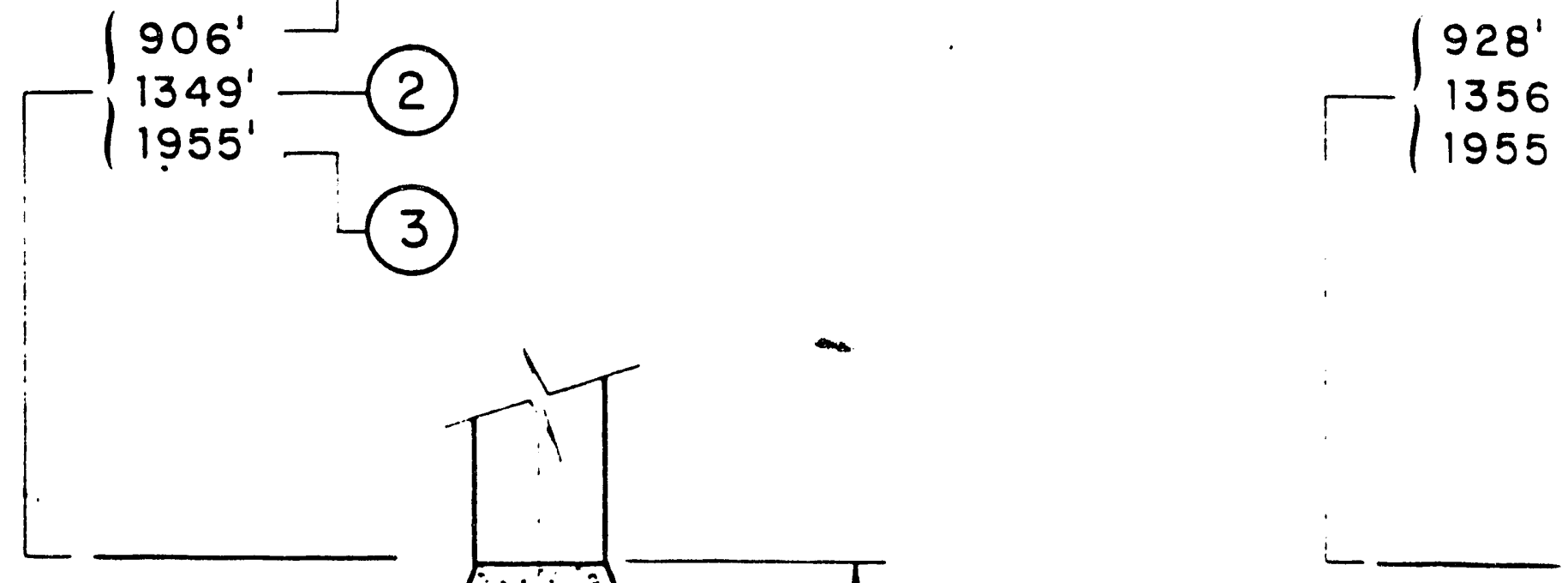

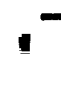

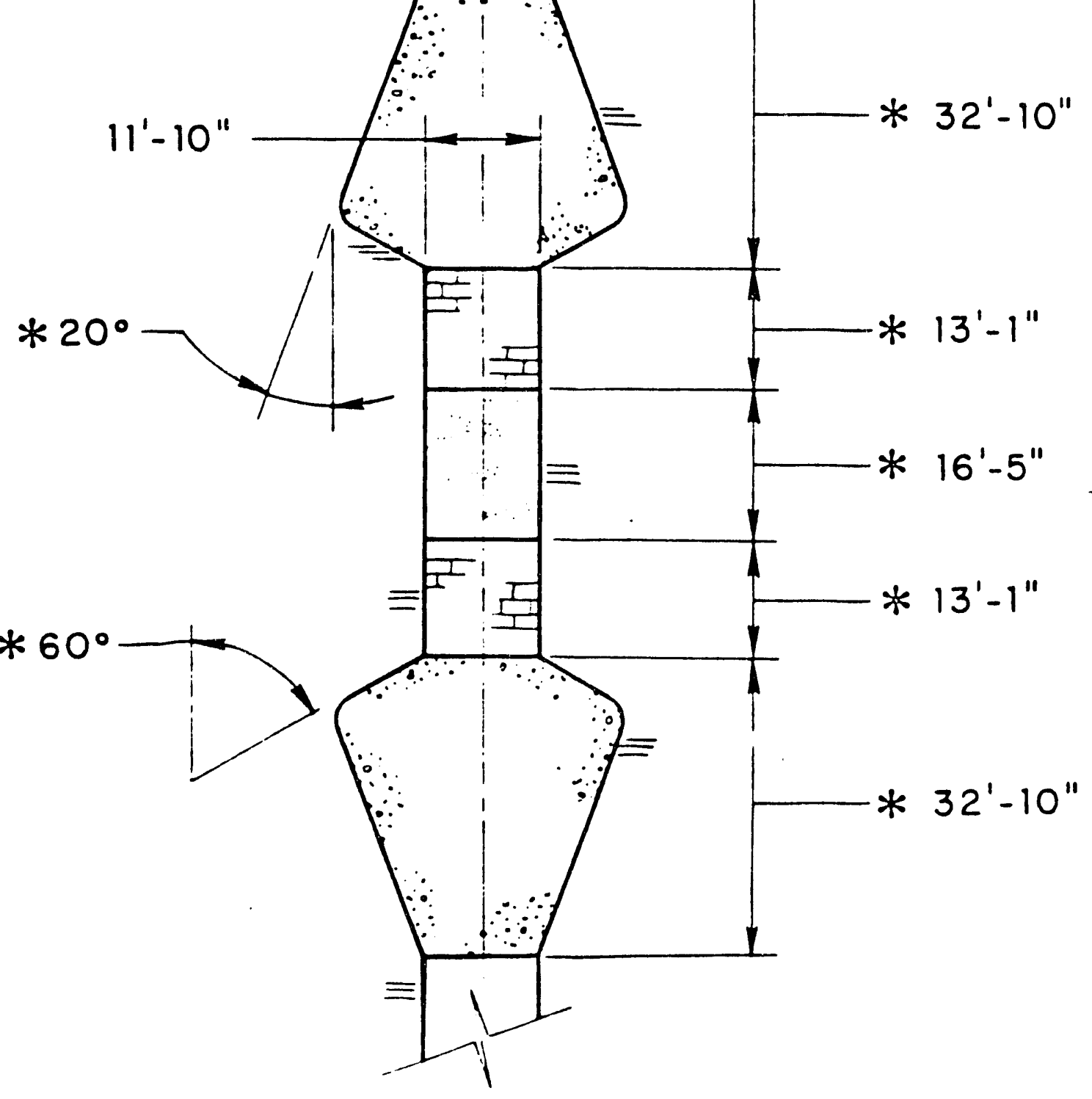




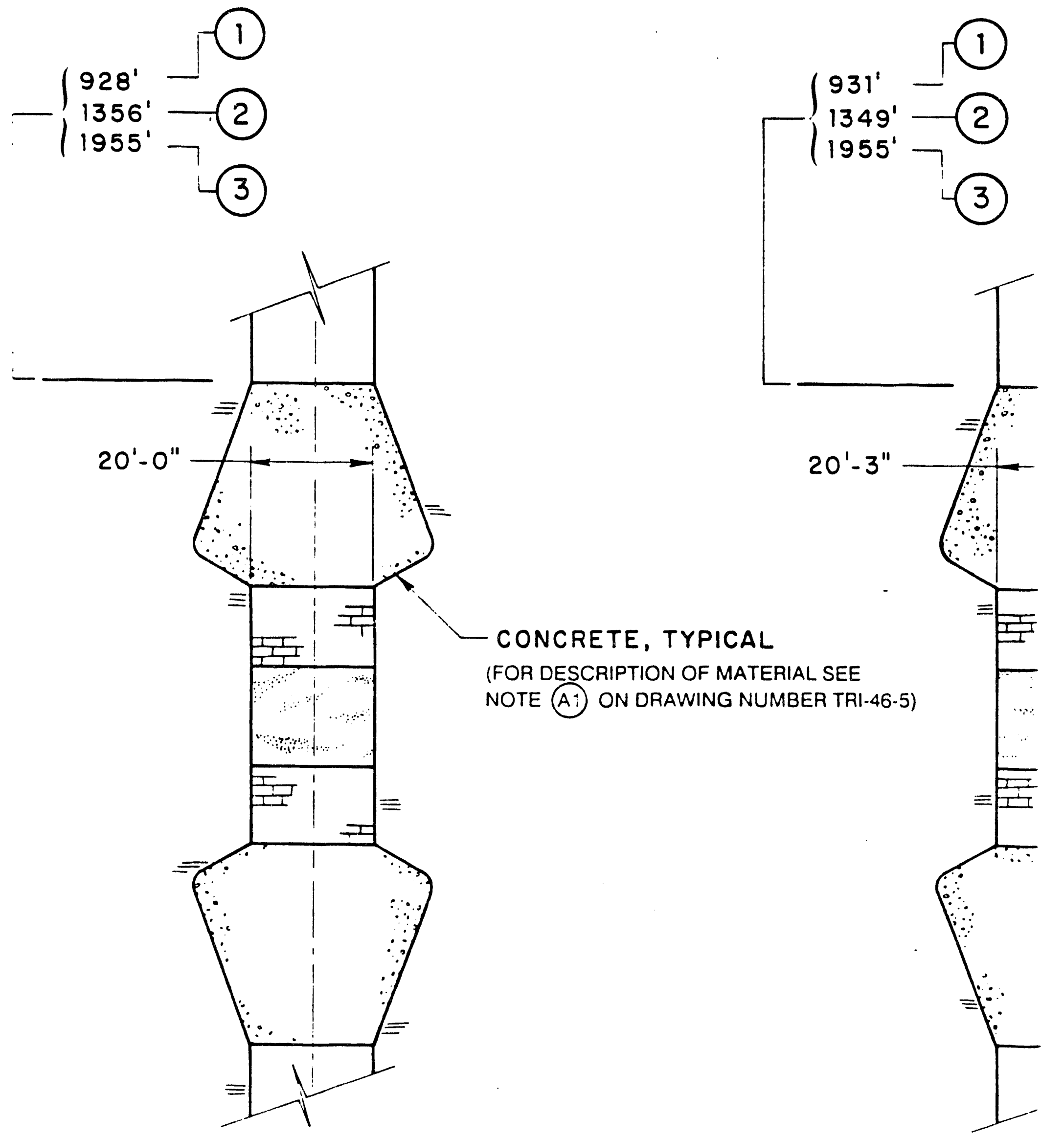


(1)

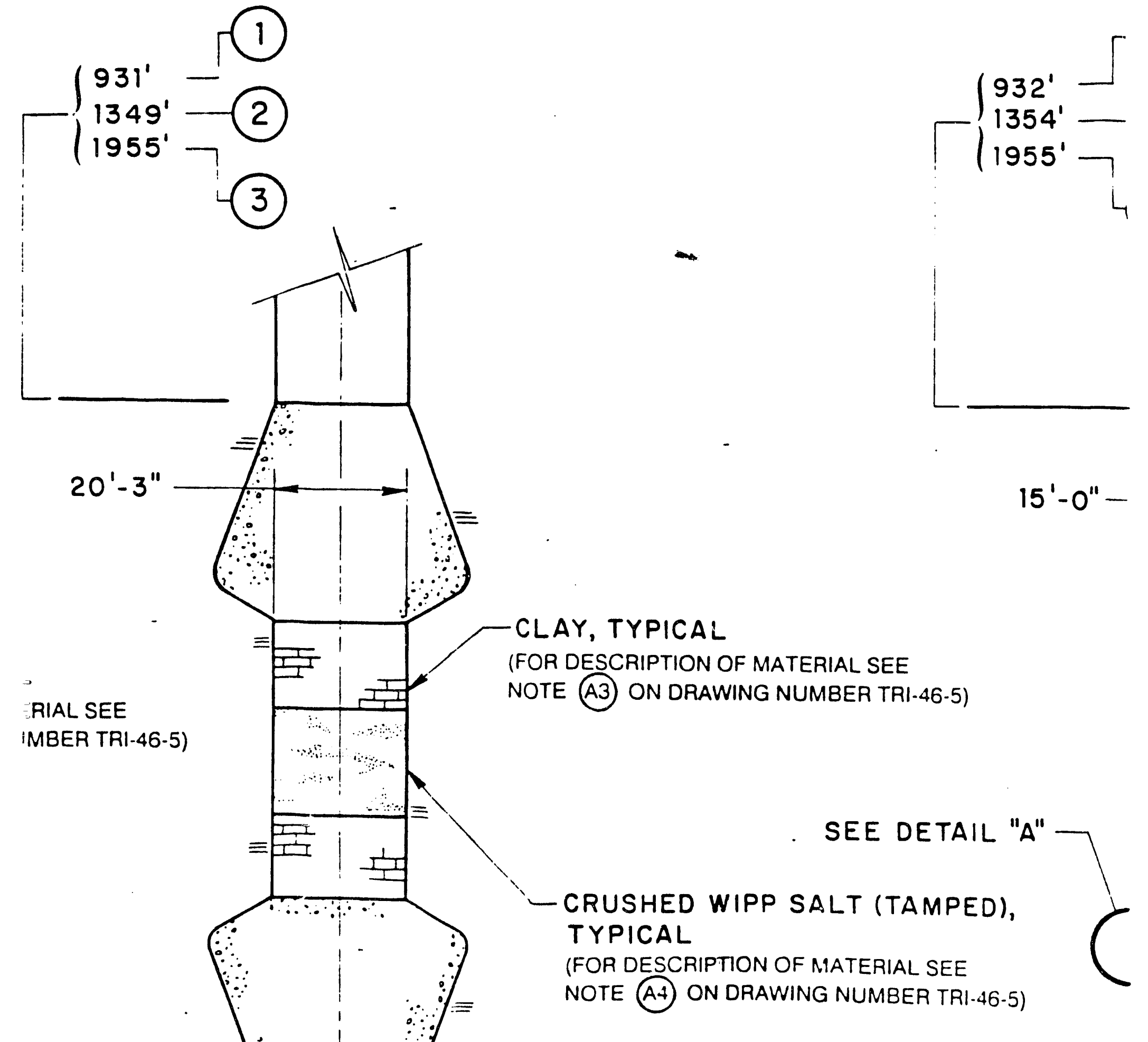




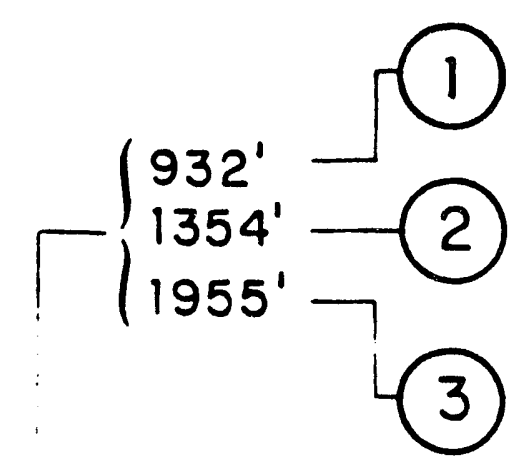

SEE

R TRI-46-5)
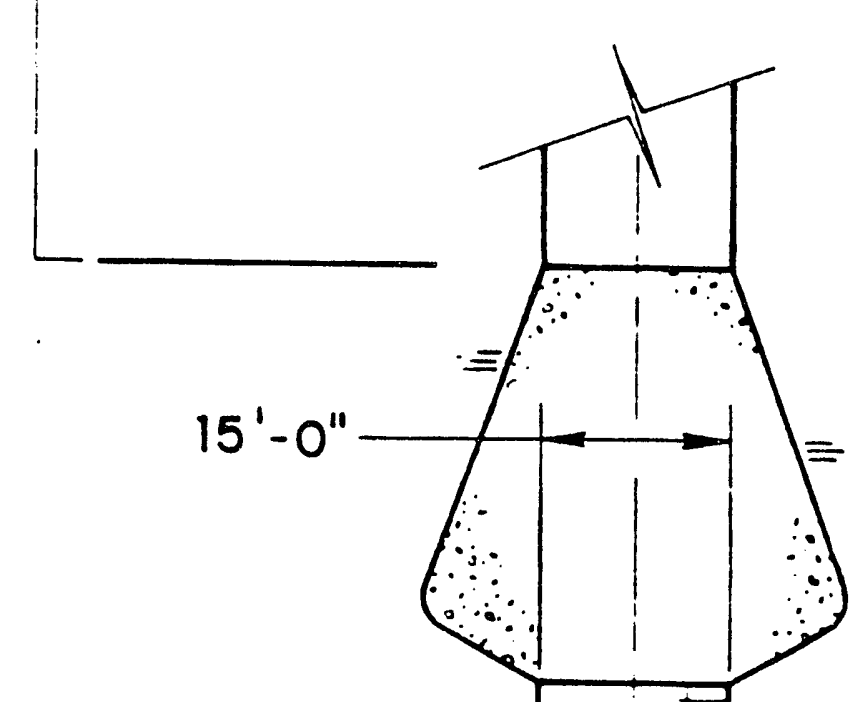

$15^{\prime}-0^{\prime \prime}$

E DETAIL "A"

(TAMPED),

ERIAL SEE

IMBER TRI-46-5)

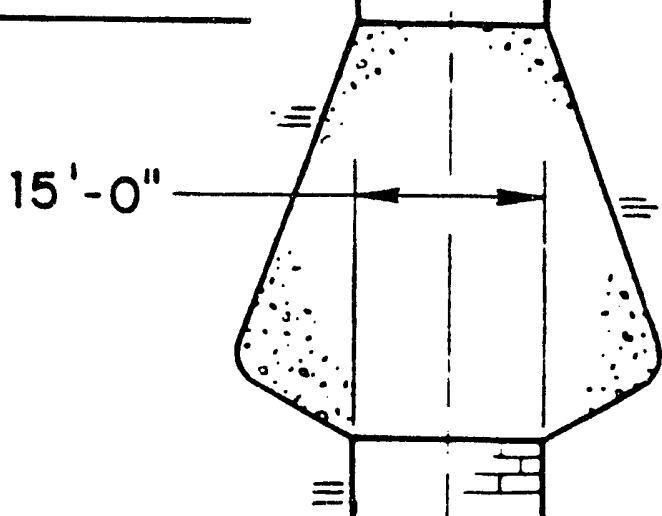




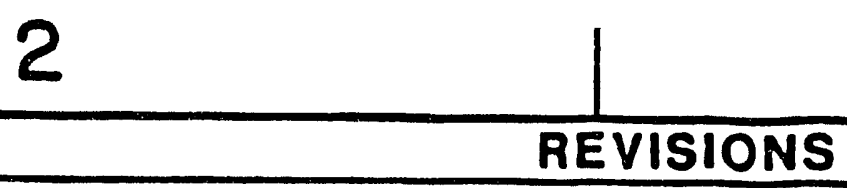

\begin{tabular}{l} 
LTR DESCRIPTION \\
\hline
\end{tabular}

DATE

APPROVED

D WIPP SALT (TAMPED), ZIPTION OF MATERIAL SEE ON DRAWING NUMBER TRI-46-5)

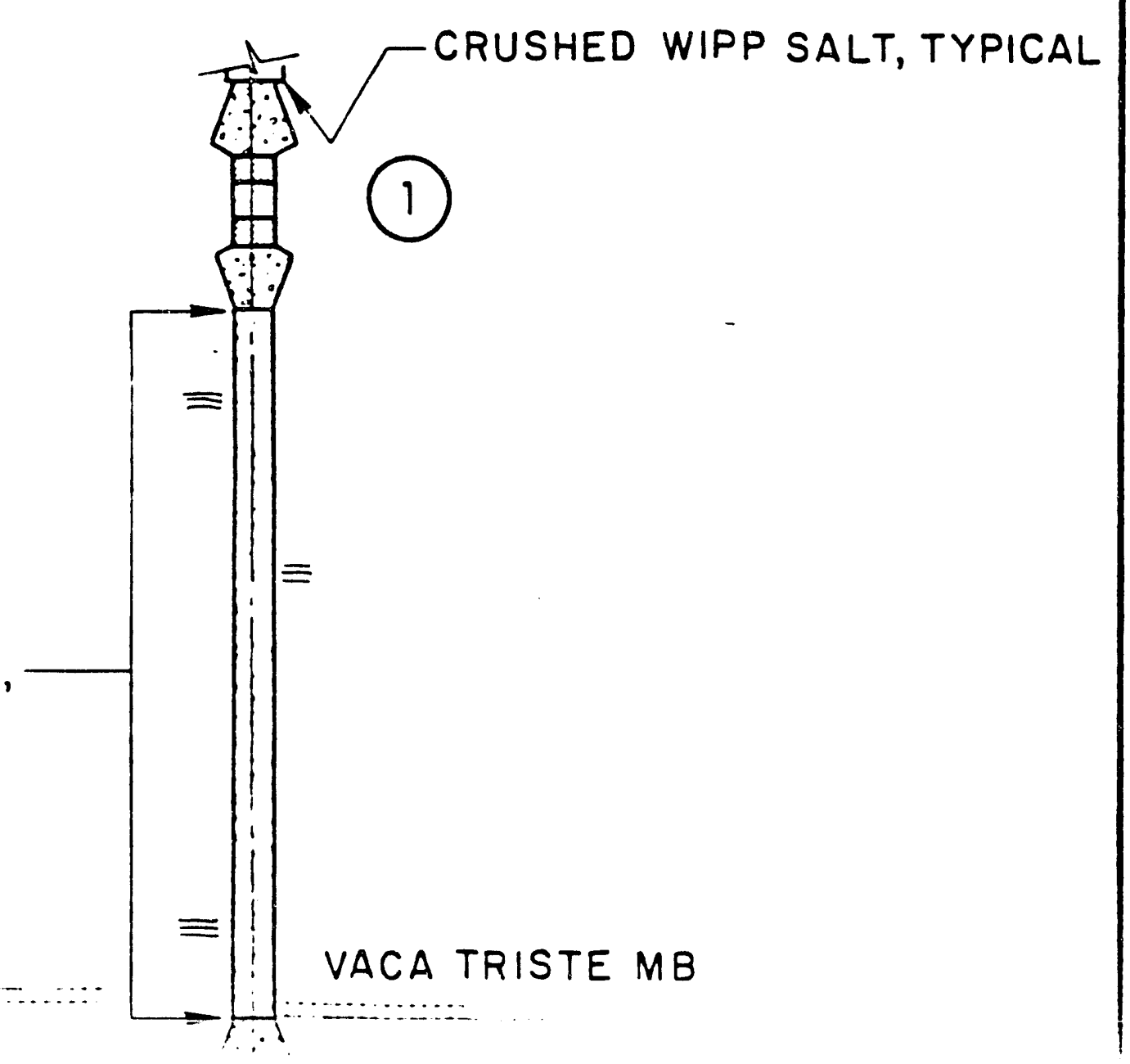




\section{NOTES}

1. DEPTH MEASUREMENTS INDICATE NUMBER OF FEET BELOW THE SURFACE. WHERE THE SURFACE EQUALS $0^{\prime}-0^{\prime \prime}$. THE SURFACE ELEVATION THAT IS USED AS THE REFERENCE IS $3.409^{\circ} \cdot{ }^{\prime \prime}$ (USGS 1927 NORTH AMERICAN DATUM).

2. ALL STRATIGRAPHIC FEATURES ARE REPRESENTED

ACCORDING TO DATA COMPILED FROM U.S. DEPARTMENT OF

ENERGY, OCTOBER 1986. WIPP DESIGN VALIDATION FINAL REPORT, DOE.WIPP.86-010, BECHTEL NATIONAL INC., SAN FRANCISCO. CA.

3. THE TOP COMPONENTS OF THE LOWER SHAFT SEALS ARE TO BE CONSTRUCTED AT A DEPTH NO LESS THAN 9'-10" BELOW THE UPPER SHAFT SEAL SYSTEM.

4. THE MIDDLE COMPONENTS OF THE LOWER SHAFT SEALS ARE TO BE CONSTRUCTED JUST BELOW THE VACA TRISTE MARKER BED.

5. THE BOTTOM COMPONENTS OF THE LOWER SHAFT SEALS ARE TO BE CONSTRUCTED AT THE 1955'-0" LEVEL FOR ALL SHAFTS.

6. ALL SHAFT DRAWINGS REPRESENT EXCAVATED DIAMETERS FOR BOTH EXISTING AND PLANNED CONSTRUCTION. THIS PORTION OF THE SHAFT IS UNLINED.

7. SHAFT DIAMETERS ARE REPRESENTED ACCORDING TO INFORMATION OBTAINED FROM THE FOLLOWING DRAWING SETS: (BECHTEL. SAN FRANCISCO. CA) AND US DOE/WIPP DRAWING 31-R-OC2-OID (WESTINGHOUSE, CARLSBAD. NM).
A. WASTE SHAFT. US DOE/WIPP DRAWING 31-R-013-OID
B. AIR INTAKE SHAFT. US DOE/WIPP DRAWING 33-R-001-34A (BECHTEL, SAN FRANCISCO, CA) AND US DOE/WIPP DRAWING 33-R-029-34A (BECHTEL, SAN FRANCISCO. CA).
C. EXHAUST SHAFT. US DOE/WIPP DRAWING 35-R-001-OID (BECHTEL. SAN FRANCISCO, CA) AND US DOE WIPP DRAWING 35-R-002-OID (BECHTEL, SAN FRANCISCO, CA).
D. EXPLORATORY SHAFT. US DOE/WIPP DRAWING 37-R-010 (BECHTEL, SAN FRANCISCO, CA) AND US DOENIPP DRAWING 37-R-012 (BECHTEL, SAN FRANCISCO. CA).

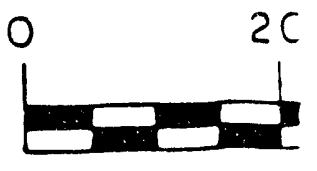

-EXPLORATORY SHAFT HAS SINCE BEEN CHANGED TO SC, CONSTRUCTION \& SALT HANDLING SHAFT. 
$1)$ 110

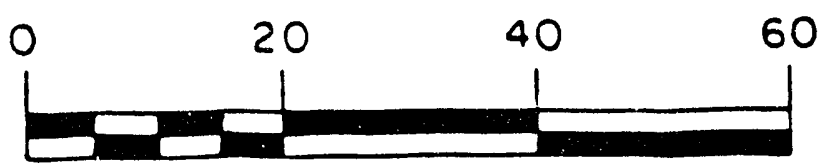

60

SCALE IN FEET 


\section{EXHAUST SHAFT}

SCALE: $I^{\prime \prime}=20^{\prime}$

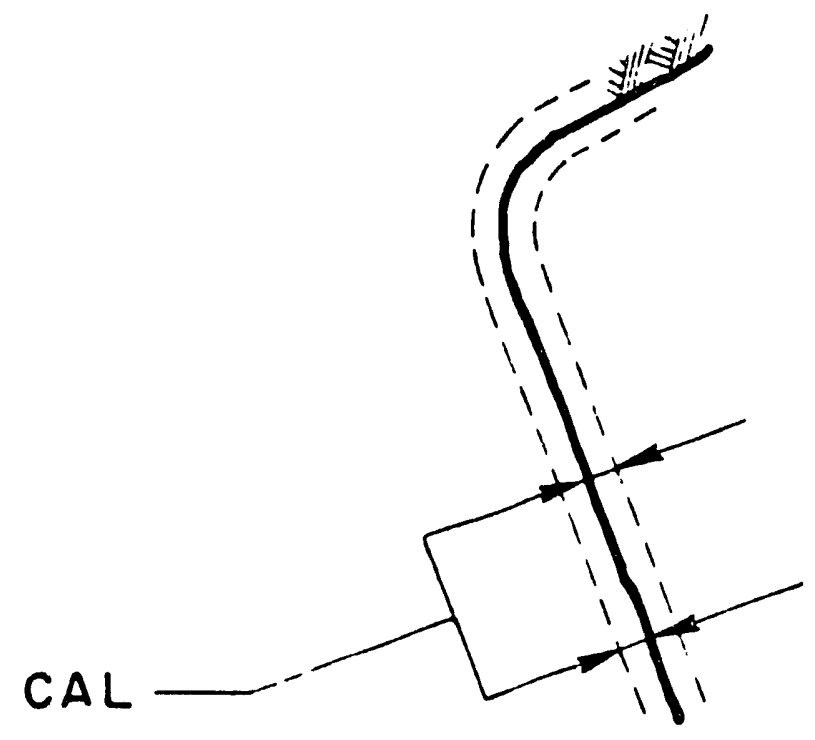
CRUSHED WIPP SALT (TAMPE - TYPICAL

(FOR DESCRIPTION OF MATERIAL SEE NOTE A4 ON DRAWING NUMBER TRI-4f

$\frac{\text { DETAIL "A" }}{\text { NO SCALE }}$

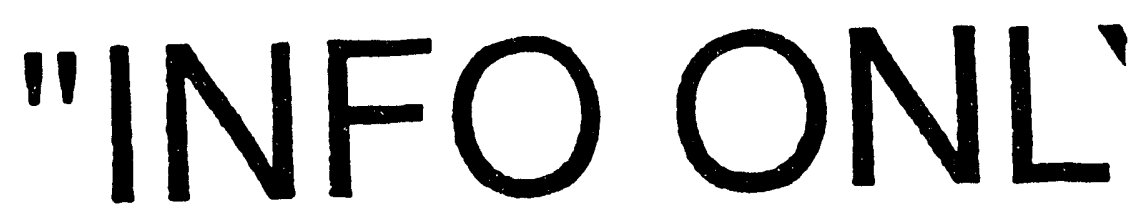

\begin{tabular}{|c|c|c|c|c|}
\hline \multicolumn{3}{|c|}{ SNL APPROVALS } & & \\
\hline ORG & DATE & APPROVALS & & \\
\hline 3,3 & $\therefore \theta^{\circ}$ & 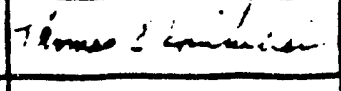 & & DR W. DUPAS / C. NORTH \\
\hline 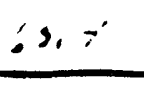 & $.2 \div 3 i$ & Gxpásis & & CHK DL. PULLIAM IIIZI:Z \\
\hline 6046 & $11 \cdot 28 \cdot 84$ & !ㅏ & & ENG $R$. JONES "21.8S \\
\hline$\because+1$ & $11 / 23 / 74$ & inexticis an & & APVD $=\because 4+x=\therefore a$ \\
\hline & & & NEXT A S SEMBLY & \\
\hline & & & APPLICATION & \\
\hline
\end{tabular}


D WIPP SALT (TAMPED), $-$

RIPTION OF MATERIAL SEE

ON DRAWING NUMBER TRI.45-5)

COMPOSITE LAYOUT

SCALE: $1^{\prime \prime}=100^{\prime}$

(3)

CRUSHED WIPP SALT

(DRIFT BACKFILL), TYPICAL

(FOR DESCRIPTION OF MATERIAL SEE

NOTE A5 ON DRAWING NUMBER TRI-46-5)

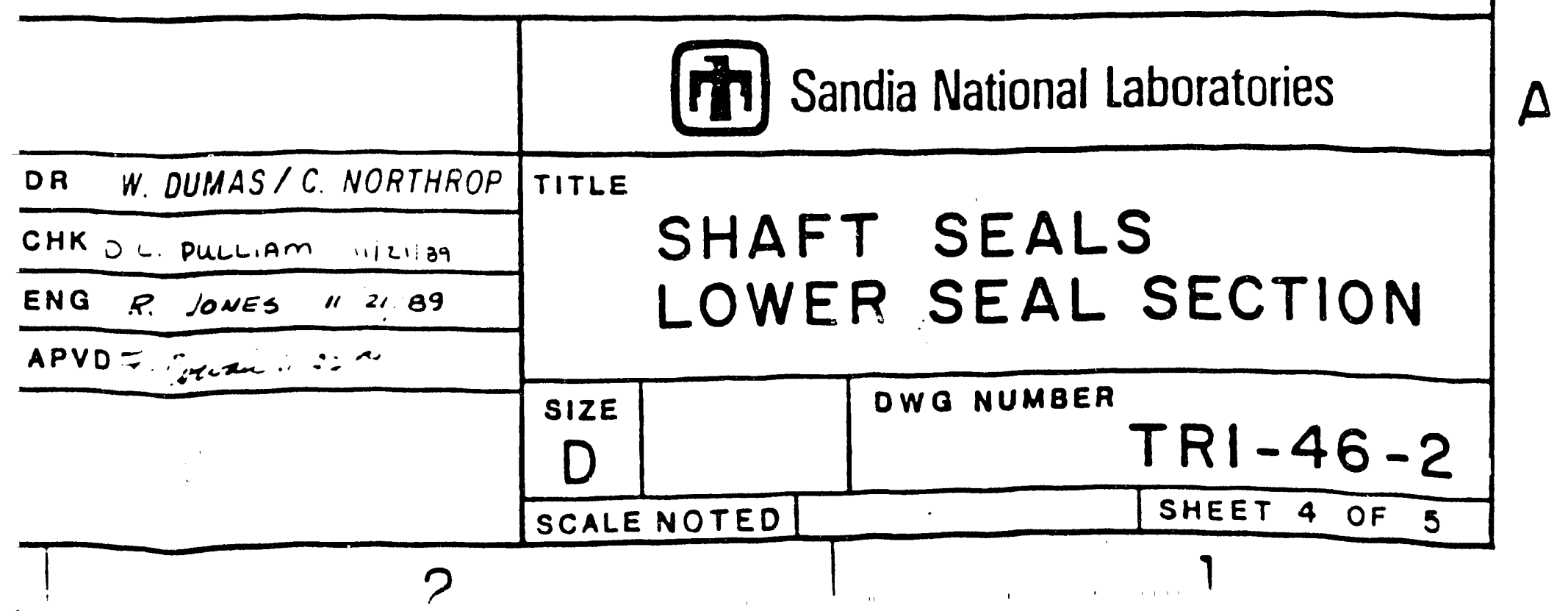




\section{C \& SH SHAFT}

\section{SHAFT SEAL SUMMARY}

\begin{tabular}{|c|c|c|c|}
\hline $\begin{array}{l}\text { SEAL : } \\
\text { SYSTE:A }\end{array}$ & MATERIAL & $\begin{array}{l}\text { AXIMUM } \\
\text { METER (h.) }\end{array}$ & $\begin{array}{l}\text { SEAL INTERVAL } \\
\text { BELOW GROUND } \\
\text { SURFACE (H) }\end{array}$ \\
\hline $\begin{array}{l}\text { CAP COLLAR. } \\
\text { BACKFILL } \\
\text { AND'PLUG }\end{array}$ & TO BE SPECIFIED & 11.8 & $0.0 \cdot 629.0$ \\
\hline \multirow{2}{*}{$\begin{array}{l}\text { WATER } \\
\text { BEARING } \\
\text { ZONE }\end{array}$} & $\begin{array}{l}\text { CONCRETE (BCTIFF GROUT) } \\
\text { CLAY BLOCKS }\end{array}$ & 11.8 & $\begin{array}{l}629.0 \cdot 661.8 \\
661.8 \cdot 6749\end{array}$ \\
\hline & CONCRETE (BCTIFF GROUT) & 31.6 & $674.9 \cdot 707.7$ \\
\hline $\begin{array}{l}\text { SHAFT } \\
\text { BACKFILL }\end{array}$ & TO BE SPECIFIED & 11.8 & $707.7 \cdot 725.0$ \\
\hline \multirow{7}{*}{$\begin{array}{l}\text { UPPER } \\
\text { SHAFT }\end{array}$} & CONCRETE (BCT IFF GROUT) & 316 & $725.0 \cdot 757.8$ \\
\hline & CLAY BLOCKS & 11.8 & $757.8 \cdot 770.9$ \\
\hline & CONCRETE (BCTIFF GROUT) & 31.6 & $7709 \cdot 8037$ \\
\hline & CLAY BLOCKS & 11.8 & $803.7 \cdot 816.8$ \\
\hline & CONCRETE (BCTIFF GROUT) & 34.7 & $8168 \cdot 3496$ \\
\hline & CLAY BLOCKS & 15.0 & $849.6 \cdot 862.7$ \\
\hline & CONCRETE (BCTIF GROUT) & 31.6 & $862.7 \cdot 895.5$ \\
\hline \multirow[t]{8}{*}{$\begin{array}{l}\text { SHIAFT } \\
\text { BACKFILL }\end{array}$} & CRUSHED WIPP SALT & 11.8 & $895.5 \cdot 905.5$ \\
\hline & CONCRETE (BCTIF GROUT) & 31.6 & $905.5 \cdot 938.3$ \\
\hline & CLAY BLOCKS & 11.8 & $938.3-951.4$ \\
\hline & CRUSHED WIPP SALT (TAMPED) & 118 & $951.4 \cdot 9678$ \\
\hline & CLAY BLOCKS & 118 & $967.8 \cdot 980.9$ \\
\hline & CONCRETE (BCTIF GROUT) & 316 & $980.9-1013.7$ \\
\hline & CRUSHED WIPP SALT (TAMPED) & 11.8 & $1013.7 \cdot 1349.0$ \\
\hline & CONCRETE (BCTIF GROUT) & 31.6 & $1349.0 \cdot 13818$ \\
\hline \multirow{6}{*}{$\begin{array}{l}\text { LOWER } \\
\text { SHAFT }\end{array}$} & CLAY BLOCKS & 11.8 & $13818-13952$ \\
\hline & CRUSHED WIPP SALT TTAMPEDI & 118 & $13952 \cdot 14116$ \\
\hline & CLAY BLOCKS & 11.8 & $14116 \cdot 14247$ \\
\hline & CONCAETE IECTIF GAOUTI & $3 i 6$ & 1424714575 \\
\hline & CRUSHED $\because .: P P$ SALT ITAIIPEDI & $1: 8$ & $14575 \quad 105,50$ \\
\hline & $\therefore \cdots+\cdots+\cdots=r$ & $\cdots$ & $\ldots \quad-\cdots$ \\
\hline
\end{tabular}




\section{WASTE SHAFT}

\section{SHAFT SEAL SUMMARY}

\begin{tabular}{|c|c|c|c|}
\hline $\begin{array}{l}\text { SEAL } \\
\text { SYSTEM }\end{array}$ & MATEPIAL & $\begin{array}{l}\text { AXIAIUM } \\
\text { IETER (h.) }\end{array}$ & $\begin{array}{l}\text { SEAL INTERVAL } \\
\text { BELOW GFOUND } \\
\text { SURFACE (f.) }\end{array}$ \\
\hline $\begin{array}{l}\text { CAP COLLAF } \\
\text { BACKFILL. } \\
\text { AND PLUG }\end{array}$ & TO BE SPECIFIED & 23.0 & $0.0 \cdot 623.0$ \\
\hline \multirow{3}{*}{$\begin{array}{l}\text { WATER } \\
\text { BEARING } \\
\text { ZONE }\end{array}$} & CONCRETE (BCTIFF GROUT) & 42.7 & $623.0-6558$ \\
\hline & CLAY BLOCKS & 230 & $655.8 \cdot 668.9$ \\
\hline & CONCRETE ISCTIFF GROUTI & 42.7 & $668.9 \cdot 7017$ \\
\hline $\begin{array}{l}\text { SHAFT } \\
\text { BACKFILL }\end{array}$ & TO BE SPECIFIED & 23.0 & $7017 \cdot 718.0$ \\
\hline \multirow{7}{*}{$\begin{array}{l}\text { JPPER } \\
\text { SHAFT }\end{array}$} & CONCRETE (BCTIFF GROUT) & 427 & $718.0 \cdot 750.8$ \\
\hline & CLAY BLOCKS & 23.0 & $750.8 \cdot 763.9$ \\
\hline & CONCRETE (BCTIFF GROUT) & 42.7 & $763.9 \cdot 7967$ \\
\hline & CLAY BLOCKS & 23.0 & $796.7 \cdot 809.8$ \\
\hline & CONCRETE (BCTIFF GROUT) & 472 & $8098 \cdot 842.6$ \\
\hline & CLAY BLOCKS & 275 & $842.6 \cdot 885.7$ \\
\hline & CONCRETE IBCTIF GROUT; & 39.7 & $8857 \cdot 918.5$ \\
\hline \multirow[t]{8}{*}{$\begin{array}{l}\text { SHAFT } \\
\text { BACKFILL }\end{array}$} & CRUSHED WIPP SALT & 20.0 & $9135 \cdot 927.7$ \\
\hline & CONCRETE IBCTIF GROUTi & 39.7 & $9277 \cdot 960.5$ \\
\hline & CLAY BLOCKS & 20.0 & $9605 \cdot 973.6$ \\
\hline & CRUSHED WIPP SALT (TAMPED) & 200 & $973.6 \cdot 9900$ \\
\hline & CLAYY ELOCKS & 200 & $9900 \cdot 10031$ \\
\hline & CONCRETE (BCTIF GROUT) & $39 ?$ & $10031 \cdot 10359$ \\
\hline & CRUSHED WIPP SALT (TAMPED) & 20.0 & $1035.9-1356.0$ \\
\hline & CONCRETE (BCT IF GROUT) & 39.7 & $1356.0-1388.8$ \\
\hline \multirow{5}{*}{$\begin{array}{l}\text { LOWER } \\
\text { SHAFT }\end{array}$} & CLAY BLOCKS & 200 & $1388.8-14019$ \\
\hline & CRUSHED NIPP SALT (TAMPED) & 200 & $14019-14183$ \\
\hline & CLAY BLOCKS & 200 & $14183 \cdot 14314$ \\
\hline & CONCRETE (BCTIF GROUT) & 397 & $1.3141 .962 ?$ \\
\hline & 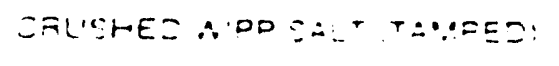 & $\therefore:$ & $\therefore \therefore \because 355=$ \\
\hline
\end{tabular}

\begin{tabular}{lr}
\multicolumn{1}{c}{ SEAL } \\
SYSTEM
\end{tabular}




\section{AIR INTAKE SHAFT}

\section{SHAFT SEAL SUMMARY}

\begin{tabular}{|c|c|c|c|}
\hline $\begin{array}{l}\text { SEAL } \\
\text { SYSTEM }\end{array}$ & MATERIAL & $\begin{array}{l}\text { XXIMUM } \\
\text { AETER (ft.) }\end{array}$ & $\begin{array}{l}\text { SEAL INTERVAL } \\
\text { BELOW GROUND } \\
\text { SURFACE (H) }\end{array}$ \\
\hline $\begin{array}{l}\text { CAP. COLLAR. } \\
\text { BACKFILL. } \\
\text { AND PLUG }\end{array}$ & TO BE SPECIFIED & 19.9 & $00 \cdot 529.0$ \\
\hline \multirow{3}{*}{$\begin{array}{l}\text { WATER } \\
\text { EEARING } \\
\text { ZONE }\end{array}$} & CONCRETE (BCTIFF GROUT) & 39.6 & 6290.6618 \\
\hline & CLAY BLOCKS & 199 & $661.867+9$ \\
\hline & CONCRETE (BCTIFF GROUT) & 39.6 & $67+9.7077$ \\
\hline $\begin{array}{l}\text { SHAFT } \\
\text { BACKFILL }\end{array}$ & TO BE SPECIFIED & 199 & $7077 \cdot 7250$ \\
\hline \multirow{7}{*}{$\begin{array}{l}\text { UPPER } \\
\text { SHAFT }\end{array}$} & \multirow{2}{*}{$\begin{array}{l}\text { CONCRETE (BCTIFF GROUT) } \\
\text { CLAY BLOCLSS }\end{array}$} & 39.6 & $7250 \cdot 7578$ \\
\hline & & 19.9 & $757.8 \cdot 7709$ \\
\hline & CONCRETE (BCTIFF GROUT) & 396 & $7709 \cdot 803.7$ \\
\hline & CLAY BLOCKS & 19.9 & 8037816.8 \\
\hline & CONCRETE (BCTIFF GROUT) & 453 & $8168 \quad 8496$ \\
\hline & CLAY BLOCKS & 256 & $8496 \cdot 8888$ \\
\hline & CONCRETE (BCTIF GROUT) & 40.0 & $8888 \cdot 9215$ \\
\hline $\begin{array}{l}\text { SHAFT } \\
\text { BACKFILL }\end{array}$ & CRUSHED WIPP SALT & 20.2 & 92169314 \\
\hline \multirow{12}{*}{$\begin{array}{l}\text { LOWJER } \\
\text { SHAFT }\end{array}$} & CONCRETE (BCTIF GROUT) & 400 & $9314 \cdot 964 ?$ \\
\hline & CLAY BLOCKS & 20.2 & $9642 \cdot 9774$ \\
\hline & CRUSHED WIPP SALT (TAMPED) & 202 & $9774 \cdot 993.8$ \\
\hline & CLAY BLOCHS & 20.2 & $9938 \cdot i 0053$ \\
\hline & CONCRETE (BCT IF GROUT) & 400 & $100691039 i$ \\
\hline & CRUSHED WIPP SALT (TAPAPED) & 20.2 & $10397 \cdot 1349.0$ \\
\hline & CONCRETE (BCTIF GROUT) & 400 & $13490 \cdot 13818$ \\
\hline & CLAY BLOCKS & 20.2 & $13818 \cdot: 395.2$ \\
\hline & CRUSHED WIPP SALT (TAMPED) & 202 & 1395214116 \\
\hline & CLAY BLOCKS & 20.2 & $14116 \cdot 1: 247$ \\
\hline & CONCRETE (BCTIF GROUT) & 400 & $14247 \cdot 1.45 i 5$ \\
\hline & 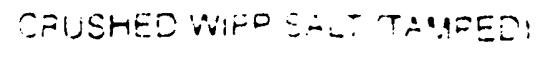 & 92 & $9=25.195=$ \\
\hline
\end{tabular}

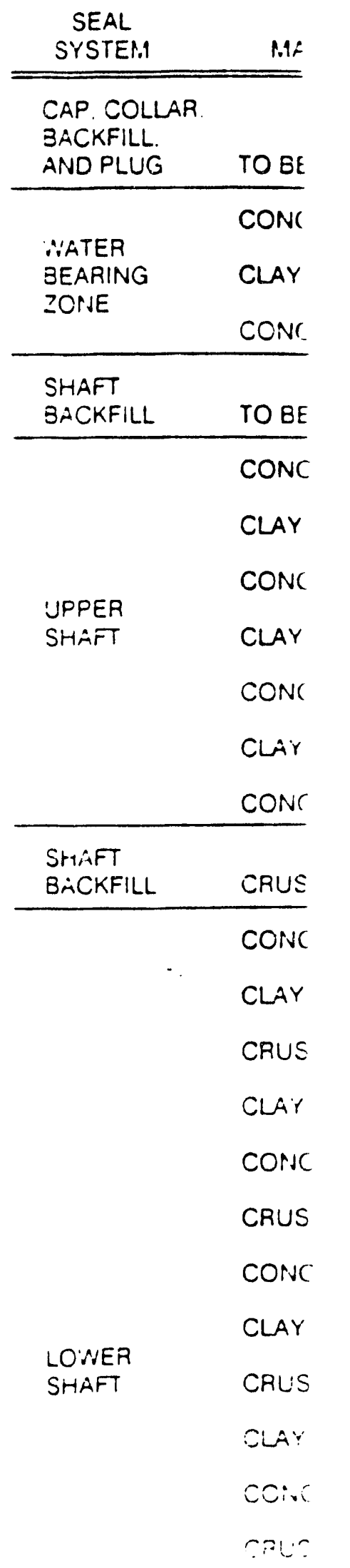




\section{EXHAUST SHAFT}

\section{SHAFT SEAL SUMMARY}

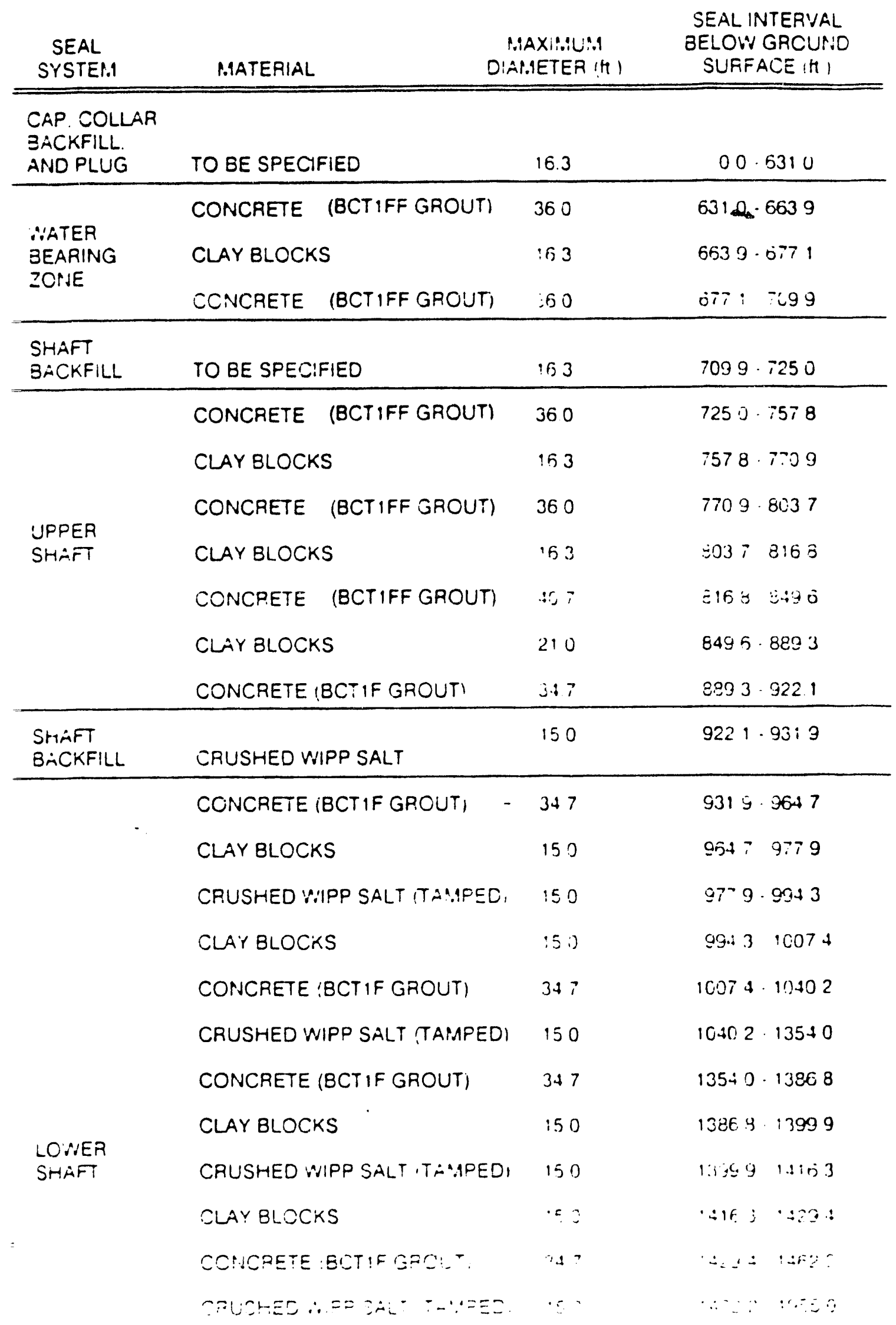


LINTERVAL JW GRCUR:D

F.FACE (H)

$\begin{array}{r}0.5310 \\ \hline 0.6639 \\ 9.0771 \\ \because .299 \\ \hline\end{array}$

$19 \cdot .250$

$50 \cdot 7578$

' $8 \cdot 7.709$

19.8037

$378: 66$

is 990

$15 \cdot 8803$

$33 \cdot 922.1$

$21.93: 3$

193647

1. 3779

$-9 \cdot \operatorname{gg} 3$

$: 3 \quad: 0074$

$74 \cdot 10402$

$02 \cdot 13540$

$40 \cdot 13868$

'E. $4 \quad 13999$

$\because 9 \quad 1.1 ; 33$

$\because: \because \because:$

$\because \therefore \quad \therefore \because \%$ 


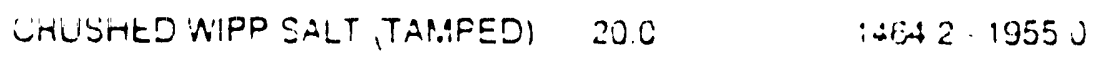

CONCRETE (BCTIF GROUT) $397 \quad 19550.19873$

$\begin{array}{lll}\text { CLAY BLOCKS } & 200 & 19878 \cdot 2000.9\end{array}$

CRUSHED WIPP SALT (TAMPED) $200 \quad 20009 \cdot 20173$

$\begin{array}{lll}\text { CLAY BLOCKS } & -20.0 & 20173 \cdot 20305\end{array}$

COICAETE ECTIF GROUT, 3'3

\begin{tabular}{lll} 
EFIF: & & \\
SACKFILL CRLSHED NIFF SALT & 230 & 20633.22060 \\
\hline
\end{tabular}

\section{EXISTING SHAFT DATA}

\begin{tabular}{|c|c|c|}
\hline $\begin{array}{l}\text { MAAUOR } \\
\text { FEMTURES }\end{array}$ & $\begin{array}{l}\text { LEVEL BELOW } \\
\text { SURFACE (it) }\end{array}$ & $\begin{array}{l}\text { ORIGINAL } \\
\text { EXCAVATION } \\
\text { DIAMETER (H }\end{array}$ \\
\hline SURFACE & 00 & $N, A$ \\
\hline BOTTOM.I OF COLLAR & 210 & 240 \\
\hline TOP OF MAGENTA & 597.0 & 23.0 \\
\hline 50:TTOM.1 OF MAGENTA & 6200 & 230 \\
\hline IOP OF CULEBRA & 7050 & 230 \\
\hline 30.TCH: OF CULEBRA & 7270 & 230 \\
\hline IOP OF KEY & 8390 & 230 \\
\hline FUSTLER SALADO CONTACT & 84.40 & 275 \\
\hline BOTTCH OF KEY & 3020 & 310 \\
\hline IUF OF JACA TRISTE & 13490 & 200 \\
\hline 3OTTC1.1 OF VACA TR!STE & 1356.0 & 200 \\
\hline CP OF STATION & 21470 & 230 \\
\hline STMTION & 21600 & $N, A$ \\
\hline TEP OF SUR.IP & 21600 & 230 \\
\hline EOTTOI.: OF SU:AP & $2: 860$ & 230 \\
\hline
\end{tabular}

SLAA
SUAAL
BOTTOH:
TOP OF
BOTTCH:
TOP OF
BOTTCH:
TOP OF
AUSTLE:
EOTTO::
TOP SF
EOTTLH:
TOP OF
STATIOH.


CLAY BLOCKS

CAUSHED WIPP SALT TTAMPEDI

202

2000920173

CLAY BLOCKS

202

2017320305

CONCRETE (BCT:F SFSUT,

400

$2030 \div 20533$

\section{EXISTING SHAFT DATA}

\begin{tabular}{|c|c|c|}
\hline $\begin{array}{l}\text { MA COR } \\
\text { FEATLFES }\end{array}$ & $\begin{array}{l}\text { LEVEL BELOW } \\
\text { SURFACE (H) }\end{array}$ & $\begin{array}{l}\text { ORIGINAL } \\
\text { EXCAVATION } \\
\text { DIAP.IETER :U }\end{array}$ \\
\hline & \multicolumn{2}{|c|}{ the } \\
\hline SLIRFACE & 00 & $N A$ \\
\hline BOTTOM.1 OF COLLAR & 16.0 & 199 \\
\hline TOP DF MAGENTA & 592.0 & 19.9 \\
\hline BOTTOP.1 OF MAGENTA & 627.0 & 199 \\
\hline TOP OF CULEBRA & 7100 & 19.9 \\
\hline SOTTOM OF CULEBRA & 7390 & 199 \\
\hline TCP OF KEY & 843.5 & 199 \\
\hline PUSTLER SALADO CONTACT & 8510 & 256 \\
\hline EОTTO:I OF KEY & 3065 & 312 \\
\hline TOP OF UACA TRISTE & 13480 & 202 \\
\hline EOTTORI OF VACA TRISTE & 13490 & 20.2 \\
\hline - OP OF STATION & 21350 & 202 \\
\hline STATION & 21500 & $N \cdot A$ \\
\hline
\end{tabular}

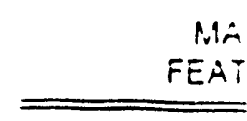

SURFAC:

BOTTOMA

TOP OF :

BOTTO:.:

TOF LF:

BOTTOR.:

TOP OF $r$

PUSTLEF

Gotrel.:

TUP:J.

sotici:?

POP DY:

STAROD.

\section{SNL}

\begin{tabular}{|c|c}
\hline ORG & DA \\
\hline$\therefore$ & $\ldots$ \\
\hline$\ldots \ldots$ &. \\
\hline$\therefore$ & \\
\hline$\therefore \cdots$ & 2 \\
\hline & \\
\hline & \\
\hline & \\
\hline
\end{tabular}


CONCRETE (BCTIF GF:SUT,

CLAY BLCCKS

150

CRUSHED WIPD SALT ITAMAPEDI

CLAY BLDCKS

i5j

$\therefore \div$

150

PJitis : $215 \%$ ?

\section{EXISTING SHAFT DATA}

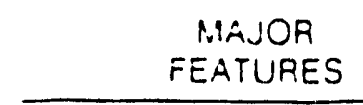

SURFACE

BOTTOM OF COLLAR

TOP OF MAGENTA

BOTTOM.: OF MAGENTA

IOF OF CULEBRA

BOTTOI.1 OF CULEERA

TOP OF KEY

=USTLEA SALADO CCRTAACT

SOTTON.1 OF NEY

TUP GF AACA TRISTE

SOTTOA OF VACA TFISTE

POP UF STATION

STAIOON
LEVEL BELOW SURFACE (I)

00

10.0

0030

6270

i1: 0

7350

8.450

3510

wi:

$: 5: 50$

$1.57 \%$

21470

45.
OEIGINA-

EXCAVATIO::

DIARETEA
NOIES

1. TABLES THAT GENIERATED, THE FOLLOW:
A. WASTES: (BECHTE: DRAWINC
B. AIR INTAH BECHTE: DRAWINC
C. EXHAUSI BECHTE: DRAWING
D. EXPLOR, (BECHTEI DRAWING

2. ALL STRATIGF ACCORDING T ENERGY, OCT REPORT, DOE FRANCISCO.

* EXPLORATCF CONSTRUCTIK

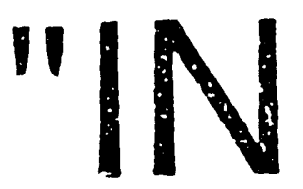

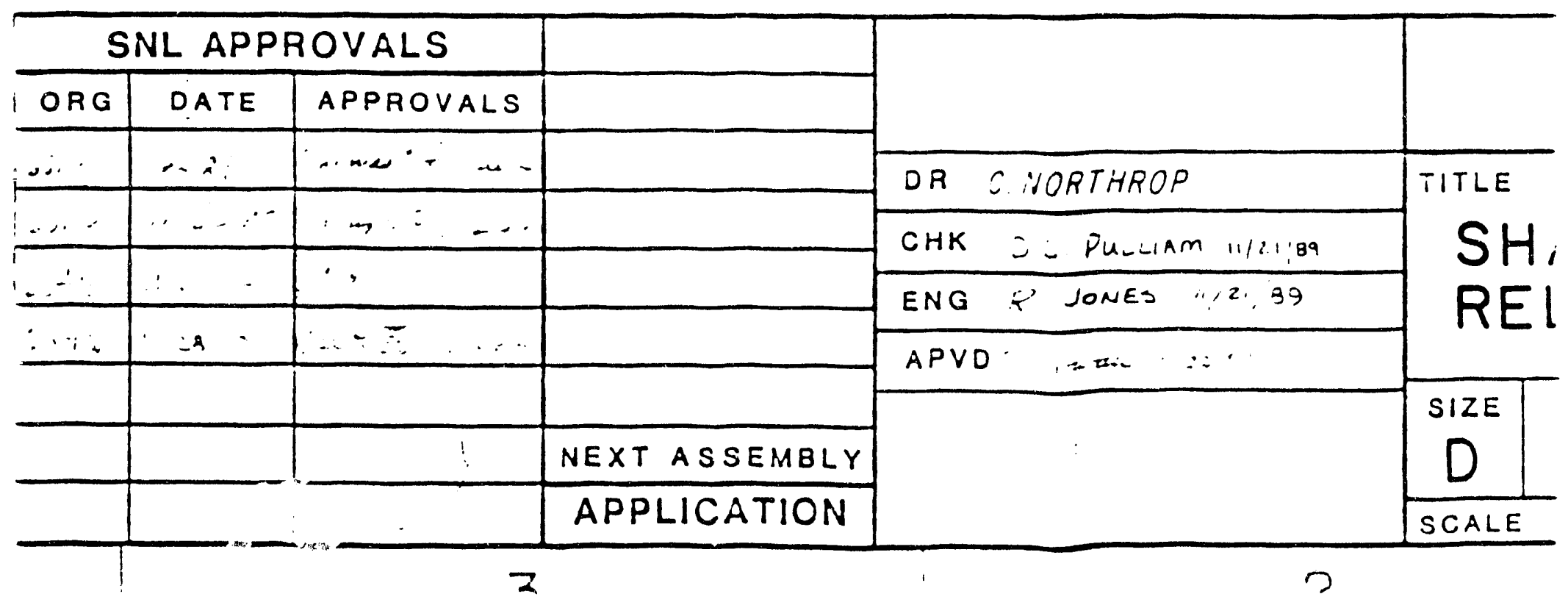


1. TABLES THAT PRESENT EXISTING SHAFT DATA ARE GENIERATED ACCORDING TO INFORMATION OBTAINED FROM THE FOLLOWING DRAWING SETS.

A WASTE SHAFT US DOE WIPP DRAWING 31-R-013-OID BECHTEL. SAN FRANCISCO. CA) AND US DOE iWIPP DRAWING 31-R-002-OID (WESTINGHOUSE. CARLSBAD. NM).

B. AIR INTAKE SHAFT US DOE, WIPP DRAWING 33-R-001-34A BECHTEL. SAN FRANCISCO. CA) AND US DOE:WIPP DRAWING 33-R-029-34A (BECHTEL. SAN FRANCISCO. CA).

C. EXHAUST SHAFT. US DOE.'WIPP DRAWING 35-R-001-OID (BECHTEL. SAN FRANCISCONCA) AND US DOE; WIPP DRAWING 35-R-002-OID (BECHTEL. SAN FRANCISCO. CA).

A

D. EXPLORATORY SHAFT US DOE, WIPP DRAWING 37-R.010 (BECHTEL. SAN FRANCISCO. CA) AND US DOE/WIPP DRAWING 37-R-012 (BECHTEL. SAN FRANCISCO. CA).

2. ALL STRATIGRAPHIC FEATURES ARE REPRESENTED ACCORDING TO DATA COMPILED FROM U.S. DEPARTMIENT OF ENERGY, OCTOBER 1986. WIPP DESIGN VALIDATION FINAL REPORT, DOE-WIPP-86-010. BECHTEL NATIONAL INC. SAN FRANCISCO. CA.

-EXPLORATORY SHAFT HAS SINCE BEEN CHANGED TO CONSTRUCTION \& SALT HANDLING SHAFT

\section{川}

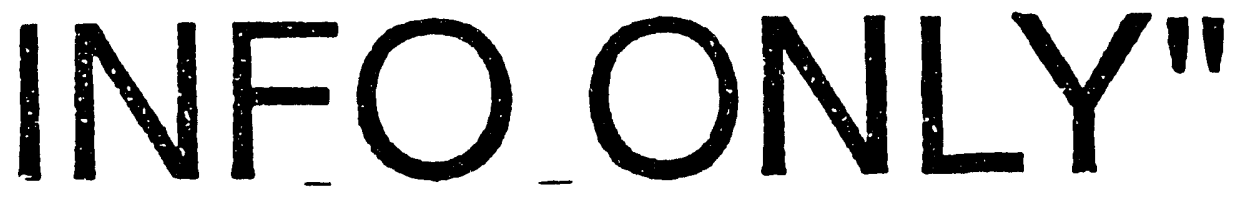

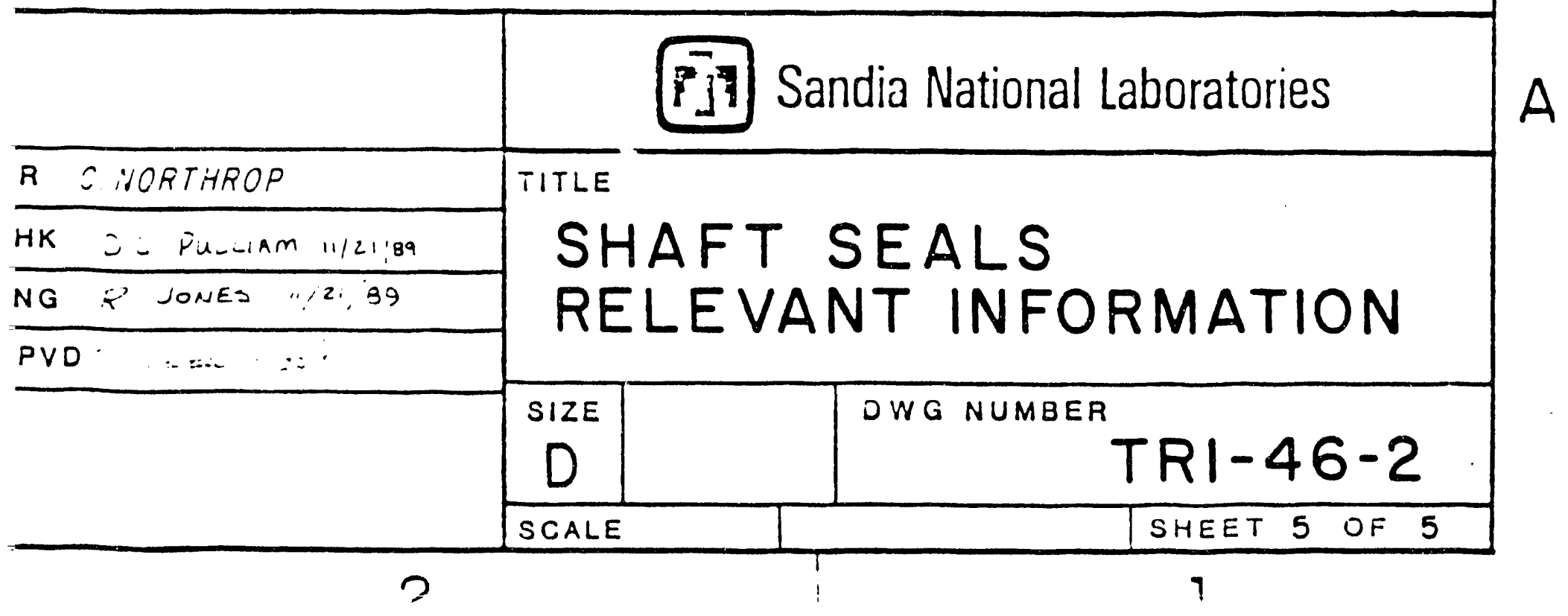




\section{Drift and Panel Seals General Arrangement TRI-46-3}

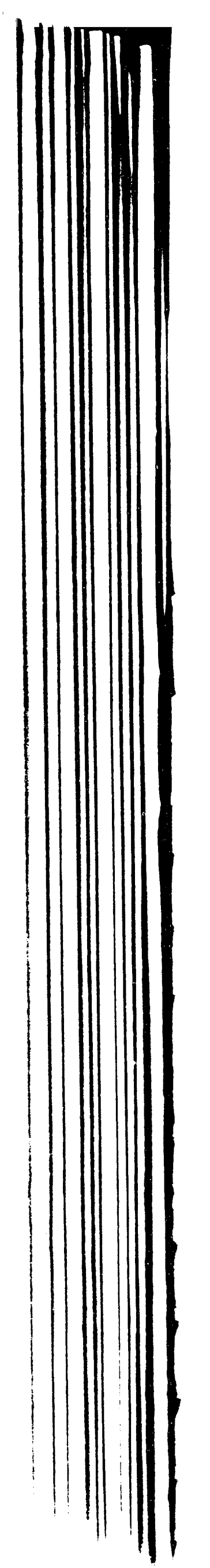




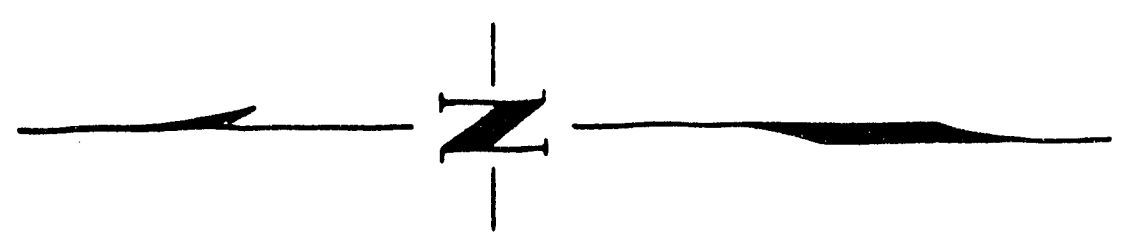

D

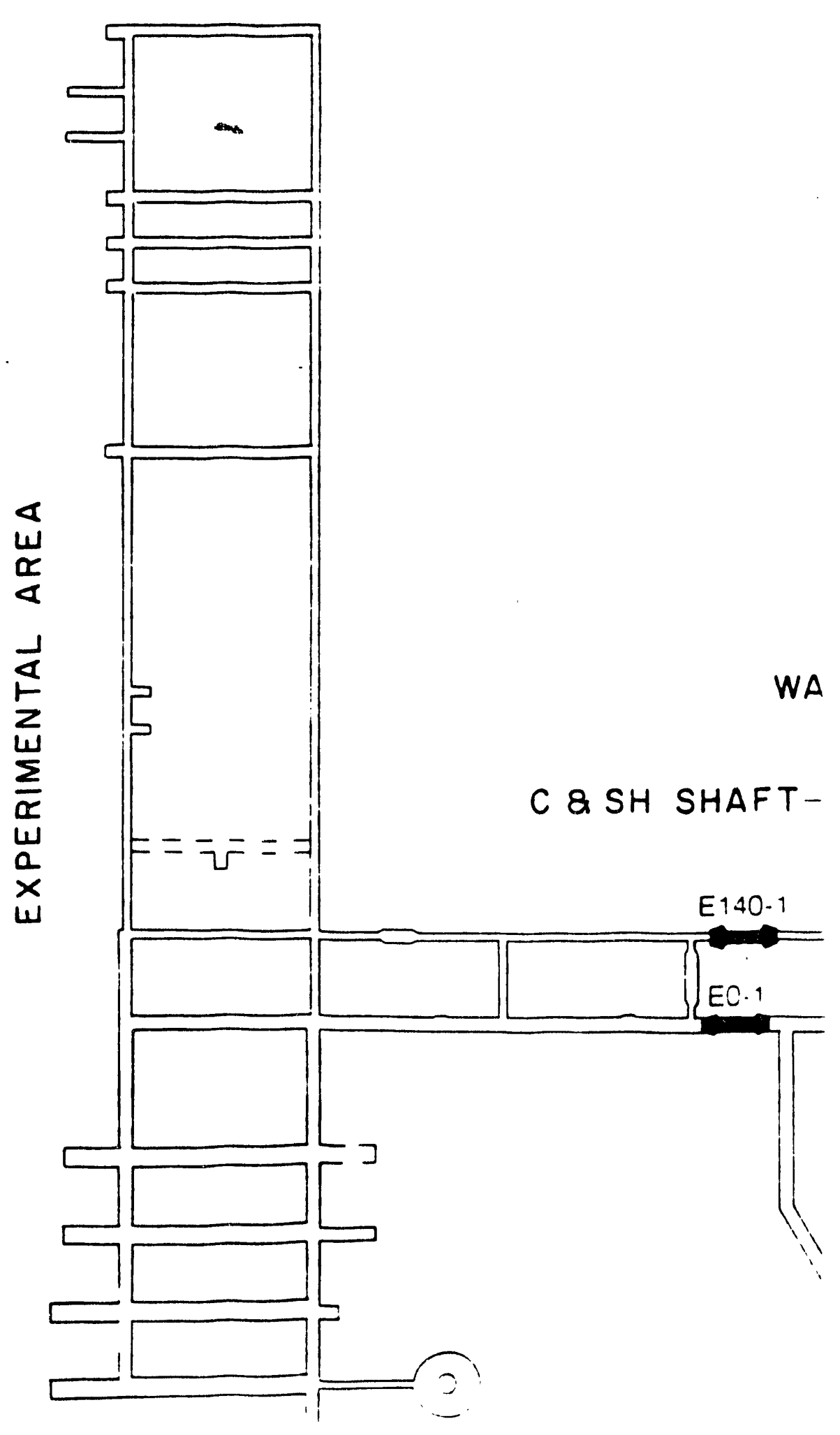



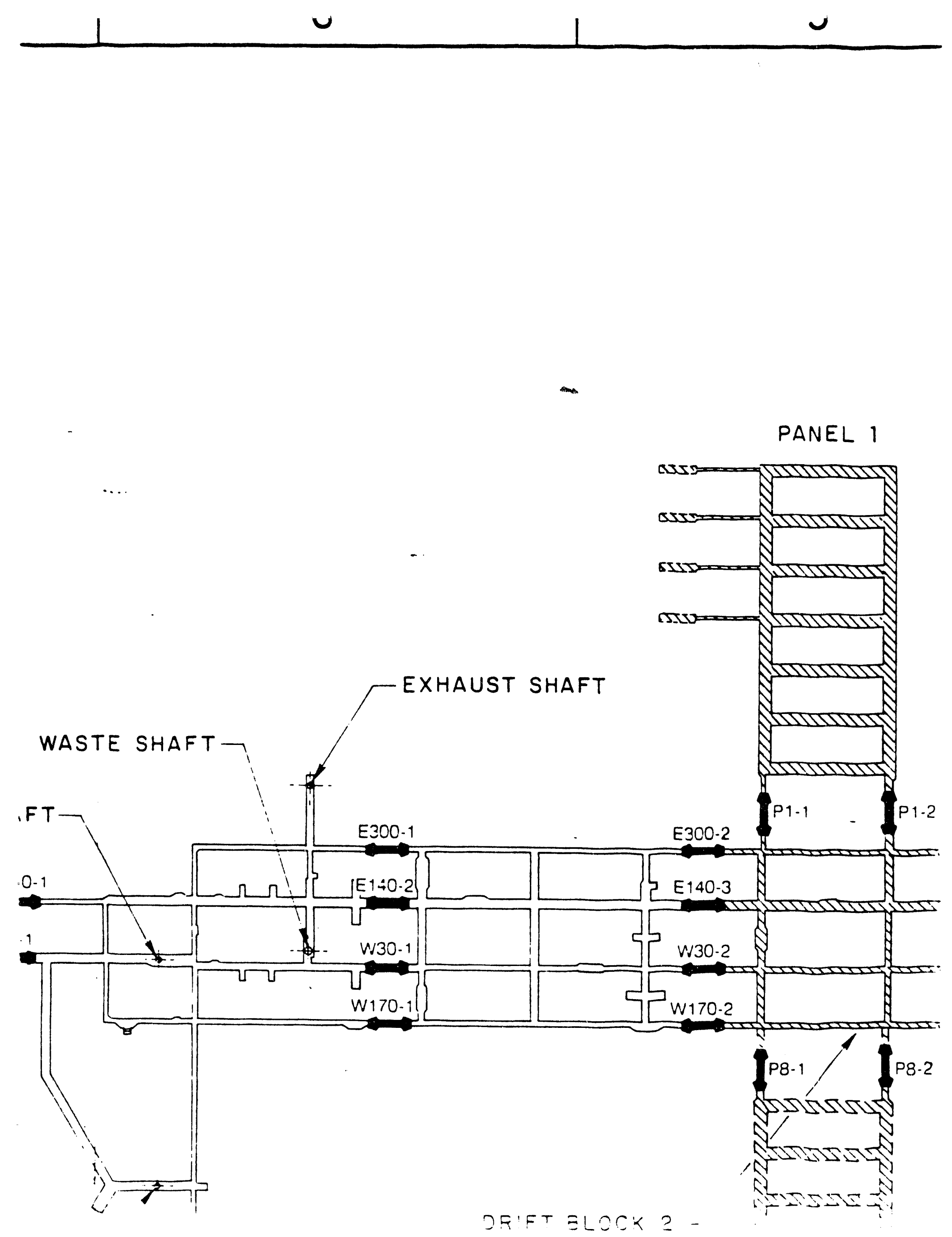


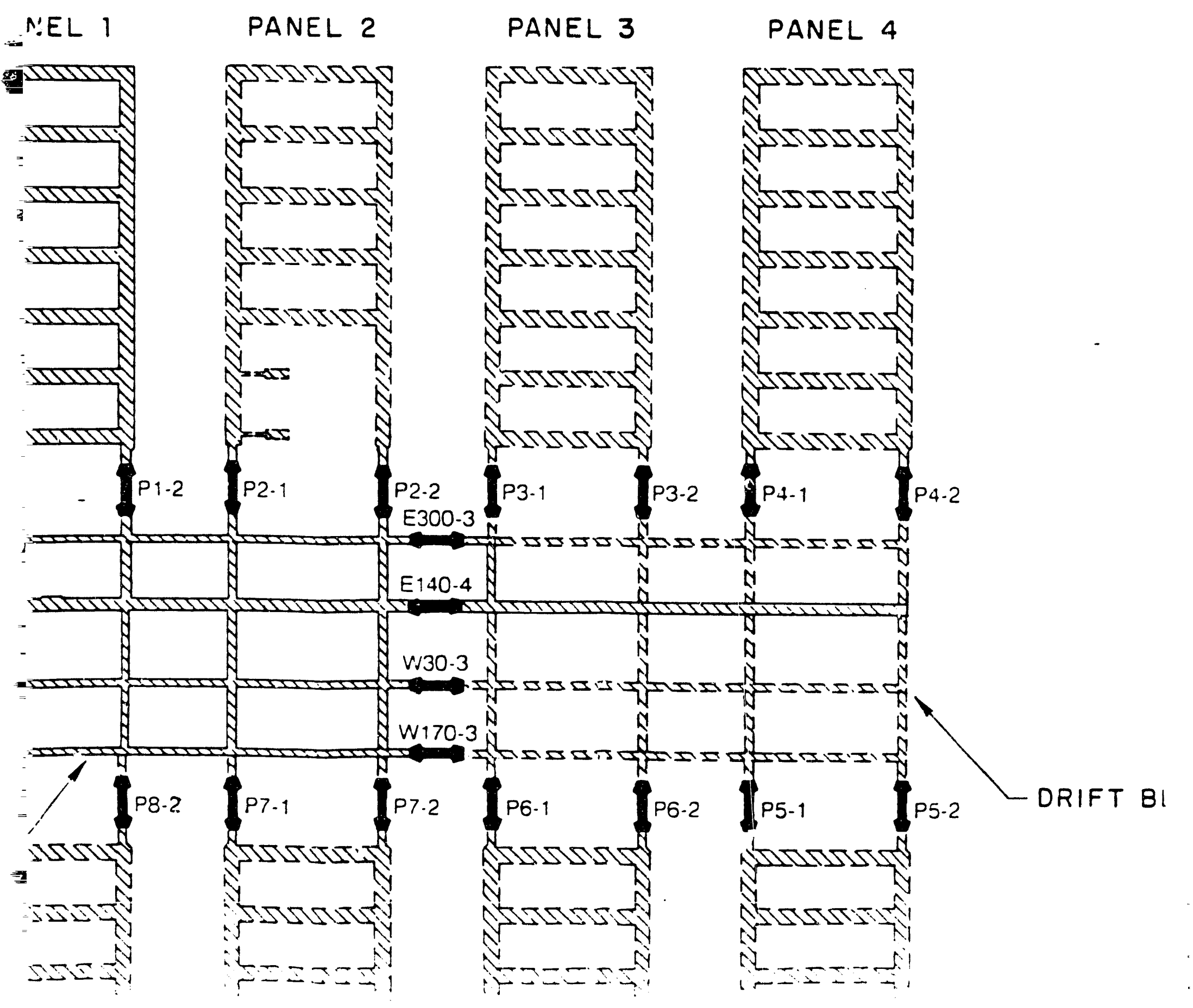




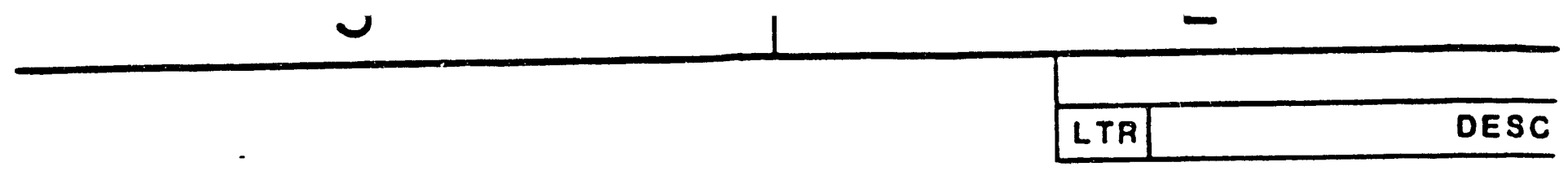

DRIFT DIMENSIONS AT PROPOSED SEAL LOCATIONS

$\begin{array}{lll}\text { SEAL } & \text { NOMINAL } & \text { NOMINAL } \\ \text { WUMBER } & \text { WIDTH (ft) } & \text { HEIGHT (ft) }\end{array}$

\begin{tabular}{|c|c|c|c|}
\hline & $\begin{array}{l}\text { E300-1 } \\
\text { E300-2 } \\
\text { E300-3 }\end{array}$ & $\begin{array}{l}14 \\
14 \\
14\end{array}$ & $\begin{array}{l}12 \\
12 \\
12\end{array}$ \\
\hline & $\begin{array}{l}\text { E140.1 } \\
\text { E140.2 } \\
\text { E140.3 } \\
\text { E140.4 }\end{array}$ & $\begin{array}{l}14 \\
25 \\
25 \\
25\end{array}$ & $\begin{array}{l}18 \\
12 \\
12 \\
12\end{array}$ \\
\hline $\begin{array}{l}\text { DRIFT } \\
\text { SEALS }\end{array}$ & EO-1 & 25 & 12 \\
\hline & $\begin{array}{l}\text { W30-1 } \\
\text { W30-2 } \\
\text { W30-3 }\end{array}$ & $\begin{array}{l}20 \\
14 \\
14\end{array}$ & $\begin{array}{l}12 \\
12 \\
12\end{array}$ \\
\hline & $\begin{array}{l}W 170.1 \\
W 170.2 \\
W 170.3\end{array}$ & $\begin{array}{l}14 \\
14 \\
14\end{array}$ & $\begin{array}{l}12 \\
12 \\
12\end{array}$ \\
\hline
\end{tabular}

DRIFT BLOCK 1

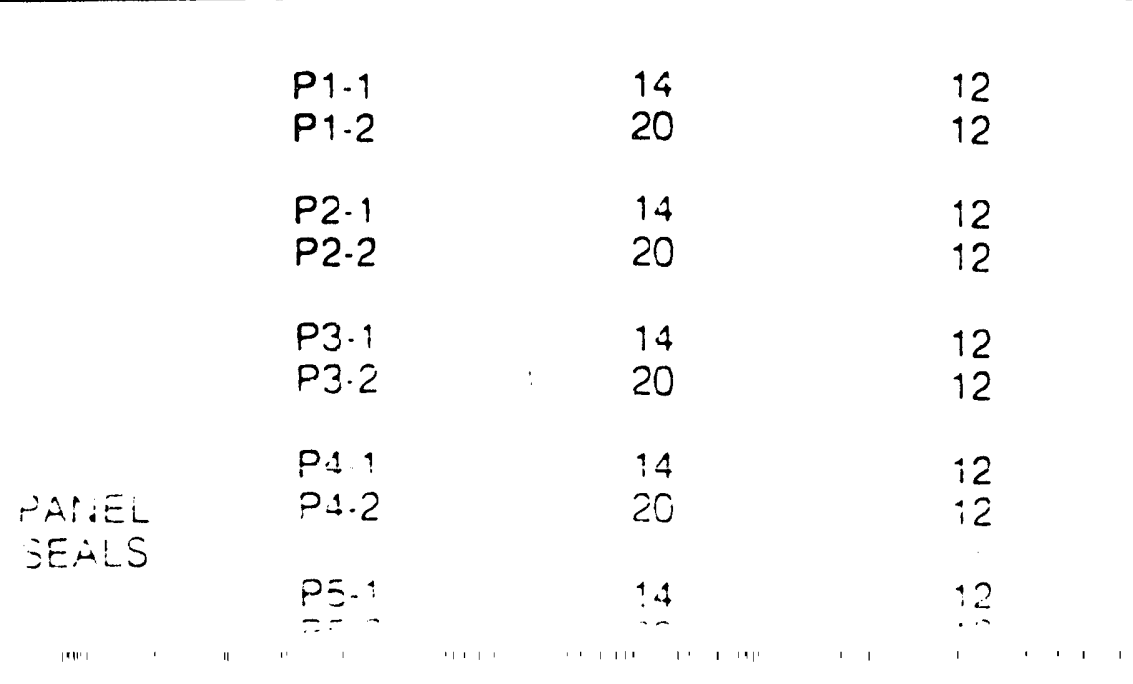


IEAL NOMINAL NOMINAL *SECTION

MBER WIDTH (tt) HEIGHT (ft) TYPE

$\begin{array}{llll}300-1 & 14 & 12 & \text { C.C } \\ 300-2 & 14 & 12 & \text { C.C } \\ 300-3 & 14 & 12 & \text { C.C } \\ 140-1 & 14 & 18 & \text { B.B } \\ 140-2 & 25 & 12 & \text { A.A } \\ 140-3 & 25 & 12 & \text { A-A } \\ 140-4 & 25 & 12 & \text { A-A } \\ 0-1 & 25 & 12 & \text { A-A } \\ 130-1 & 20 & 12 & \text { D-D } \\ 130-2 & 14 & 12 & \text { C.C } \\ 130-3 & 14 & 12 & \text { C.C } \\ 170-1 & 14 & 12 & \text { C.C } \\ 170-2 & 14 & 12 & \text { C.C } \\ 170-3 & 14 & 12 & \text { C.C }\end{array}$

$1-1$

$1-2$

14

$2-1$

2-2

$3-1$
3 $3-2$

3. 1

3.2

20

14

20

14

20

14
20

12

12

C.C

D.D

12

12

C.C

D.D

12

12

C.C

D.D

12
12

C.C

D.D 


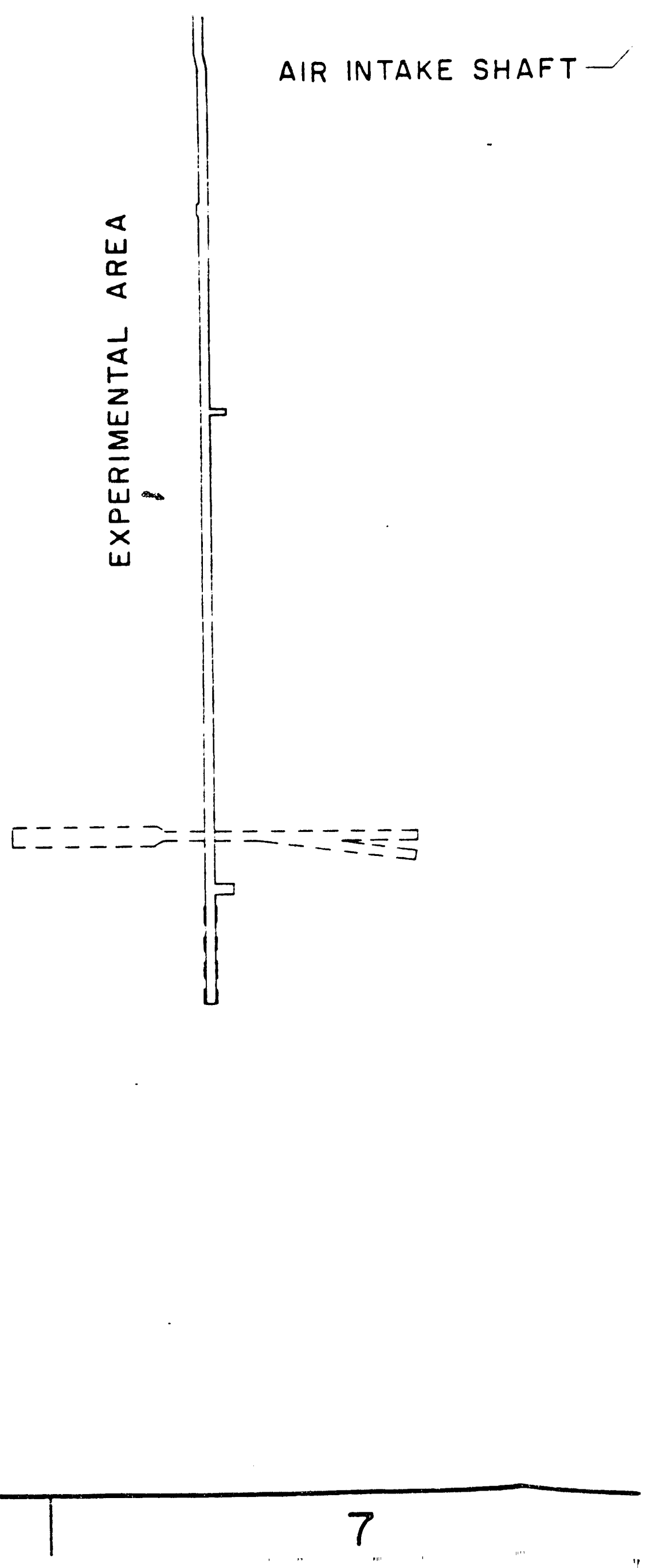




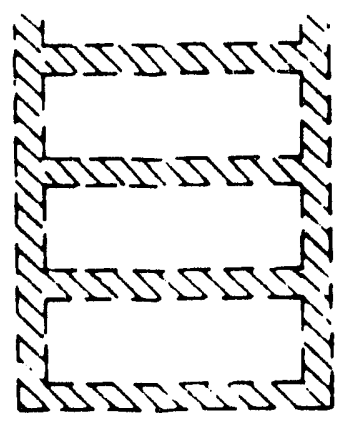

PANEL 8

LEGEND

$\begin{array}{ll}\square & \text { EXISTING EXCAVATION } \\ \overline{-}=- & \text { PLANNED EXCAVATION } \\ \text { DRIFT SEALS } \\ \text { WASTE DISPOSAL AREAS }\end{array}$

NOTES

1. DRIFT BLOCK $1 \mathrm{CC}$ BOUNDED BY THE P5-2. P5-1, P6-2, P6

2. DRIFT BLOCK $2 \mathrm{CO}$ BOUNDED BY THE P7-2. P7-1. P8-2, PQ

3. IN EACH WASTE DI BACKFILLING. AND PROCEEDING WITH THE WASTE DISPO BLOCK 1, AND DRII AREA TO BE COMF CONTINUING IN NI AFTER THE EIGHT WILL BE COMPLET

THE SEALS WILL B, DISPOSAL AREA AI SEALS P1-1 AND $P$

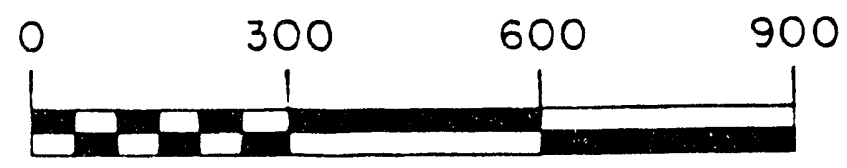
EMPLACEMENT AN E300-3, E140-4, W3 WASTE EMPLACEN

SCALE IN FEET 


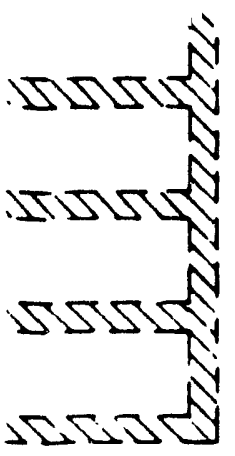

INEL 8

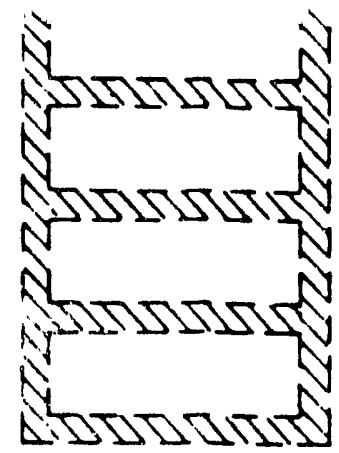

PANEL 7

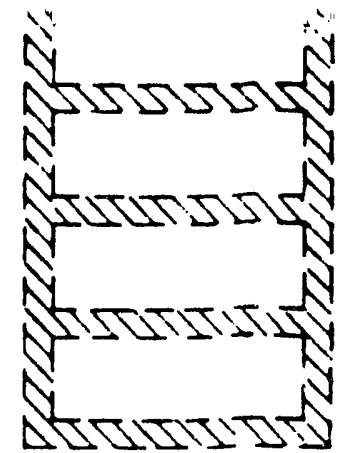

PANEL 6

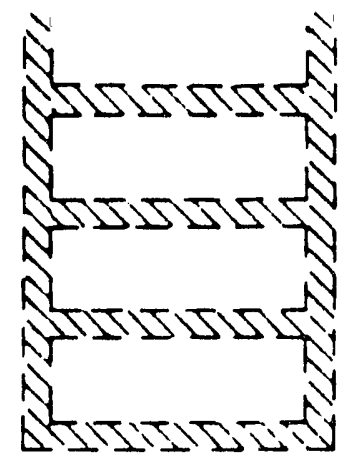

PANEL 5

"

IRIFT BLOCK 1 CONSISTS OF THE INTERSECTING DRIFTS :OUNDED BY THE FOLLOWING SEALS: P3-1, P3-2, P4-1, P4-2. 5-2. P5-1, P6-2, P6-1, W170-3, W30-3, E140-4, AND E300-3.

IRIFT BLOCK 2 CONSISTS OF THE INTERSECTING DRIFTS .OUNDED BY THE FOLLOWING SEALS: P1-1, P1-2. P2-1, P2-2, 7-2. P7-1, P8-2, P8-1. W170-2, W30-2, E140-3, AND E300-2.

$\checkmark$ EACH WASTE DISPOSAL AREA, WASTE EMPLACEMENT. .ACKFILLING. AND SEALING WILL BE COMPLETED BEFORE ROCEEDING WITH THOSE OPERATIONS IN THE NEXT AREA. HE WASTE DISPOSAL AREAS ARE PANELS 1 THROUGH 8, DRIFT LOCK 1, AND DRIFT BLOCK 2. PANEL 1 VIILL BE THE FIRST REA TO BE COMPLETED. FOLLOWED BY PANEL 2. AND ONTINUING IN NUMERICAL SEQUENCE THROUGH PANEL 8. FTER THE EIGHT PANELS ARE FINISHED. THE DRIFT BLOCKS IILL BE COMPLETED; FIRST BLOCK 1 FOLLOWED BY BLOCK 2.

HE SEALS WILL BE CONSTRUCTED BETWEEN EACH WASTE ISPOSAL AREA AND OPEN ACCESS DRIFTS. FOR EXAMPLE. EALS P1-1 AND P1-2 WILL BE BUILT FOLLOWING WASTE MPLACEMENT AND BACKFILLING IN PANEL 1, AND SEALS 300-3, E140-4, W30-3, AND W170-3 WILL BE BUILT FOLLOWING 'ASTE EMPLACEMENT AND BACKFILLING IN DRIFT BLOCK 1.

SNL APPROVALS

\begin{tabular}{|c|c|c|}
\hline ORG & DATE & APPROV \\
\hline$\therefore$ & , i & 'R' \\
\hline$\therefore \cdot$ & 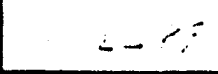 & $\because-\operatorname{sen}:$ \\
\hline$\therefore 3 \div$ & $11-B E-E S$ & 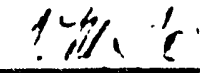 \\
\hline 8345 & $11 /=3 / 9 \pi$ & Jse QT, \\
\hline & & \\
\hline & & \\
\hline & & \\
\hline & & \\
\hline
\end{tabular}




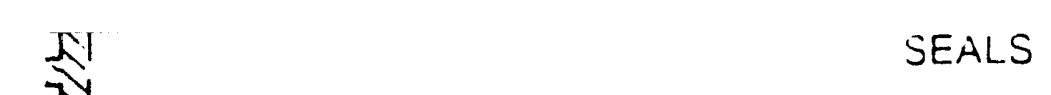

$\begin{array}{lll}\text { P5-1 } & 14 & 12 \\ \text { P5-2 } & 20 & 12 \\ \text { P5. } 1 & 14 & 12 \\ \text { P5-2 } & 20 & 12 \\ \text { P7. } & 14 & 12 \\ \text { P7.2 } & 20 & 12 \\ \text { P8.1 } & 14 & 12 \\ \text { P8.2 } & 20 & 12\end{array}$

j

* Refer to tri-46-3. 2 of 2 for section detalls

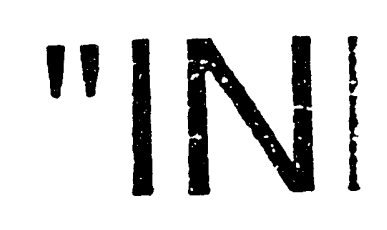

\begin{tabular}{|c|c|c|c|c|c|}
\hline \multicolumn{3}{|c|}{ SNL APPROVALS } & & & \\
\hline \multirow[t]{2}{*}{$\mathbf{R G}$} & DATE & APPROVALS & & & \\
\hline & $\therefore ;=$ & 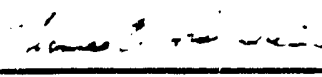 & & DR W. DUMAS & \multirow{4}{*}{$\begin{array}{l}\text { TItLE } \\
\text { DRI } \\
\text { GFN }\end{array}$} \\
\hline$\dot{-}$ & $\ldots$ & $\therefore \ldots=\ldots$ & & CHK כL. PULLIAM $\because 1,-1,89$ & \\
\hline$\therefore t=$ & $11-E-E:$ & 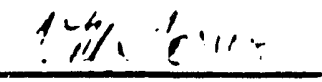 & & ENG $₹$-ONES $\| 2189$ & \\
\hline \multirow[t]{4}{*}{ : } & $1: /=3 / 3 ?$ & Hese QT,CLC..om & & APVD, & \\
\hline & & & & & SIZE \\
\hline & & & NEXT ASSEMBLY & & $D$ \\
\hline & & & APPLICATION & & SCALE 1"= \\
\hline
\end{tabular}




\begin{tabular}{|c|c|c|}
\hline 5.2 & 20 & 12 \\
\hline $\begin{array}{l}6.1 \\
5.2\end{array}$ & $\begin{array}{l}14 \\
20\end{array}$ & $\begin{array}{l}12 \\
12\end{array}$ \\
\hline $\begin{array}{l}7 . ; \\
7.2\end{array}$ & $\begin{array}{l}14 \\
20\end{array}$ & $\begin{array}{l}12 \\
12\end{array}$ \\
\hline $3-1$ & $\begin{array}{l}14 \\
20\end{array}$ & $\begin{array}{l}12 \\
12\end{array}$ \\
\hline
\end{tabular}

71.45-3. 2 OF 2 FOR SECTION DETAILS

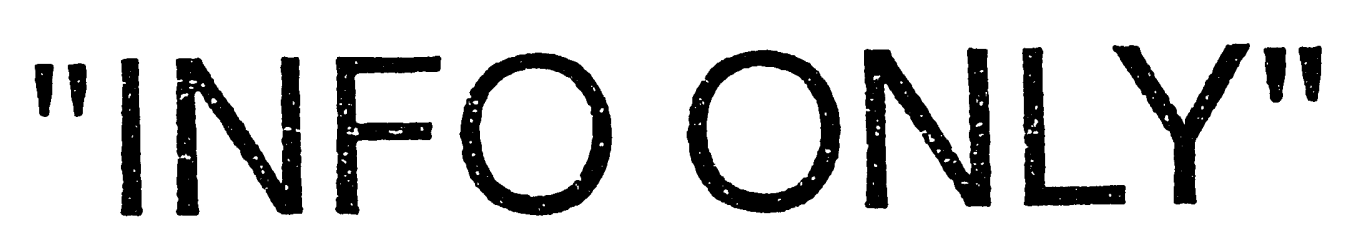

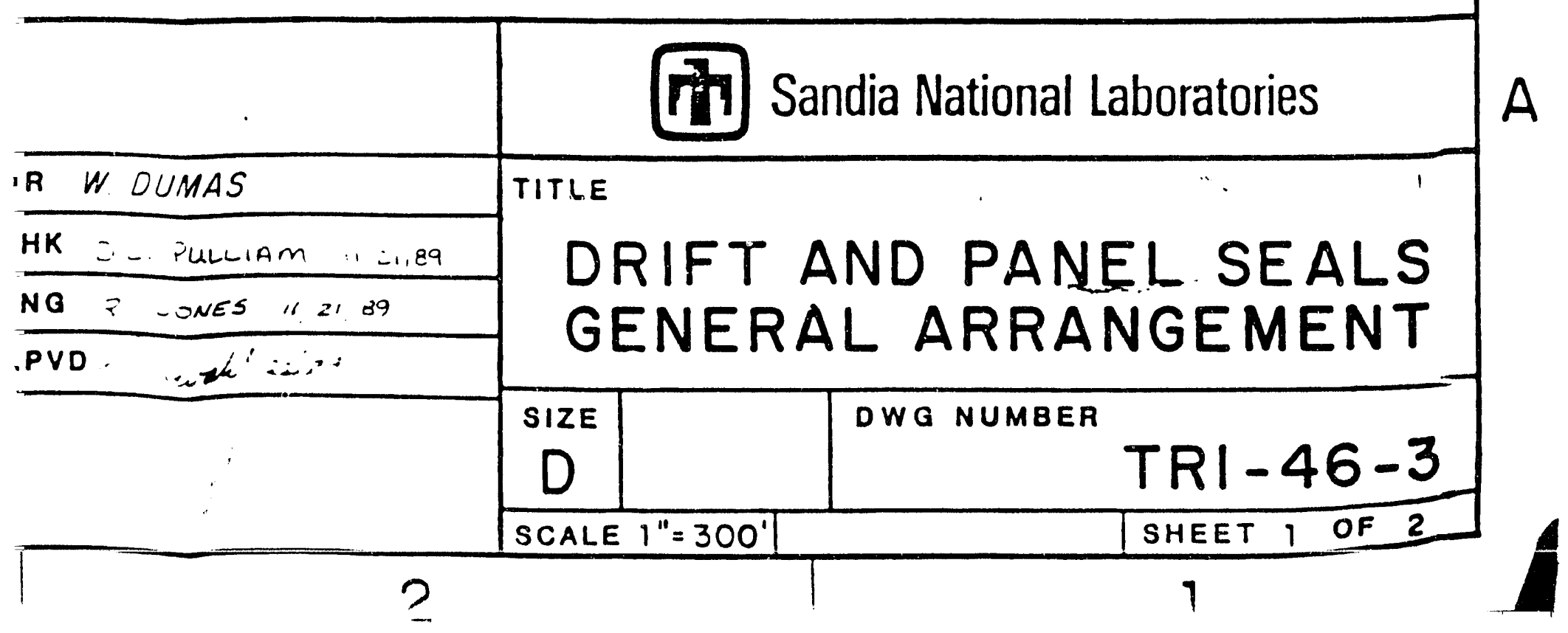




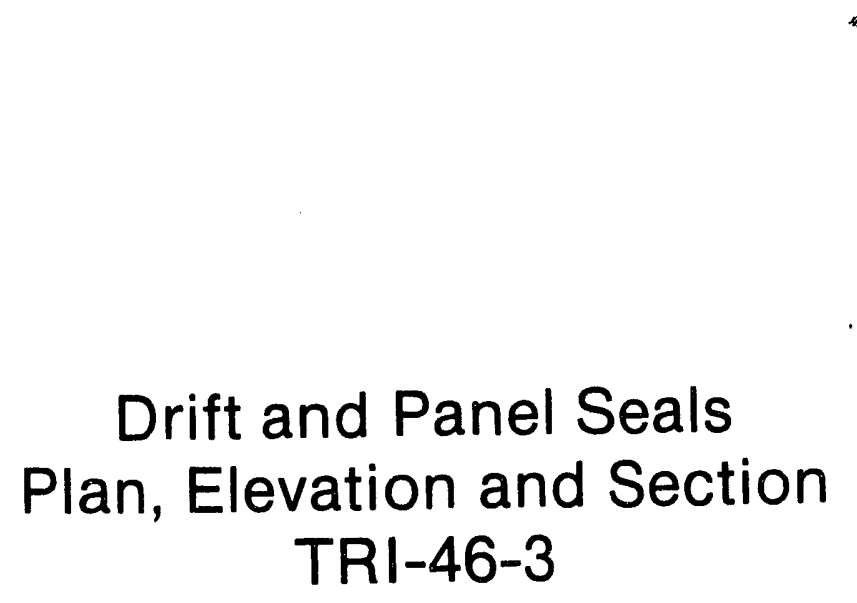



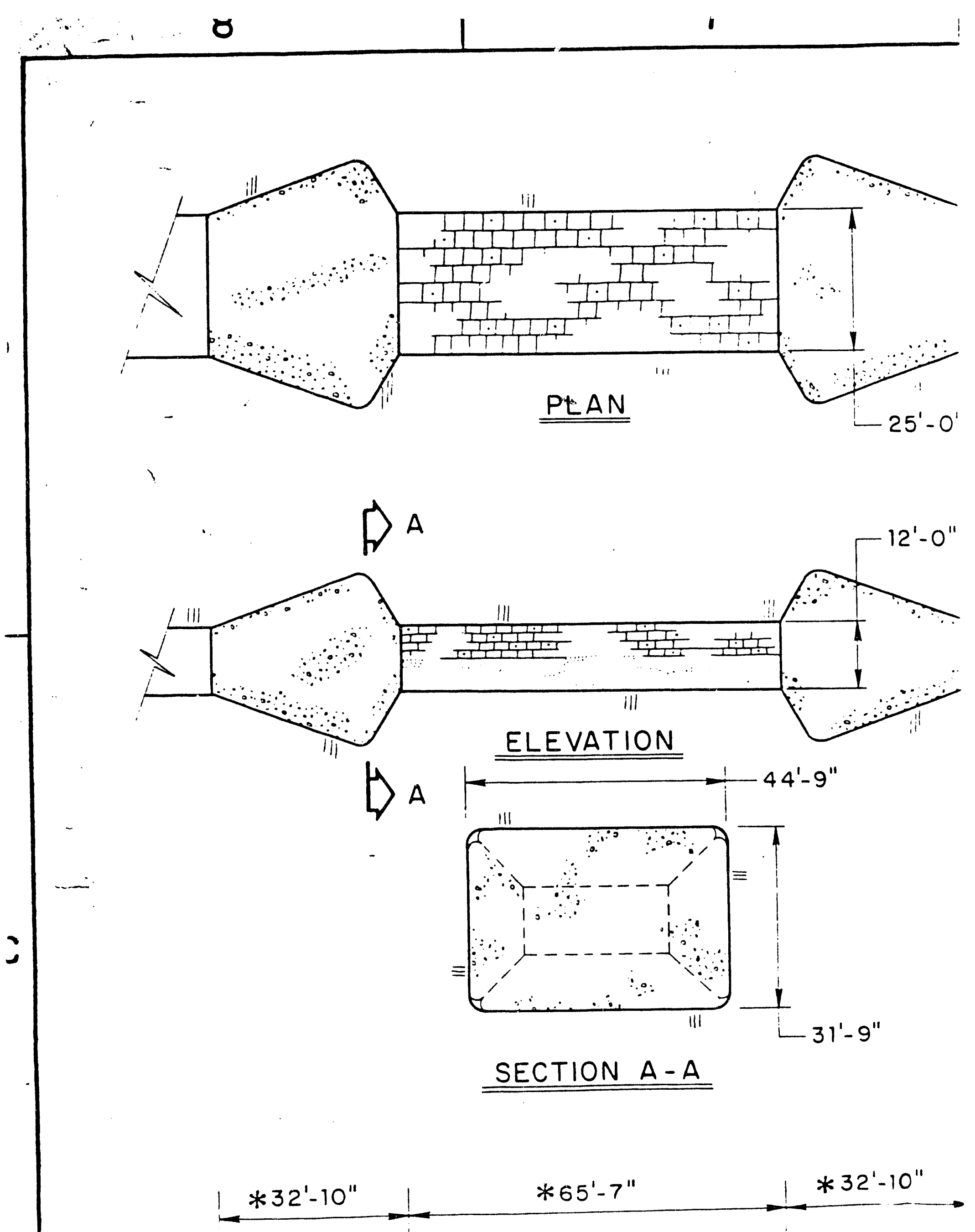

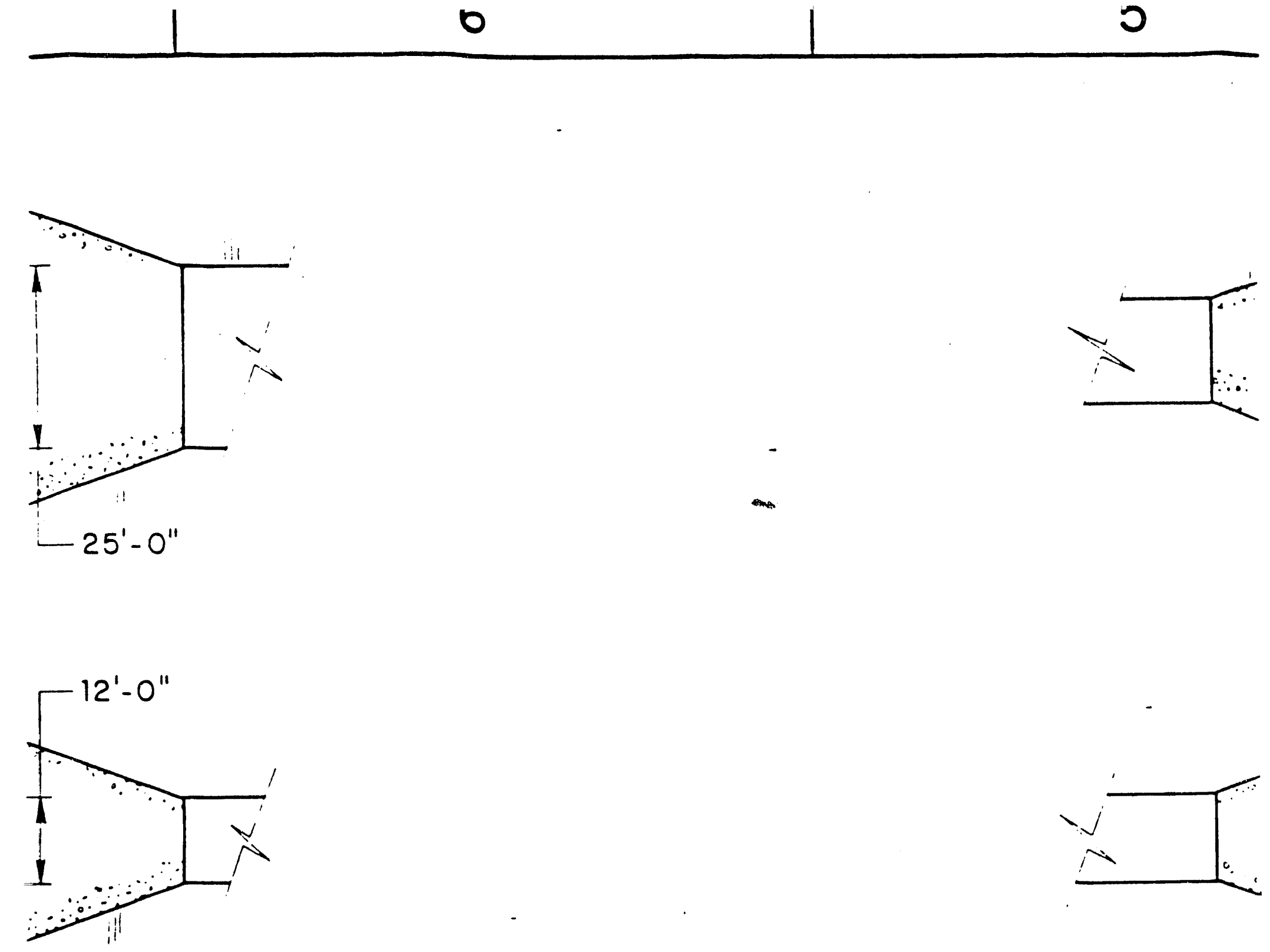

PRECONSOLIDATED SALT, (FOR DESCRIPTION OF MATERIAL SEE NOTE A4 ON DRAWING NUMBER TR:

.91

$2^{\prime}-10^{\prime \prime}$ 


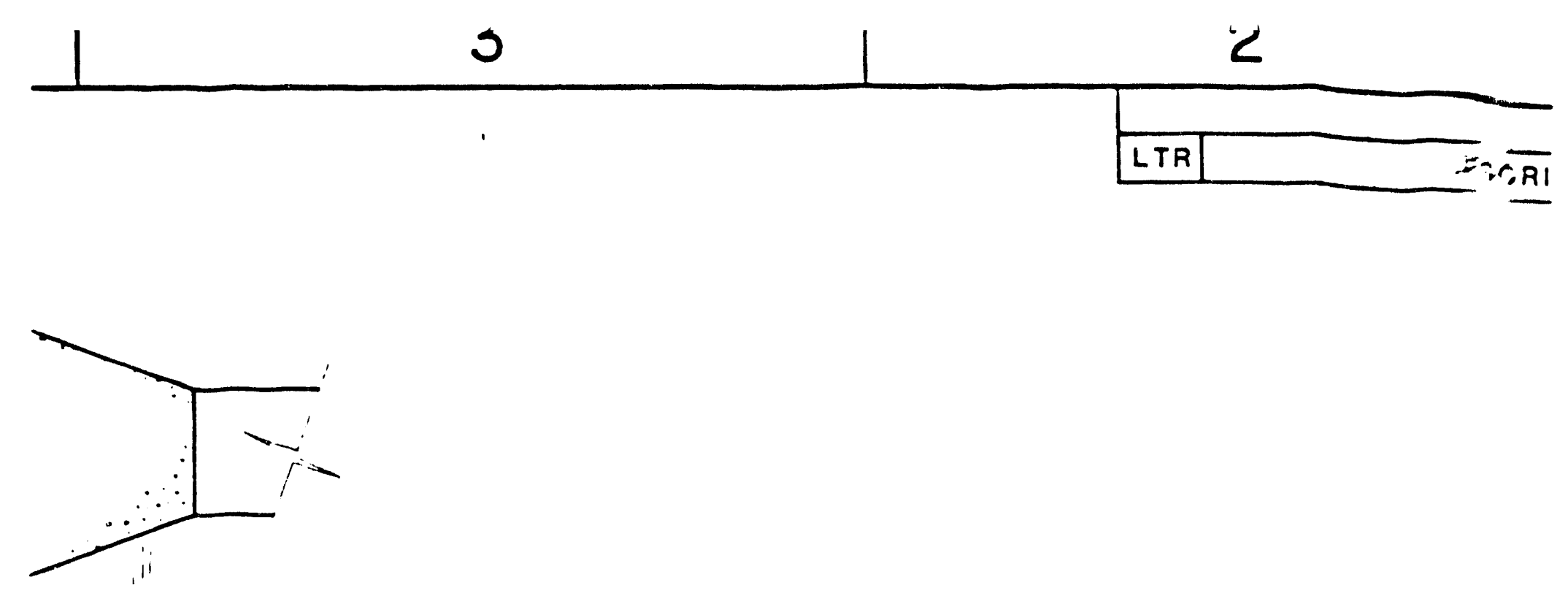

$-14^{\prime}-0^{\prime \prime}$

.m.

$-12^{\prime}-0^{\prime \prime}$

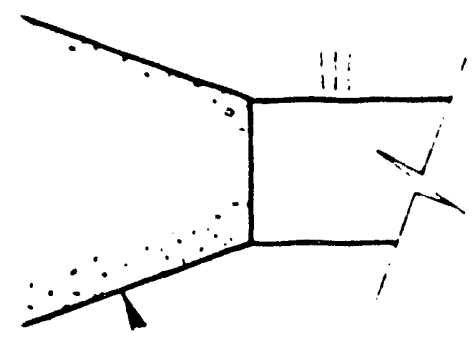

LCONCRETE, TYPICAL

(FOR DESCRIPTION OF MATERIAL SEE

NOTE A1 ON DRAWING NUMBER TRI-46-5;

\section{NOTES}

1. MMLHITE: . . O TH: UPPE: - C W Wikipds TARIPIRUS 


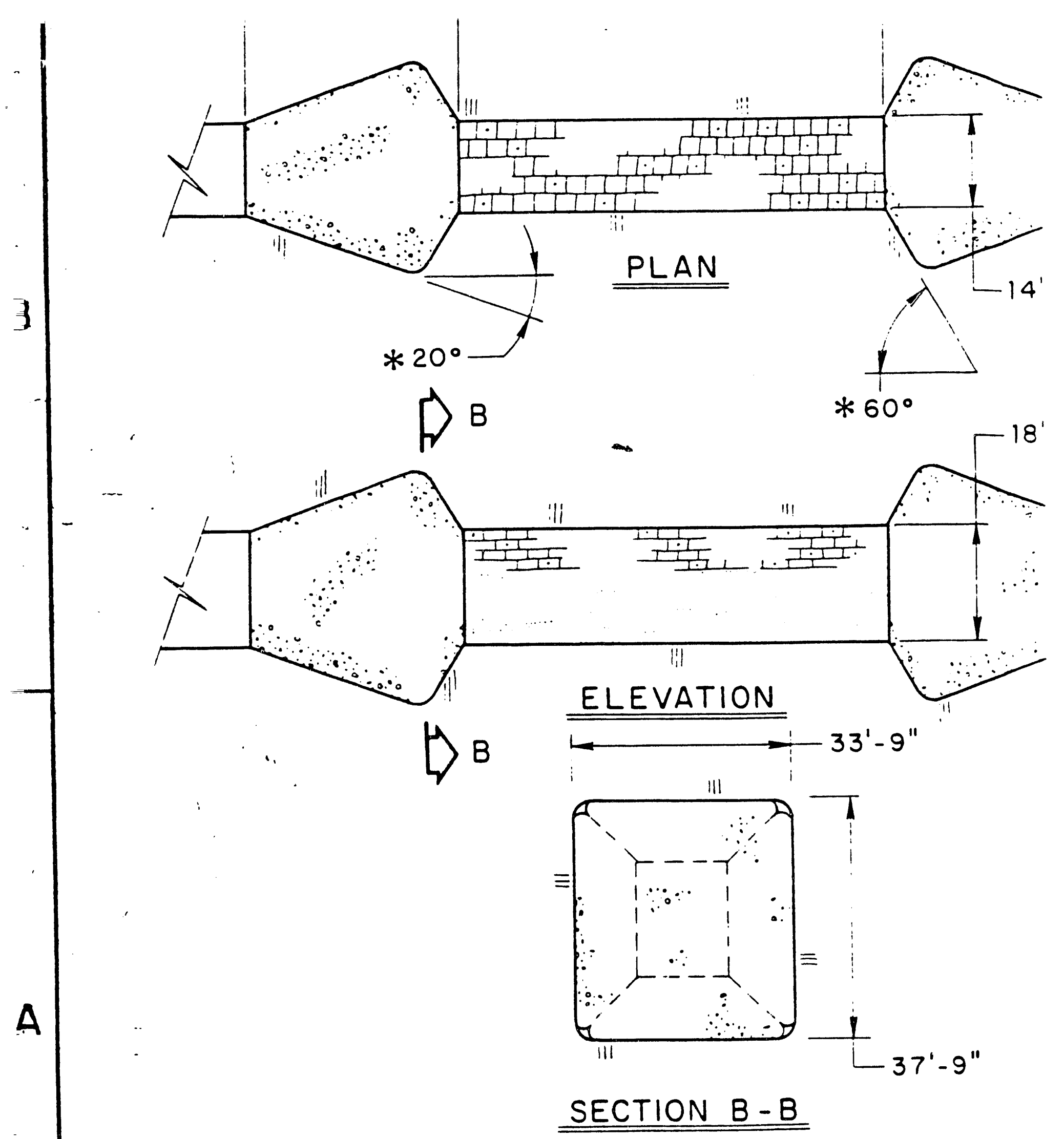




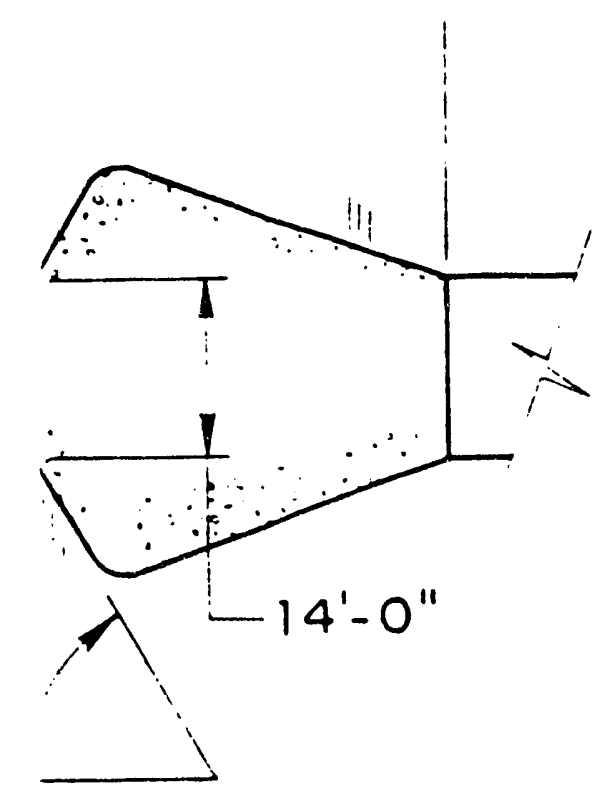

\section{CRUSHED WIPP SALT IDRIFT BACKFILL), TYPICAL}

(FOR DESCRIPIIION OF MATERIAL SEE

NOTE (A5) ON DRAWING NUMBER TRI-46 5)

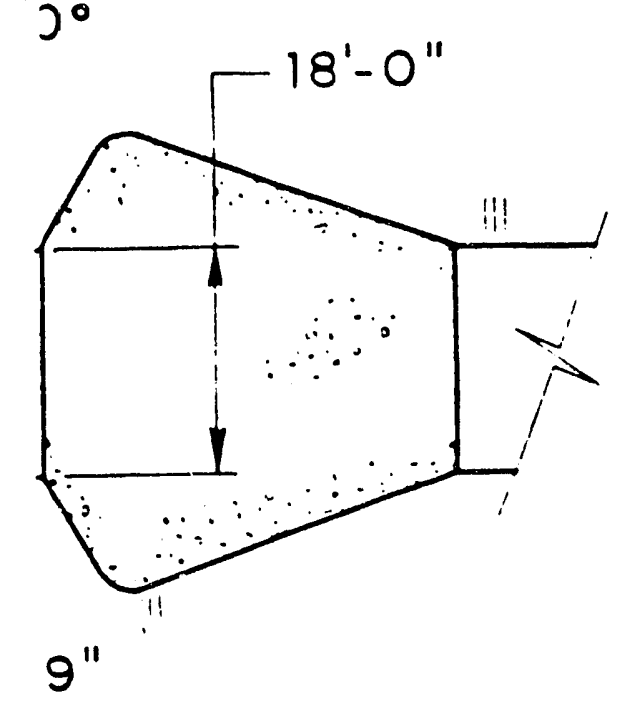

* note:

THESE DIMENSIONS AND ANGLES ARE TYF FOR ALL DRIFT CONFIGURATIONS.

$37^{\prime}-9^{\prime \prime}$

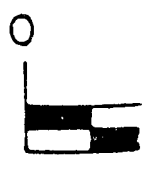




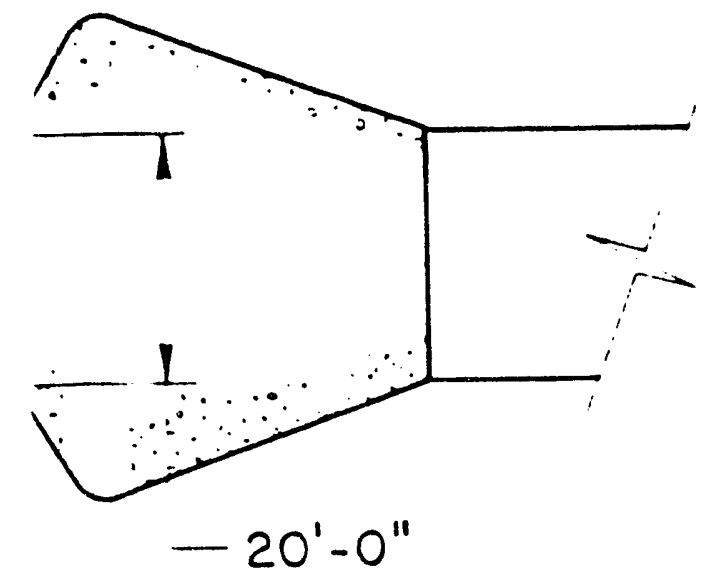

TAMPED SALT 글

\section{DETAIL "B"}

PRECONSOLIDATED SALT, TYPICAL

ALL DRIFTS AND PANEL SEALS

SEE NOTE 1: $3^{\prime \prime}-12^{\prime}-0^{\prime \prime}$

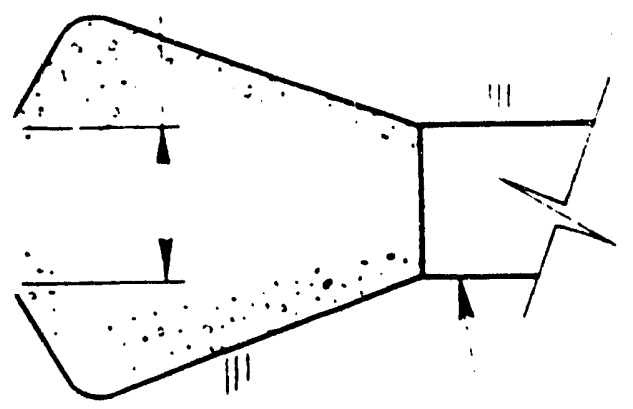

$-9^{\prime \prime}$
CRUSHED WIPP SALT (DRIFT BACKFILL), TYPICAL

IFOR DESCRIPTION OF MATERIAL SEE NOTE AS ON DRAWING NUMIBER TRI $\div 6.51$
- EXCAVATION TOLERAN

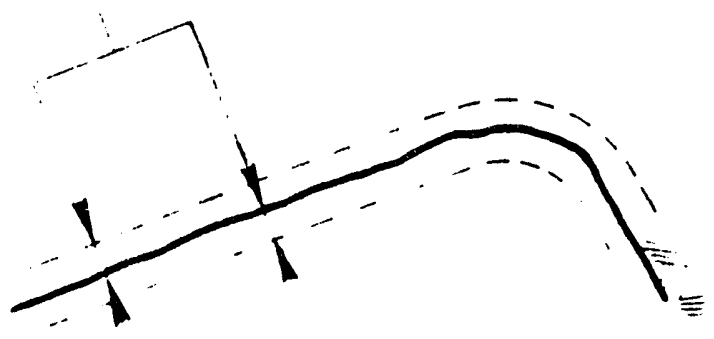

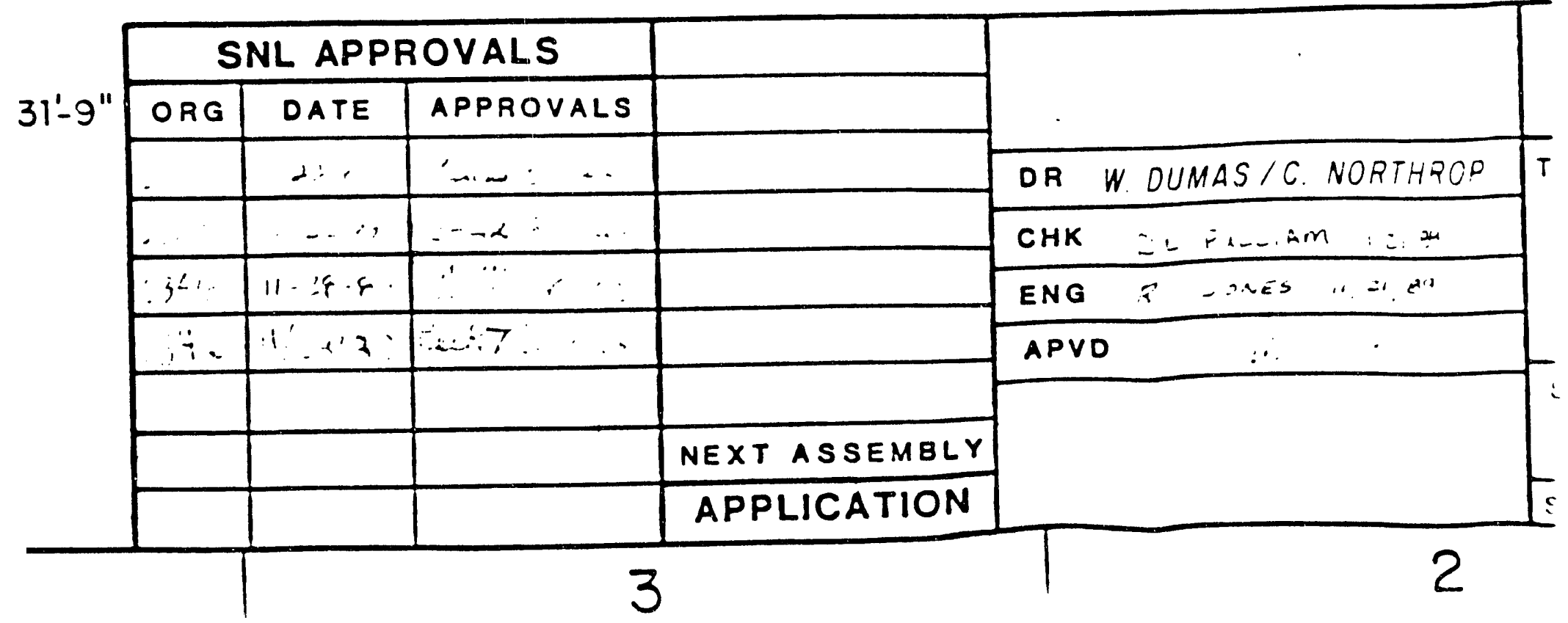


OCKS
SALT

\section{DETAIL "B"}

SOLIDATED SALT, TYPICAL

RIFTS AND PANEL SEALS

SEE NOTE 1;

- EXCAVATION TOLERANCE $\pm 6 "$ TYPICAL

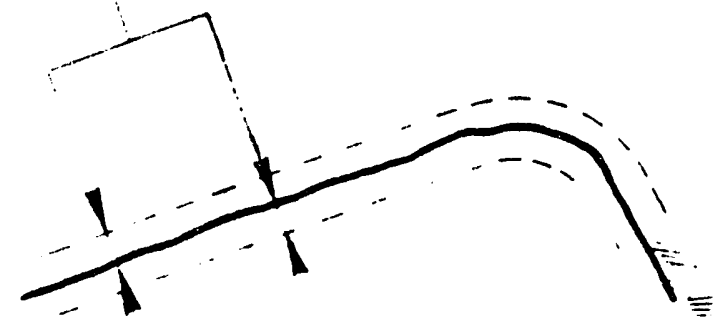

$$
\frac{\text { DETAIL "A" }}{\text { NO SCALE }}
$$

6-5)
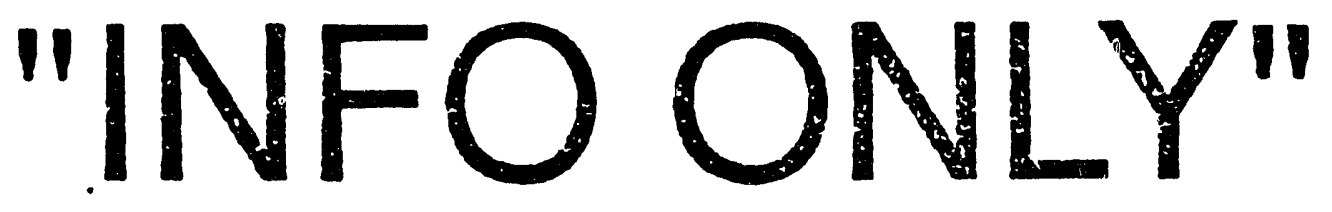

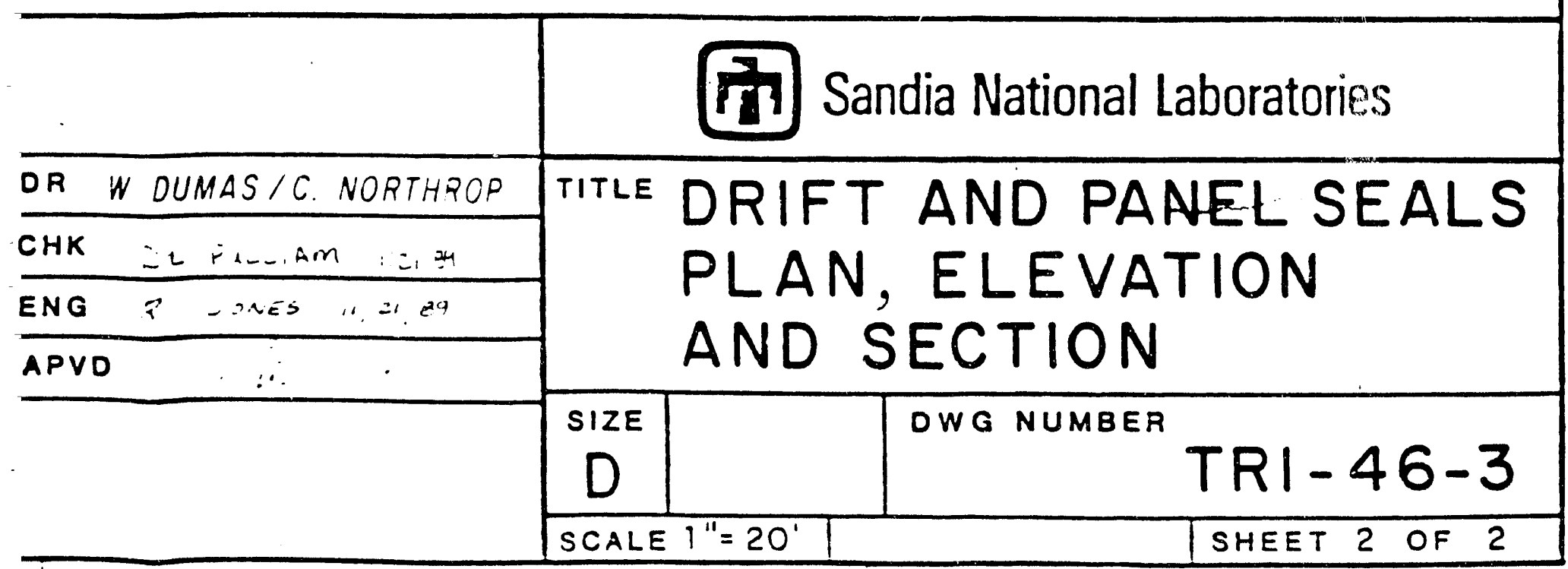




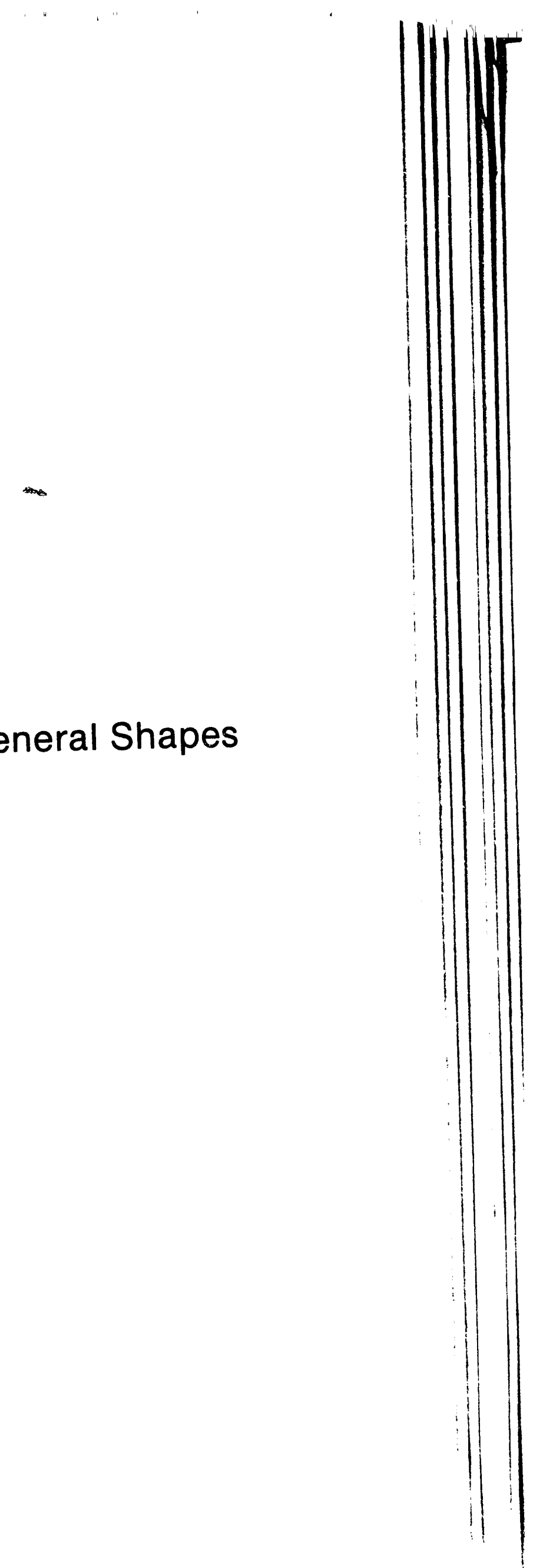




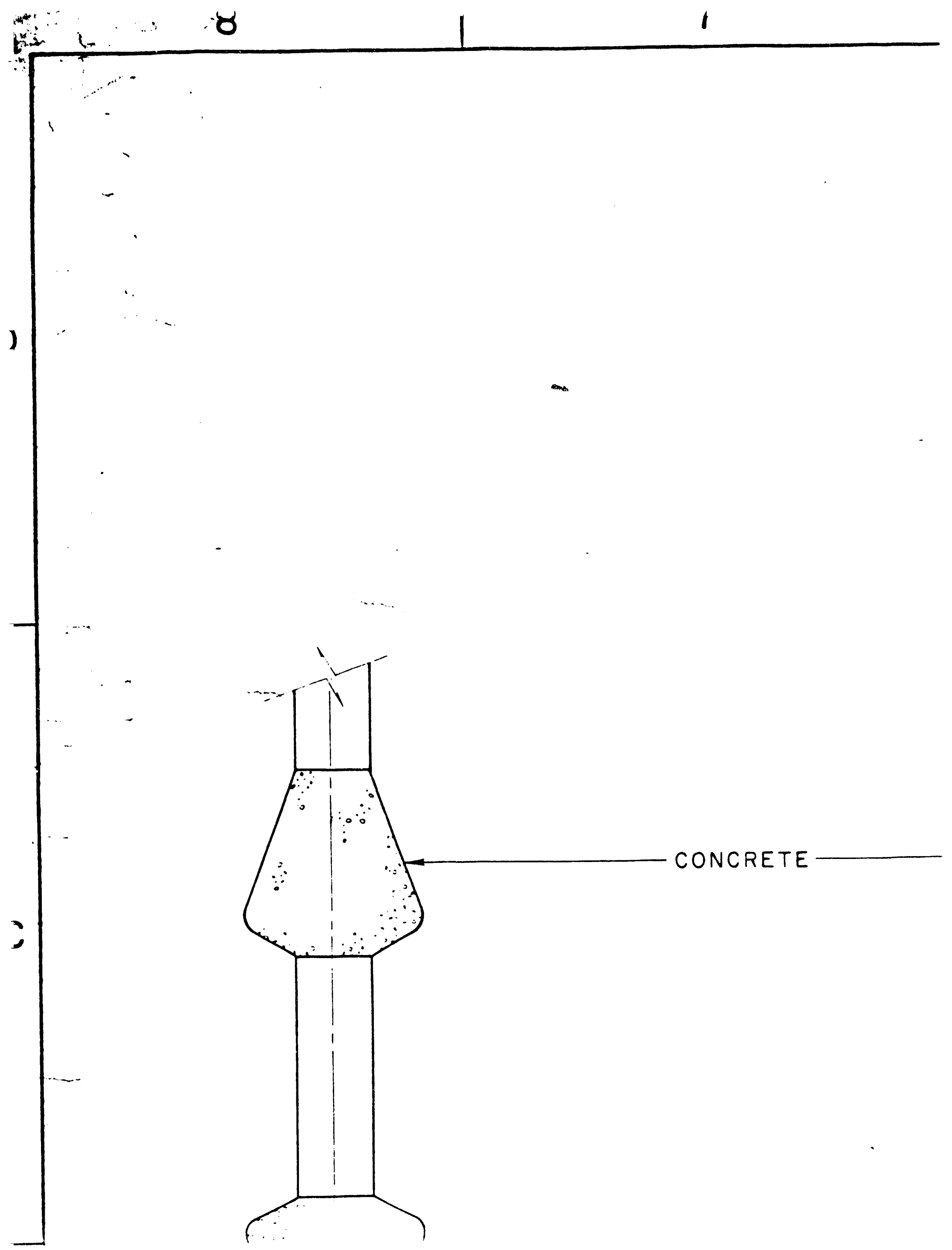



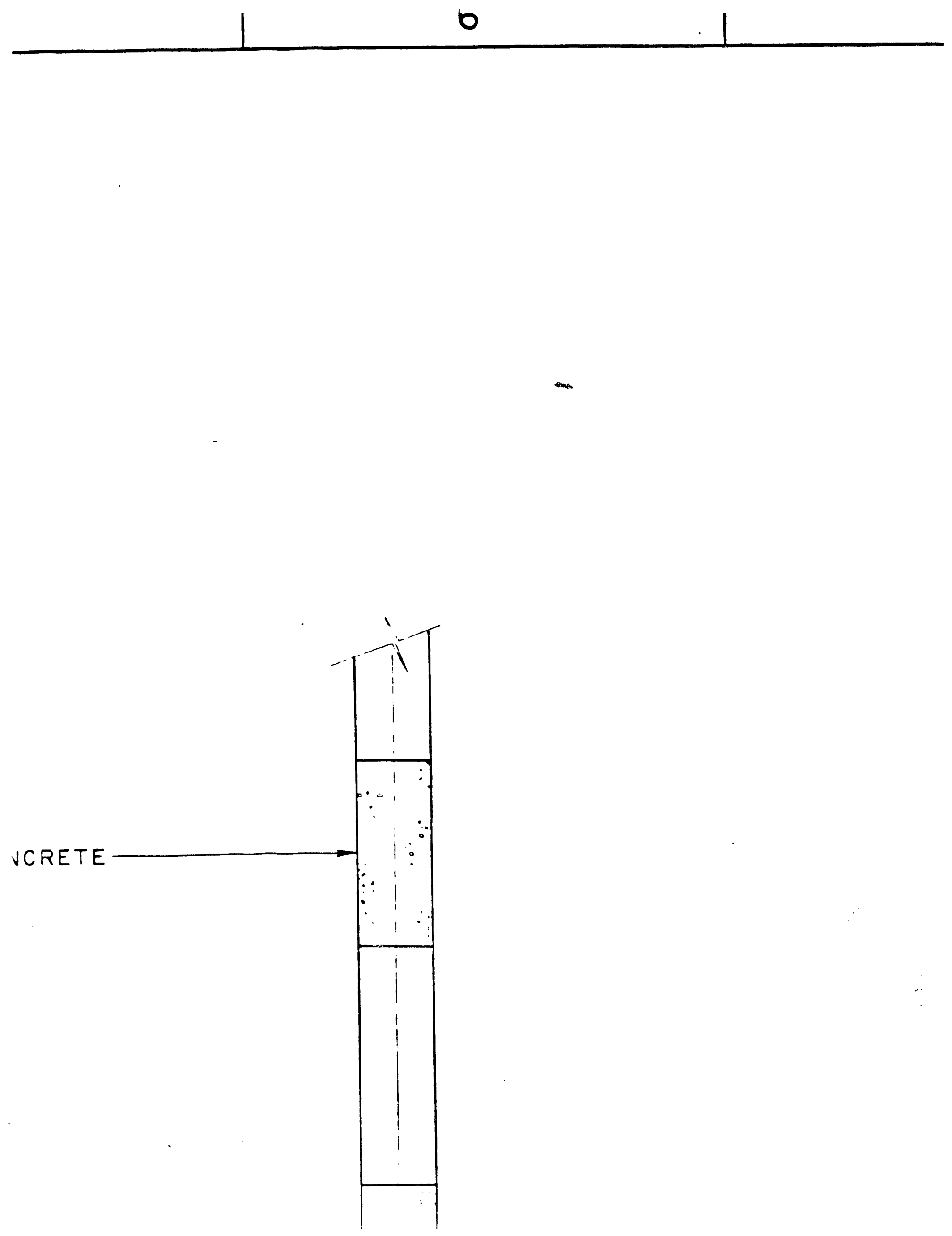


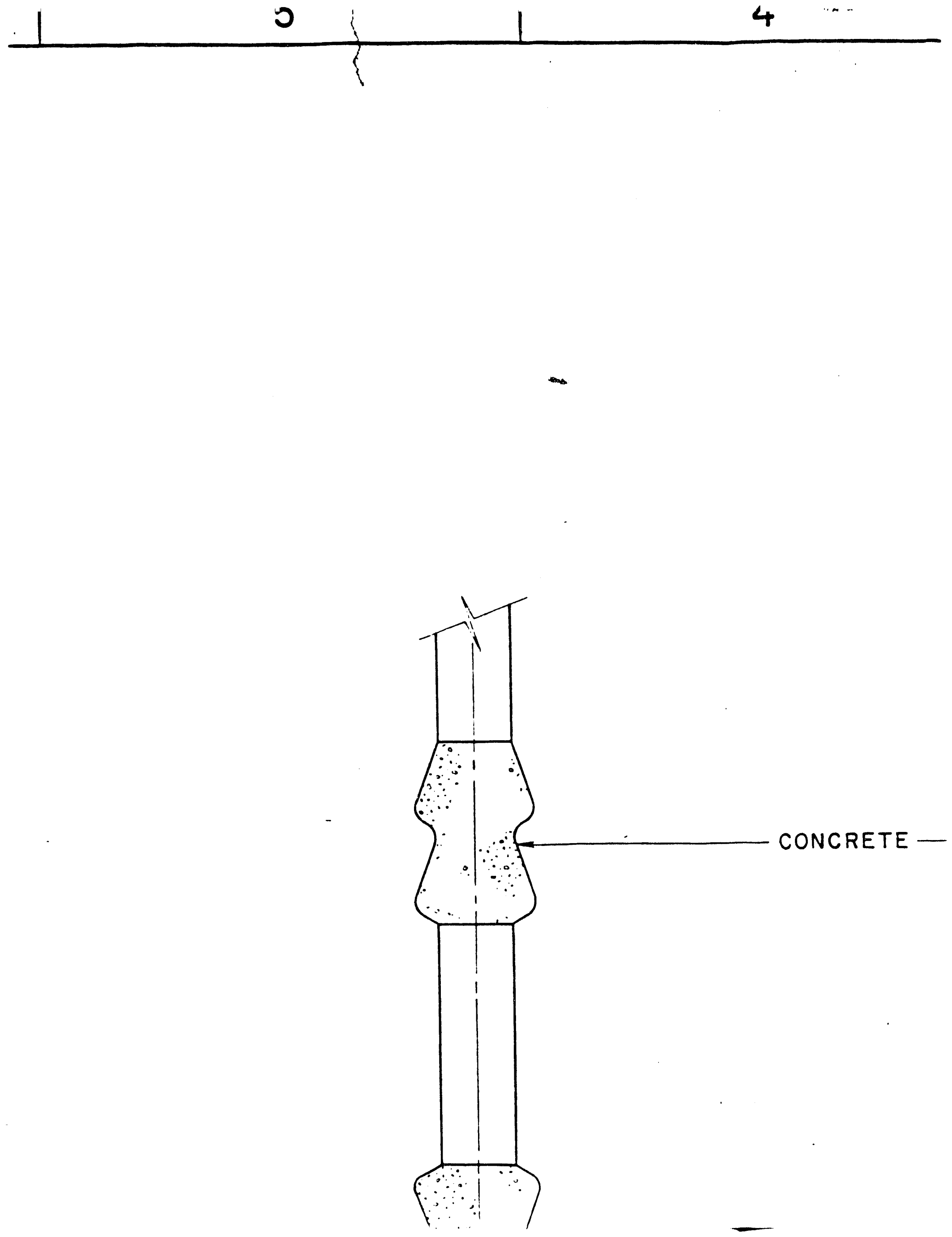



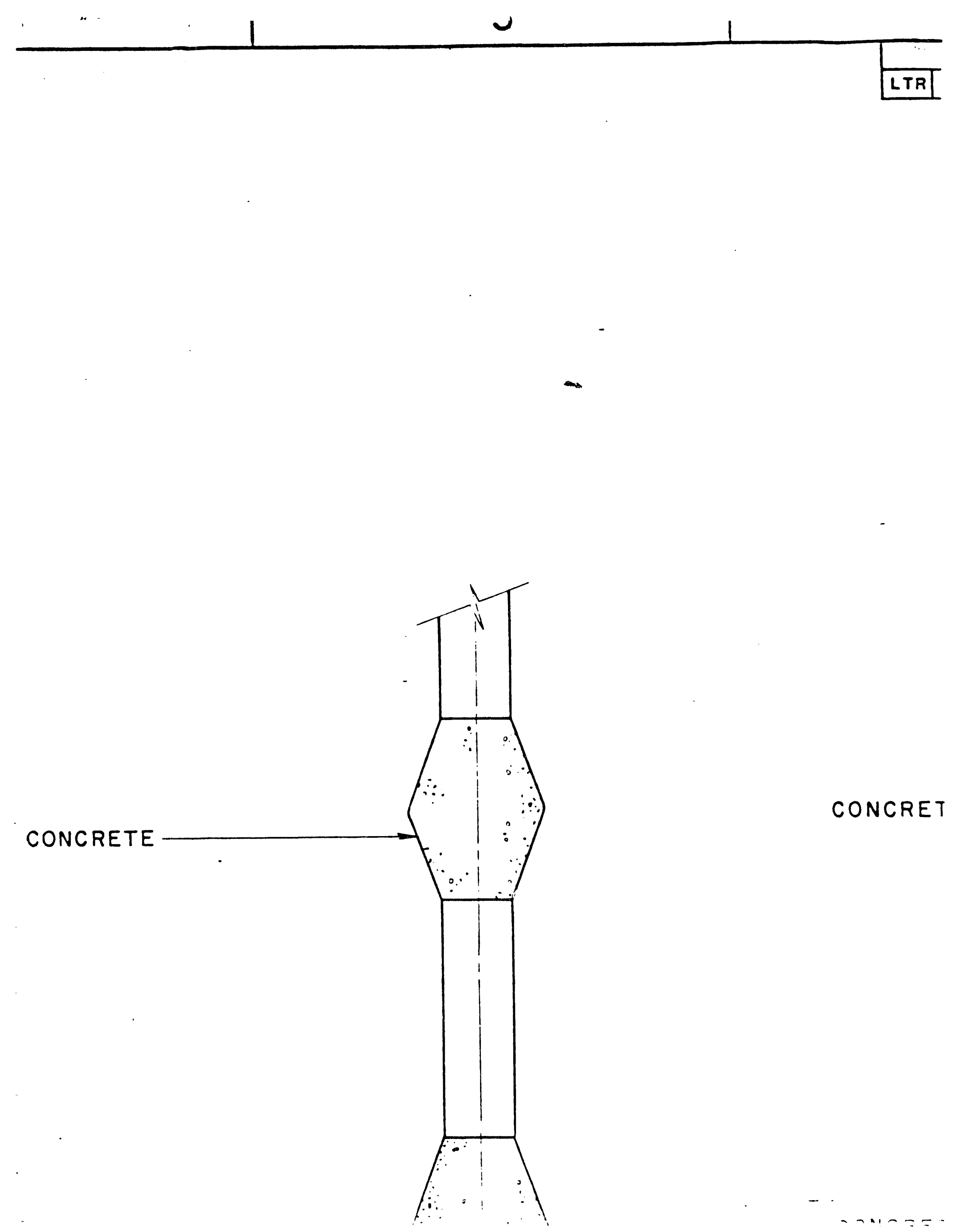



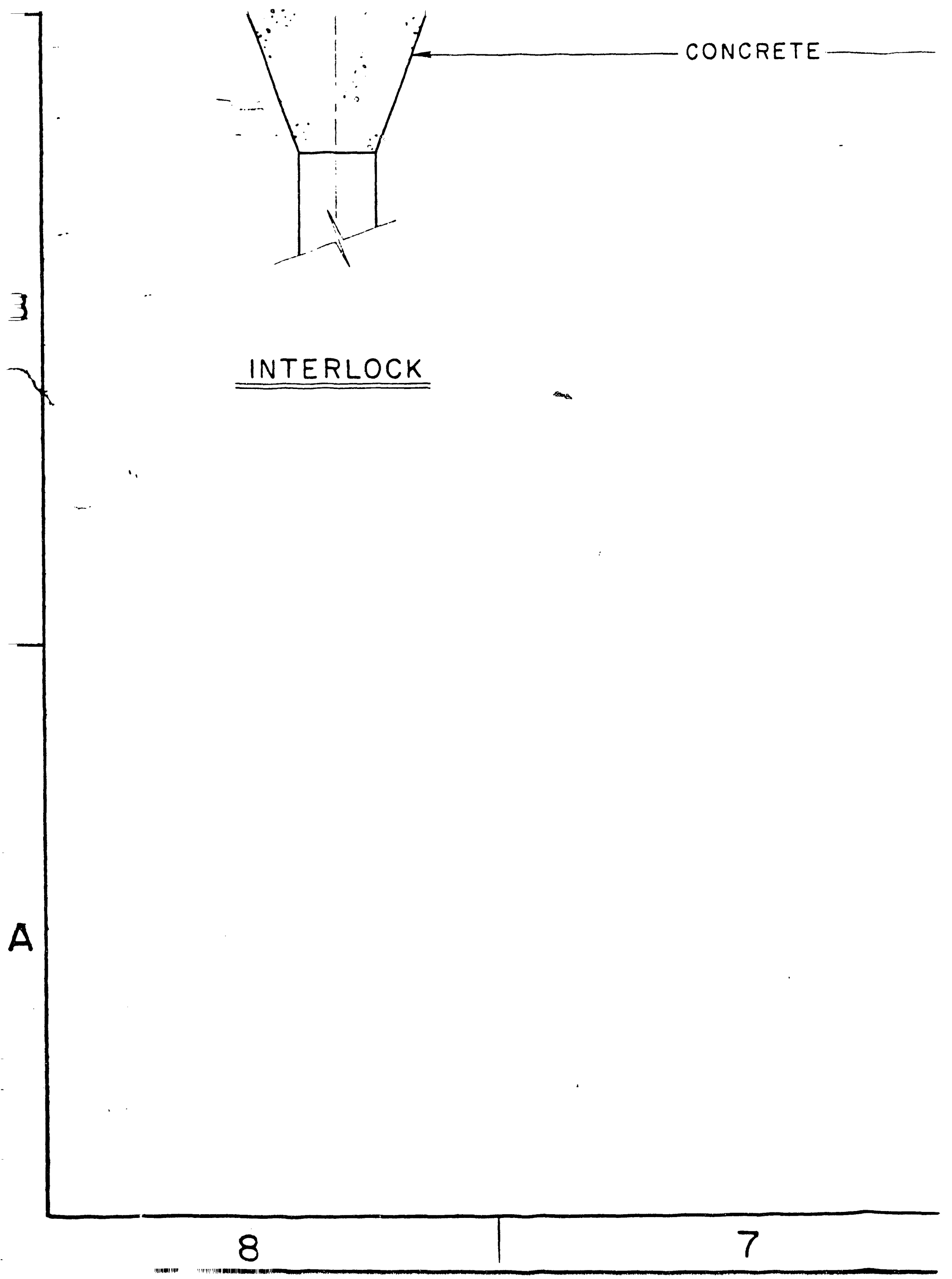


- LUivitiere

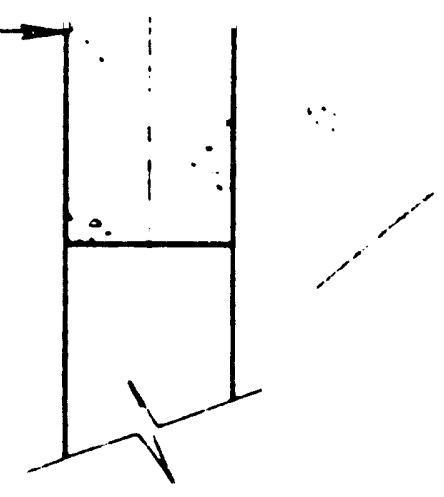

$\because$

PRISM 


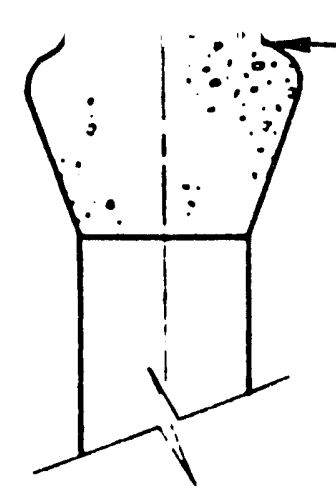

DOUBLE INTERLOCK

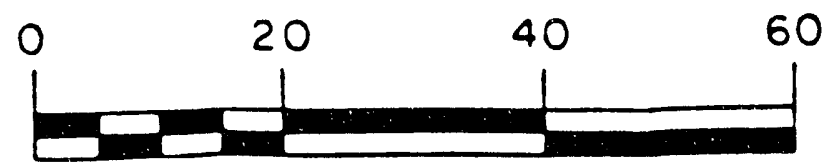

SCALE IN FEET

5 


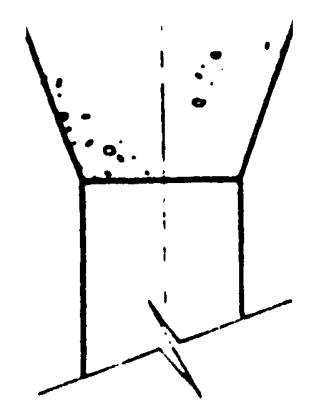

\section{OPPOSED}

\section{TRUNCATED CONES}

NOTES

1. NUMERICAL ANALYSES O' PERFORMANCE WILL BE I SUITED FOR A CONCRETI CURRENT DRAWINGS OF THE "INTERLOCK" SHAPE.

2. ALL SEAL DIMENSIONS AF FURTHER TEST RESUL.TS

\begin{tabular}{|c|c|c|c|c|}
\hline \multicolumn{3}{|c|}{ SNL APPROVALS } & & \\
\hline ORG & DATE & APPROVALS & & \\
\hline$r:$ & $\therefore \lambda \overline{1}$ & 5 C.... & - & DR $W$ \\
\hline 3. & $1112=185$ & isspo' a. an-y & & $\overline{C H K}=$ \\
\hline $634: 5$ & $11-7 \varepsilon-\varepsilon:$ & 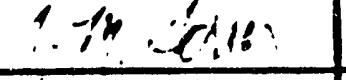 & & ENG \\
\hline $0: 46$ & $11 / 28 / 89$ & Hoe R.Tikin in & & APVD \\
\hline & & & NEXT ASSEMBLY & \\
\hline & & & APPLICATION & \\
\hline
\end{tabular}




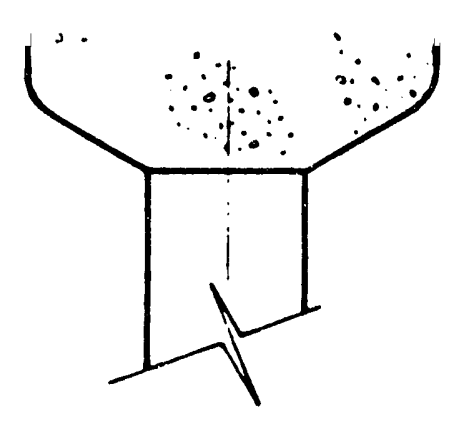

\section{OPPOSED}

INTERLOCK

!ALYSES OF MECHANICAL AND FLOW

WIL'. BE USED TO SELECT THE SHAPE BEST

SONCRETE SEAL DURING CONCEPTUAL DESIGN.

NINGS OF THE SEAL CONCEPTS INCORPORATE :K" SHAPE.

NSIONS ARE SUBJECT TO CHANGE PENDING RESULTS AND ANALYSIS.

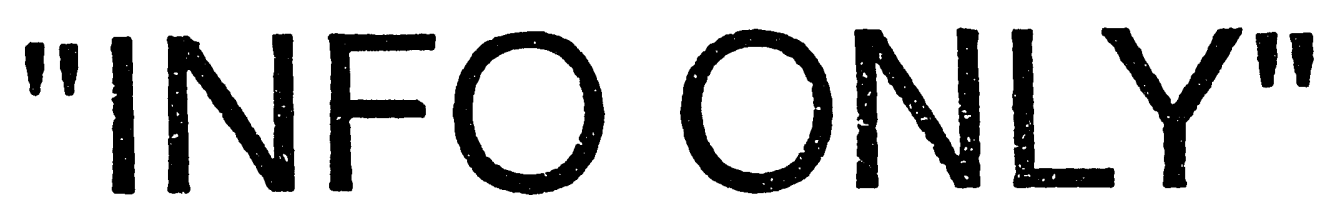

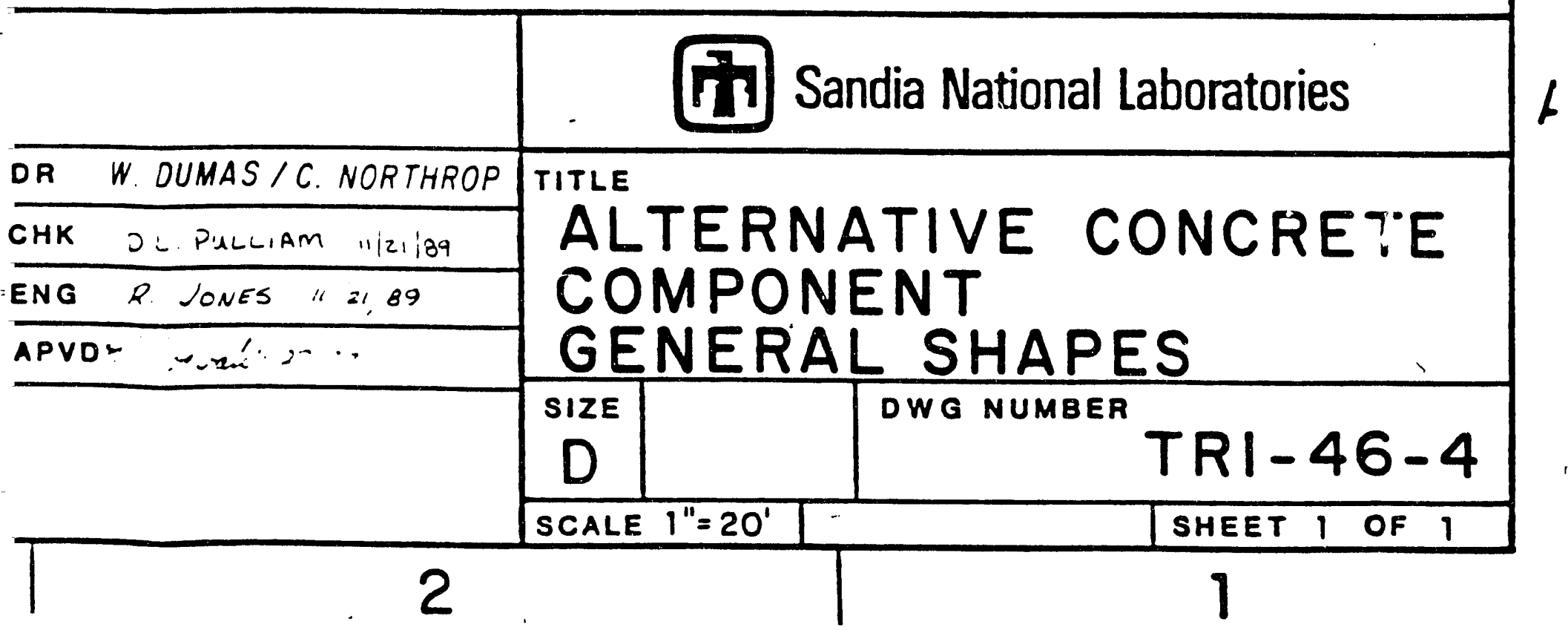




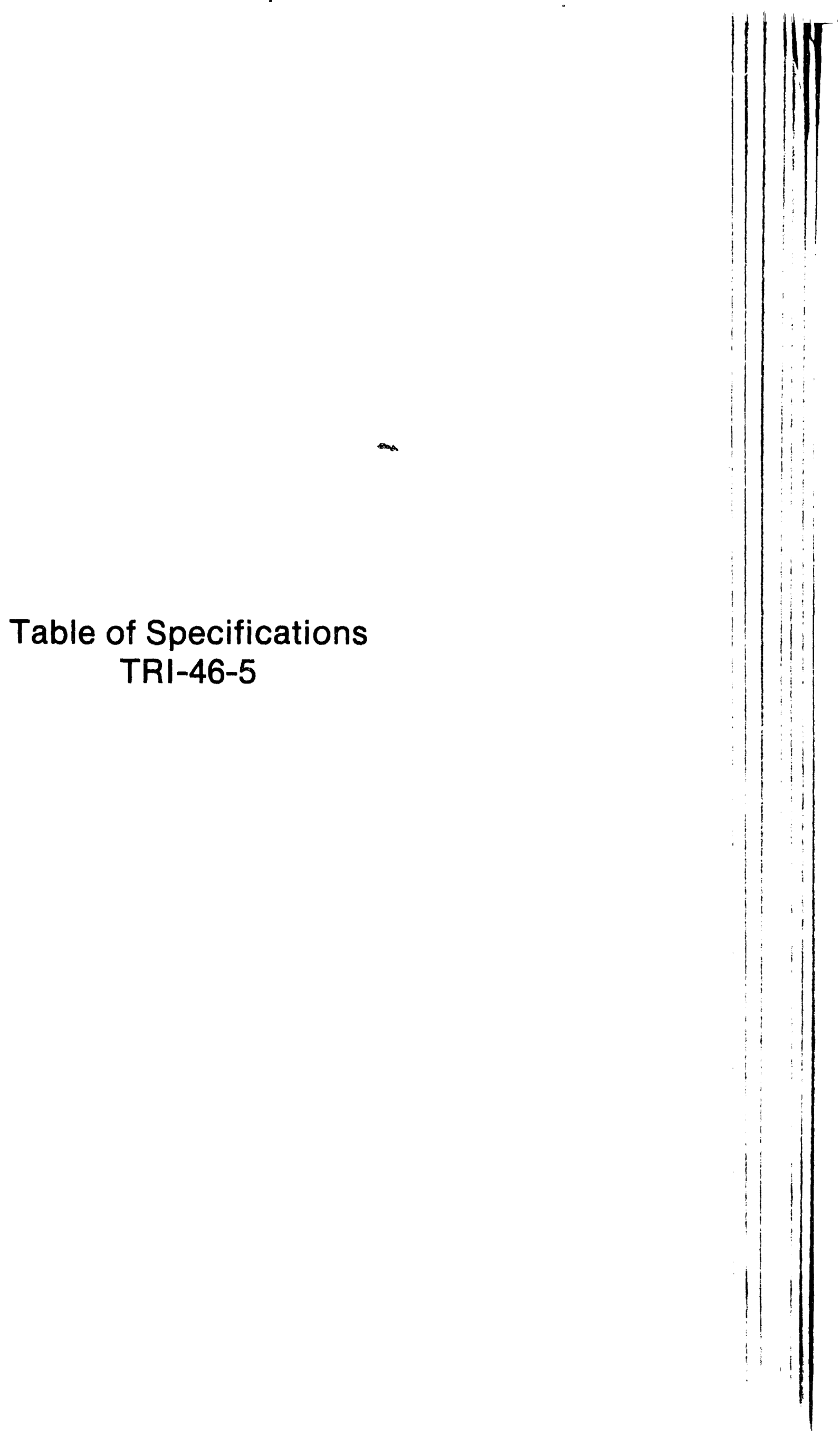




\section{(A) MATERIALS IDENTIFICATION}

CONCRETE

(A) LOWER SHAFT, DRIFT PANEL, AND UPPER SHAFT SEAL COMPONENTS IN THE SALADO FORMATION

THE REFERENCE CONCRETE FORMULATION FOR LOWER SHAFT. DRIFT, AND PANEL SEALS IS AS FOLLOWS (GULICK AND WAKELEY, IN FINAL PREPARATION):

FINE LOCAL (CARLSBAD, NM) AGGREGATE 35 WT\% COARSE LOCAL (CARLSBAD. NM) AGGREGATE 35 WT\% BCT-1F SALT-SATURATED GROUT $30 \mathrm{WT} \%$

THE BCT-1F SALT-SATURATED GROUT FOR THIS REFERENCE CONCRETE FORMULATION WAS DEVELOPED SPECIFICALLY FOR WIPP APPLICATIONS IN THE SALADO FORMATION (GULICK, BOA. AND BUCK, 1980; GULICK AND WAKELEY, IN FINAL PREPARATION): THE BCT-1F GROUT IS PREPARED ACCORDING TO THE FOLLOWING FORMULATION:

CLASS H CEMENT

CLASS C FLY ASH

CAL SEAL (PLASTER)

SODIUM CHLORIDE

DISPERSANT

DEFOAMER

WATER
$48.3 \mathrm{WT} \%$

$16.2 W T \%$

5.7 WT\%

7.9 WT\%

0.78 WT\%

0.02 WT\%

$21.1 \mathrm{WT} \%$

SPECIFICATIONS OF BULK MATERIAL PROPERTIES FOR THIS REFERENCE CONCRETE AFTER CURING (28 DAYS) (GULICK AND WAKELEY, IN FINAL PREPARATION) ARE:

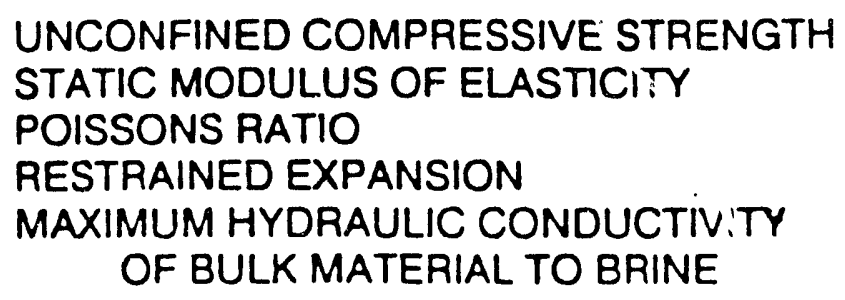

$31 \mathrm{MPa}$

$2.1 \times 10^{4} \mathrm{MPa}$

0.20

0.09

$2 \times 10^{-10} \mathrm{~cm} / \mathrm{s}$

A2) UPPER SHAFT SEAL COMPONENTS IN THE RUSTLER FORMATION AND WATER-BEARING-ZONE SEAL COMPONENTS

THE REFERENCE CONCRETE FORMULATION FOR UPPER SHAFT AND WATER-BEARING ZONE SEALS IS AS FOLLOWS (GULICK AND WAKELEY, IN FINAL PREPARATION):

FINE LOCAL (CARLSBAD, NM) AGGREGATE 35 WT\% COARSE LOCAL (CARLSBAD, NM) AGGREGATE $35 \mathrm{WT} \%$ BCT-1FF GROUT 


\section{CRUSHED SALT}

A- SEAL COMPONENTS IN ALL LOCATIONS

MINED WIPP SALT (FROM THE CREATION OF WI

MINED WIPP SALT MATERIAL TO PASS THROUG SCREEN AND CONFORM TO THE PARTICLE SIZ\& GIVEN IN HOLCOMB AND HANNUM. 1982

AND WAKELEY,

FREE WATER CONTENT: 3 WT\%. TO ACCELERA (HOLCOMB AND SHIELDS. 1987)

35 WT\%

: 35 WT\%

$30 \mathrm{WT} \%$

FERENCE IFICALLY FOR ¿UULICK, BOA. 'REPARATION): THE FOLLOWING

$48.3 W T \%$ $16.2 \mathrm{WT} \%$

5.7 WT\%

$7.9 W T \%$

$0.78 \mathrm{WT} \%$ 0.02 WT\% 21.1 WT\%

$=O R$ THIS ¿GULICK AND

$31 \mathrm{MPa}$

$2.1 \times 10^{4} \mathrm{MPa}$

0.20

0.09

$2 \times 10^{10} \mathrm{~cm} / \mathrm{s}$

ZMATION AND

PPER SHAFT (GULICK AND

35 WT\%

: 35 WT\%

30 WT\%

A3 DRIFT AND PANEL BACKFILL

MINED WIPP SALT (FROM THE CREATION OF WII

FREE WATER CONTENT: 0.5 TO $3.5 W T \%$, TO AC CONSOLIDATION (HOLCOMB AND SHIELDS. 198

INITIAL RELATIVE DENSITY: APPROXIMATELY 6C HOST ROCK SALT, OBTAINED BY EMPLACING IN WITHOUT TAMPING OR USING BLOCKS

INITIAL BULK DENSITY: APPROXIMATELY $1.3 \times 1$

MORE DETAILED SPECIFICATIONS OF COMPOSI SIZE DISTRIBUTION, WATER CONTENT. AND OTI TO BE DEVELOPED DURING FY9O AND FY91 FOF: CONCEPTUAL DESIGN

GROUT

(AE) DISTURBED ROCK ZONES (DRZ) AROUND UPPER SHA

MATERIALS AND TECHNIOUES TO BE DEVELOPE DRAWING ON EXISTING INFORMATION AND CNE CHARACTERIZATION OF DRZ (E.G. PIJSCHET A:

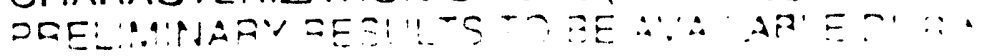




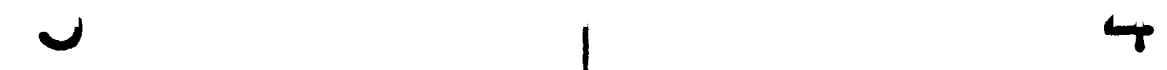

\section{IIONS}

E CREATION OF WIPP EXCAVATIONS)

. TO PASS THROUGH 1/4-INCH MESH THE PARTICLE SIZE DISTRIBUTION NNUM, 1982

$\pi T \%$. TO ACCELERATE CONSOLIDATION 387)

PRECONSOLIDATED TO $80 \%$ OF ILT DENSITY (NOWAK AND STORMONT

$10^{3} \mathrm{~kg} / \mathrm{m}^{3}$

IIONS OF COMPOSITION. PARTICLE CONTENT. AND OTHER PARAMETERS FY9O AND FY91 FOR USE IN THE

JUCTIVITY OF BULK MATERIAL TO

E CREATION OF WIPP EXCAVATIONS)

TO $3.5 \mathrm{WT} \%$, TO ACCELERATE 3 AND SHIELDS. 1987)

APPROXIMATELY $60 \%$ OF INTACT WIPP ) BY EMPLACING INTO DRIFTS G BLOCKS

JOXIMATELY $1.3 \times 10^{3} \mathrm{~kg} / \mathrm{m}^{3}$

IIONS OF COMPOSITION, PARTICLE CONTENT. AND OTHER PARAMETERS FY9O AND FY91 FOR USE IN THE

\section{ROUND UPPER SHAFT SEALS}

S TO BE DEVELOPED AND SPECIFIED. IRMATION AND ONGOING

(E.G.: PIJSCHET AL., 1988);

\section{(C) EMPLACEMENT PROCEDURES}

EMPLACEMENT PROCEDURES AND DEMONSTRATED IN THE SMALL SC ; B, AND C (STORMONT, 1986: STORN: AND HOWARD, 1987); SOME WILL BE SCALE IN SITU TESTS BEGINNING Ai PROCEDURES AND TECHNIQUES H, PROGRAMS (E.G., KELSALL ET AL., 1

(Ci) CRUSHED SALT

PANEL, DRIFT, AND SHAFT STA EMPLACE AND TAMP IN P FILL ACCESS SPACE AT T BLOCKS TO MEET DEINSIT

\section{SHAFT SEALS}

EMPLACE AND TAMP IN P

DRIFT BACKFILL

EMPLACE TO MEET APPA

\section{(C2) SWELLING CLAY MATERIAL}

\section{SHAFT SEALS}

STACK PREFORMED BLOC SPECIFICATION. DEMONS

PERFORMANCE TEST SEF

(C3) CONCRETE

SHAFT SEALS

EMPLACEMENT TECHNIO SMALL SCALE SEAL PERF 1986) WITH IN SITU EMPL VERTICAL BOREHOLES

PANEL, DRIFT, AND SHAFT STA

EMPLACEMENT TECHNIC SMALL SCALE SEAL PERF

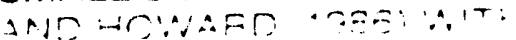




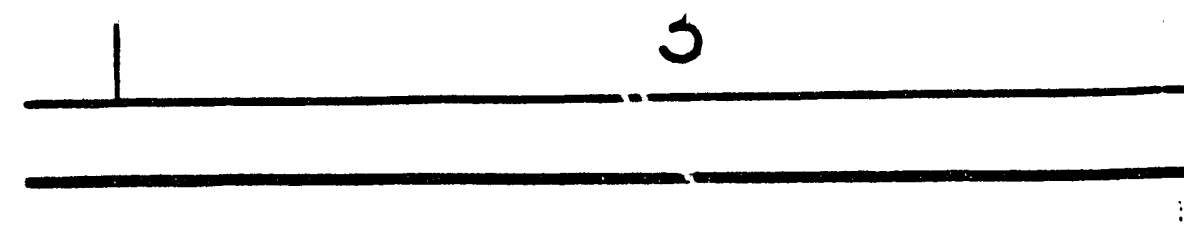

\section{EDURES}

EDURES AND TECHNIQUES HAVE BEEN

tE SMALL SCALE SEAL PERFORMANCE TEST SERIES A. 1986: STORMONT AND HOWARD. 1986. STORI.IONT SOME WILL BE FURTHER DEMONSTRATED ON A LARGE BEGINNING APPROXIMATELY 1/91. EMPLACEMIENT CHNIQUES HAVE BEEN IDENTIFIED IN OTHER SALL ET AL., 196 JA; KELSALL ET AL.. 1985B)

\section{VD SHAFT STATION SEALS}

IND TAMP IN PLACE TO MEET DENSITY SPECIFICATION IS SPACE AT TOP OF DRIFT WITH CRUSHED SALT ) MEET DENSITY SPECIFICATION

IND TAMP IN PLACE TO MEET DENSITY SPECIFICATION

-O MEET APPROXIMATE DENSITY SPECIFICATION

TERIAL

FORMED BLOCKS IN PLACE TO MIEET DENSITY IION. DEMONSTRATED IN SMALL SCALE SEAL NCE TEST SERIES C (STORMIONT AND HOWARD. 1987)

IENT TECHNIQUES HAVE BEEN DEMONSTRATED IN ILE SEAL PERFORMANCE TEST SERIES A (STORMONT. IN SITU EMPLACEMENTS IN 1 METER DIAMETER 3OREHOLES

\section{ND SHAFT STATION SEALS}

\section{(D) PUBLICATIONS}

ARGUELLO. J. G. 1988. Wli OF SEAL COMIPOSITE IT. EVALUATION SAND87-2S ALBUQUEROUE. NM.

BERTRANI-HOWERY. S. G. DISPOSAL-SYSTEM CHAI EVALUATION OF THE WA NATIONAL LABORATORIE

COONS. IV A BERGSTRO J STEADMAN. B. STILLBC OF.THE.ART REPORT ON NUCLEAR WJASTE REPOS FUEL AND WASTE MANAC

GERSTLE W $H$ ANDA $K$ SALT BENTONITE BLOC: ALBUOUERQUE. NM

GULICK. C. W. JR., J. A. BO CEMENT GROUT DEVELS LABORATORIES. ALBUQL

GULICK. C.W. AND L. D $W_{j}$ PROPERTIES OF CEMIEN WASTE ISOLATION PILOT LABORATORIES. ALBUOI

HOLCOMB. D. J. AND D W BACKFILL UNDER CONDI SAND82.0630. SANDIAN,

HOLCOMB. D. J.. AND M. SH OF CAUSHED SALT WITH LABORATORIES. ALBUOL

JACOBSON. A AND R. PUS WASTE PQODUCTS IN EC KBS, STOCKHOLM. SWEL

KELSALL. P C.. D MEYER. DESIGNS FOR PENETRAT BASIN, BMI/ONWII-563. O: $\mathrm{OH}$.

KELSALL. P. C. J. B. CASE. : SCHEMATIC DESIGNS FO PERMIAN BASIN BMI. ON COLUMBUS. OH 


\section{LTR \\ PUBLICATIONS}

DESCRIPTION

DATE

APPROVED

ARGUELLO. J G. IS88. WWIPP PANELENTRYWAY SEAL. NUMERICAL SIR.IULATION OF SEAL CONIPOSITE INITERACTION FOR PRELIR.IINARY SEAL DESIGN EVALUATION SAND87.2804 SANDIA NATIONAL LABORATORIES. ALBUQUEROUE. NM.

BERTRAM-HOWERY. S. G. AND R L. HUNTER. EDS. 1989. PRELIMINARY PLAN FOR OISPOSAL.SYSTEM. 1 CHARACTERIZATION AND LONG.TERM PERFORMANCE EVALUATION OF THE WASTE ISOLATION PILOT PLANT, SAND89-0178. SANDIA NATIONAL LABORATORIES. ALBUQUEROUE. NM.

COONS. IV A BERGSTROM. P GNIRK. M. GRAY. B. KNECHT. R. PUSCH. J. STEADMAN. B. STILLBORG. M. TOKONAMI. AND M. VAAJASAARI. 1987. STATEOF.THE.ART REPORT ON POTENTIALLY USEFUL MATERIALS FOR SEALING NUCLEAR WASTE REPOSITORIES. STRIPA PROJECT 87-12. SWEDISH NUCLEAR FUEL AND WASTE MANAGENIENT CO.. STOCKHOLM. SWEDEN

GERSTLE. W H AND A K JONES. :986. MECHANICAL PROPERTIES QF CRUSHED SALT BENTONITE BLOCKS. SAND86-0707. SANCIA NATIONAL LABORATORIES ALBUQUEROUE. NM.

GULICK. C. W. JR., J. A. BOA. AND A. D. BUCK. :980. BELL CANYON TESTIBCTL CEMAENT GROUT DEVELOPP.IENT REPORT SAND80-1928. SANDIA NATIONAL LABORATORIES. ALBUQUERQUE. NH.

GULICK. C. W. ANDL. D WAKELEY. IN FINAL PREPARATION REFERENCE PROPERTIES OF CER.IENT.BASED PLUGGING AND SEALING MATERIALS FOR THE WASTE ISOLATION PILOT PLANT WIPPI. SAND87-2817. SANDIA NATICNAL LABORATORIES. ALBUOUEROUE. NM

HOLCOMB. D. J. AND D W. HANNUM. 1982. CONSOLIDATION OF CRUSHED SALT BACKFILL UNDER CONDITIONS APPAOPAIATE TO THE WIIPP FACILITY. SAND82-0630. SANDIA NATIONAL LABORATORIES. ALBUQUERQUE. NM

HOLCOMB. D. J.. AND M. SHIELDS. 1987. HYDROSTATIC CREEP CONSOLIDATION OF CRUSHED SALT WITH ADDED WATER, SAND87-1990 SANDIA NATIONAL

- laboratories. albuOUEROUE. NM

JACOBSON A. AND R. PUSCH 1977 DEPOSITION OF HIGH.LEVEL RADIOACTIVE WASTE PRODUCTS IN BOREHOLES WIITH BUFFER SUBSTANCE. KBS REPORT 03. KBS. STOCKHOLM. SWEDEN

KELSALL. P C.. D MEYER. J. B CASE. AND W. E. COONS. 1985A. SCHEMATIC DESIGNS FOR PENETRATION SEALS FOR A REPOSITORY IN THE PARADOX BASIN, BMI / ONWI-563. OFFICE OF NUCLEAR WASTE ISOLATION. COLUMBUS. $\mathrm{OH}$.

KELSALL. P. C. J. B. CASE. W. E. COONS. J. G. FRANZONE. AND D. MEYER. 19858. SCHEMATIC DESIGNS FOR PENETRATION SEALS FOR A REPOSITORY IN THE PERMIAN BASIN, BMI/ONWI-564 OFFICE OF NUCLEAR WASTE ISOLATION. COLUMBUS. OH.

:OWAK E J. AND J. C STORMONT. 1987 SCOPING MODEL CALCULATIONS OF THE AECONISOUIDATICN OF CRUSHED SALT IN THE WIPP SHAFTS, SANDE: C3TS SANDIA HATIC MHAL LABORATCRIES. ALBUOUEROUE. NM.1. 


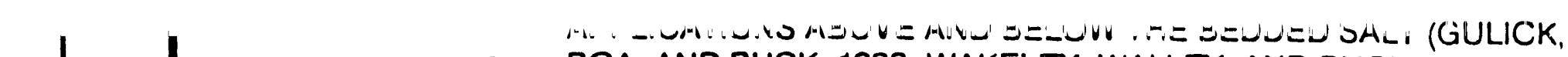
BOA, AND BUCK, 1980; WAKELEY, WALLEY, AND BUCK, 1986; GULICK AND WAKELEY, IN FINAL PREPARATION); THE BCT-1FF GROUT IS PREPARED ACCORDING TO THE FOLLOWING FORMULATION:

CLASS H CEMENT
CLASS C FLY ASH
CAL SEAL (PLASTER)
DISPERSANT
DEFOAMER
WATER

53.1 WT\% 18. 1 WT\% 6.5 WT\% 0.68 WT\% 0.02 WT\% 21.6 WT\%

SPECIFICATIONS OF BULK MATERIAL PROPERTIES FOR THE REFERENCE CONCRETE AFTER CURING (28 DAYS) (GULICK AND WAKELEY, IN FINAL PREPARATION) ARE TO BE:

\author{
UNCONFINED COMPRESSIVE STRENGTH \\ STATIC MODULUS OF ELASTIEITY \\ POISSONS RATIO \\ RESTRAINED EXPANSION \\ MAXIMUM HYDRAULIC CONDUCTIVITY \\ OF BULK MATERIAL TO BRINE
}

$69 \mathrm{MPa}$ $33 \times 10^{3} \mathrm{MPa}$ 0.17

0.03

$2 \times 10 \cdot 10 \mathrm{~cm} ;$ :

NOTE: THE DEVELOPMENT OF CONCRETE FORMULATIONS WILL CONTINUE IN ORDER TO MEET EVOLVING CHEMICAL STABILITY, COMPATIBILITY, AND OTHER PERFORMANCE CRITERIA; NEXT. GENERATION FORMULATIONS WILL BE CHOSEN DURING CONCEPTUAL DESIGN (S. G. BERTRAM-HOWERY AND R. L. HUNTER, 1989)

\title{
SWELLING CLAY
}

\section{ALLLOCATIONS}

THE REFERENCE SWELLING CLAY MATERIAL IS WYOMING (NaRICH) BENTONITE (CONTAINING SMECTITES) (SEE COONS ET AL.. 1987; AND SAWYER AND DAEMEN, 1987 FOR SUMMARY DESCRIPTIONS)

INITIAL DENSITY TAILORED TO CONTROL THE DESIRED SWELLING PRESSURE AT EACH SEAL COMPONENT LOCATION (PUSCH, $1980 \mathrm{~A}$ PUSCH, 1980B) (SEE COONS ET AL., 1987 FOR SUMMARY OF GENERAL SWELLING PRESSURE PROPERTIES)

MAXIMUM HYDRAULIC CONDUCTIVITY OF BULK MATERIAL TO BRINE: $1 \times 10.10 \mathrm{~cm} / \mathrm{s}$

QUANTITATIVE SPECIFICATIONS FOR EMPLACED CLAY MATERIAL (E.G., DENSITY, COMPOSITION, MECHANICAL PROPERTIES) TO BE PROVIDED FOR CONCEPTUAL DESIGN (SEE JACOBSON AND PUSCH. 1977; PUSCH, 1977; PUSCH, 1980A; PUSCH, 1980B SAWYEF. AND DAEMEN, 1987; AND COONS, ET AL.. 1987 FOR BACKGROUND ON THE PROPERTIES AND USES OF BENTONITE FOR SEALING) 


\section{$53.1 W T \%$ \\ 18. 1 WT\% \\ $6.5 W T \%$ \\ $0.68 \mathrm{WT} \%$ \\ 0.02 WT\% \\ 21.6 WT\% \\ =OR THE 'GULICK AND}

SPECIAL MATERIALS AND TECHNIQUES TO BE [ SPECIFIED; COMBINATIONS OF MATERIALS SUC CRUSHED WIPP SALT, BITUMEN. AND CEMENTIT TO BE CONSIDERED ALONG WITH EXISTING INF. PUSCHET AL.. 1988); PRELIMINARY RESULTS TC $12 / 91$

$69 \mathrm{MPa}$

$33 \times 10^{3} \mathrm{MPa}$

0.17

0.03

$2 \times 10^{-10} \mathrm{~cm} / \mathrm{s}$

NS WILL ABILITY, NEXT. ' CONCEPTUAL 39)

OMING ( $\mathrm{Na}$ COONS ET AL.. ARY

RED SWELLING I PUSCH. 1980A; MARY OF

ITERIAL TO

-LAY MATERIAL JERTIES) TO BE SON AND . 1980B SAWYER BACKGROUND IR SEALING)

\section{(B) EQUIPMENT REQUIRED}

\section{(B.) SPECIALTY EQUIPMENT}

BLOCK MANUFACTURE EQUIPMENT FOR CRUSHED $S$ SWELLING CLAY MATERIAL: EXISTING EQUIPMENT M, COMPACT SYSTEMS, ALBUQUERQUE, NM (GERSTLE STORMONT AND HOWARD, 1987) REPRESENTS A PRE EQUIPMENT SPECIFICATION: ADOITIONAL EVALUATIC MANUFACTURE EQUIPMENT TO BE CARRIED OUT TC ADEQUATE PRODUCTION RATE CAPABILITY AND COI FINAL SPECIFICATIONS FOR BLOCKS

\section{(EZ) CONVENTIONAL EQUIPMENT}

\section{TO BE SPECIFIED DURING CONCEPTUAL DESIGN}

FRONT-END LOADER OR PNEUMATIC STOWING CRUSHED SALT EMIPLACEMENT

COMPRESSED-AIR-DRIVEN MECHANICAL TAMIPI FOR CRUSHED SALT

CONCRETE EMPLACEMENT EQUIPMENT (EQUIF DESCRIBED IN STORMONT. 1986. STORMIONT AI

GROUT EMPLACEMENT EOUIPMENT (SOME EOI ARE DESCRIBED IN PUSCH ET AL., 1988)

DRUM-TYPE MECHANICAL MINER FOR ADDITIOR IN DRIFTS

EXCAVATION EOUIPMENT FOR SHAFT TED 
VER SHAFT, DRIFT, AND PANEL SEALS

ALS AND TECHNIQUES TO BE DEVELOPED AND BINATIONS OF MATERIALS SUCH AS WET SALT. BITUMEN. AND CEMENTITIOUS MATERIALS ZED ALONG WITH EXISTING INFORMATION (E.G. 388); PRELIMINARY RESULTS TO BE AVAILABLE
(C) GROUT

UPPER SHAFT SEF

EMPLACE GF

TECHNIQUES

EXISTING INF

RESULTS TO

PANEL, DRIFT, ANC

SPECIAL TEC COMBINATIO

SALT. BITUMI CONSIDERE[ ET AL.. 1988).

IE EQUIPMENT FOR CRUSHED SALT AND ERIAL: EXISTING EQUIPMENT MANUFACTURED BY ALBUQUERQUE, NM (GERSTLE AND JONES, 1986; IARD, 1987) REPRESENTS A PRELIMINARY ¿ATION: ADDITIONAL EVALUATION OF BLOCK PMENT TO BE CARRIED OUT TO ASSURE ION RATE CAPABILITY AND COMPATIBILITY WITH IS FOR BLOCKS

ミNT

IING CONCEPTUAL DESIGN

IDER OR PNEUMATIC STOWING EOUIPMENT FOR EMPLACEMENT

IIR-DRIVEN MECHANICAL TAMPING EQUIPMENT SALT

'LACEMENT EQUIPMENT (EQUIPMENT TYPES ;TORMONT. 1986. STORMONT AND HOWARD. 1986)

:EMENT EOUIPMENT (SOME EQUIPMENT TYPES ) IN PUSCH ET AL., 1988)

SHANICAL MINER FOR ADDITIONAL EXCAVATIONS JUIPMENT FOR SHAFT TBD 
AND HOWARD, 1986) WITH IN SITU EMPLACEMENTS IN $\overline{1}$ METER

DIAMETER HORIZONTAL BOREHOLES

\section{UPPER SHAFT SEALS}

EMFLACE GROUT UNDER PRESSURE AT SEAL LOCATIONS

TECHNIQUES TO BE DEVELOPED AND SPECIFIED. DRAWING ON

EXISTING INFORMATION (E.G., PUSCH ET AL.. 1988): PRELIMINARY

PIJSC

CH:

BE:

RESULTS TO BE AVAILABLE DUFING CONCEPTUAL DESIGN

\section{PANEL, DRIFT, AND SHAFT STATION SEALS}

SPECIAL TECHNIQUES TO BE DEVELOPED AND SPECIFIED:

COMBINATIONS OF MATERIALS SUCH AS WET CRUSHED W'IPP

SALT. BITUMEN. AND CEMENTITIOUS MATERIALS TO BE

CONSIDERED ALONG WITH EXISTING INFORMATION (E.G.. PUSCH

ET AL.. 1988); PRELIMINARY RESULTS TO BE AVAILABLE 12.91

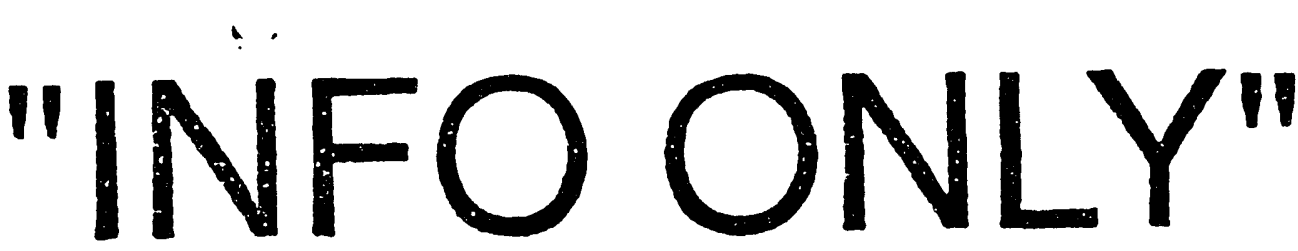

\begin{tabular}{|c|c|c|c|c|}
\hline \multicolumn{3}{|c|}{ SNL APPROVALS } & & \\
\hline ORG & DATE & APPROVALS & & \\
\hline & $\cdots$ & $\cdots \cdots \cdot$ & & DR C NORTHACP \\
\hline & & $\cdots$ & & CHK $\therefore-P U$ CAM $\because=$ \\
\hline$\cdots$ & $; \quad \because$ & $\because \because$ & & $E N G \because$ - ONES $" \therefore$. 39 \\
\hline$\cdot$ & $\therefore$ & $\therefore \quad-\quad-$ & $\therefore$ & APVD \\
\hline & & & NEXT ASSEMBLY & \\
\hline & & & APPLICATION & \\
\hline
\end{tabular}


DOWERS. D W. S. J. LAMBERT S.E. SHAFFER, L. R. HILL. W. D. WEART. EDS. 1978 GEOLOGICAL CHARACTERIZATION AEPORT, WASTE ISOLATION PILOT PLANT WIPP) SITE. SQUTHEASTEAN NEW MEXICO, VOL. 1. SAND 78.1596. SANDIA NATIONAL LABORATORIES. ALBUOUEROUE. NM.

PUSCH. R. $197 \%$ REQUIRED PHYSICAL AND MECHANICAL PROPERTIES OF BUFFER MASSES. KBS REPORT 03. KBS. STOCKHOLM SWEDEN

FIJSCH R GBCA WATER IJPTAKE MIIGRATION AND SWELLING CHARACTEPISTICS OF UPISATURATED ANND SATURATED HIGHLY COR.IPACTED BENTONITE. KBS REPORT 8O-11 KBS. STOCKHOLM SVIEDEN

PUSCH. R.. 19808 S..ELLING PRESSURE OF HIGHLY COP.IPACTED EENTONITE, KBS R.EPOAT BC- :3. KBS STOCKHOLM SWJEDEN.

PUSCH. R . L. BORGESSON A FREDERIKSON. I. MARKSTROM. M ERLSTROM. G. RAMOVIST IN GRAY W CCONS. AND M ONOFREI. 1988. ROCK SEALIPIG. NTTERINI REPORT OIJ THE ROCK SEALING PROJECT ST AGE I) STRIPA PROJECT SQ.11 SWEDISH NUCLEAR FUEL AND WASTE MANAGEMENT CO . STOCKHOLM SIVEDEN

SAIVYER. W D.. 11 . AND J J K. DAENIEN :987 EXPERIMIENTAL ASSESSMENT OF THE SEALING PERFORR.IANCE OF BENTONITE BOREHOLE PLUGS.

NLIREG;CR-4995. PREPARED FOR U S NUCLEAR REGULATORY COMMISSION WASHINGTON DC.

STORMONT J C . ED :986 DEVELOPMMENT AND IMIPLEMIENTATION TEST SERIES A OF THE SI:@ALL.SCALE SEAL PEFFORA.1ANCE TESTS, SAND85.2802 SAIJDIA NATIONAL LABORATCRIES. ALBUOUEROUE. NM

STORMIONT J C AND C L. HOWARD. 1986. DEVELOPMENT AND IMPLEMIENTA. TION TESI SERIES B OF THE SMIALL-SCALE SEAL PERFORMANCE TESTS, SANU86-132G SAMPIA NATIONAL LABORATORIES. ALBUQUEROUE. NM

STOR .MONT. J C.. AND C. L. HOWARD. 1387. DEVELOPMENT IMPLEMENTATION AND EARLY RESULTS TEST SERIES C OF THE SMALL SCALE SEAL PERFORMANCE TESTS. SAND87.2203 SANDIA NATIONAL LABOPATORIES. ALBUOUERDUE. NM

U S DEPARTM.9ENT OF ENIERGY $1 \because 6$ WIPP DESIGN VALIDATION FINALL REPORT, DOE.WIPP 86.C'C BECHTEL NATIONAL INNC SAN FRAPICISCO CA

$\therefore A K E L E Y ~ L D$ D $1.1 \because$ WLLEY AND A D BUCK. 1986 DEVELOPAENT OF FRESHWATER GFQLT SUESEQUENT TO THE BELL CANYON TESTS 'BCT) :. ISCELLANEOUS PAPER SL.Ẽ.2 US ARMY ENGINEER WATERWAYS EXPERIAENT STATION VICKSBURG MS

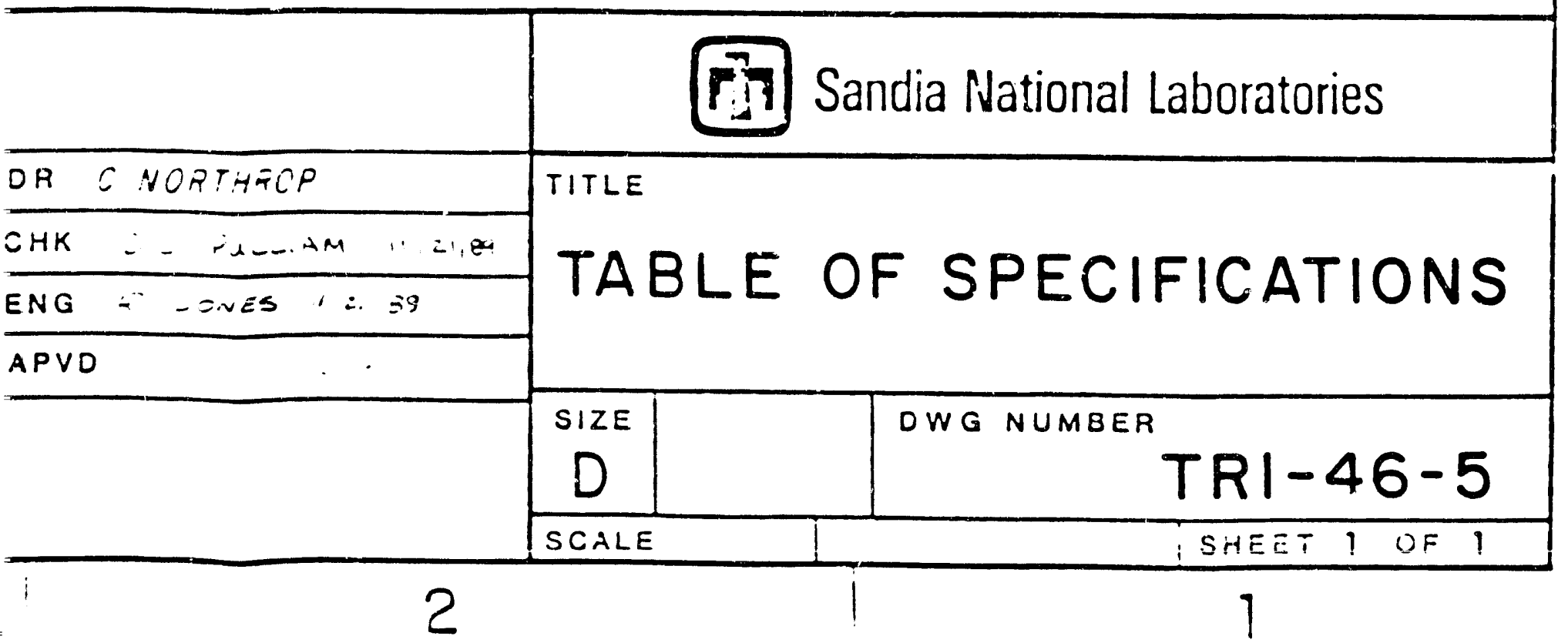


Figure 7.2-8

Well Control Base Map

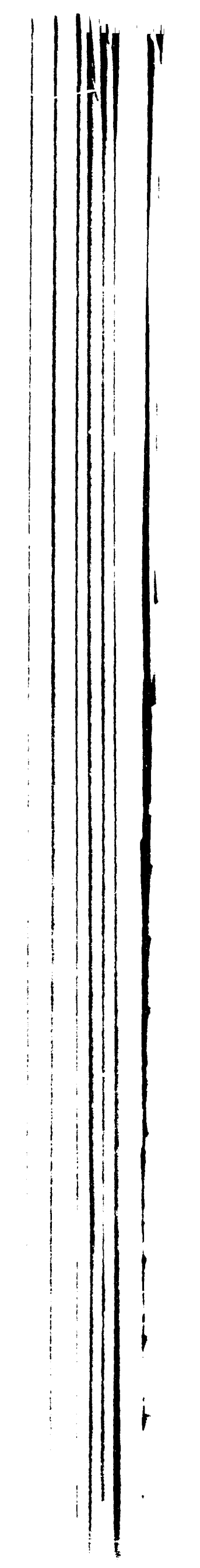


R 30 E

R $31 \mathrm{E}$

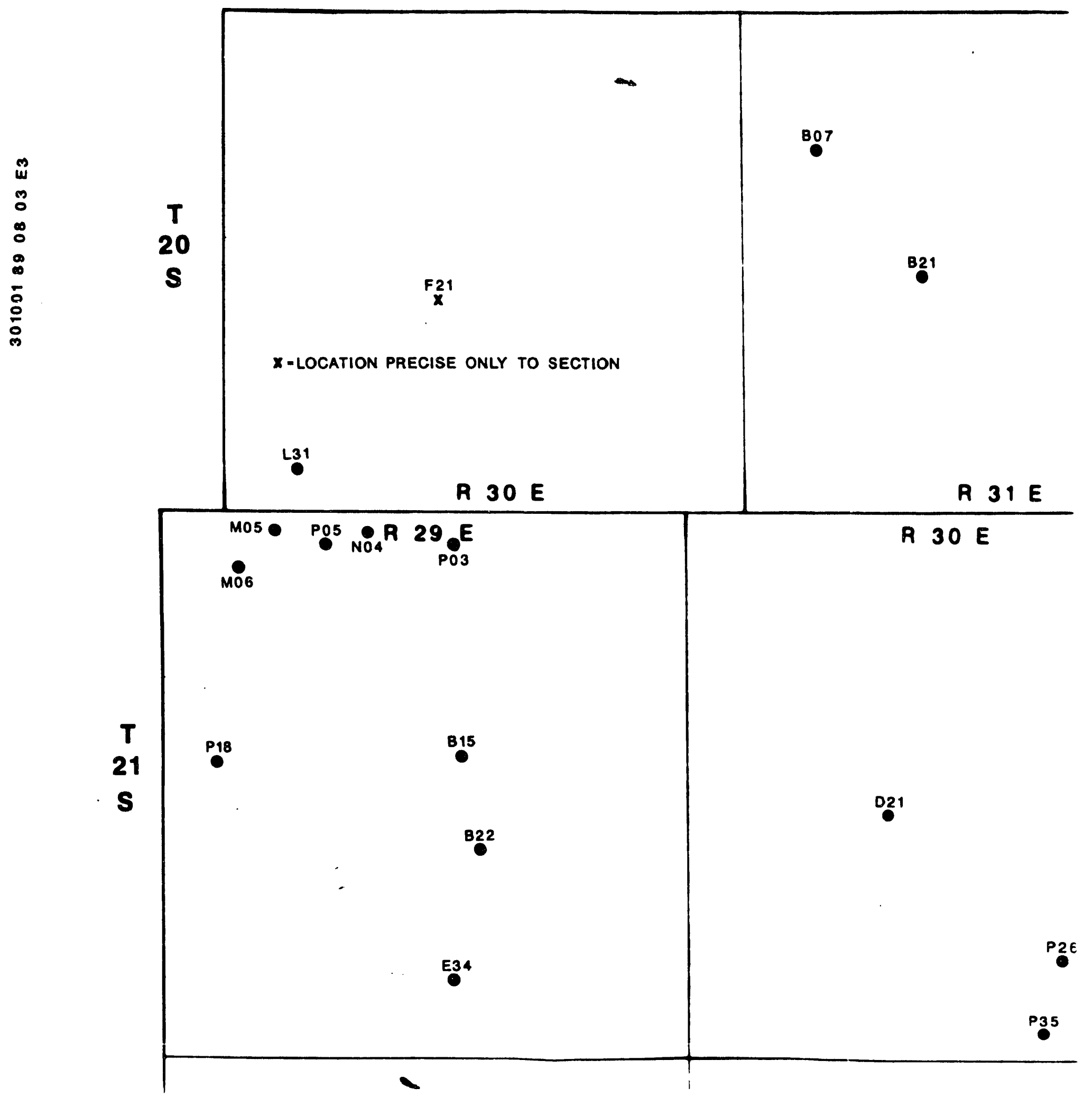




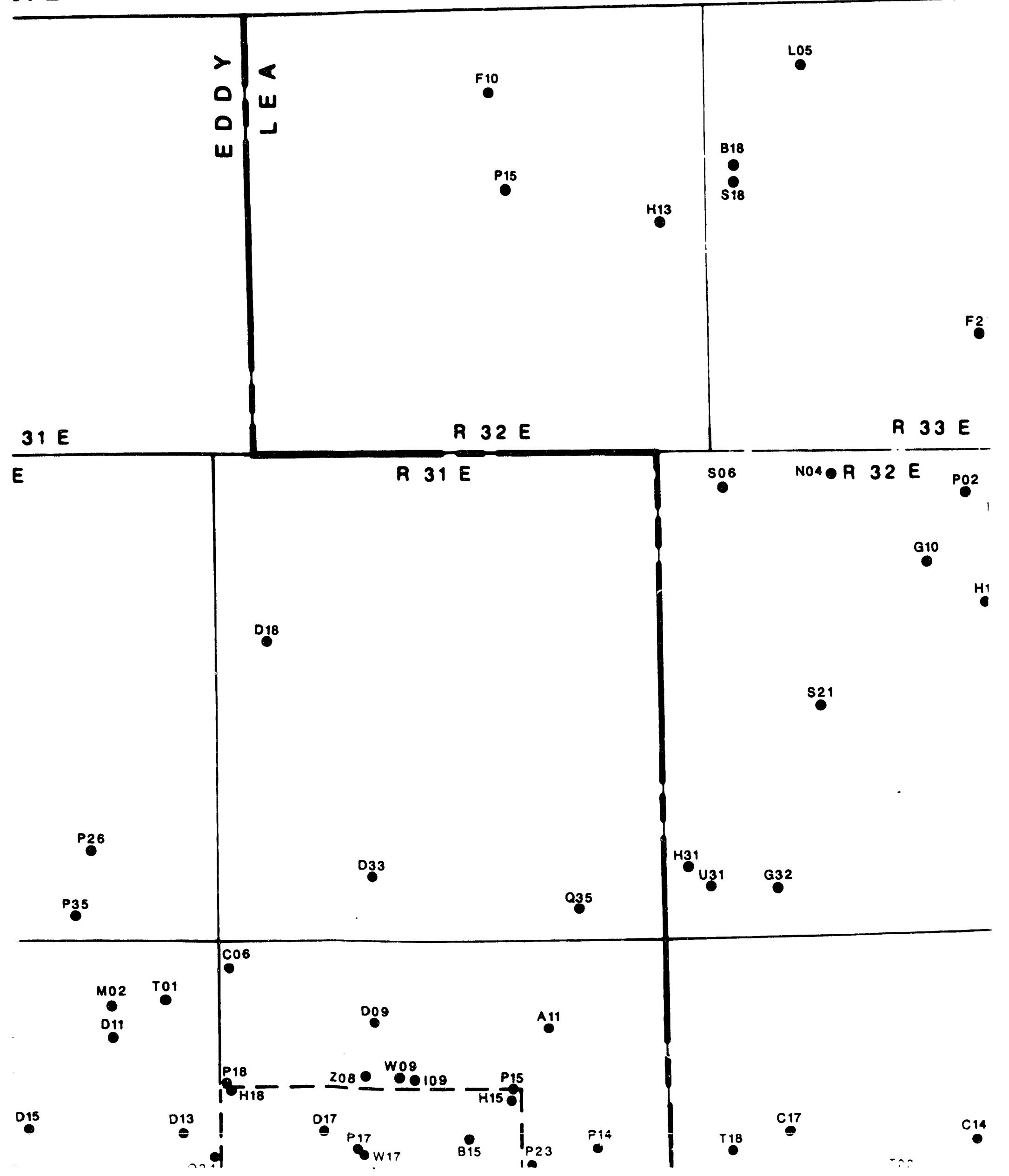




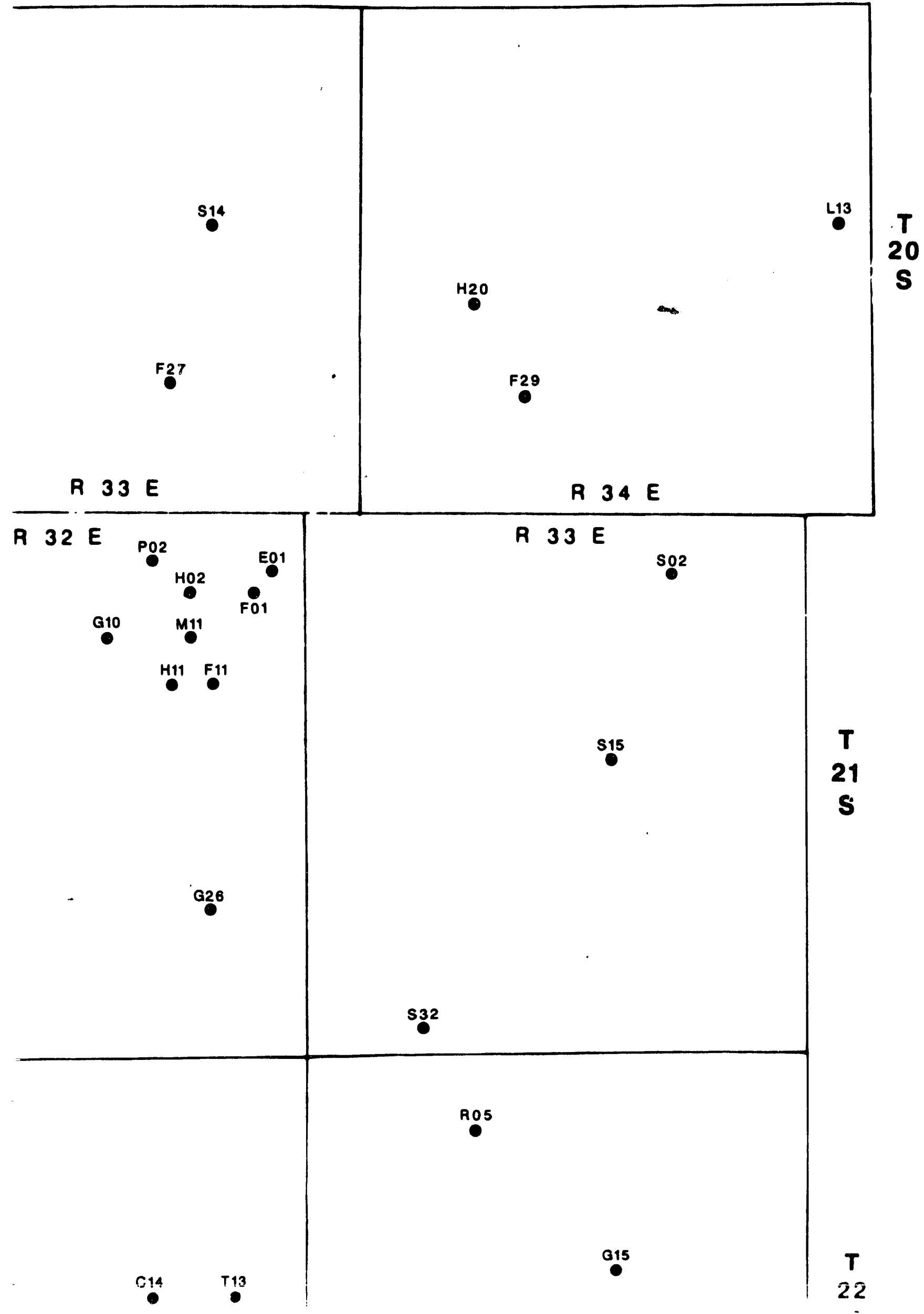




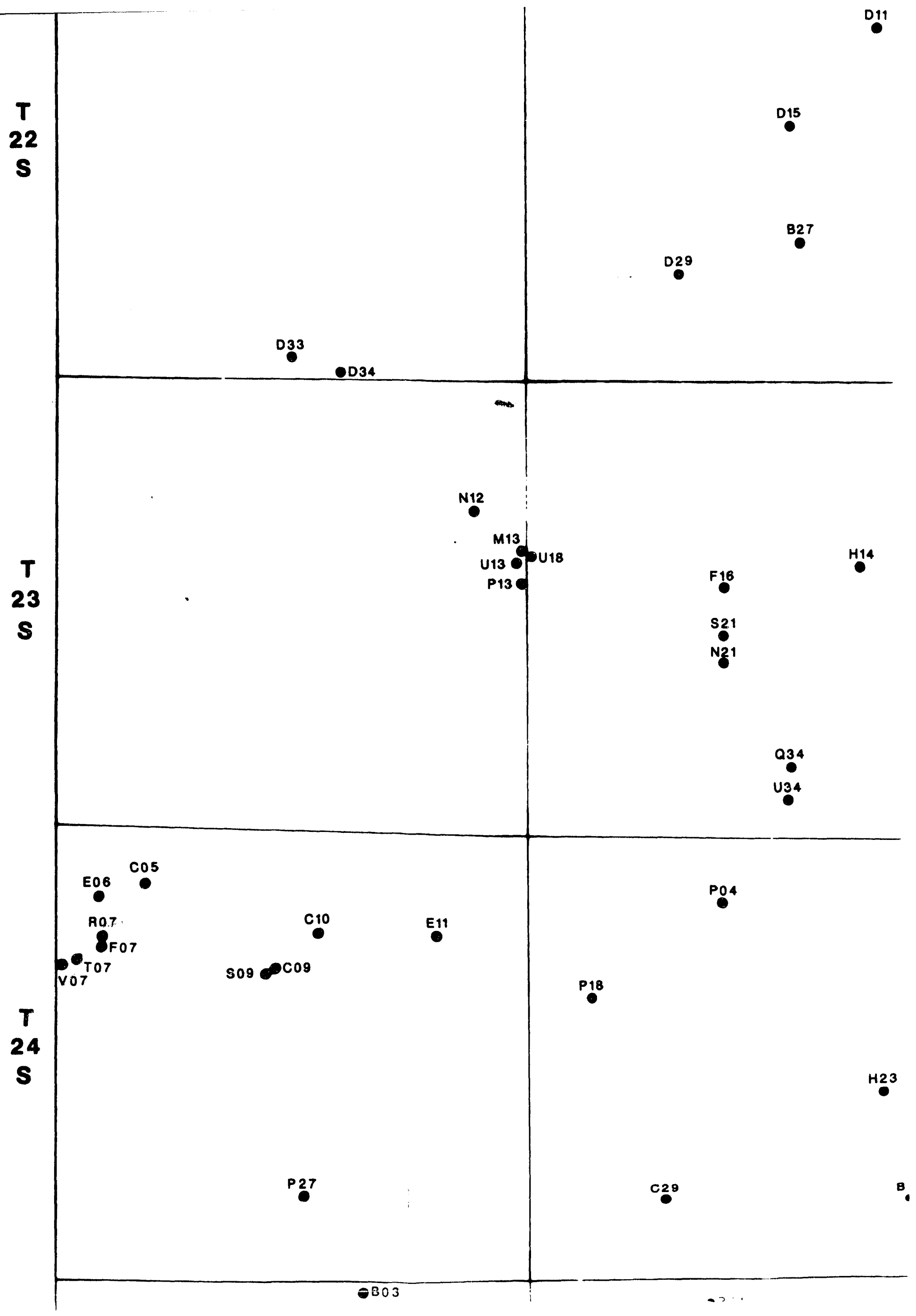




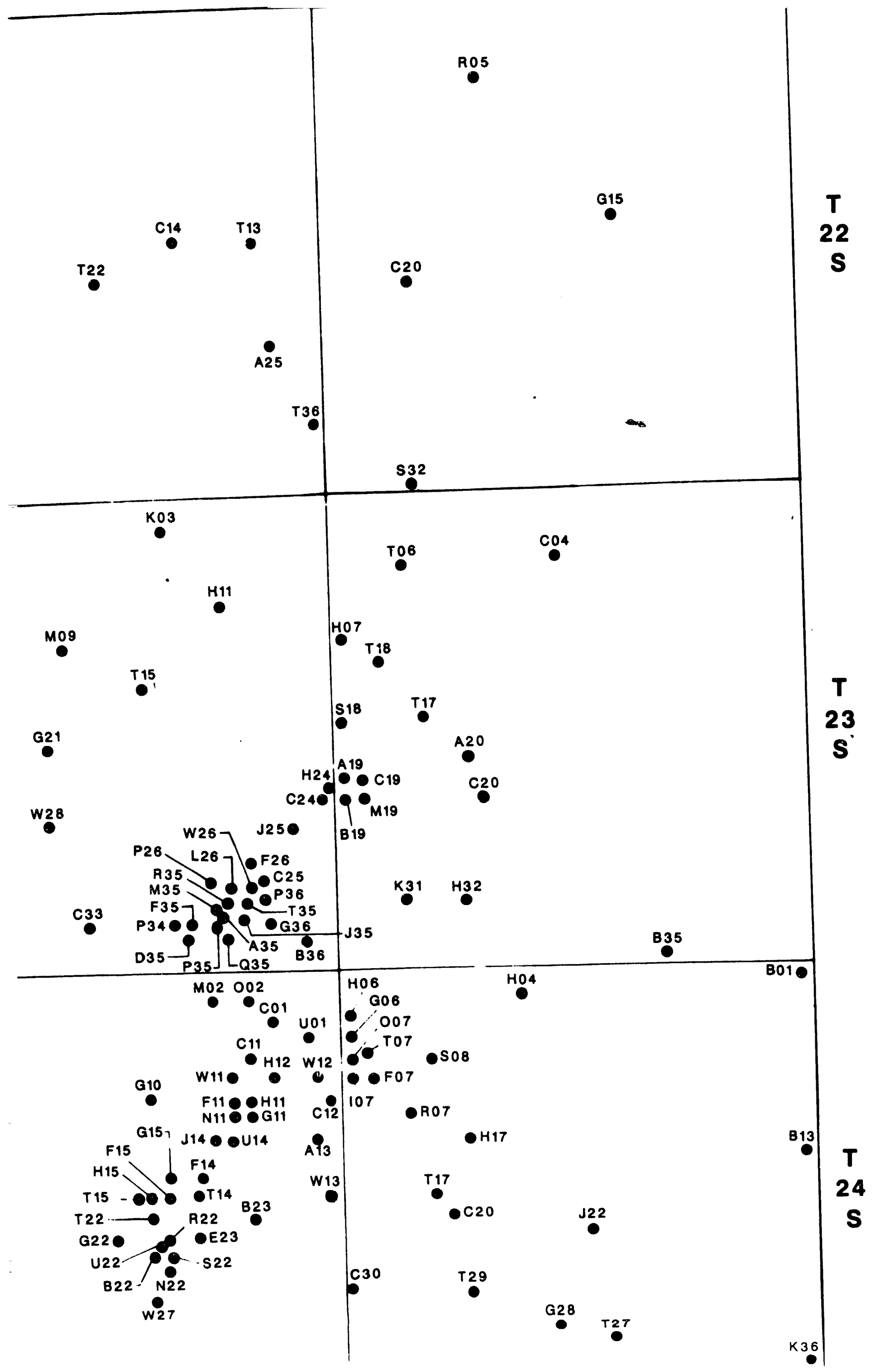




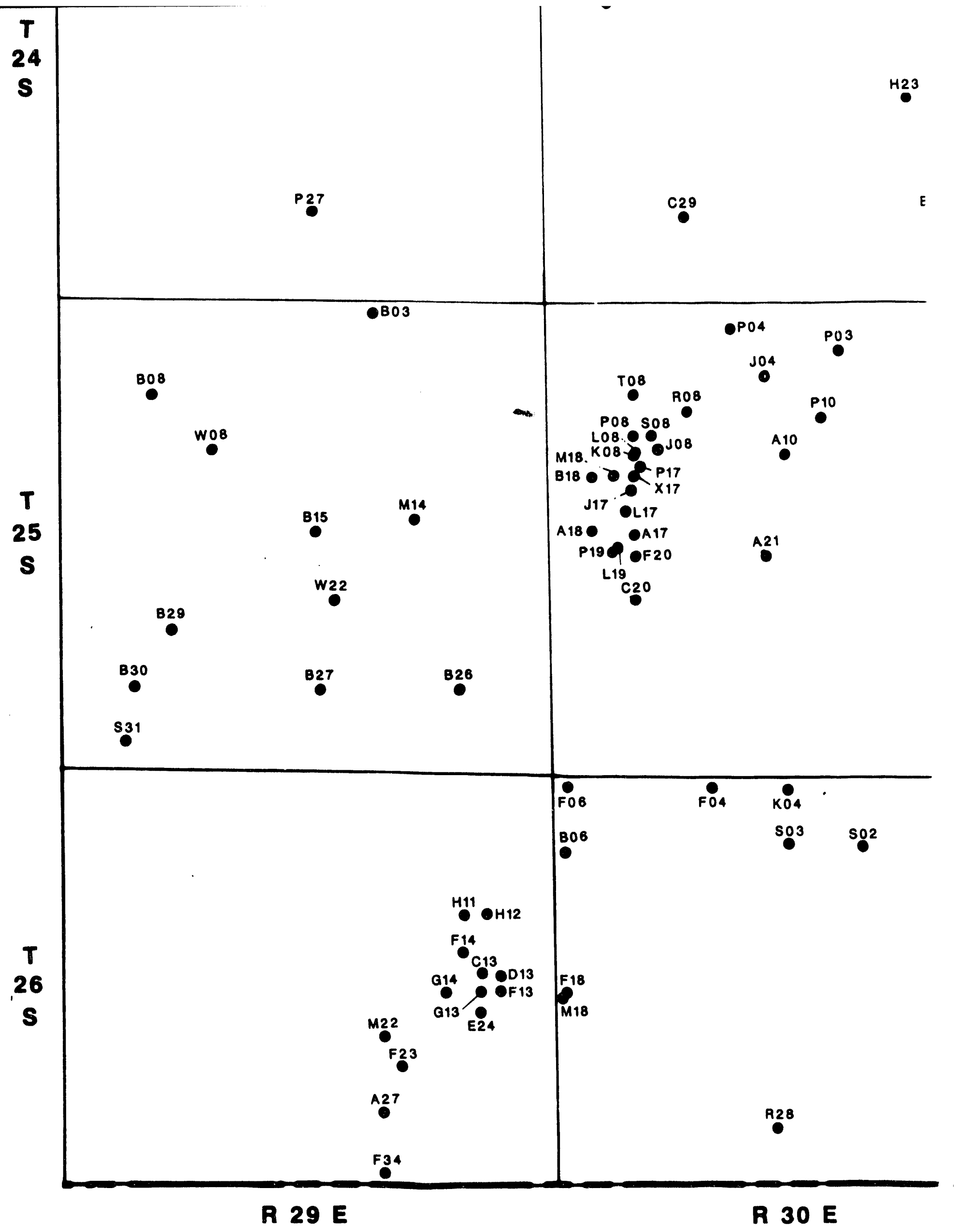




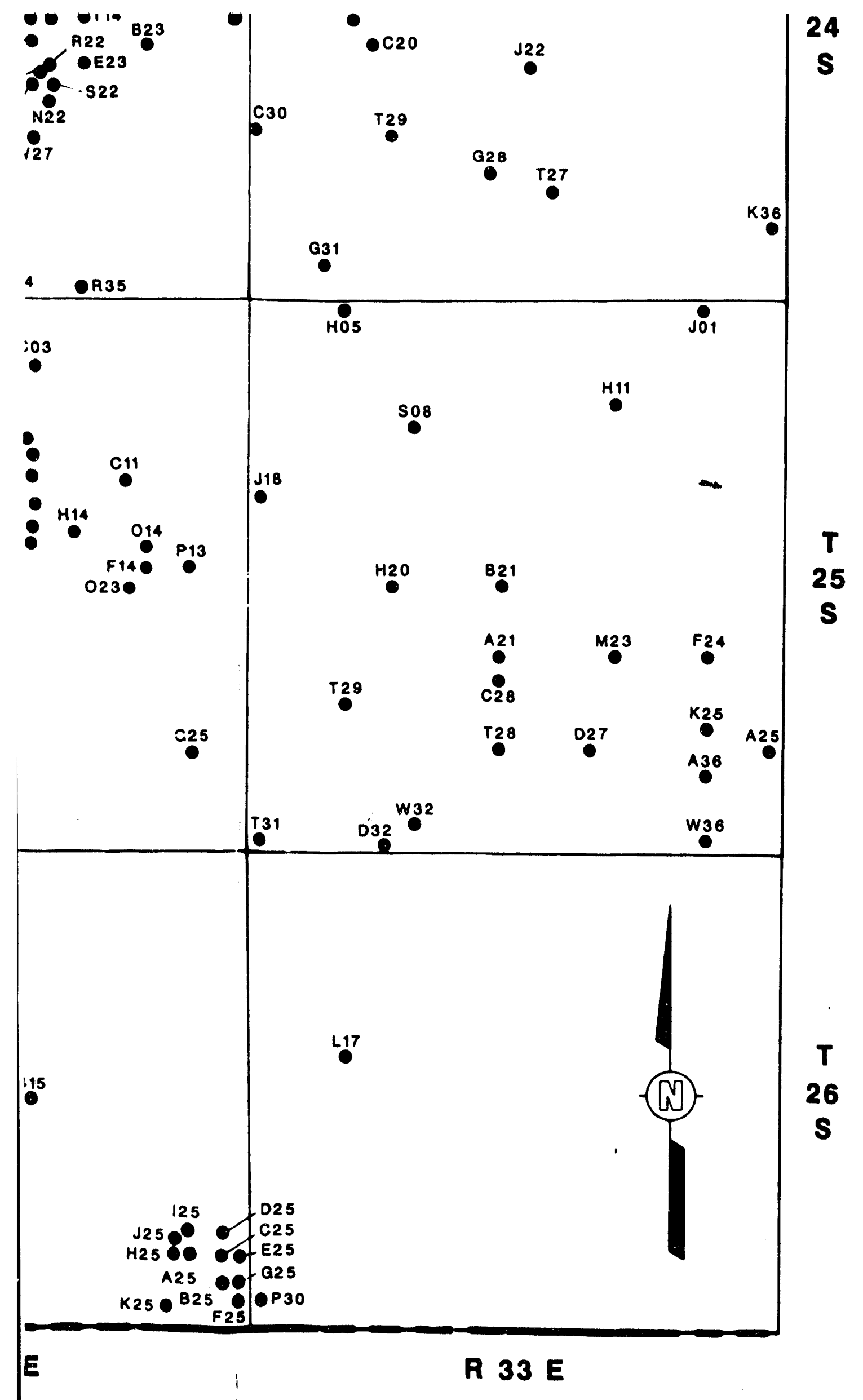

FIGURE 7.2-8 
Figure 7.2-9 Isopach Bottom of Vaca Triste to Bottom MB 123/124 


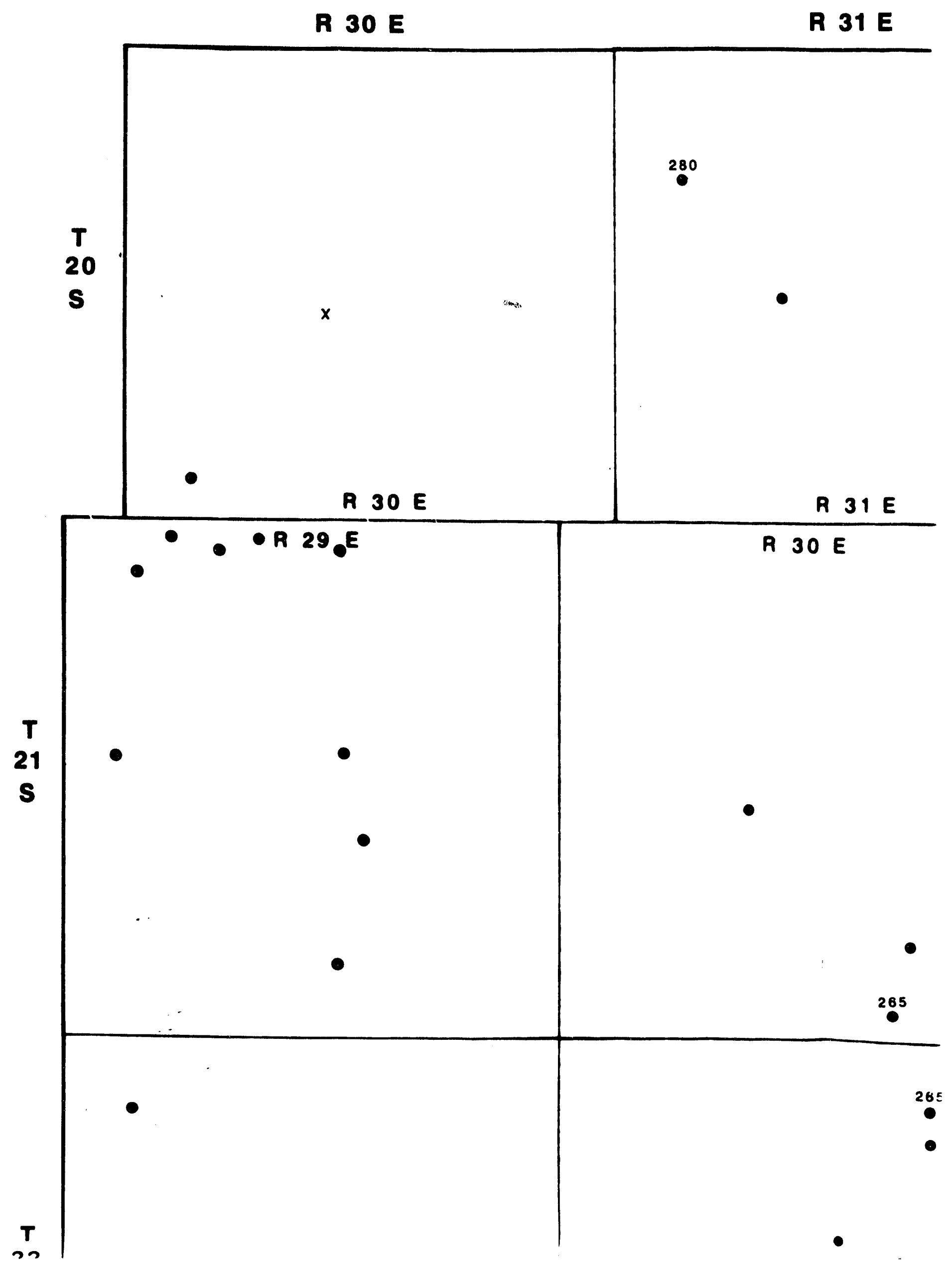




\section{R $31 \mathrm{E}$}

R $32 \mathrm{E}$

30

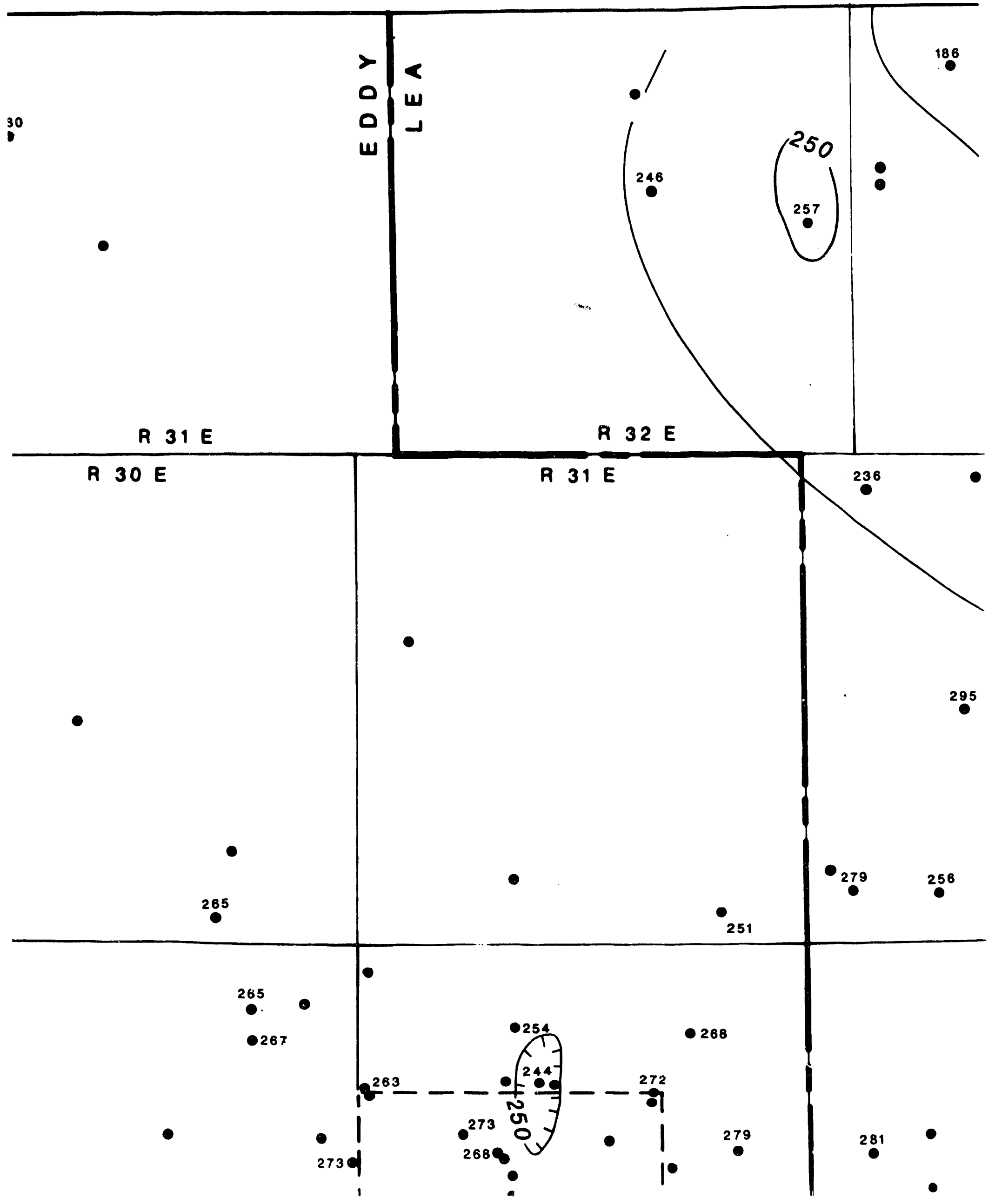


R. 33 E R 34 E

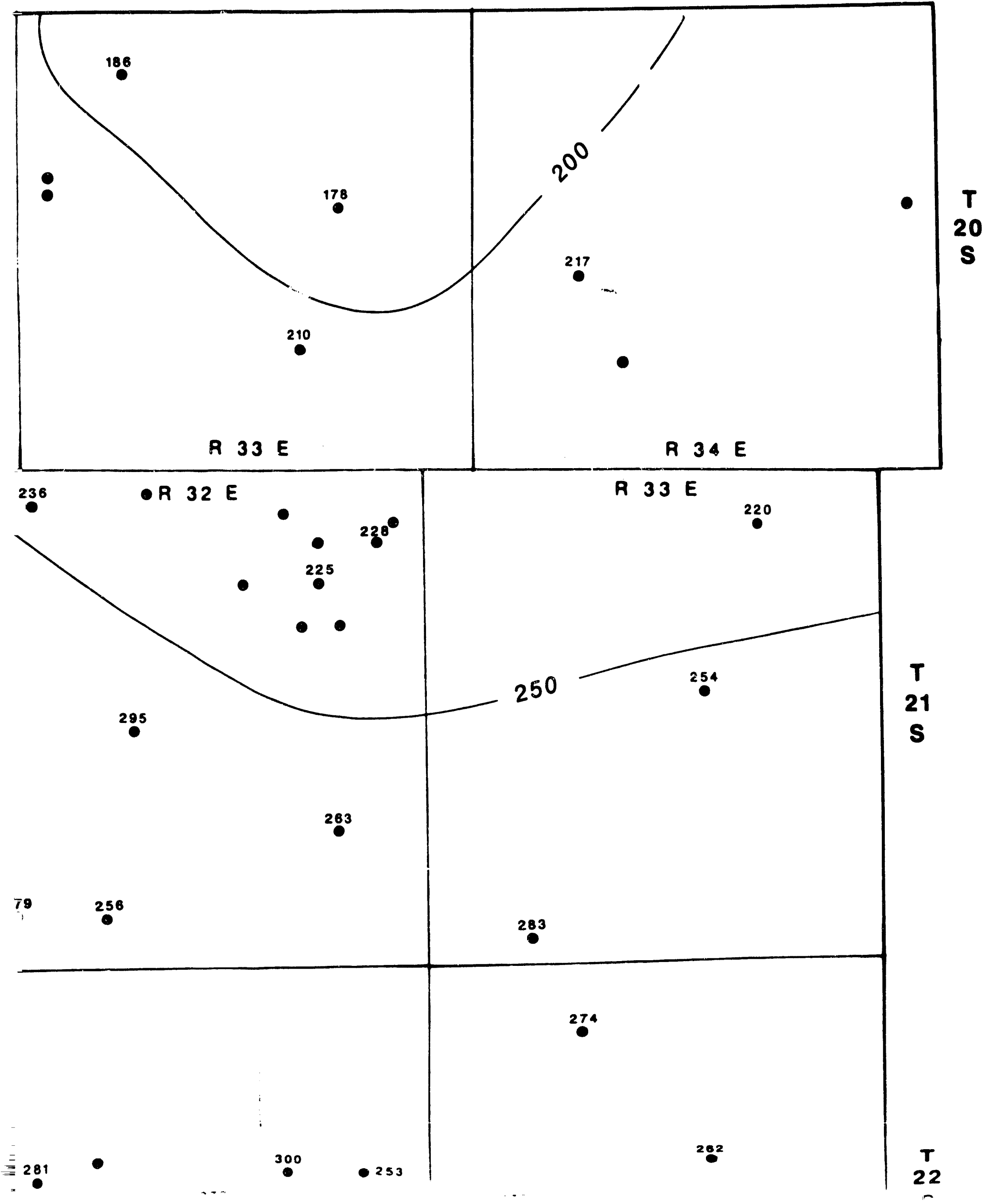




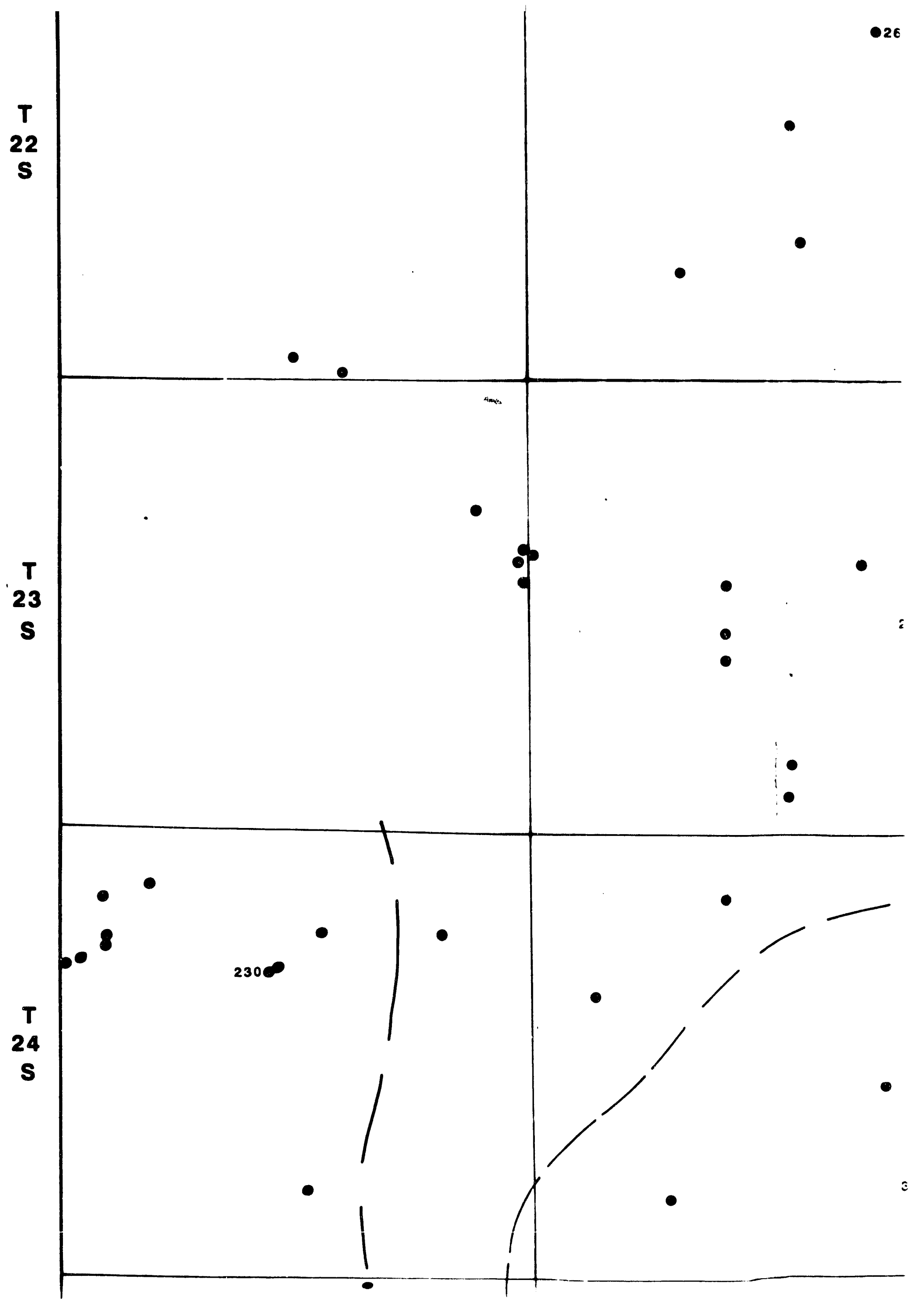




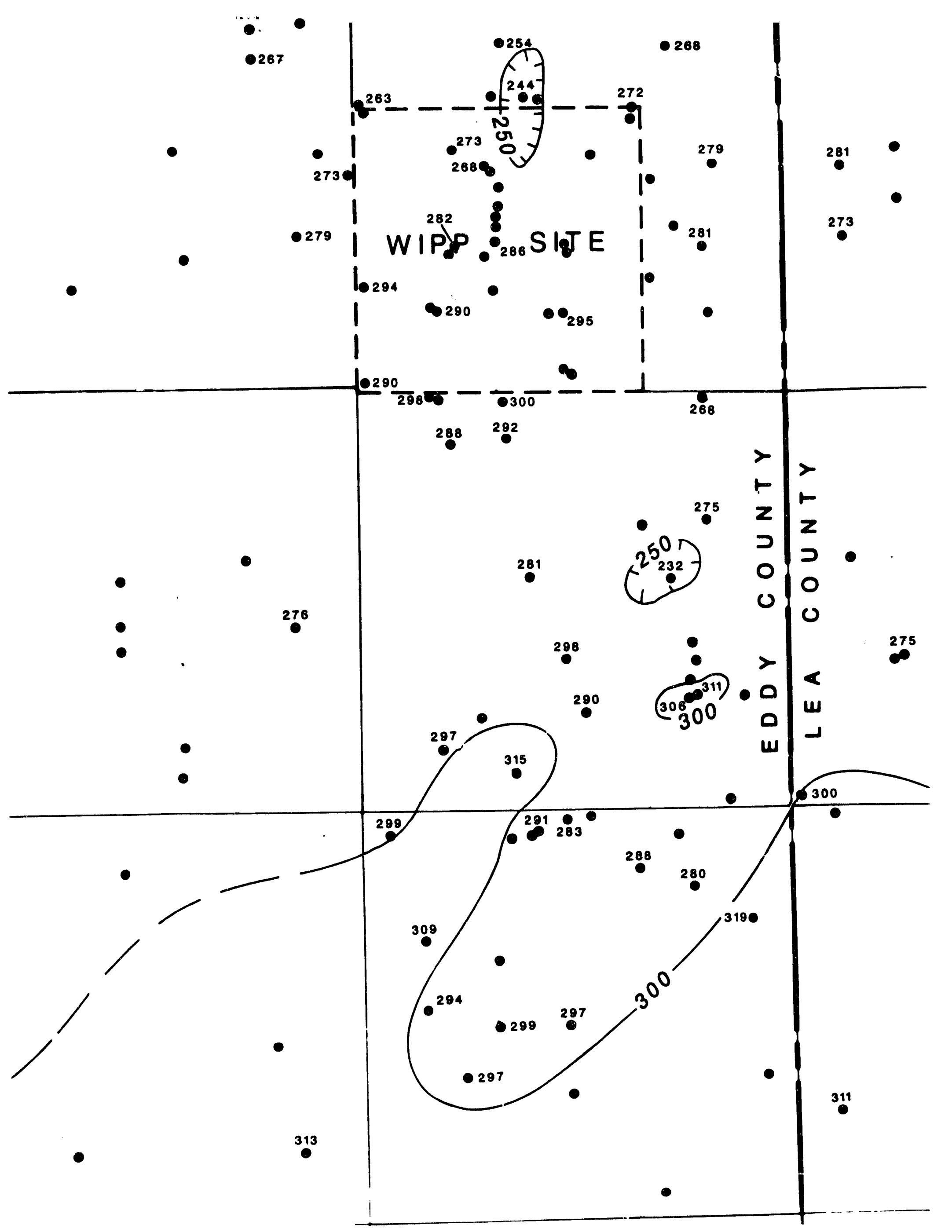




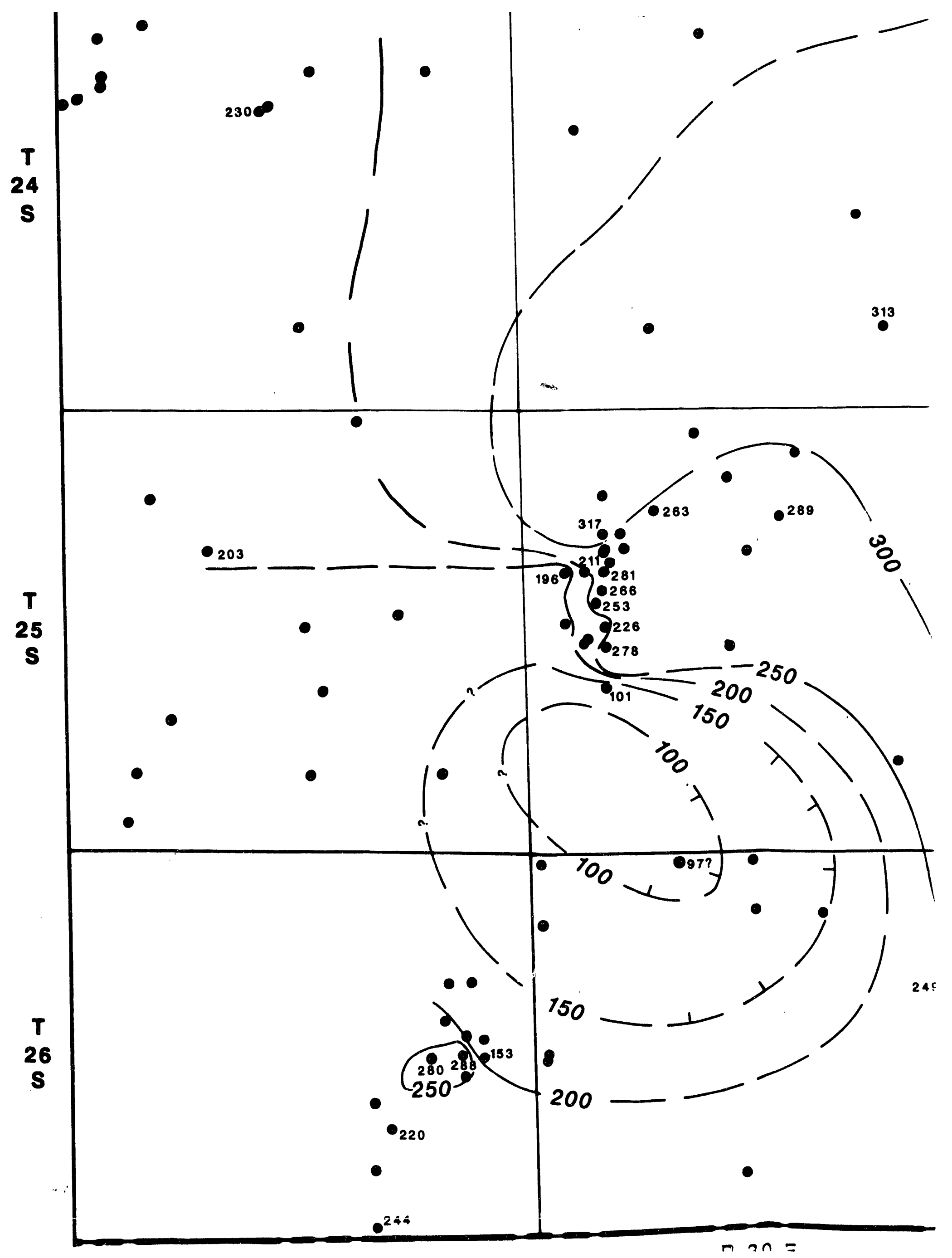




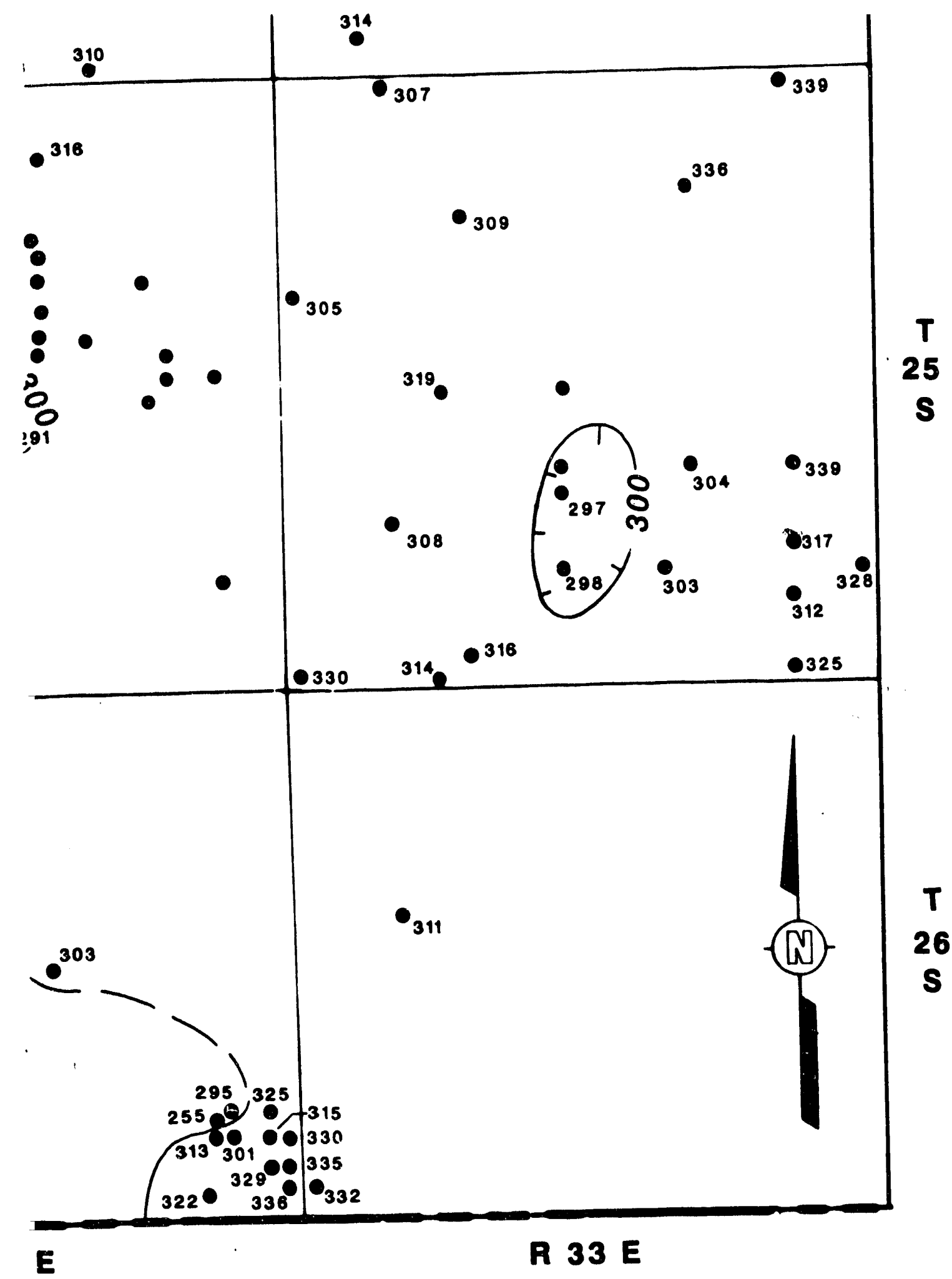

FIGURE 7.2-9

ISOPACH BOTTOM OF VACA TRISTE TO BOTTOM MB 123/124

(SEE FIGURE 7.2-8 FOR BOREHOLE IDENTIFIERS) 
Figure 7.2-10

Top of Salado to Top of Vaca Triste Isopach 
1
0
0
0
0
0
0
$\vdots$
0
0
0

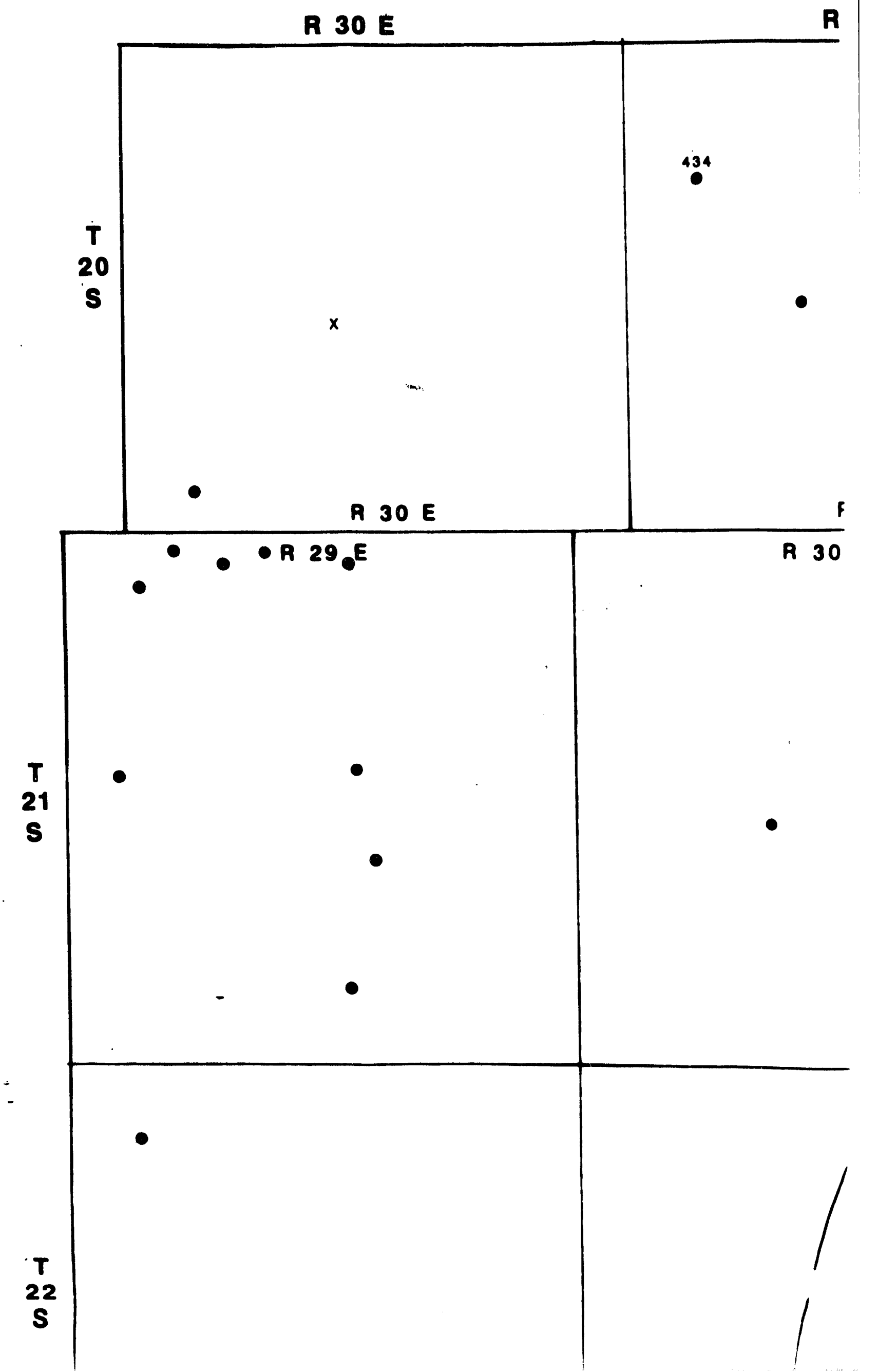




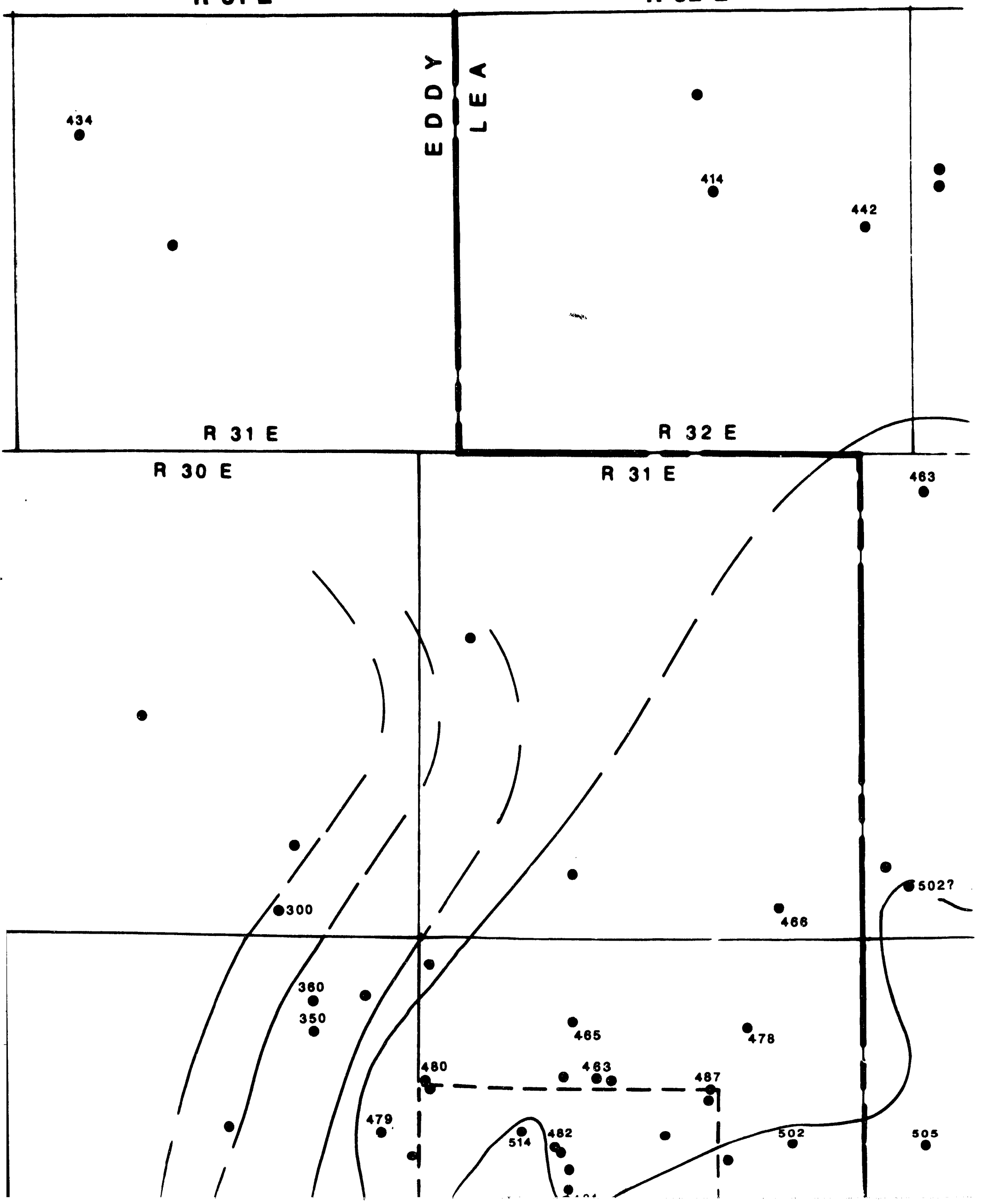




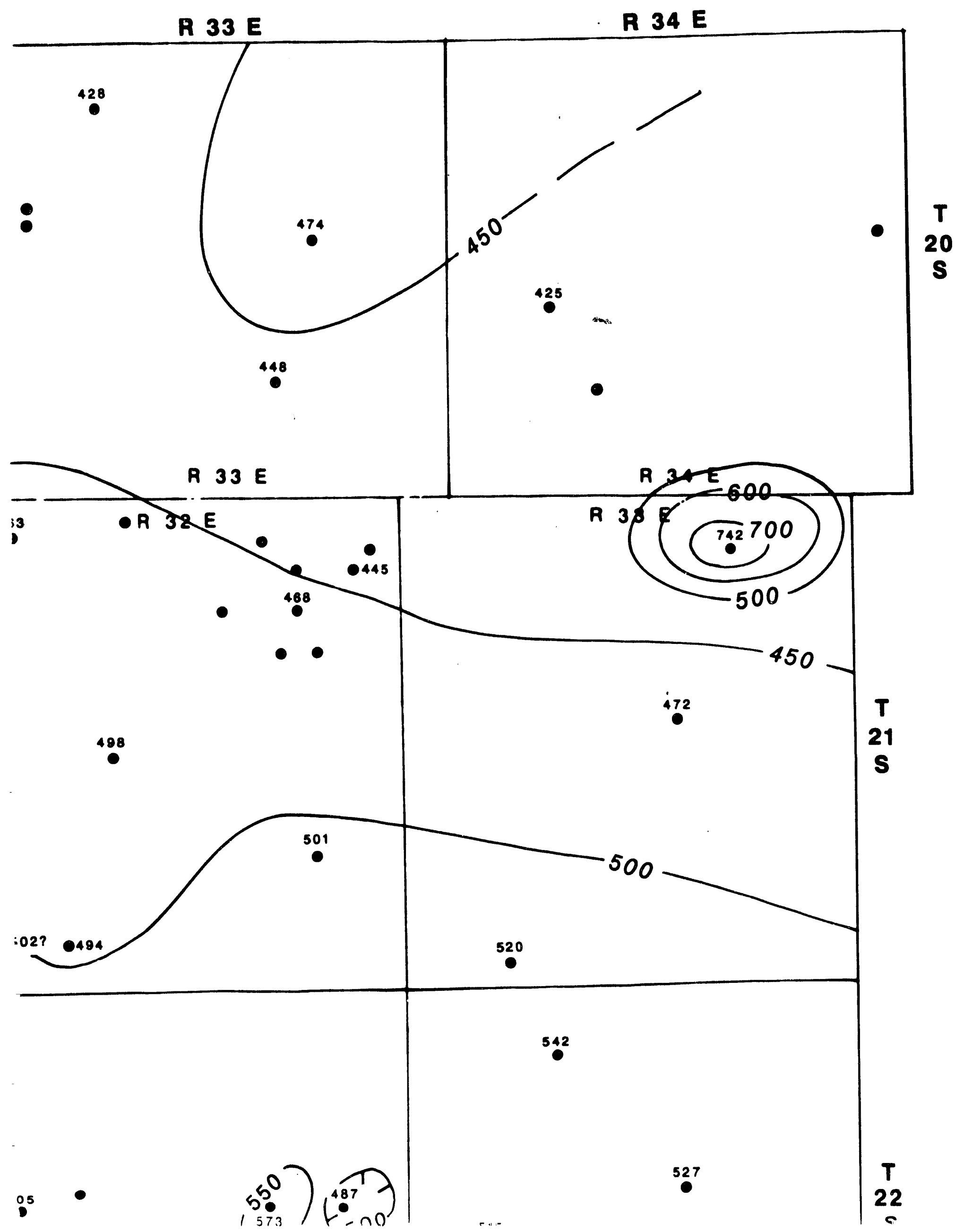




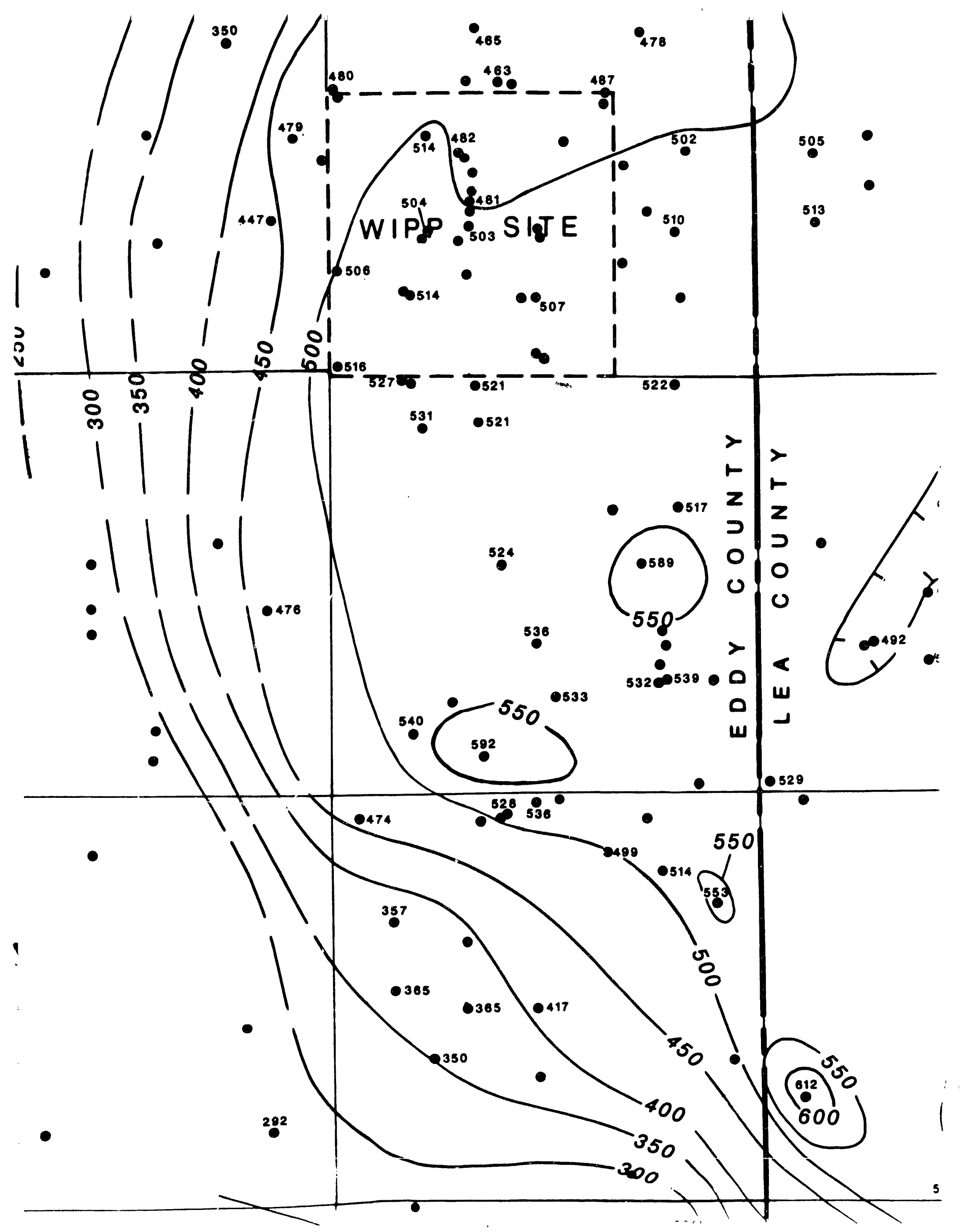




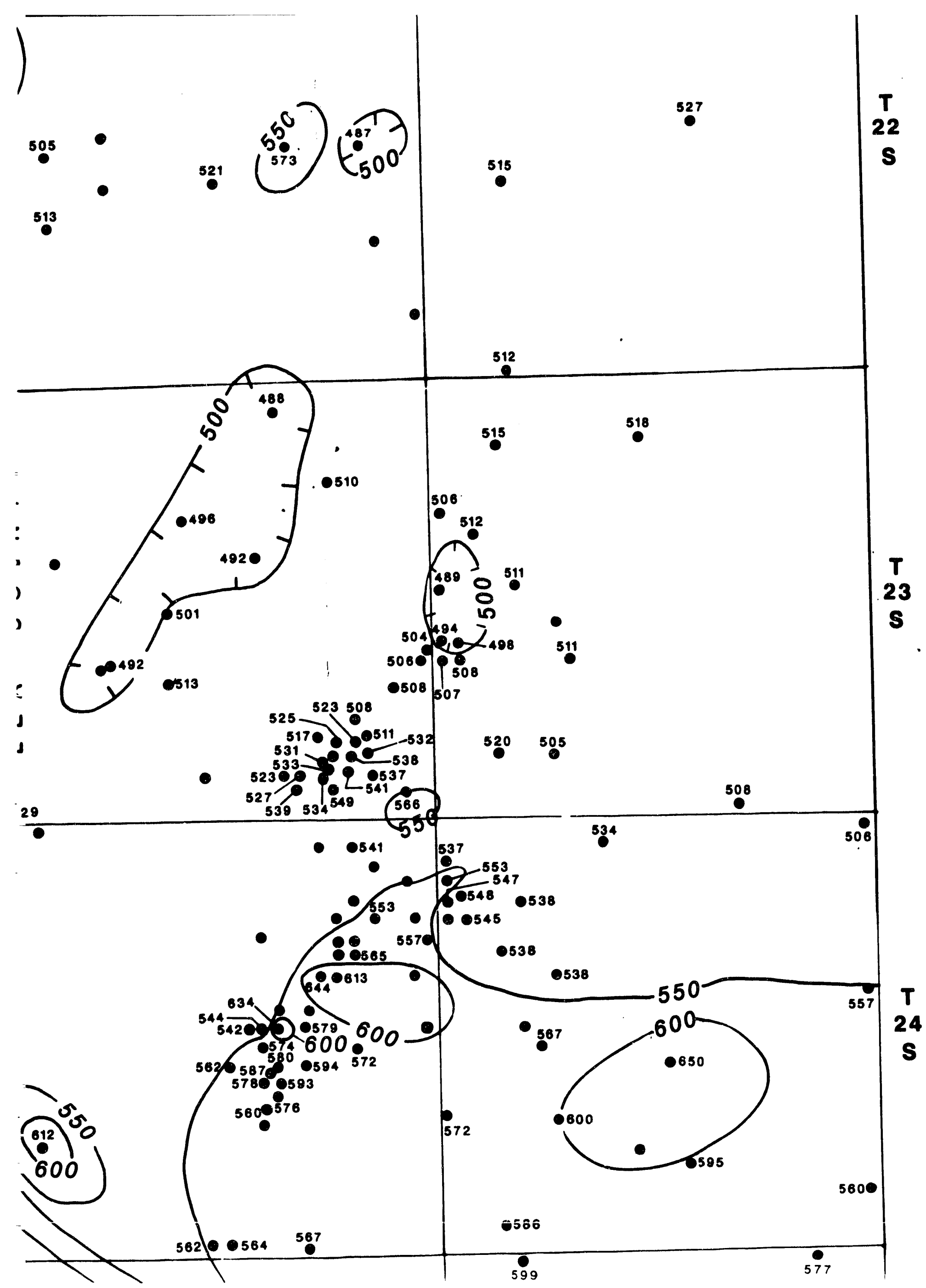




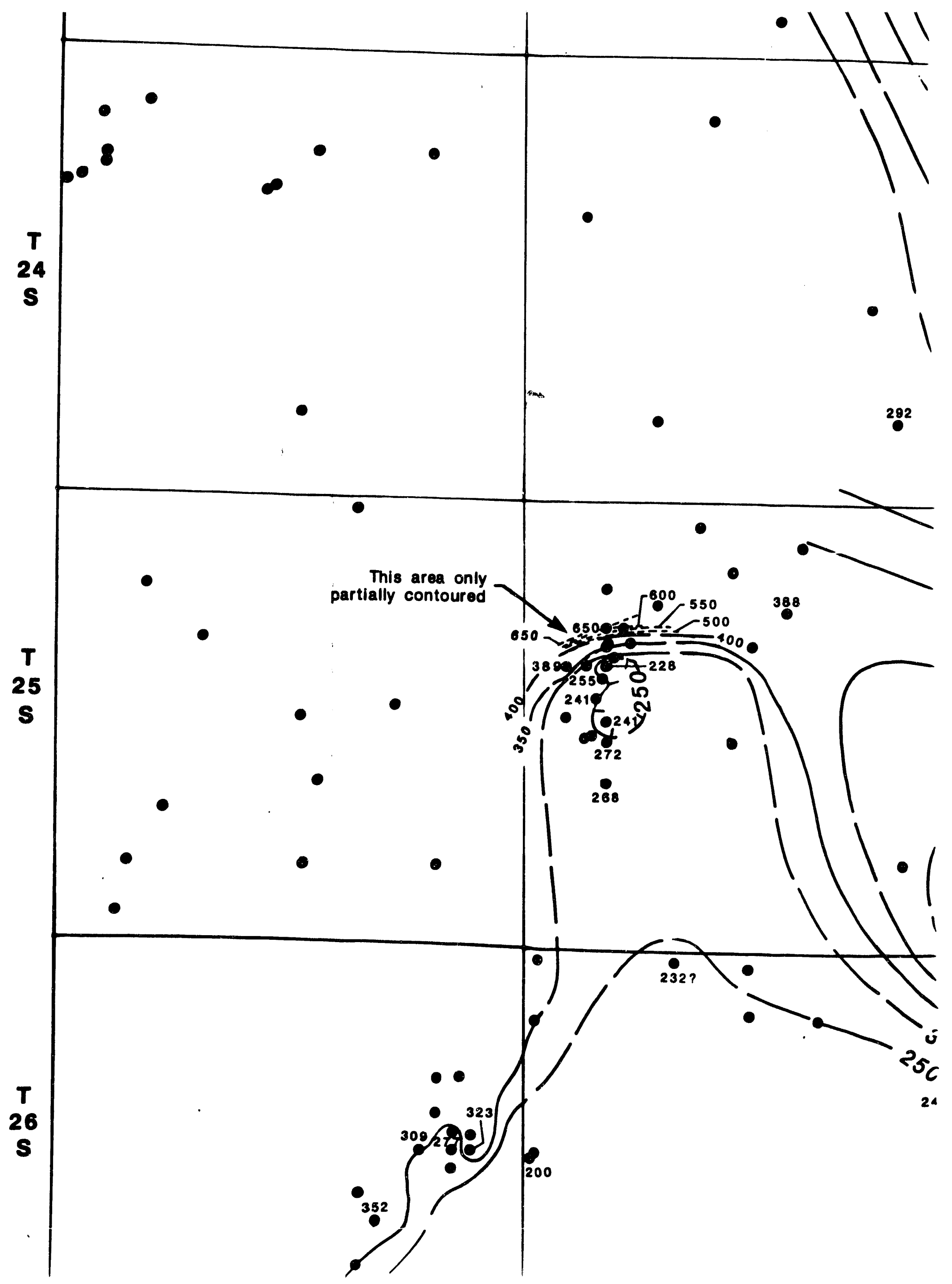




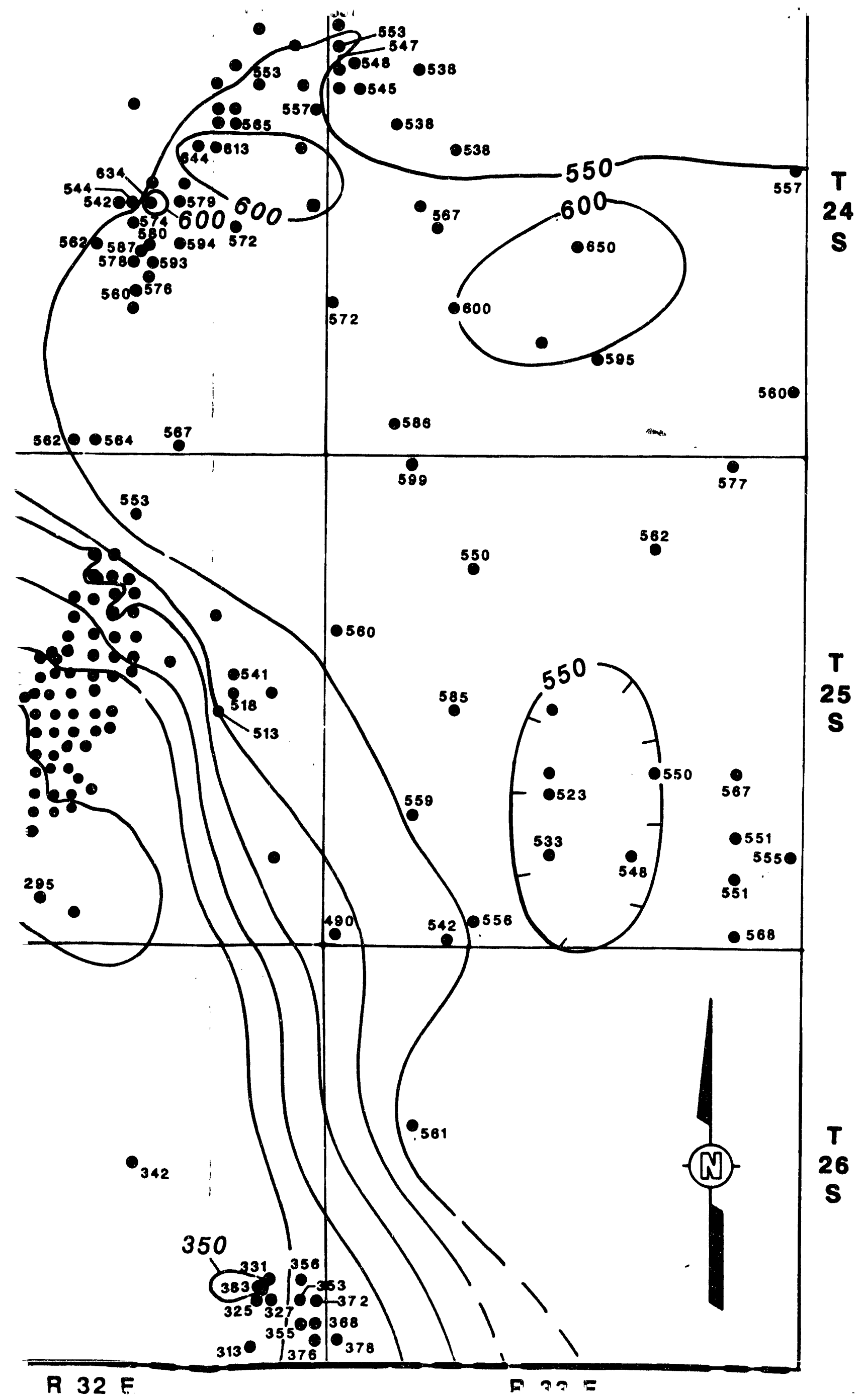




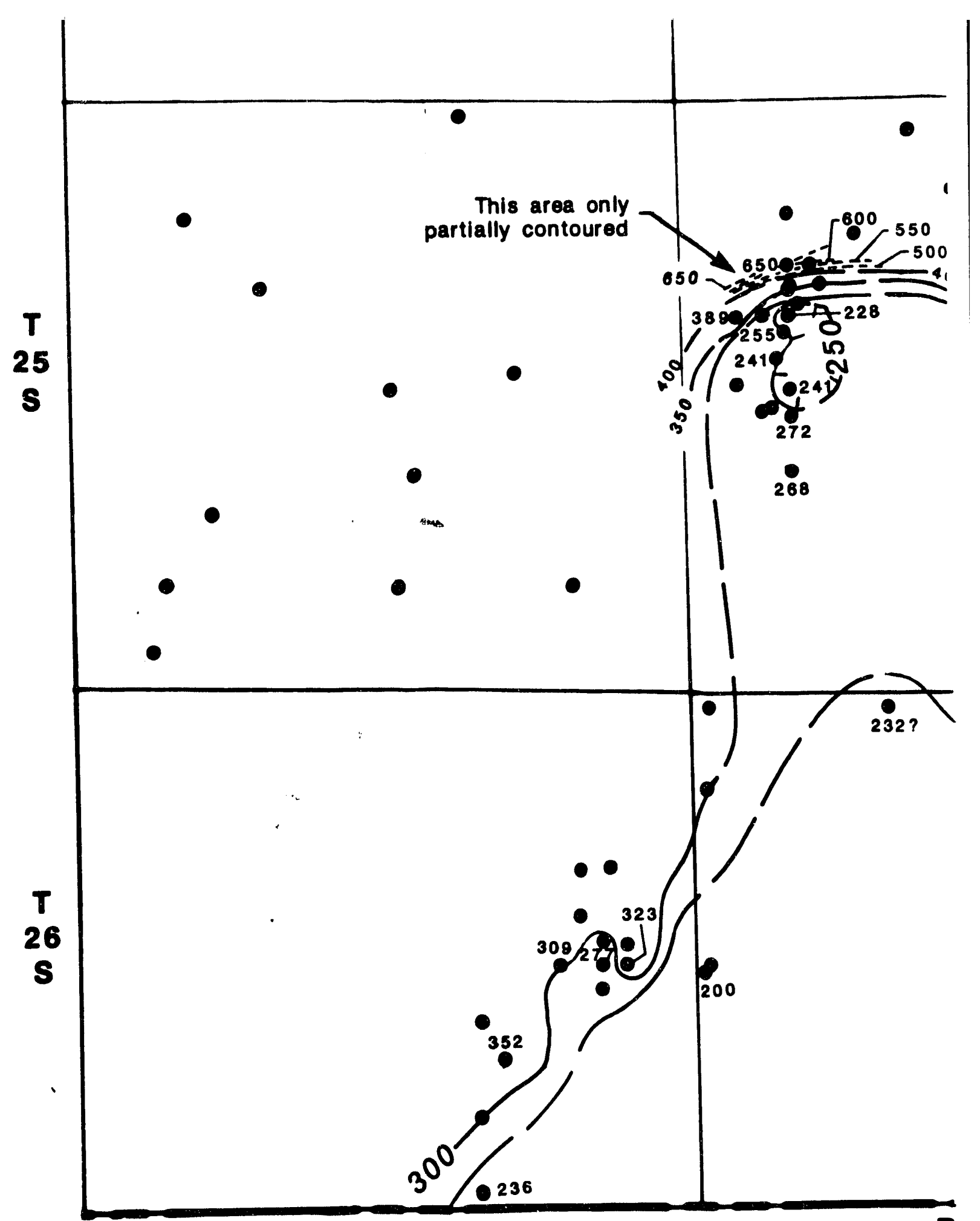

R $29 \mathrm{E}$

CONTOUR INTERVAL-5O FT - DASHED CONTOURS REPRESENT AREAS OF
LIMITED STRATIGRAPHIC CONTROL 


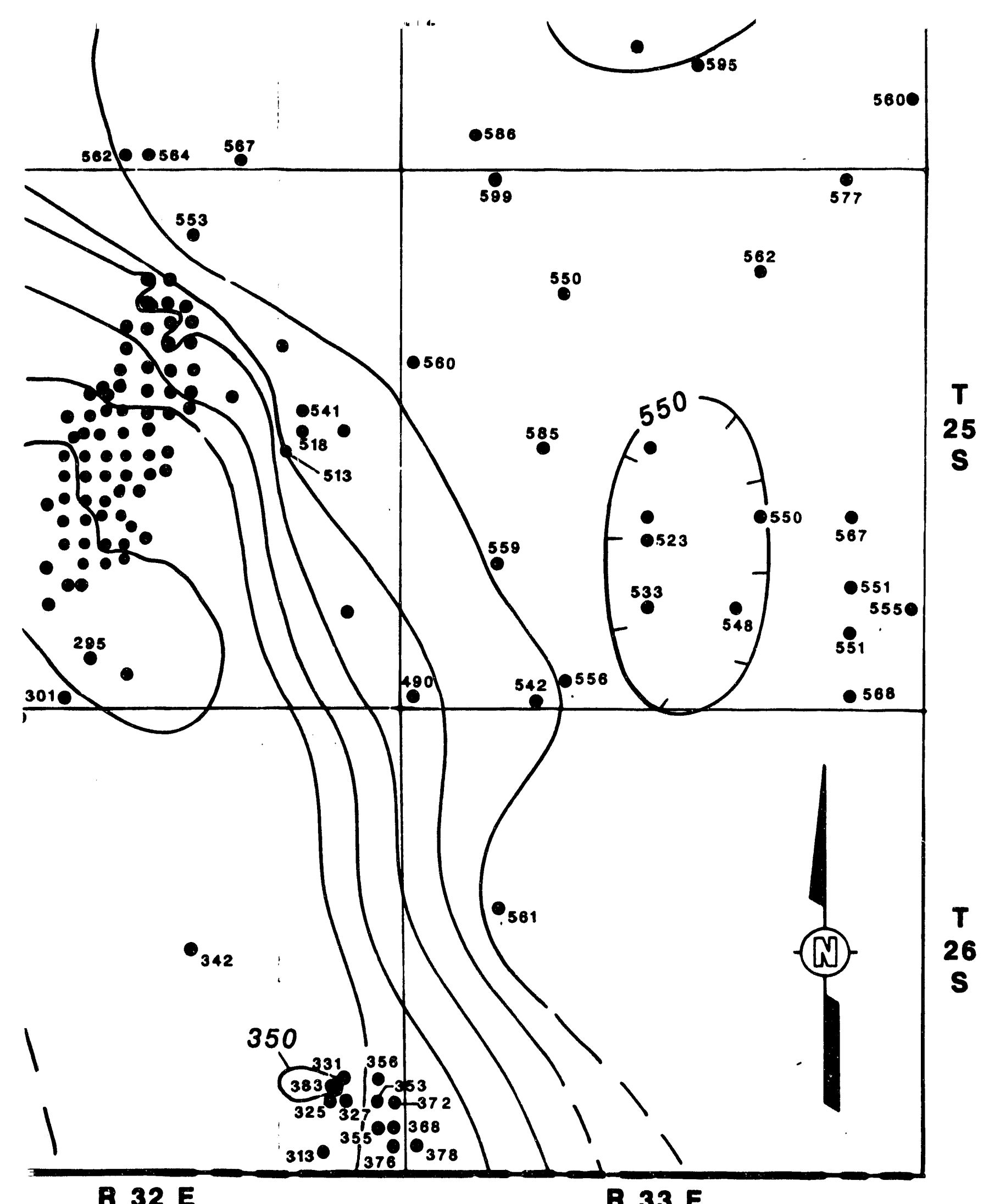

R 32 E

R 33 E

FIGURE 7.2-10

TOP OF SALADO TO TOP OF

VACA TRISTE ISOPACH

(SEE FIGURE 7.2-8 FOR BOREHOLE IDENTIFIERS) 
Figure 7.2-11

Isopach Bottom Union - Top MB123 


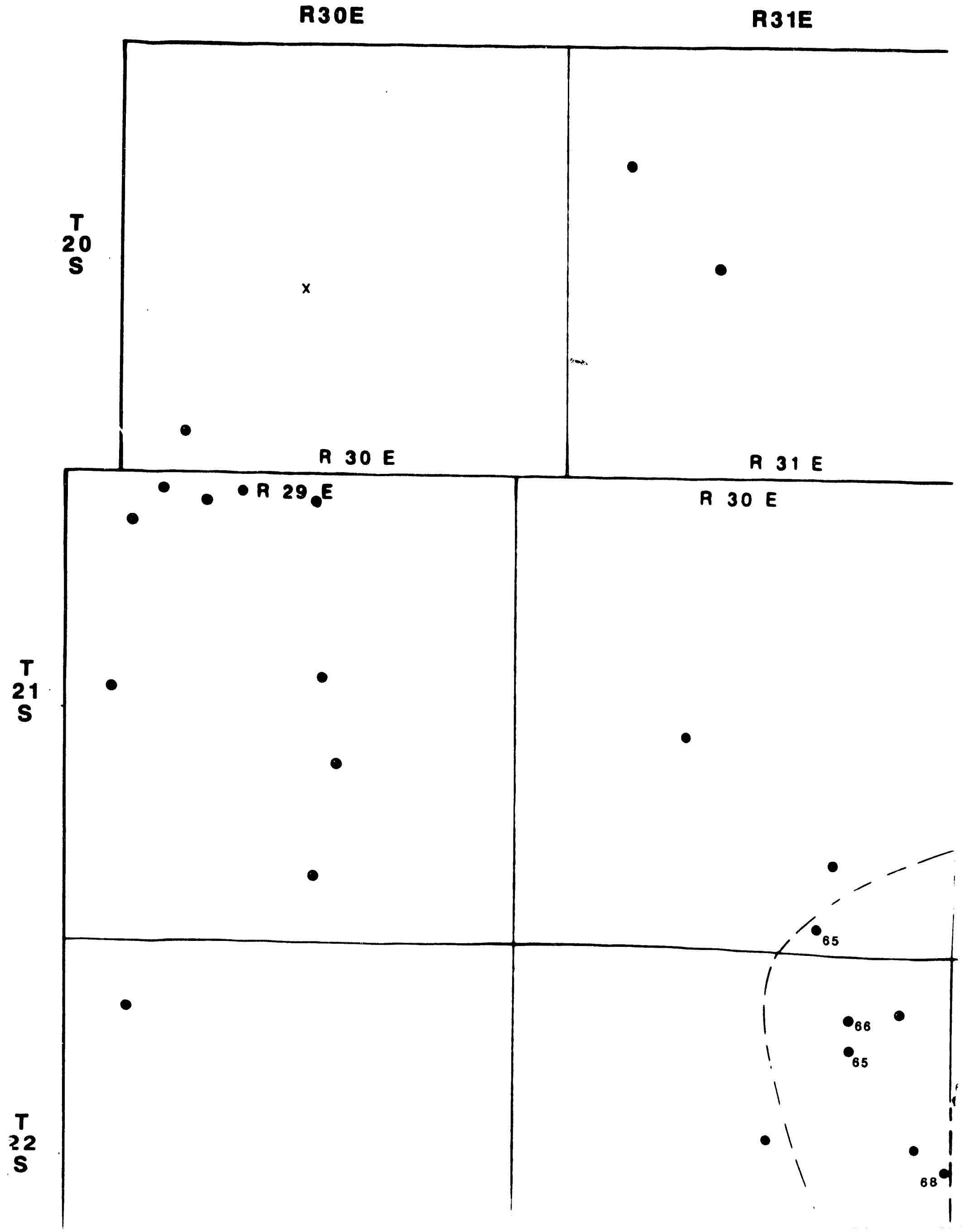




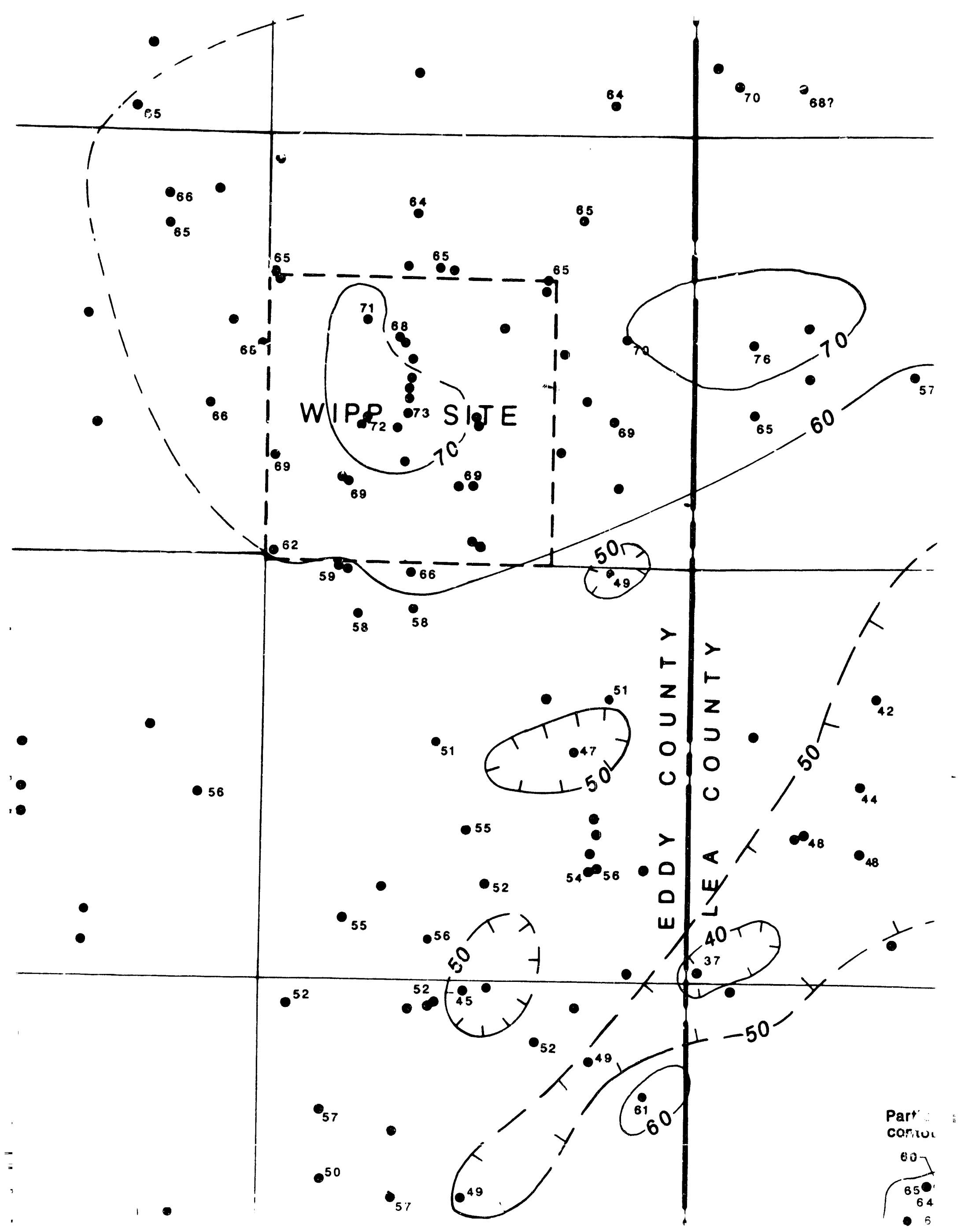




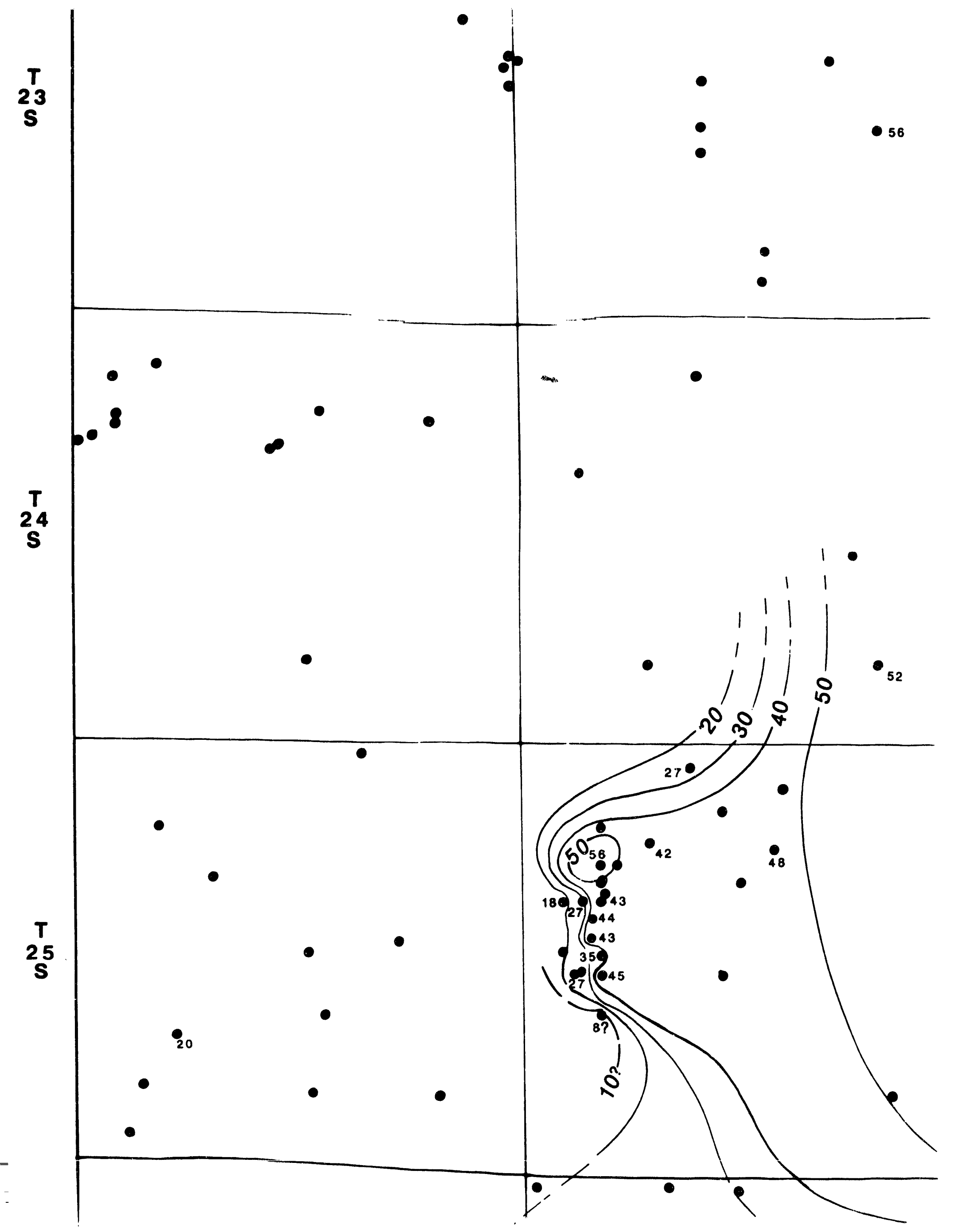




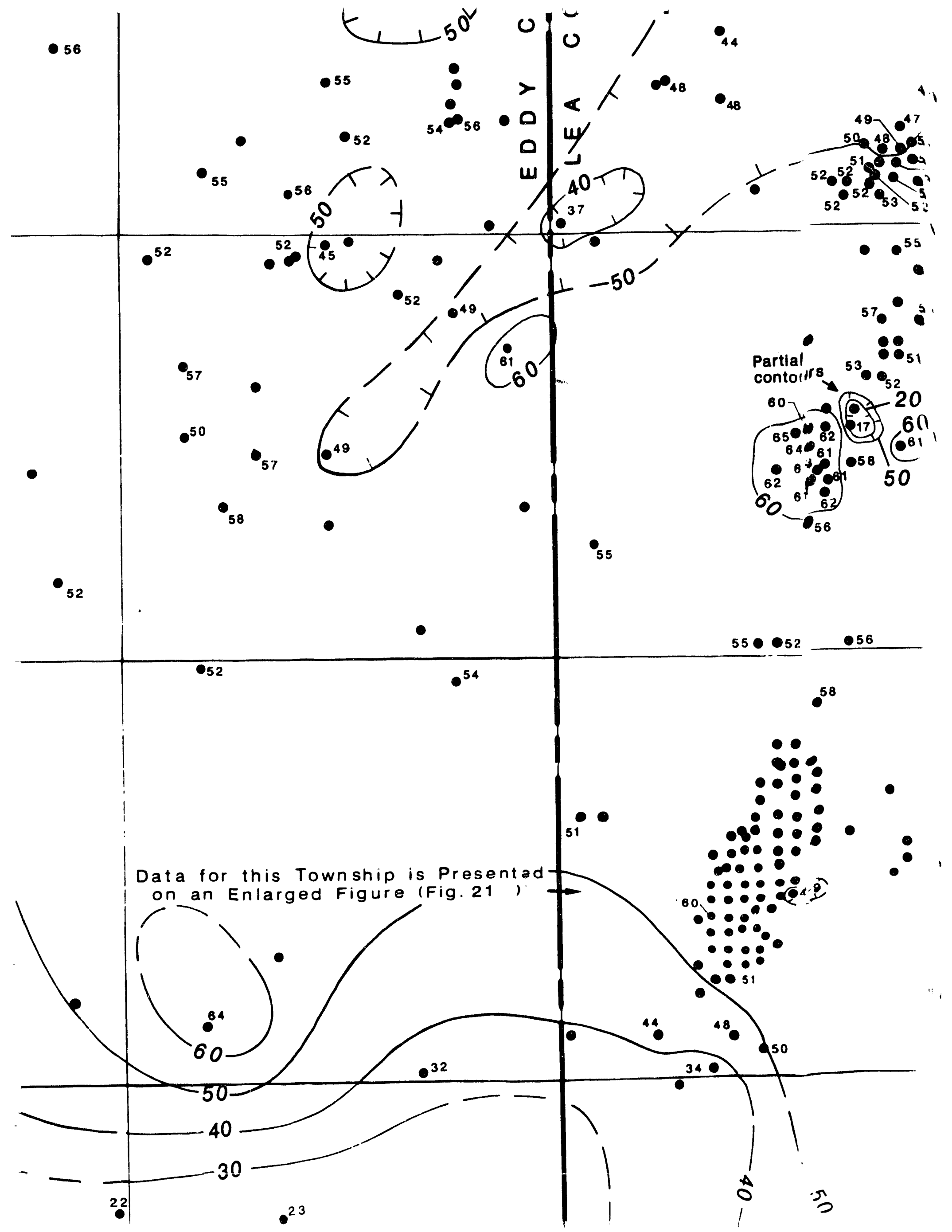




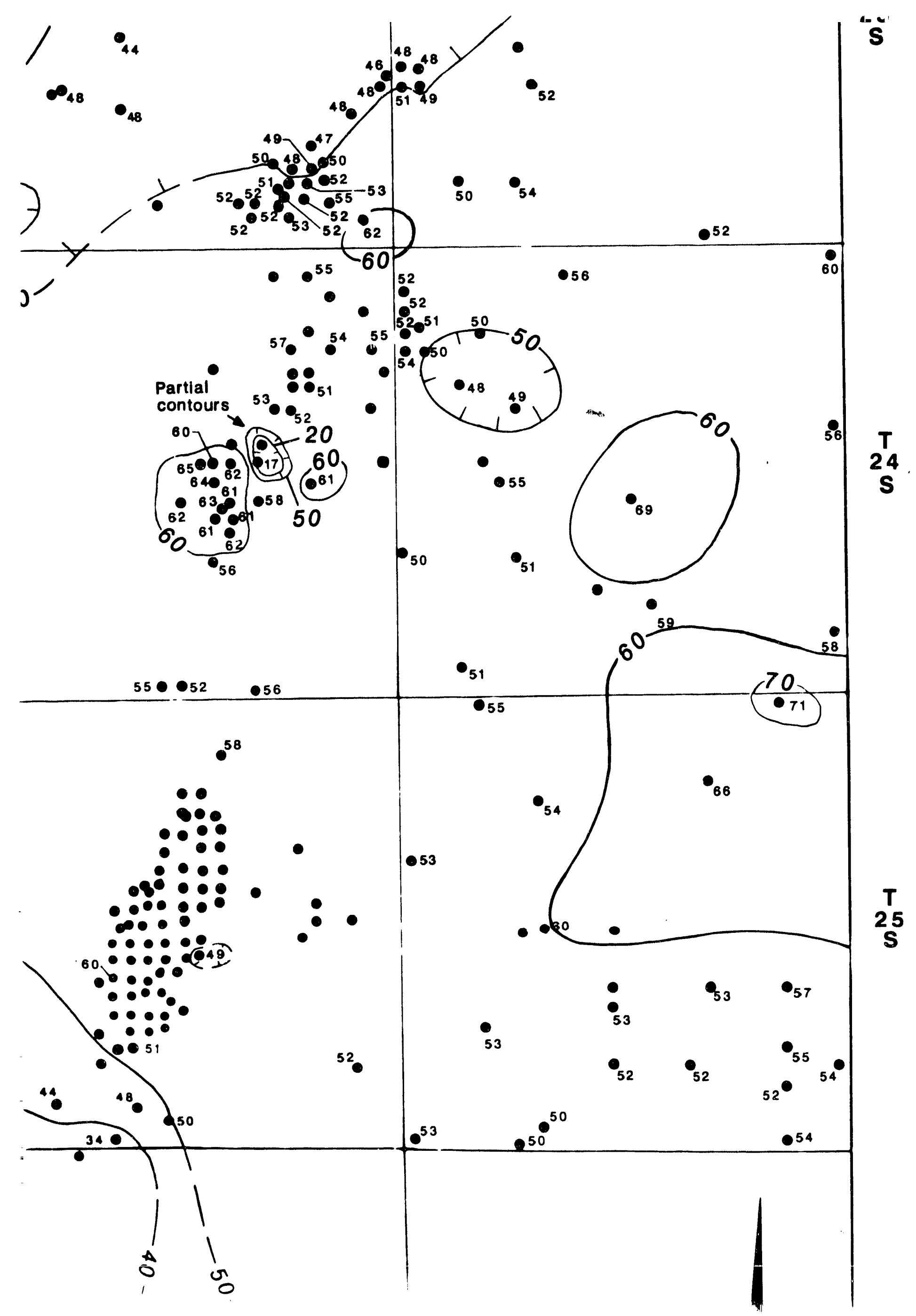




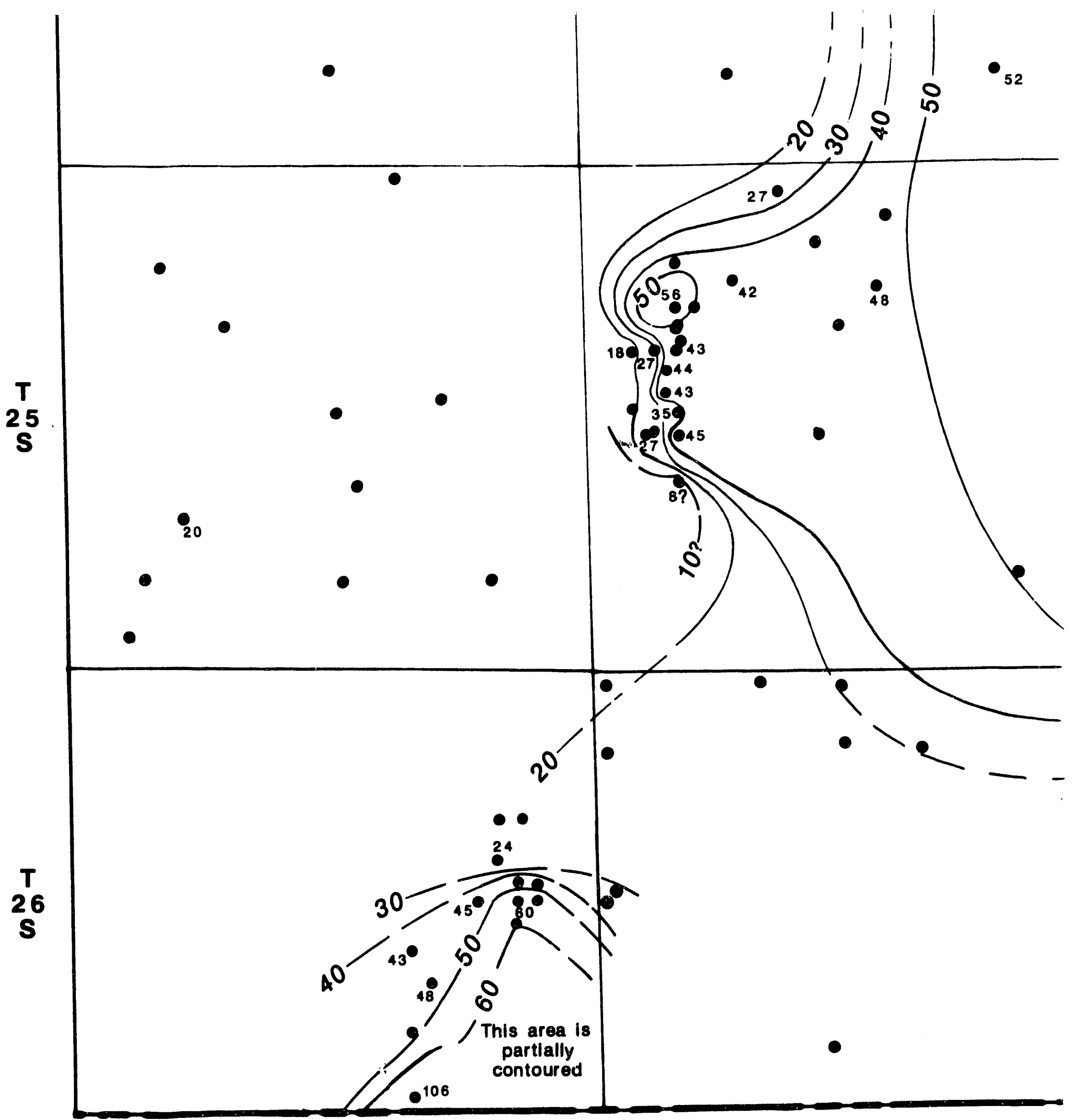

R29E

R3 OE

CONTOUR INTERVAL=10 FT

DASHED CONTOURS REPRESENT
AREAS OF LIMITED STRATIGRAPHIC CONTROL 


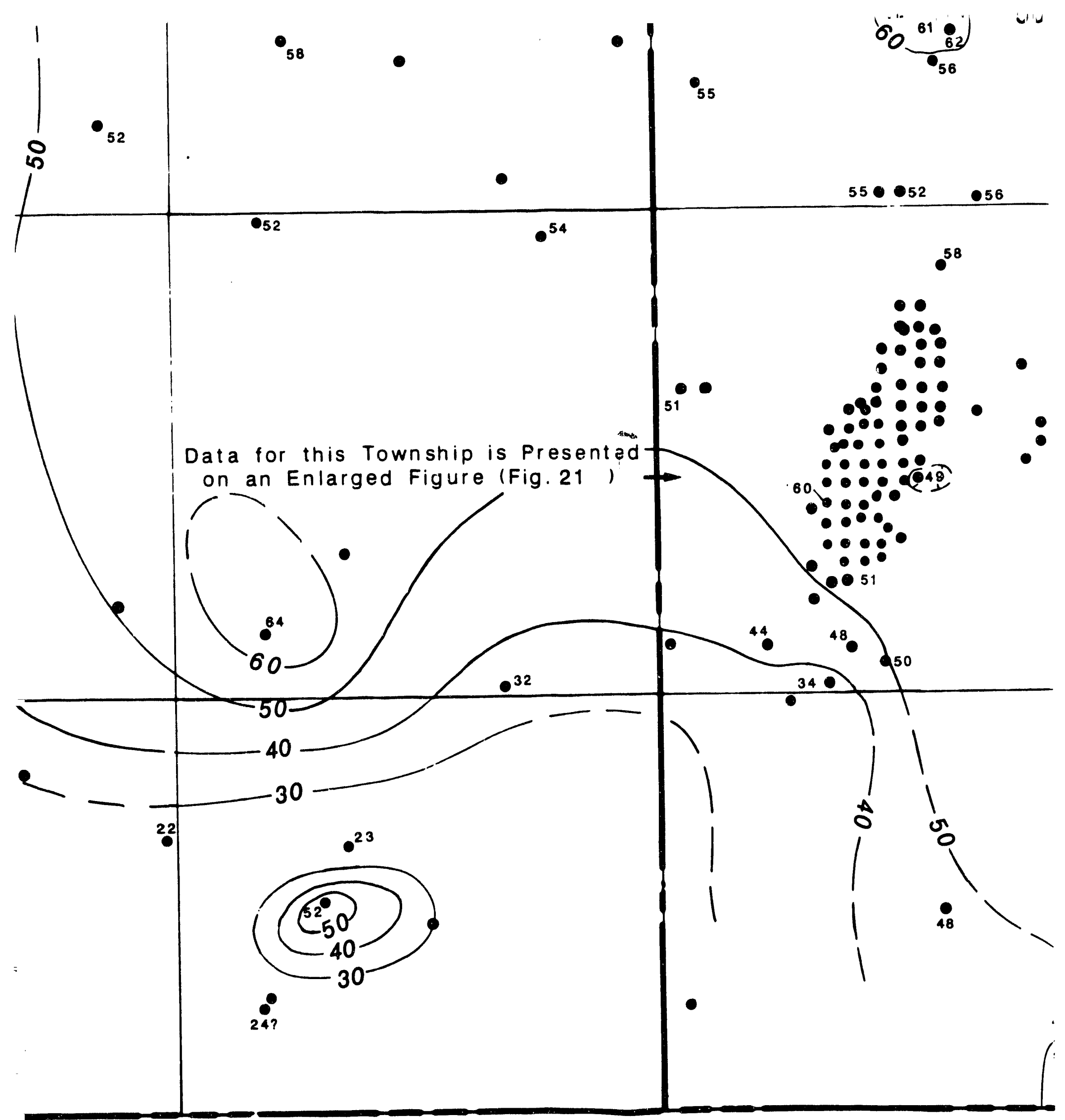

R31E

R32E 
Figure 7.2-12

Isopach MB109 Base to Top VT 


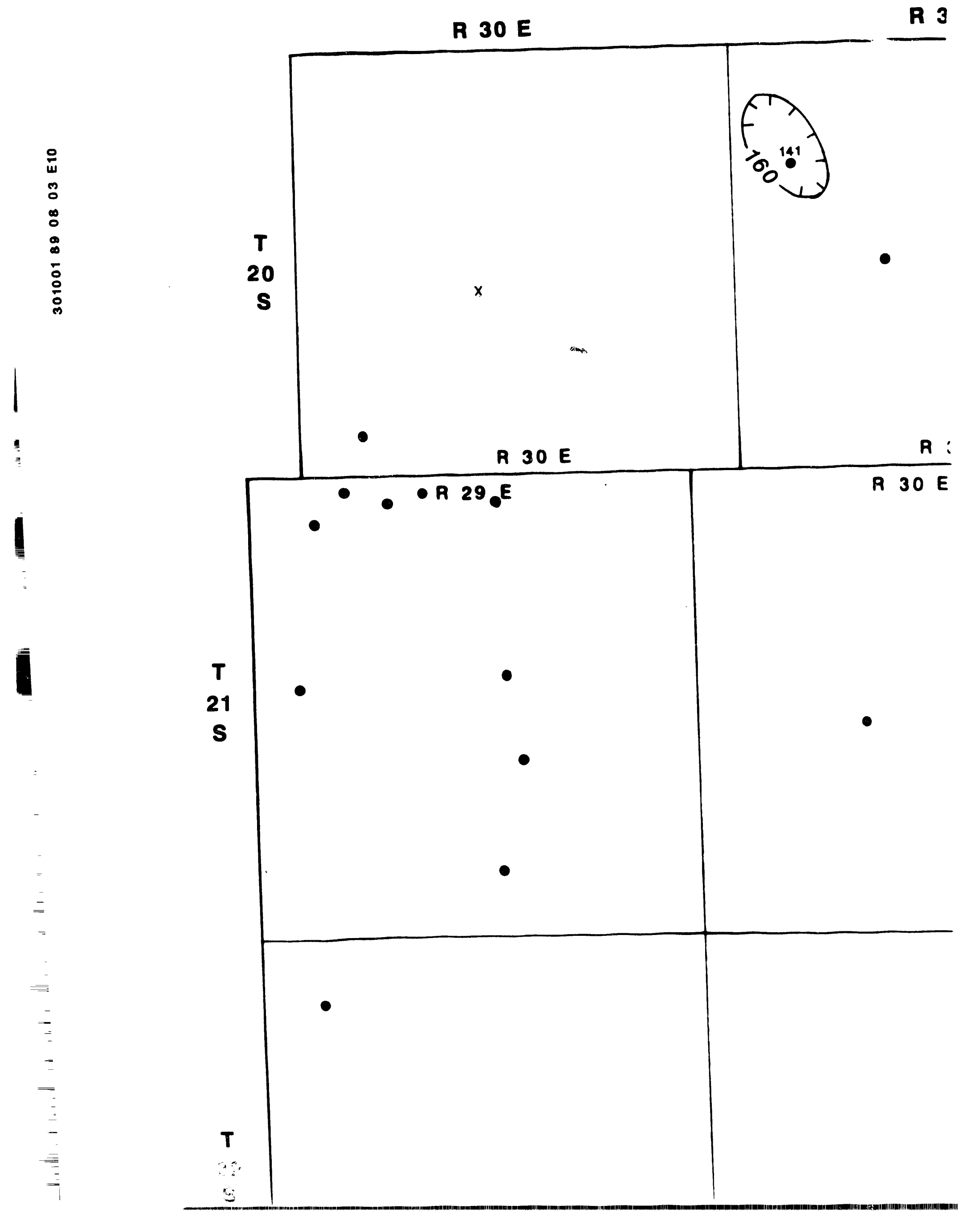




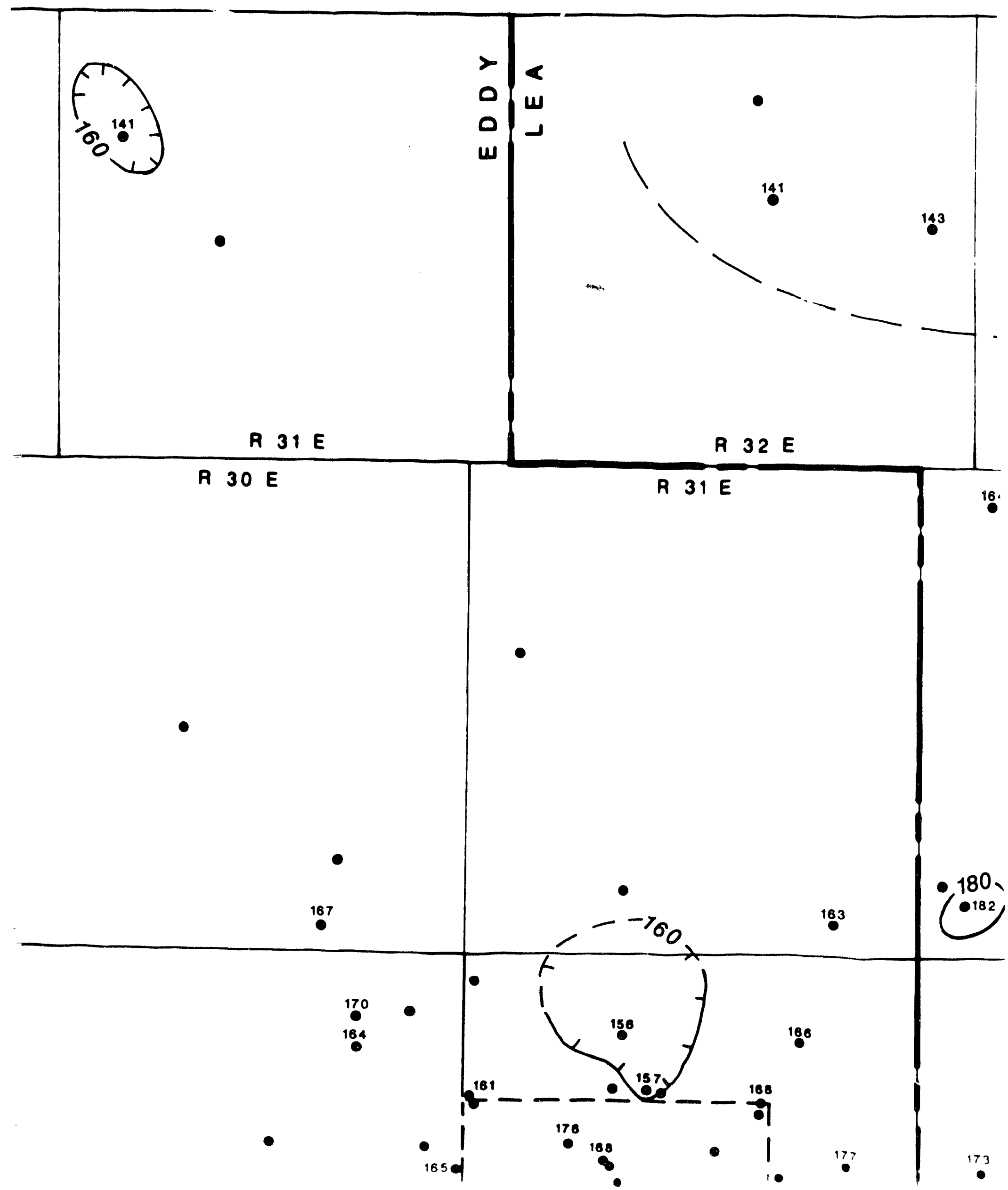




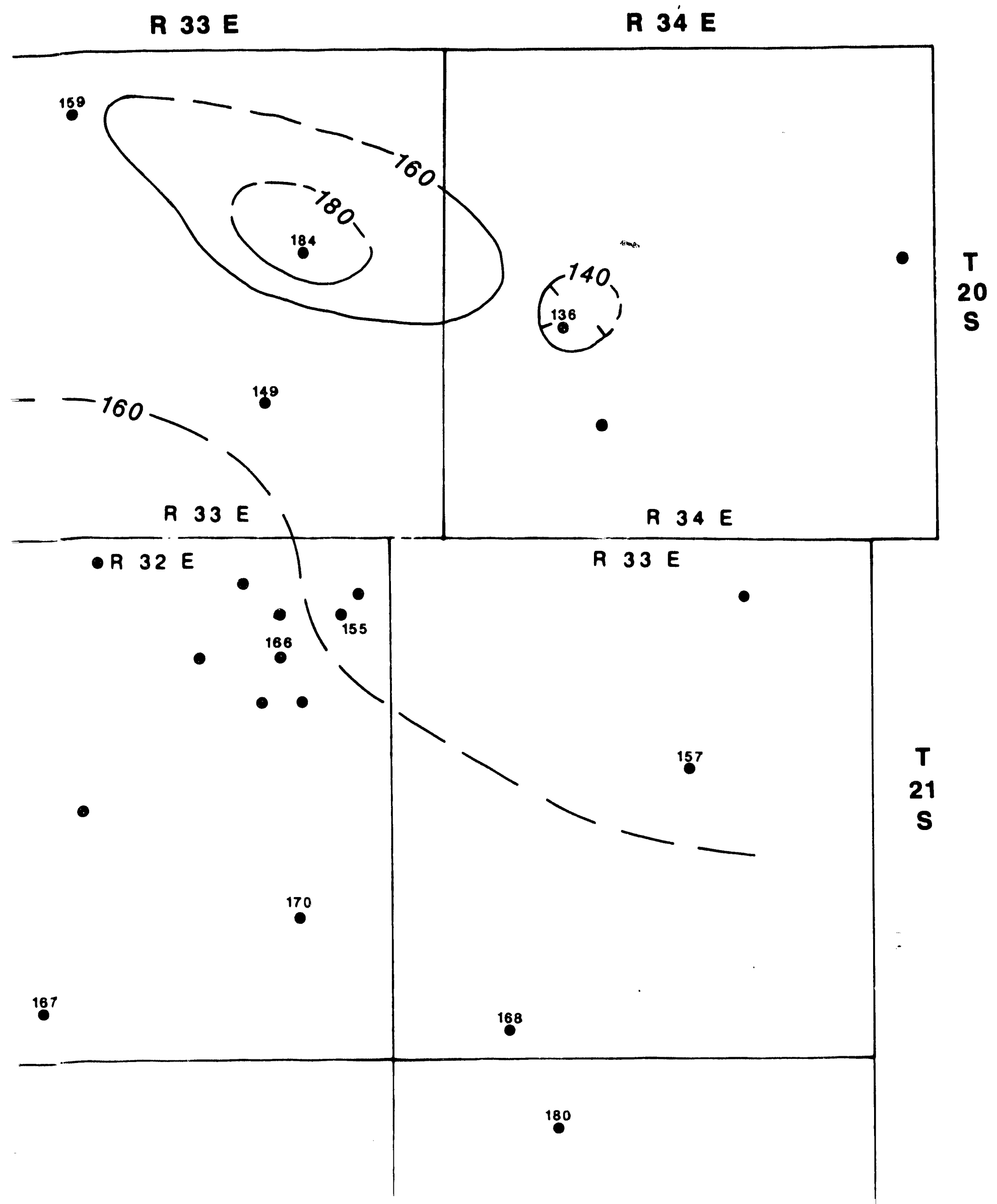




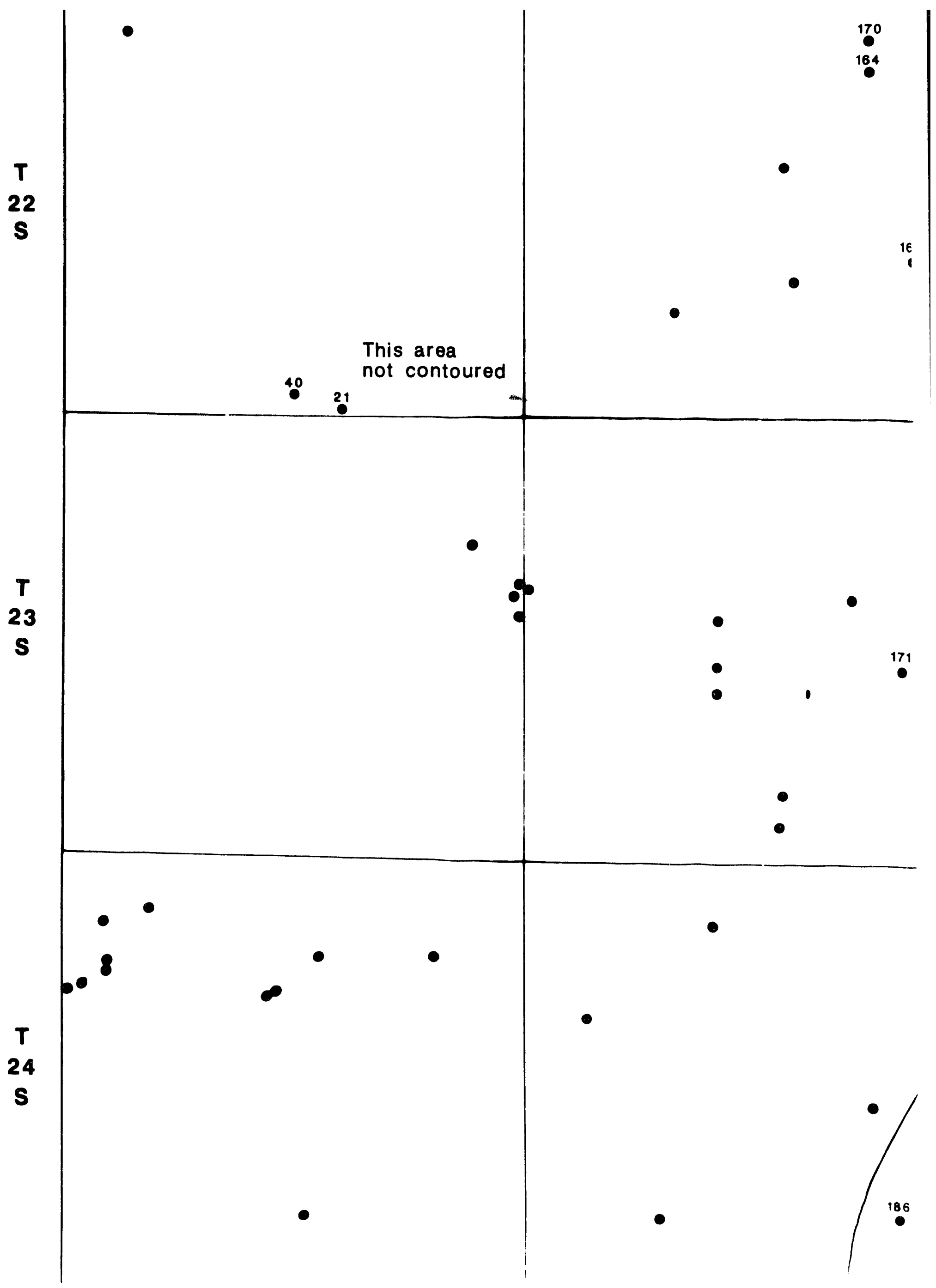




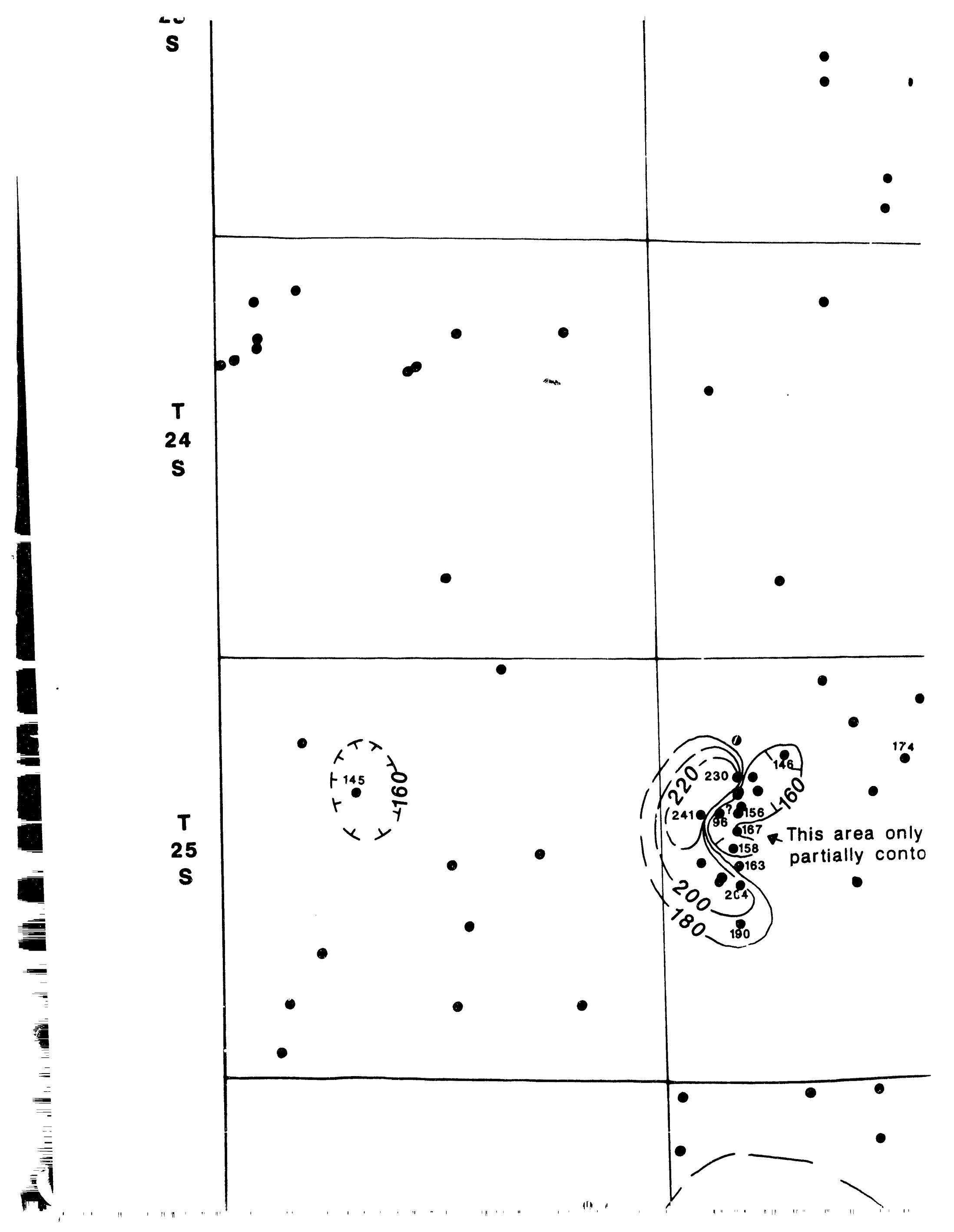






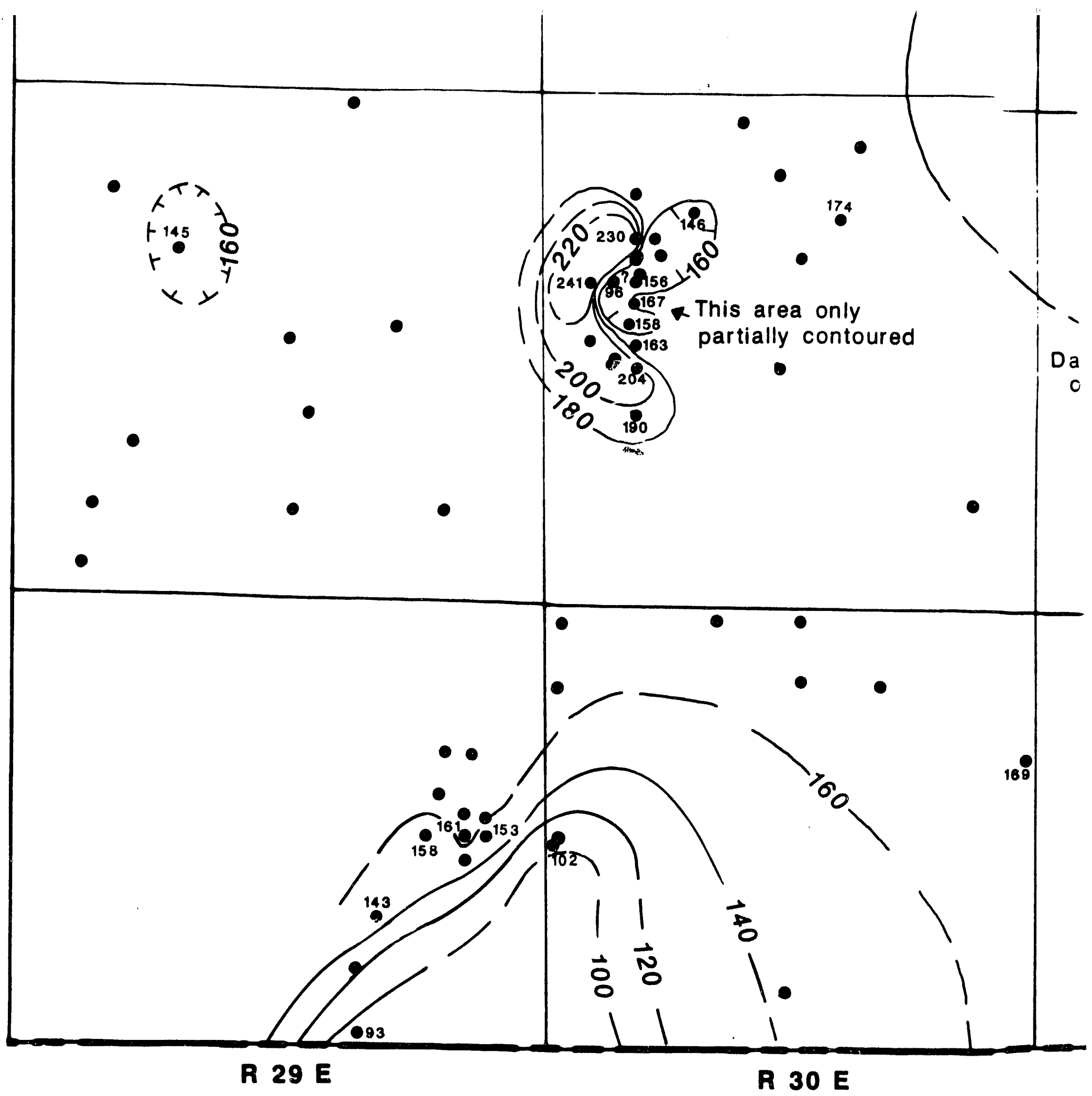

2

0

2 MILES

CONTOUR INTERVAL $=20 \mathrm{FT}$

DASHED CONTOURS REPRESENT

AREAS OF LIMITED STRATIGRAPHIC CONTROL 


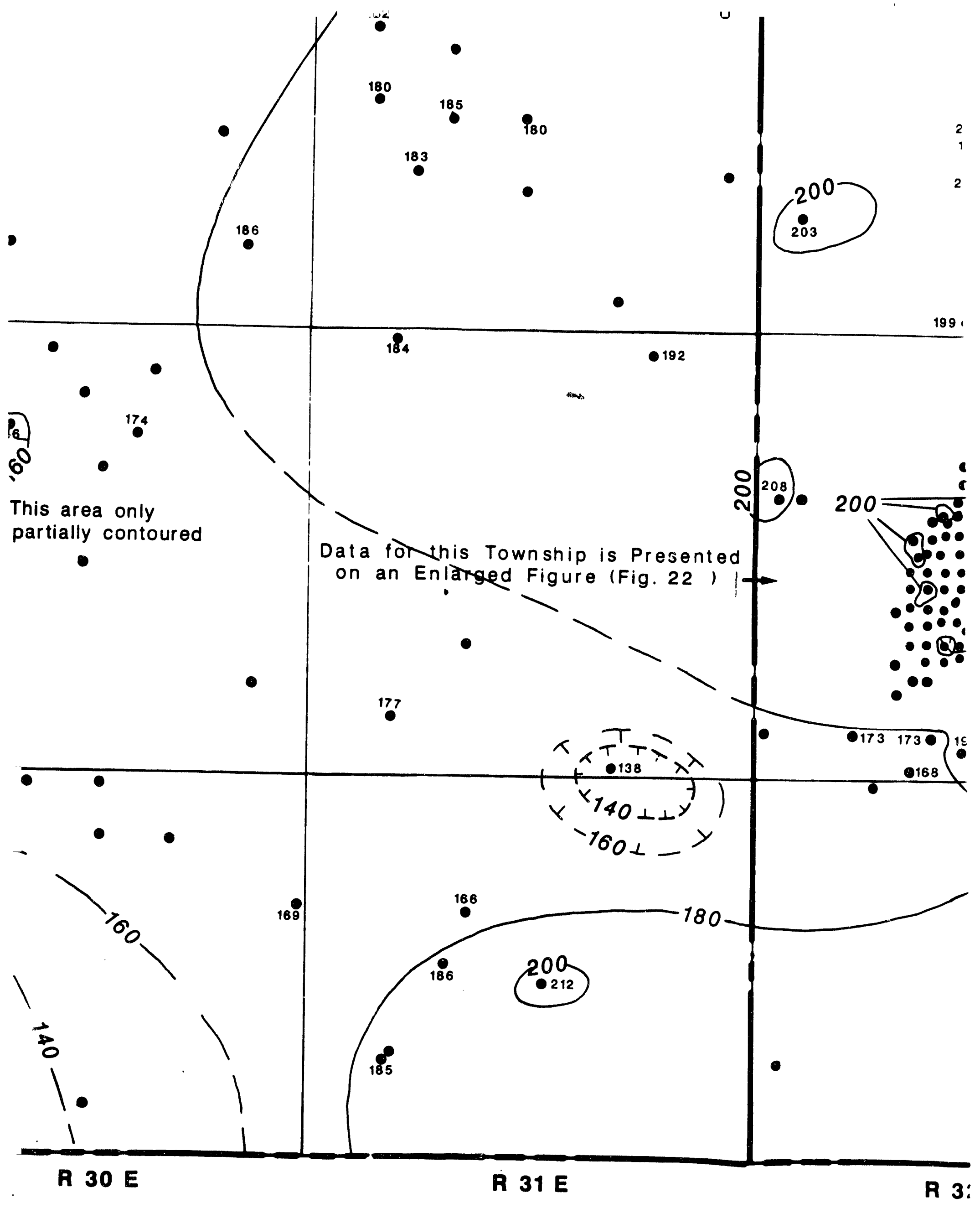




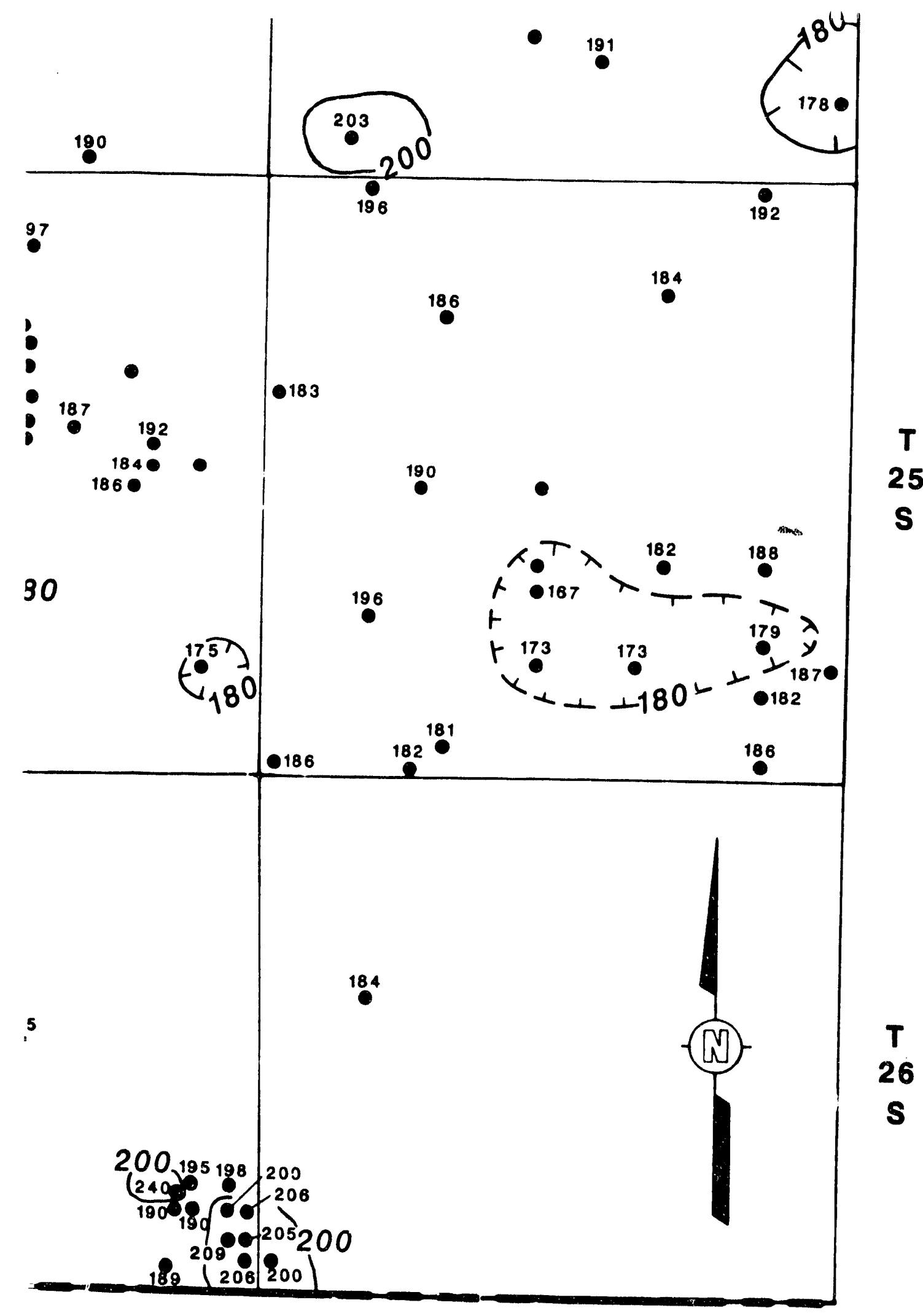

R 33 E

FIGURE 7.2-12

ISOPACH MB109 BASE TO TOP VT

(SEE FIGURE 7.2-8 FOR BOREHOLE IDENTIFIERS! 
Figure 7.1-13

Isopach Salado Top - Base MB103 


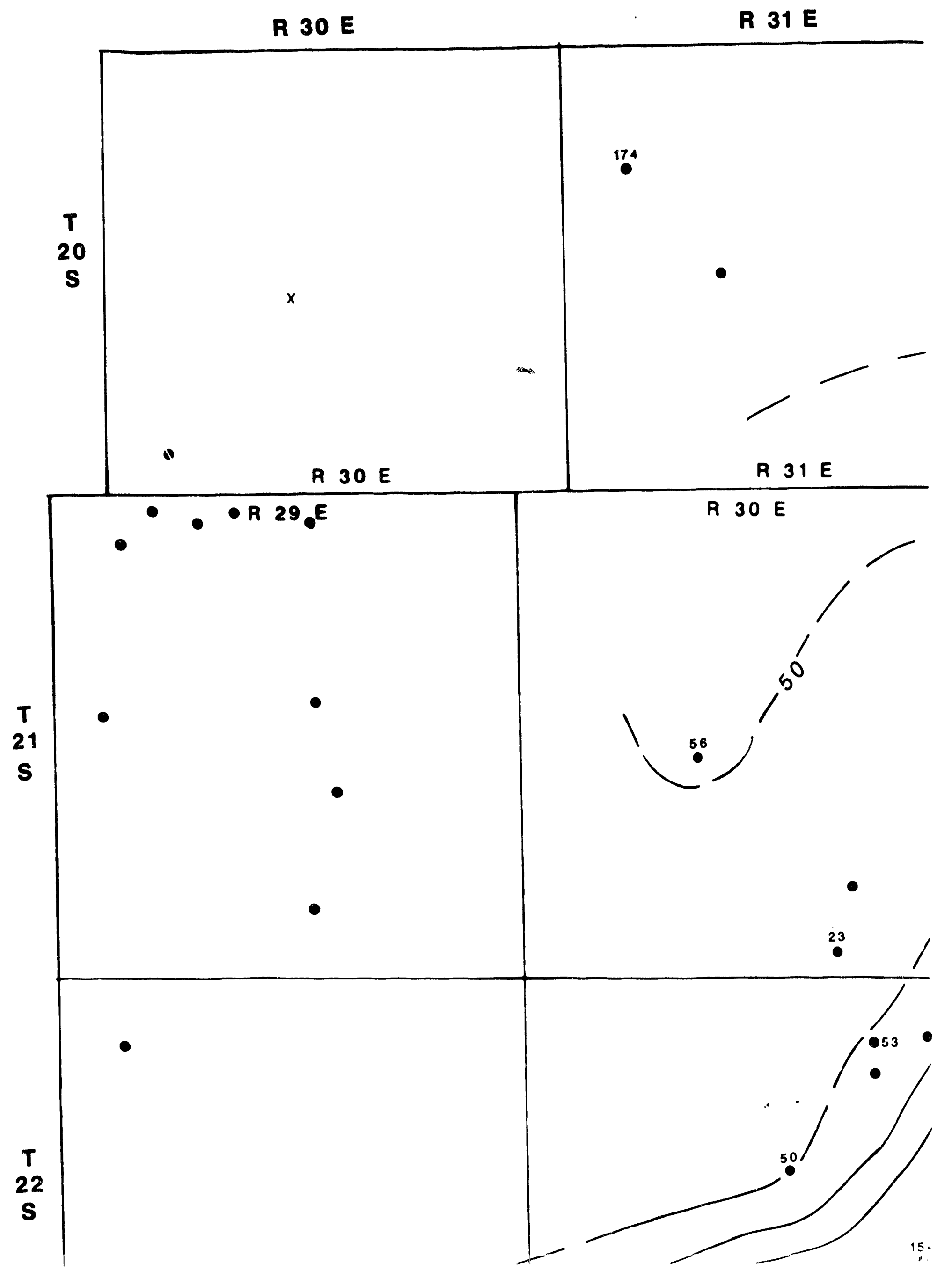


R 32 E

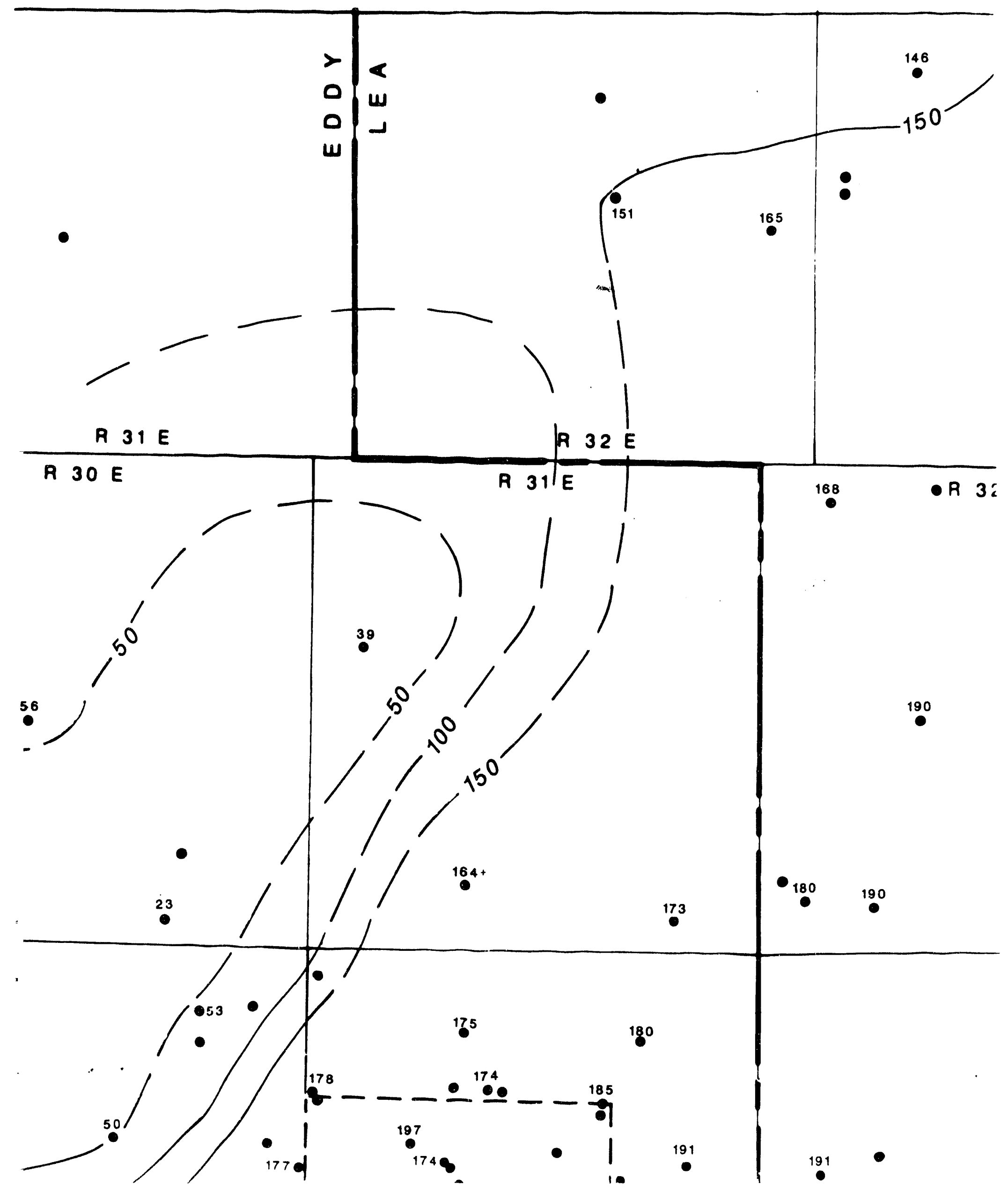




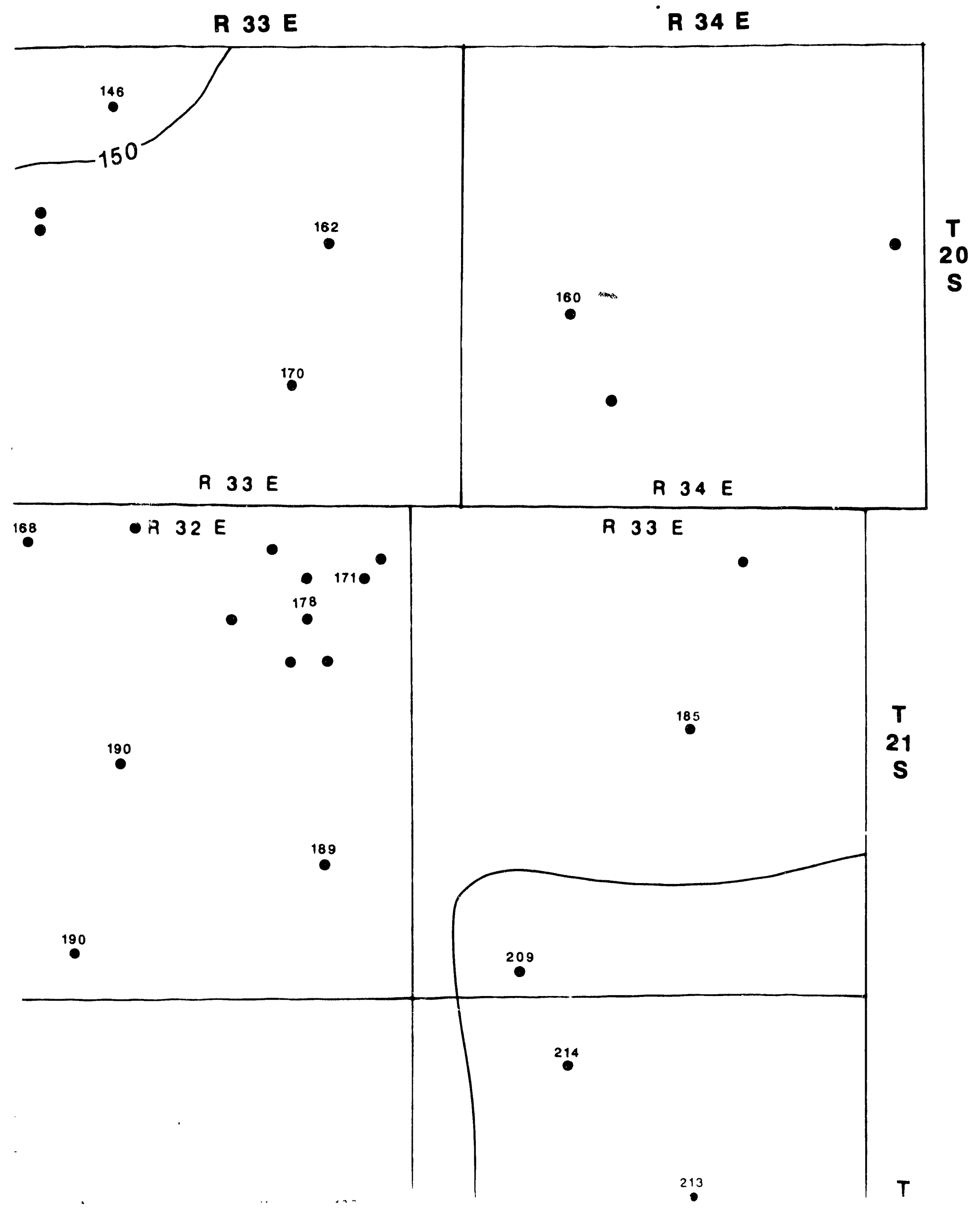




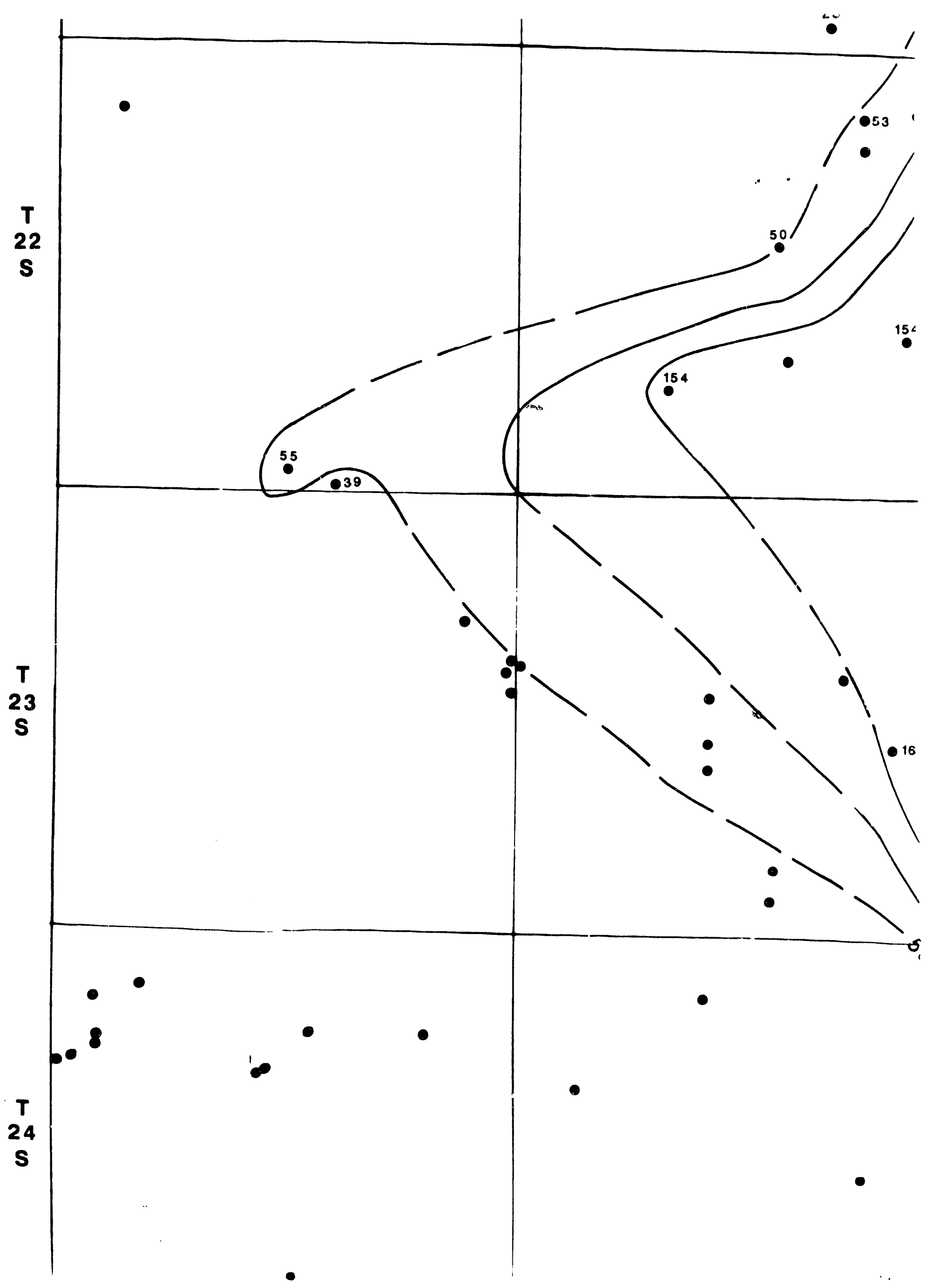




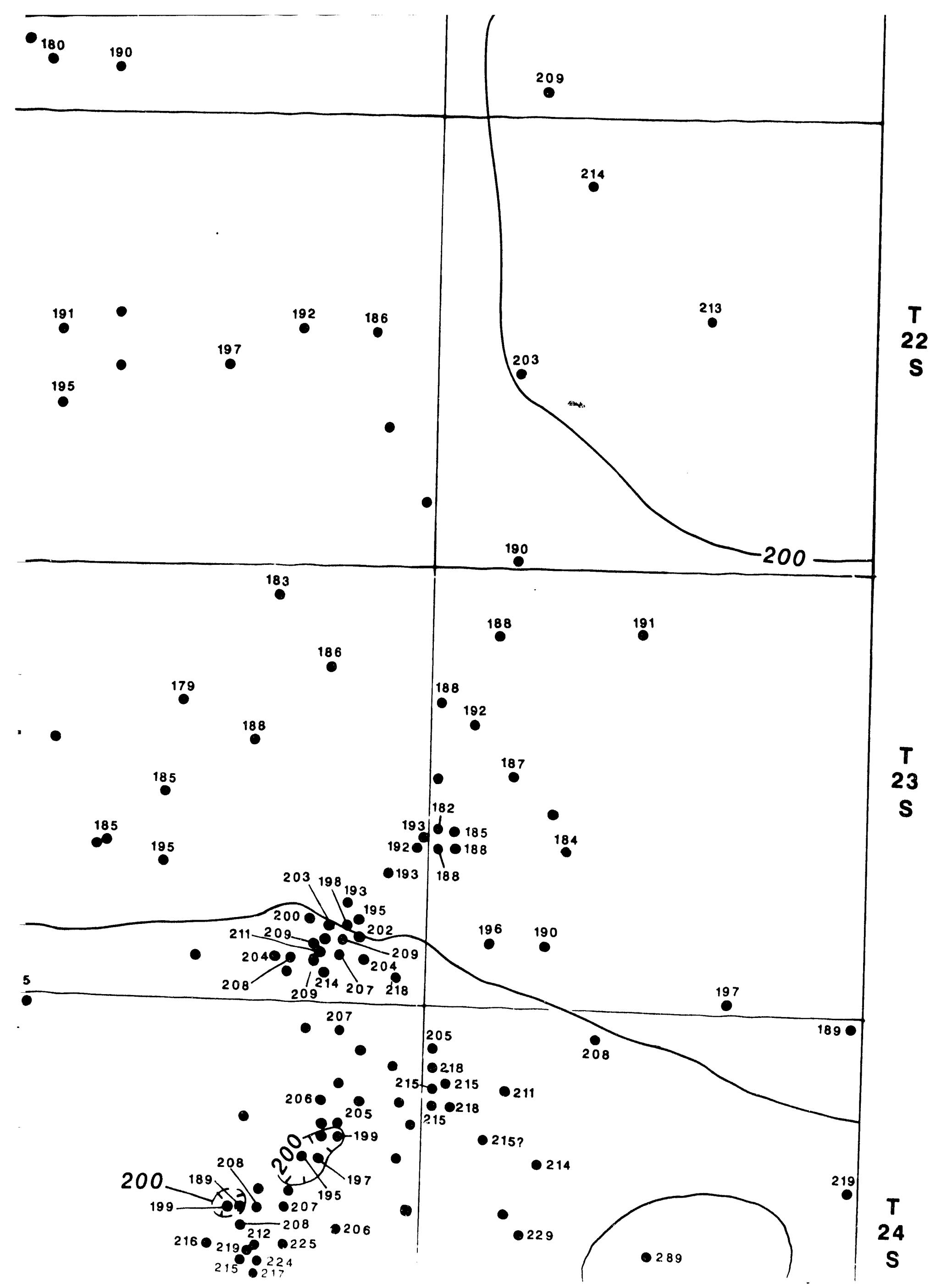




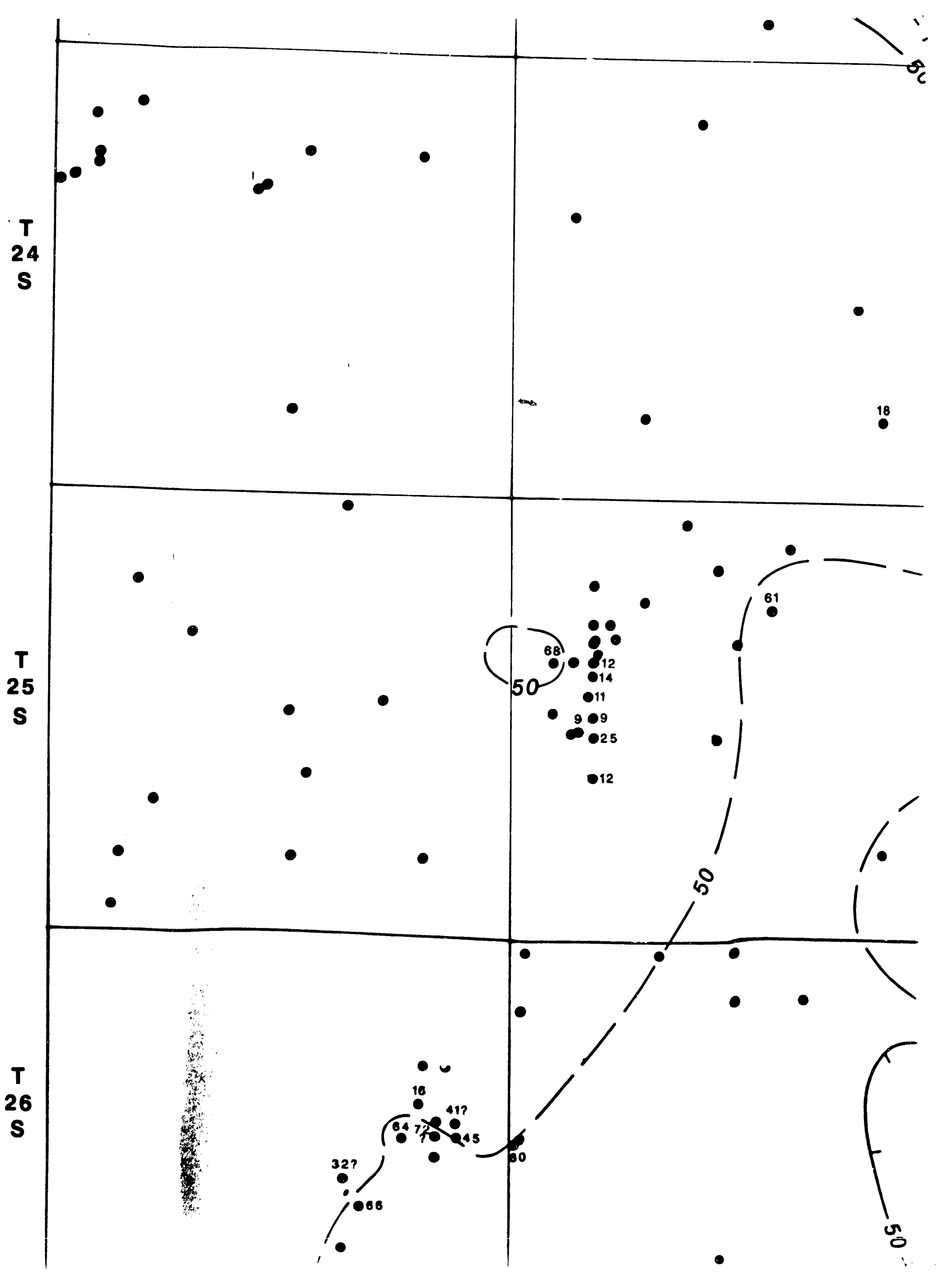




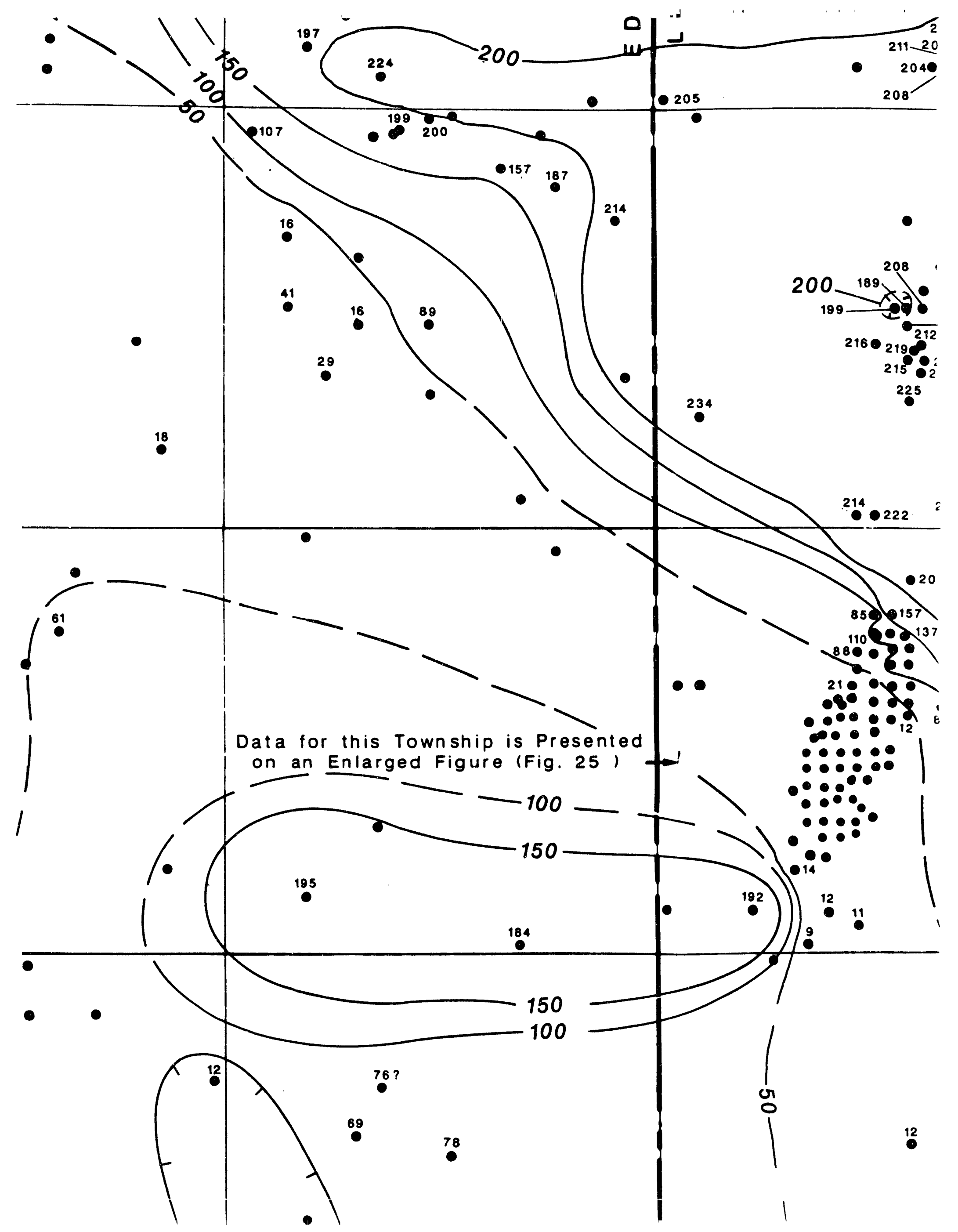




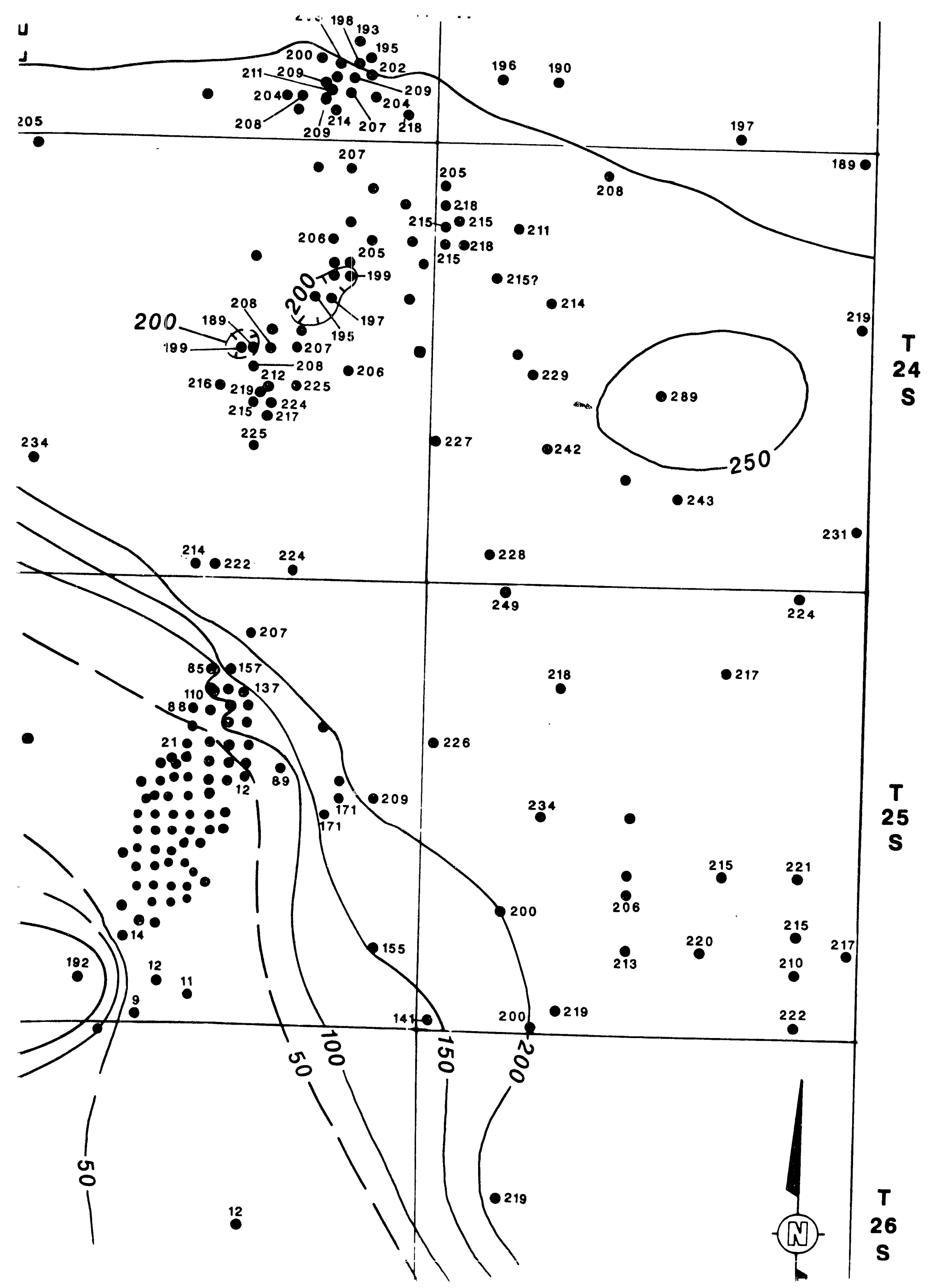




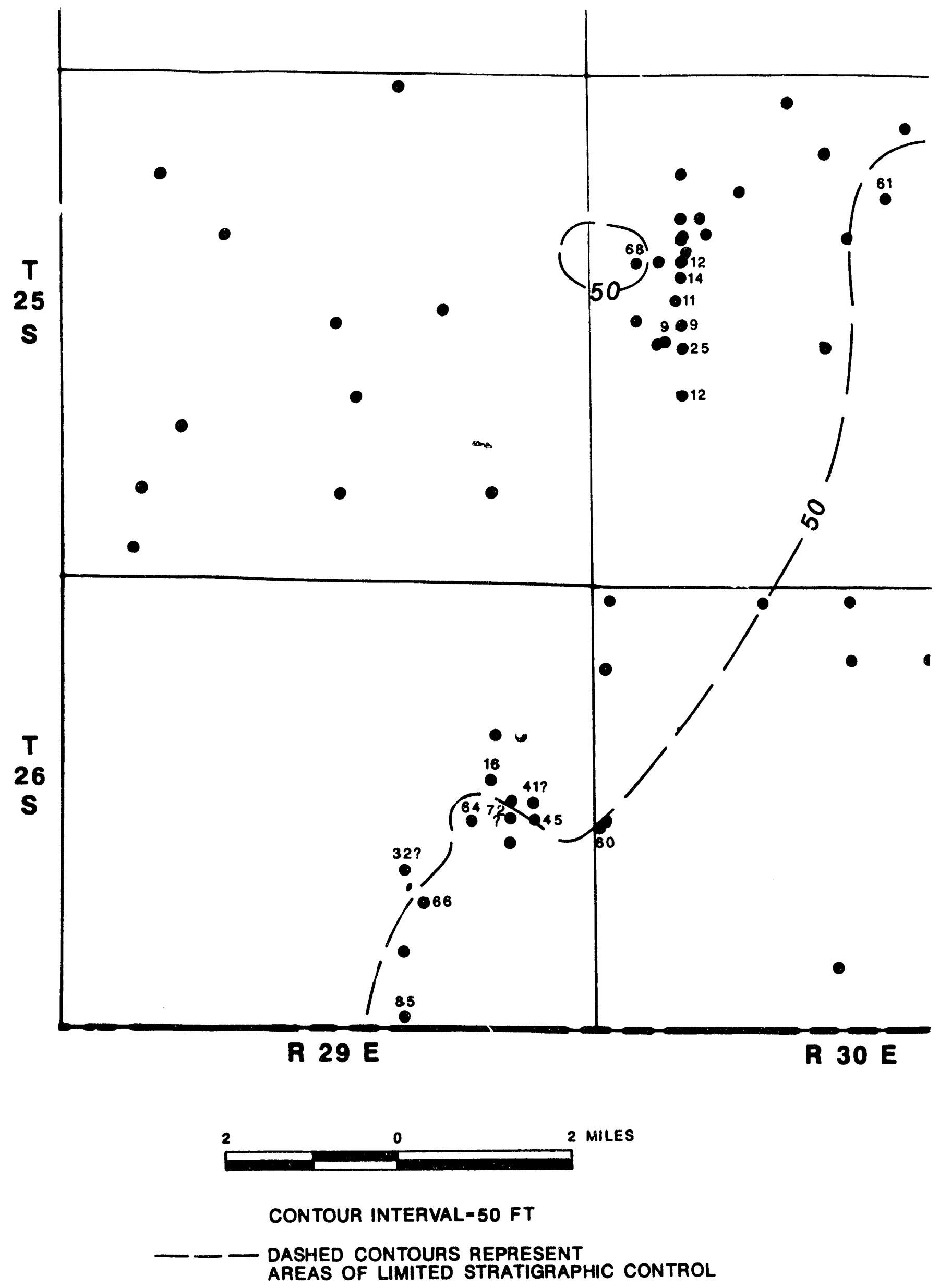




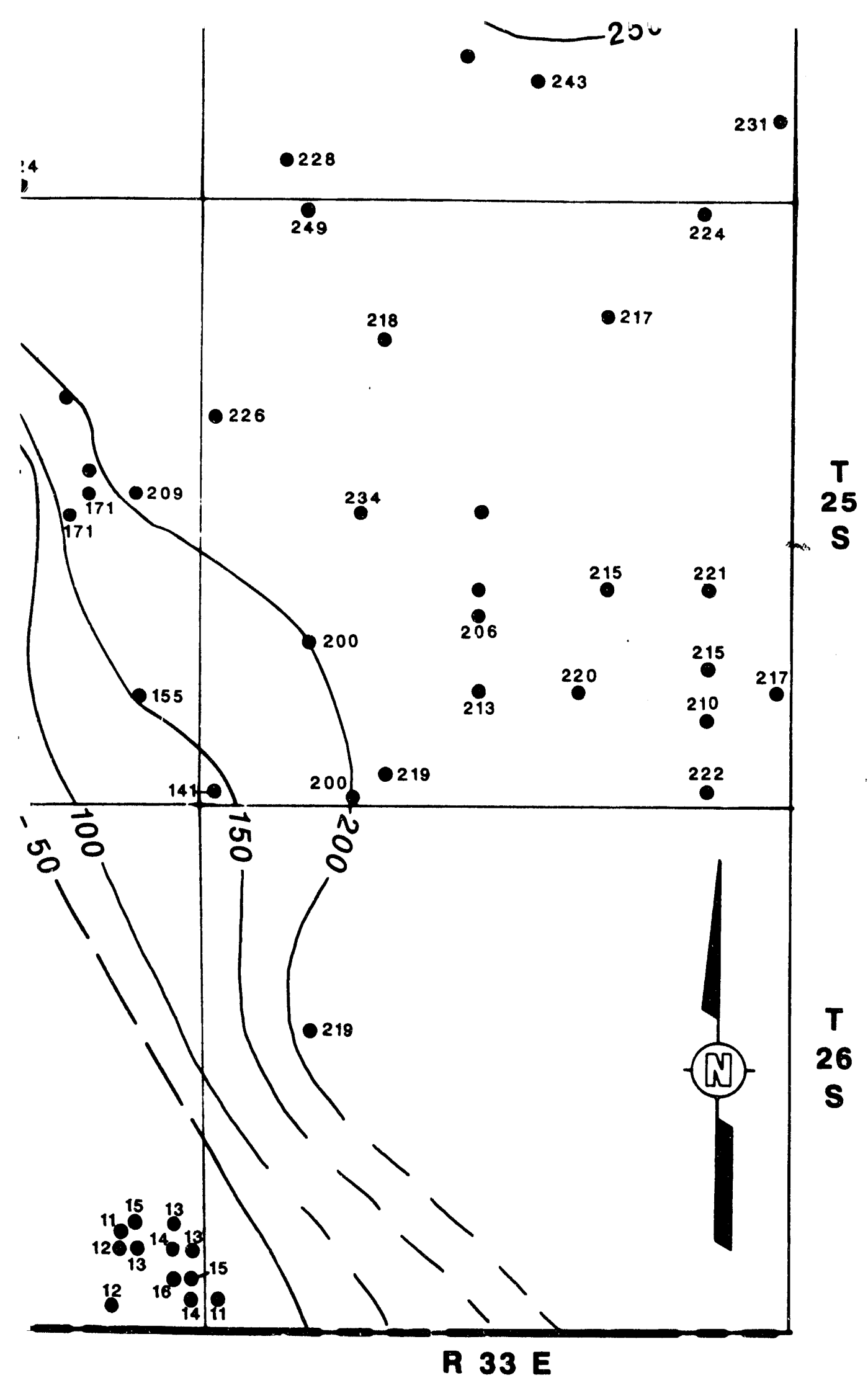


Figure 7.2-14

Structure Contour on Top of Salado 


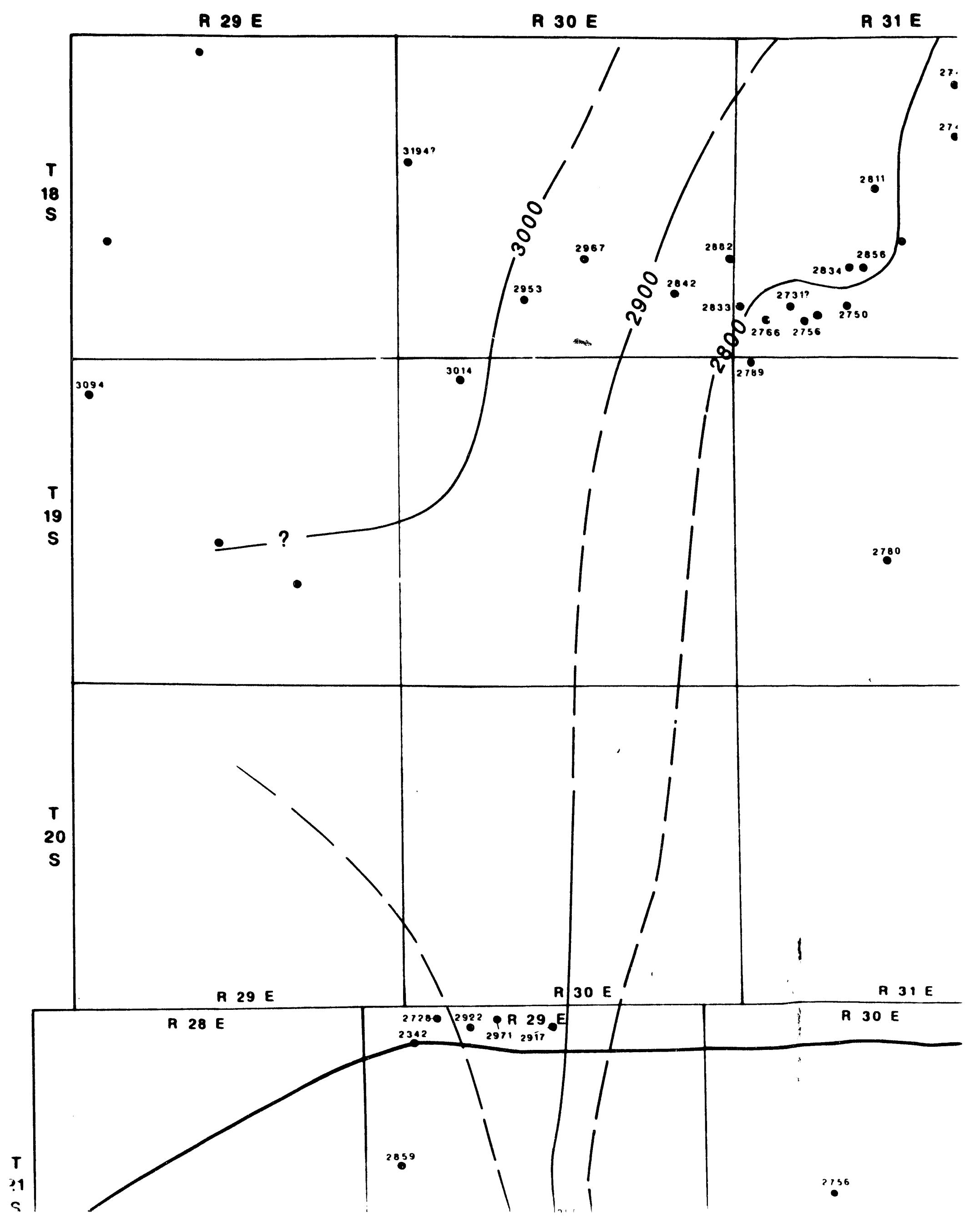




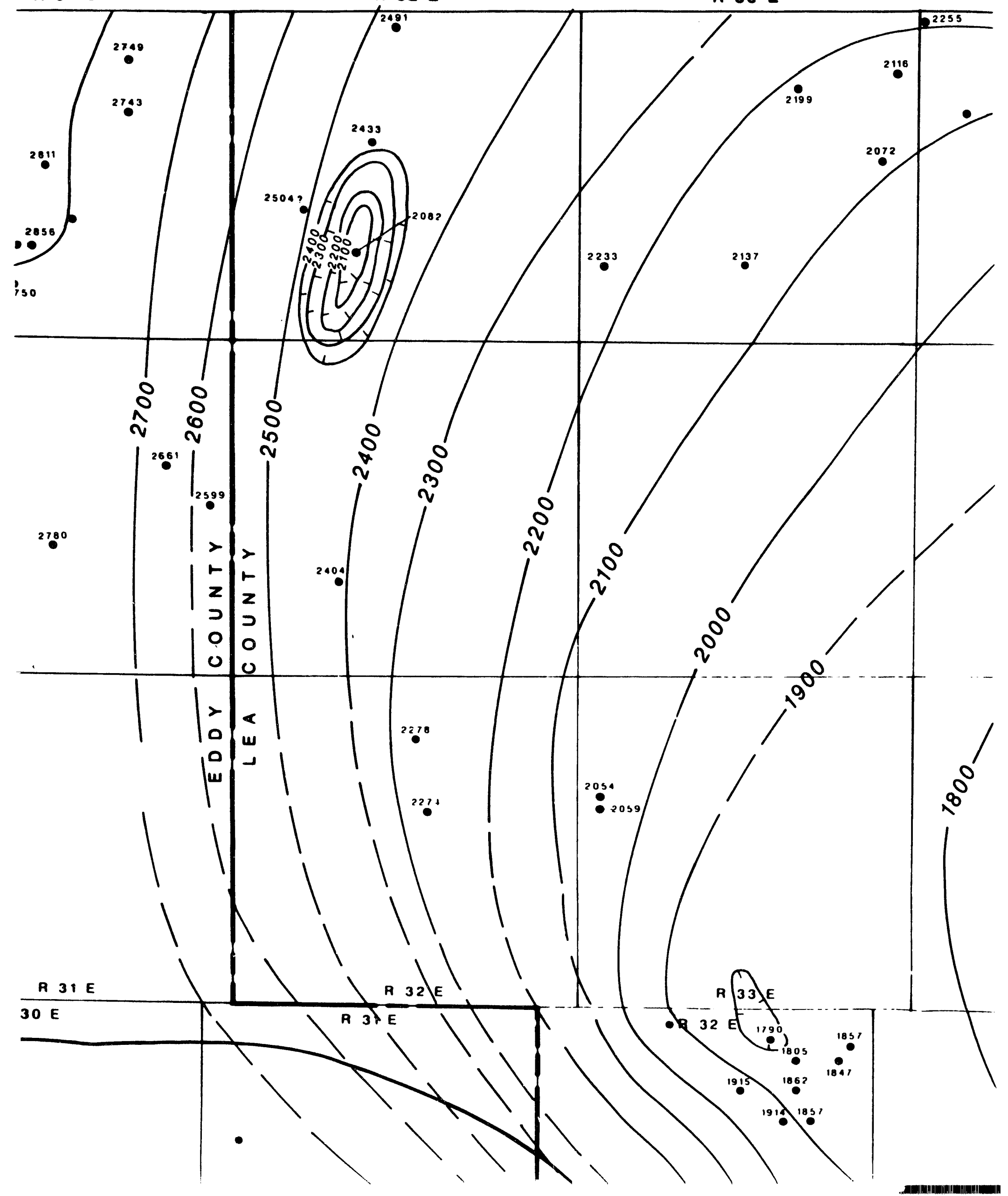




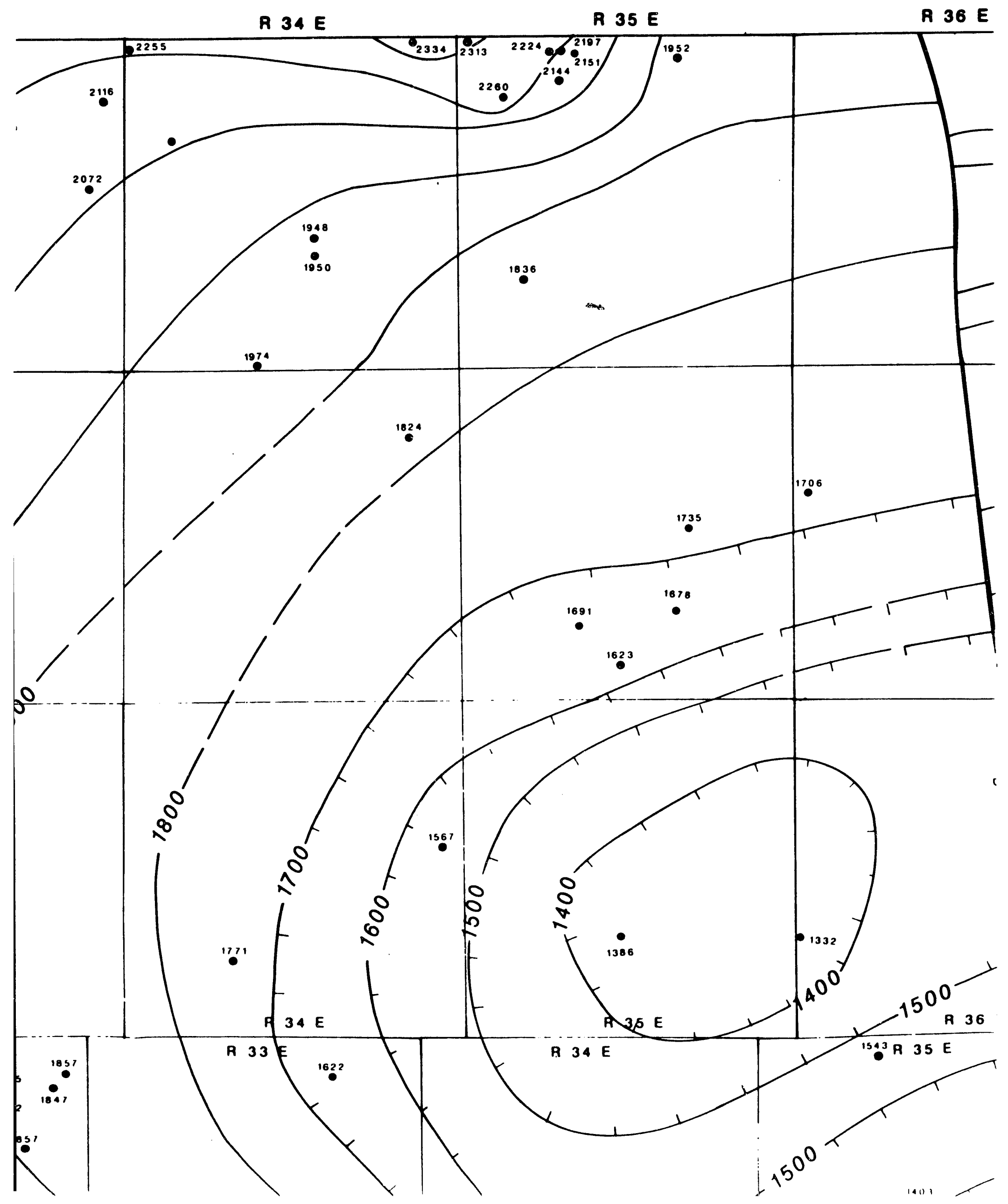




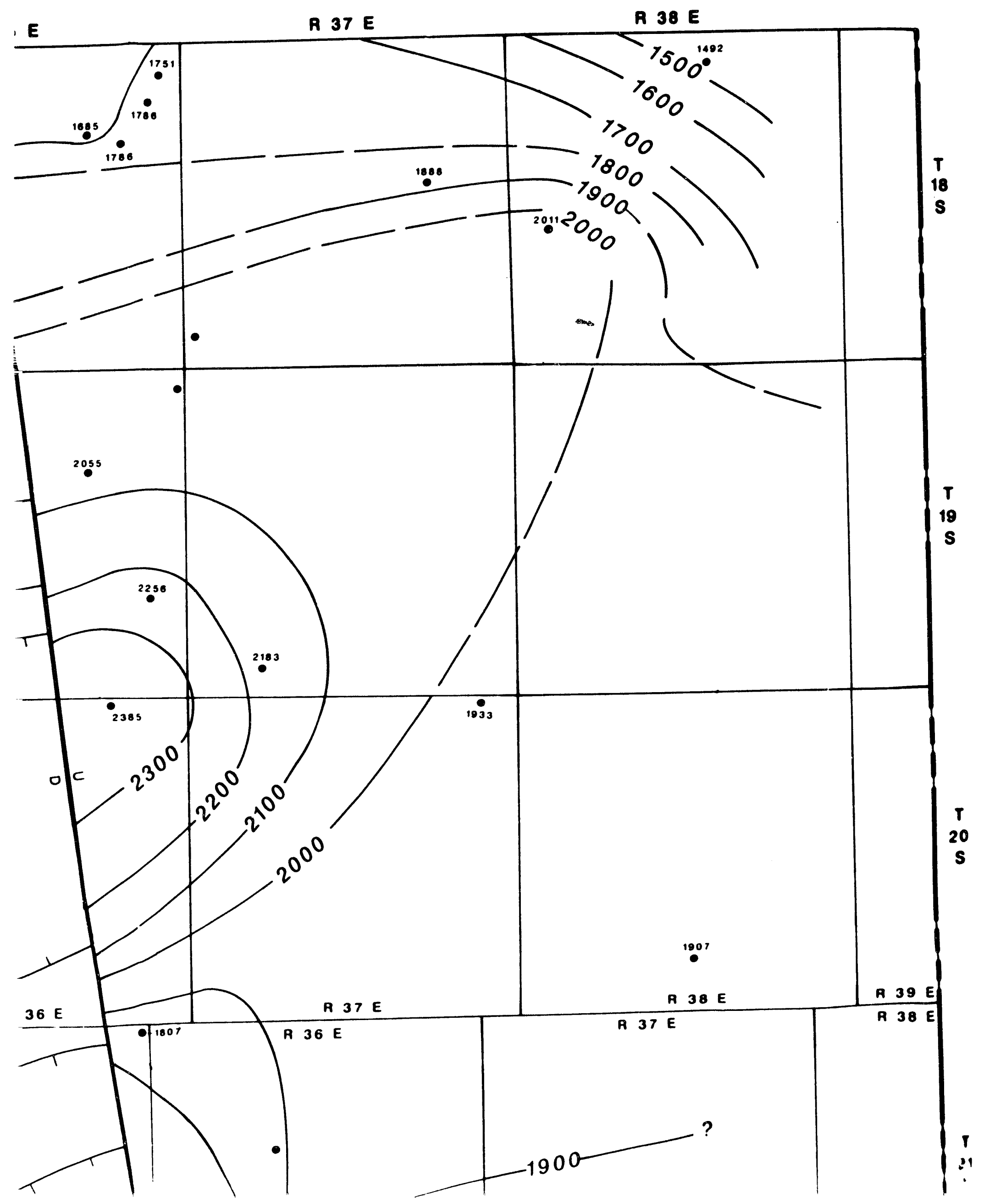




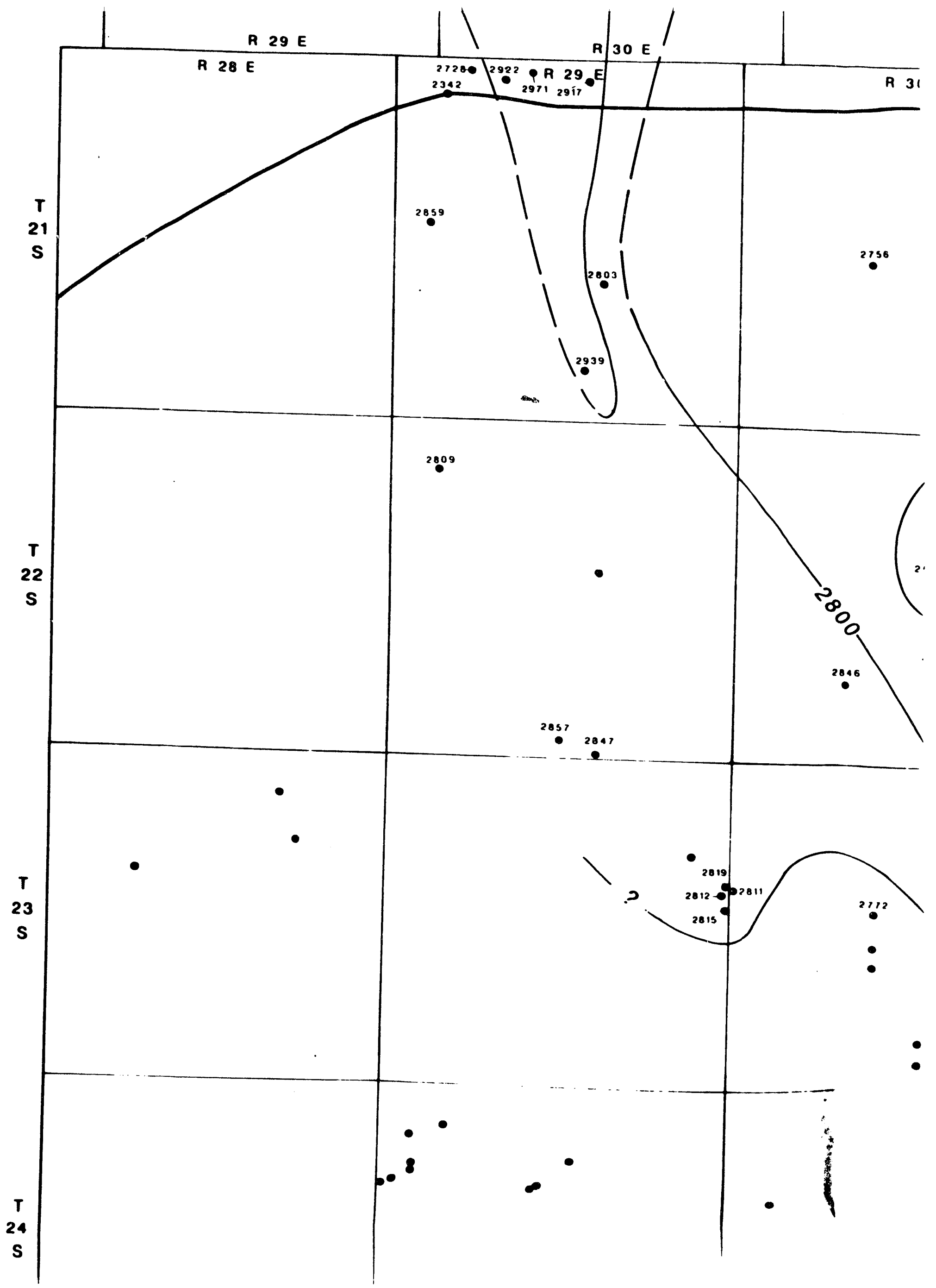




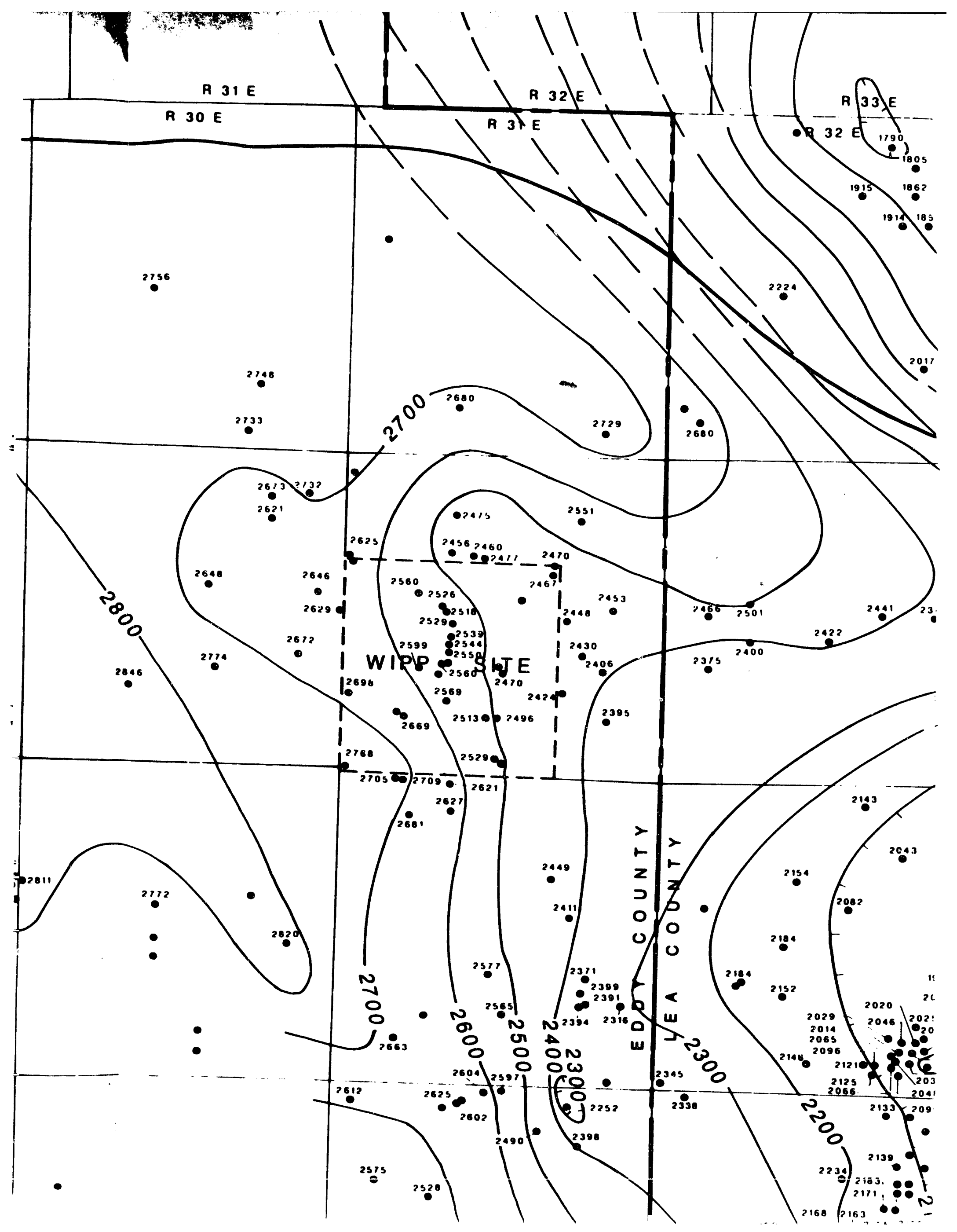




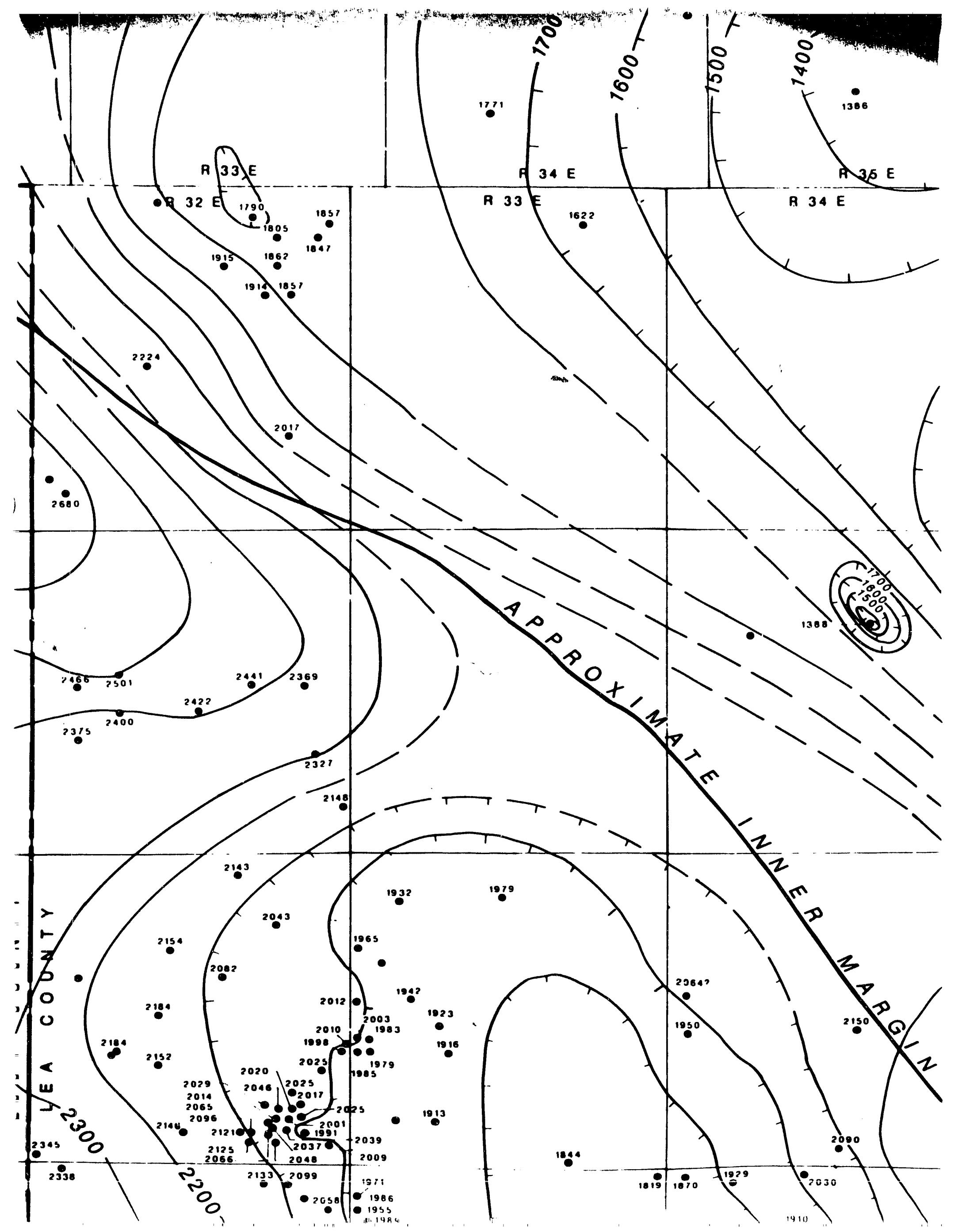




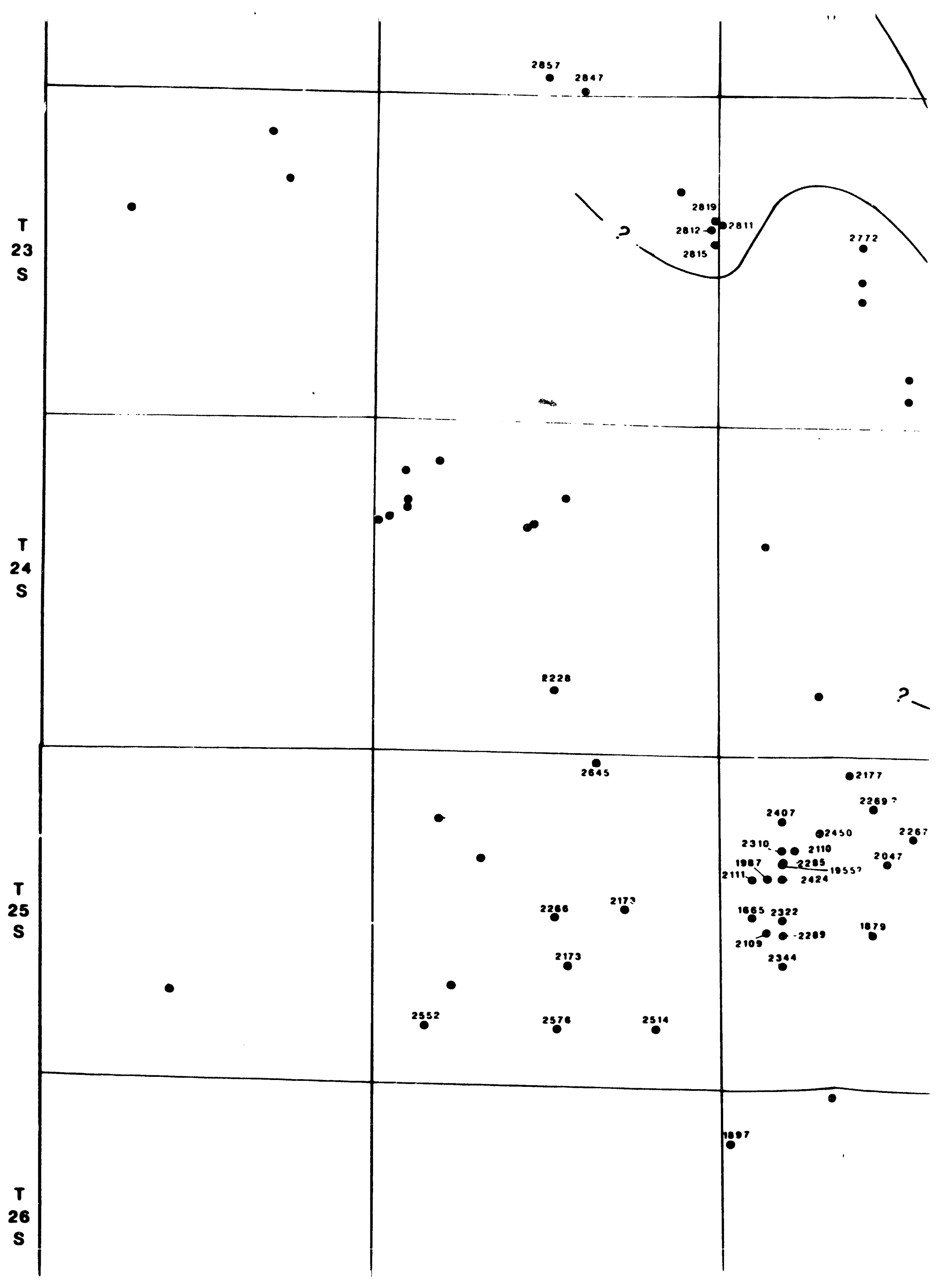




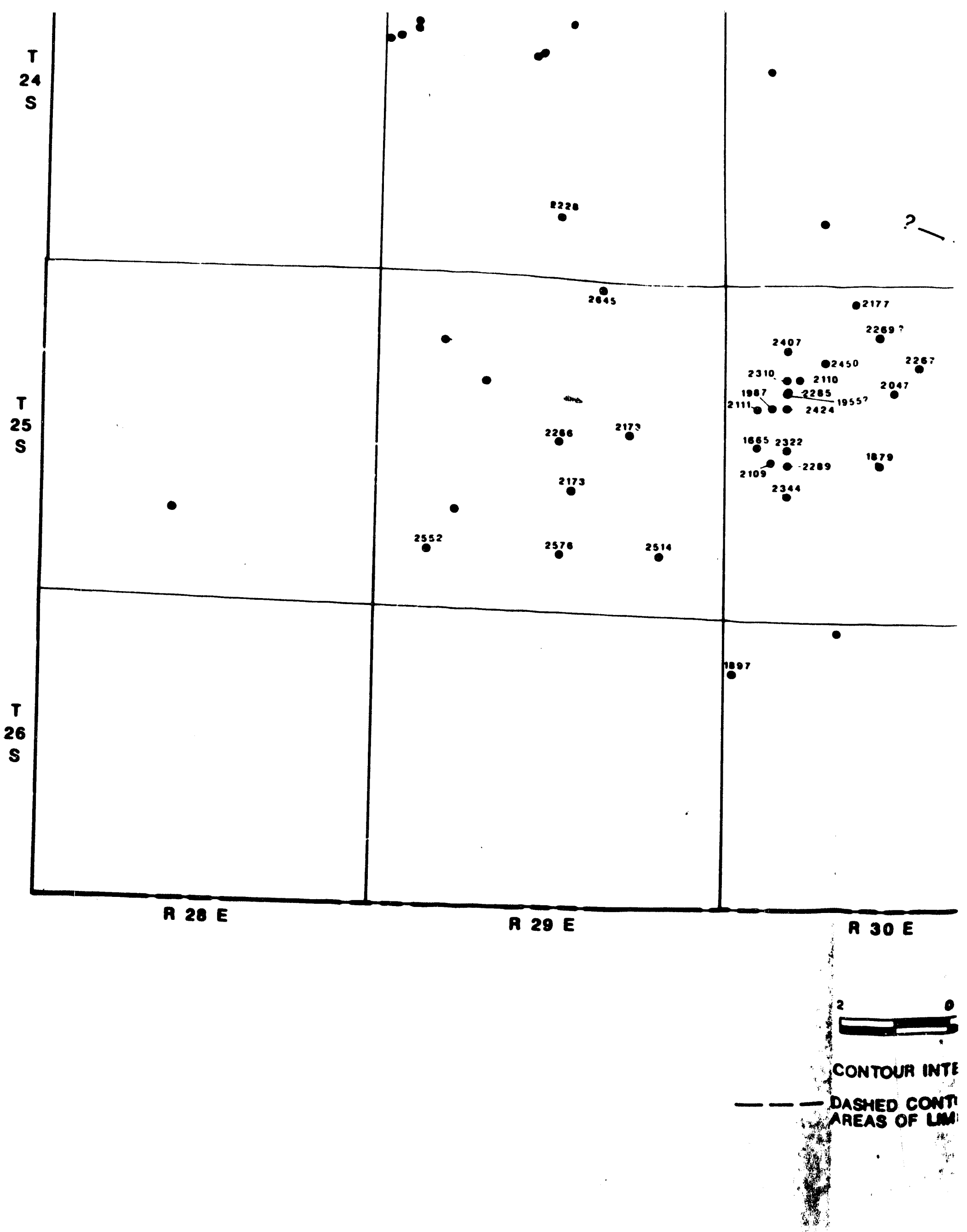

A 28 E

A $29 E$ 


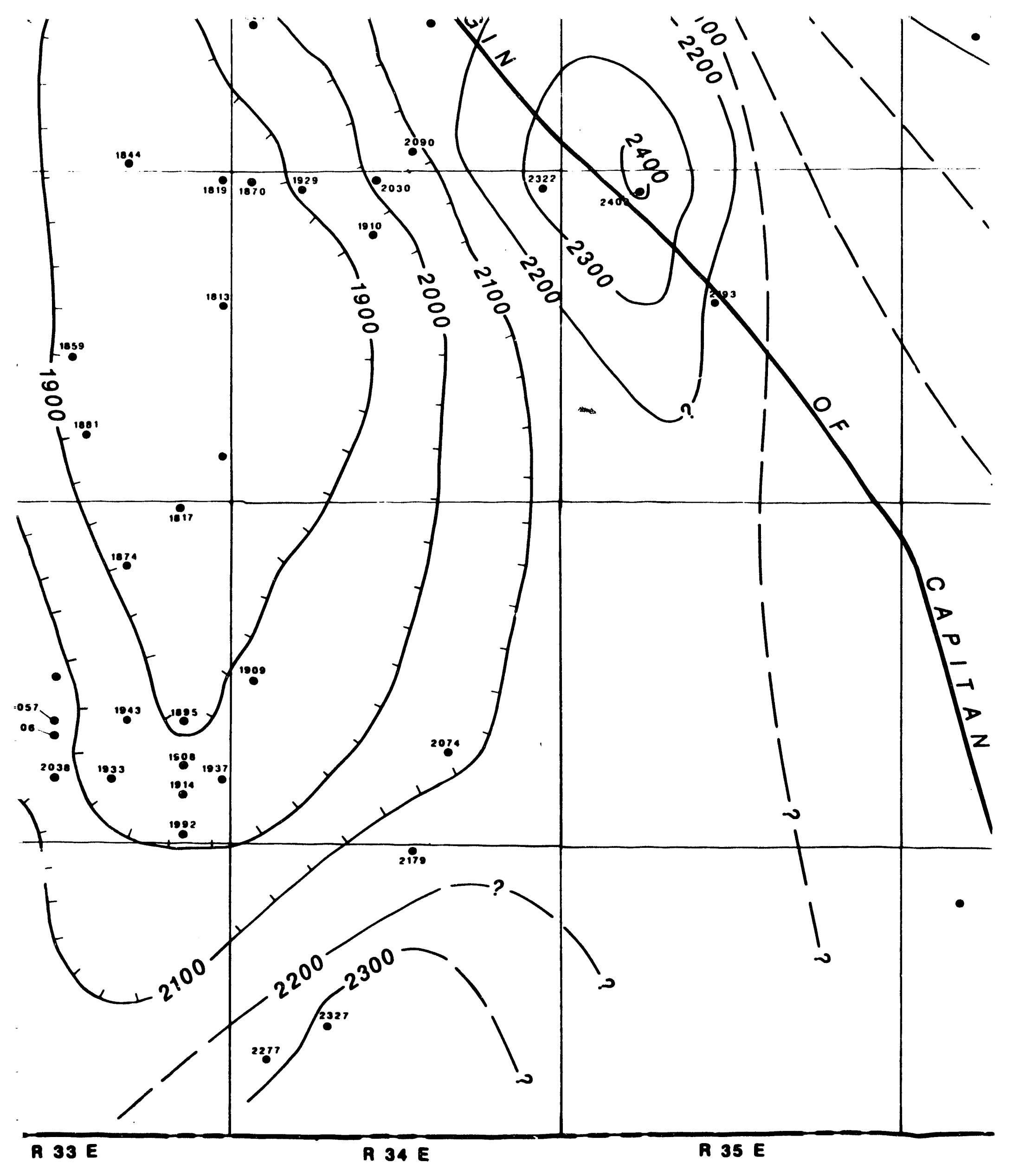



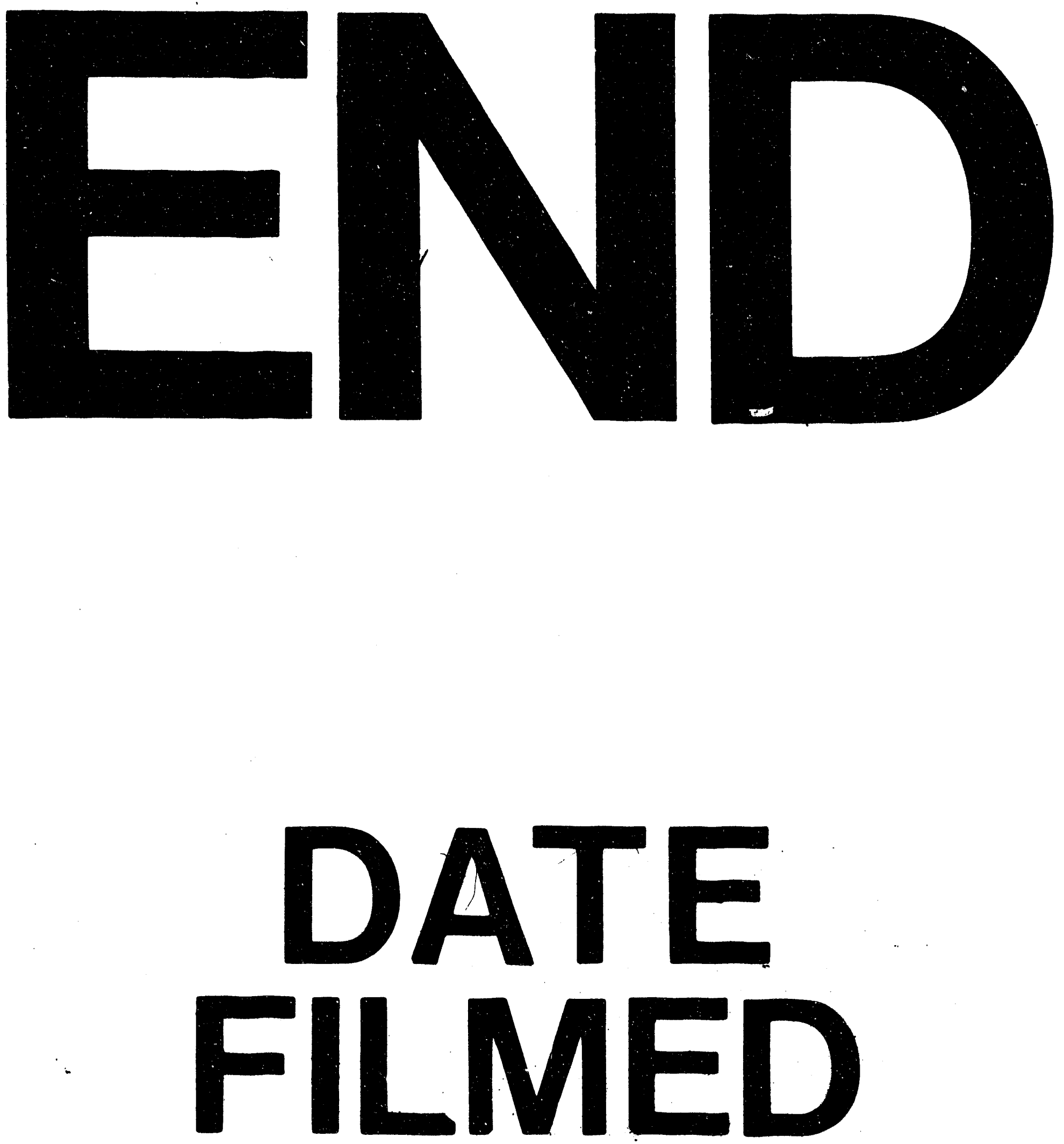

1

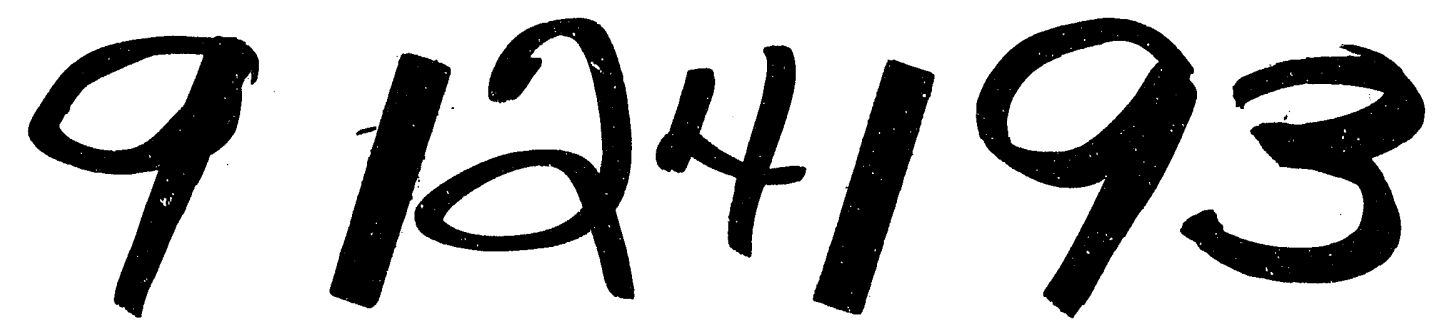


